\title{
Processing face processing
}

Understanding face and facial expression processing in the infant brain

Renata Di Lorenzo

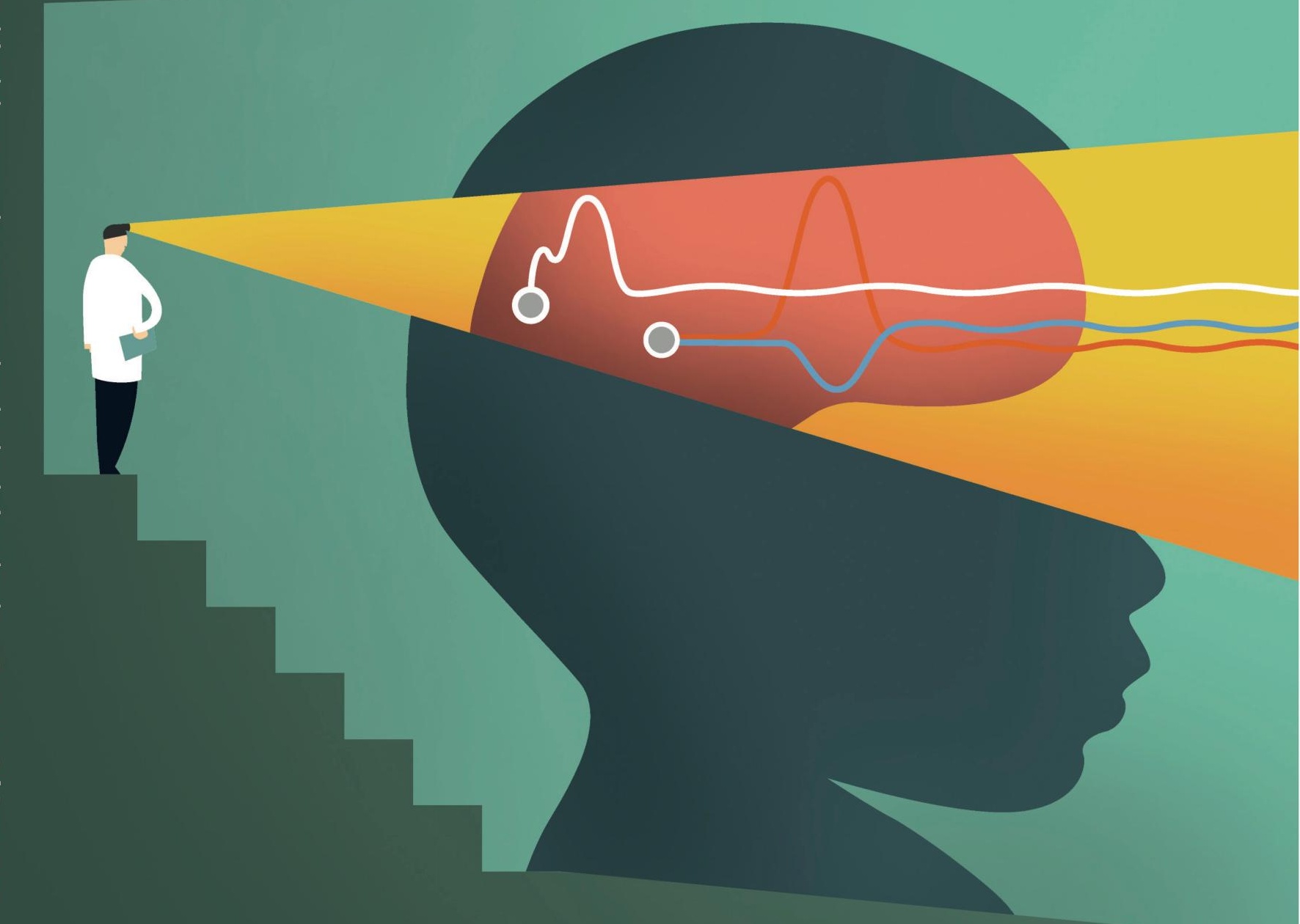




\section{Processing Face Processing: \\ Understanding face and facial expression \\ processing in the infant brain}

Renata Di Lorenzo 
Cover design: (C) Enrico Razzetti

Layout: Renata Di Lorenzo

Printed by: Ridderprint - www.ridderprint.nl

ISBN: 978-90-393-7234-0

Copyright $\odot 2019$ Renata Di Lorenzo

All rights reserved. No part of this thesis may be reproduced in any form by any means without the prior written permission of the author. 


\title{
Processing Face Processing: \\ Understanding face and facial expression processing in the infant brain
}

\begin{abstract}
Analyseren van gezichtsverwerking:
Inzicht in de verwerking van gezichten en gezichtsuitdrukkingen in het babybrein

(met een samenvatting in het Nederlands)
\end{abstract}

\section{Proefschrift}

ter verkrijging van de graad van doctor aan de Universiteit Utrecht op gezag van de rector magnificus, prof.dr. H.R.B.M. Kummeling, ingevolge het besluit van het college voor promoties in het openbaar te verdedigen op

vrijdag 24 januari 2020 des middags te 12.45 uur

door

\section{Renata Di Lorenzo}

geboren op 6 januari 1988

te Tione di Trento, Italië 


\section{Promotor:}

Prof. dr. C. Kemner

\section{Copromotoren:}

Dr. C.M.M. Junge

Dr. C. van den Boomen

Support for the research in this dissertation was primarily provided by the Marie Sklodowska Curie Actions of the European Community's Horizon 2020 Program under grant agreement nº642996 (Braniview). 
To my family 



\section{Contents}

CHAPTER 1 General Introduction

CHAPTER 2 Recommendations for motion correction of infant $\mathrm{ANIRS}$

data applicable to multiple data sets and acquisition systems

CHAPTER 3 Searching for robust infant electrophysiological markers

of face-sensitivity: results from a large longitudinal sample of infants

CHAPTER 4 Brain responses to faces and facial expressions in

5-month-olds: an fNIRS study

CHAPTER 5 Is it fear? Similar brain responses to fearful and neutral faces in infants with a heightened likelihood for Autism Spectrum Disorder

CHAPTER 6 Summary and General Discussion

Appendix I: Nederlandse Samenvatting

Appendix II: Acknowledgements

Appendix III: List of Publications

Appendix IV: About the Author 

Chapter 1

General Introduction 


\section{Introduction}

I think of children as little scientists: they explore and learn about the world by testing and touching everything that grabs their attention. Imagine Laura, a 7-month-old girl playing with her toys on the floor. Suddenly, she notices the electricity socket on the wall in front of her. She immediately leaves all her toys and starts crawling towards the socket. If nothing happens, she might go ahead and touch the socket, but if her mother looks at her with a very fearful expression Laura will probably stop and change direction. Being able to discriminate faces from other objects and understand emotional signals from others' faces is crucial to achieve successful social communication for humans of all ages, but especially for pre-verbal infants as Laura.

Important changes in behavioural responses to faces and emotional facial expressions occur during the first year of post-natal life. For instance, infants between six-nine months of age can categorize faces based on gender, race, and attractiveness and between five-seven months demonstrate a bias to attend fearful facial expressions (for reviews see Anzures, Quinn, Pascalis, Slater, \& Lee, 2010; Leppänen \& Nelson, 2009). A number of studies is investigating the neural activity underlying face and facial expression processing across infancy as well as the factors that could affect its development. Knowledge on how the infant brain typically processes faces within the first year of life is crucial to fully understand why and how behavioural changes in the responses to faces arise, which is also relevant for studies on social communication development. Furthermore, it provides a baseline to understand developmental disorders where social communication is atypical, such as in Autism Spectrum Disorder (ASD).

In this introductory chapter, I am first going to examine the main strengths and limitations of the techniques typically used to measure brain responses to faces in infancy. Next, I will review what is currently known about the brain networks underlying face and facial expression processing in adulthood and infancy. Finally, I will provide an outline of this dissertation. 


\section{Methods to study the development of face and facial expression processing in the infant brain}

To uncover the development of face and facial expression processing in the infant brain, two main techniques are typically used: functional near-infrared spectroscopy (fNIRS) and event-related potentials (ERPs). Developmental researchers often adopt these techniques to contrast brain responses to two or more experimental conditions (e.g., pictures of faces vs. other objects; happy vs. fearful faces), which gives an indication of whether the two conditions are processed in similar or different ways. Both techniques are relatively child-friendly and do not require the active response of the child, which is of fundamental importance in infant studies. ANIRS and ERPs have different resolution properties: fNIRS has a relatively higher spatial resolution, while ERPs have a higher temporal resolution. These properties allow to tackle different questions related to brain activation. Specifically, whereas with fNIRS it is possible to test which cortical areas respond to faces or emotional facial expressions, with ERPs ${ }^{1}$ one can assess when the processing of faces or emotions within faces takes place in the brain. Although these techniques have great potential in unravelling the development of infant face and facial expression processing, they present also several challenges. In the following paragraphs, I will describe principles, interpretations of the output measures, and current difficulties specific of each method.

\section{functional Near-Infrared Spectroscopy - fNIRS}

fNIRS is a neuroimaging technique that infers localized brain activation by measuring changes in concentrations of oxygenated- $(\mathrm{HbO})$ and deoxygenated-haemoglobin (HbR). Typically, neural activation in response to a stimulus triggers an increase in blood flow and oxygen delivery to the active region. Crucially, the oxygen delivered exceeds the demands of the brain tissue which results in a temporary increase of $\mathrm{HbO}$

\footnotetext{
${ }^{1}$ Note that ERPs are collected via electrophysiological data acquisition systems which also allow for brain source-localization. Source-localization is most effective when brain activity is sampled from more than 32 electrodes (DeBoer, Scott, \& Nelson, 2007). Given that the studies in the current thesis used 32 electrode acquisition systems I limited my analyses to ERPs and did not include source-localization.
} 
coupled with a (smaller) decrease of $\mathrm{HbR}$ concentration in the activated area (Villringer \& Chance, 1997). This metabolic response, known as haemodynamic response function (HRF; see Figure 1.1), peaks several seconds after neural activation (for a comparison of adult and infant HRF see Arichi et al., 2012). To estimate changes in $\mathrm{HbO}$ and $\mathrm{HbR}$ concentrations, fNIRS exploits the optical properties of human biological tissue in the near-infrared range (optical window $\sim 650-950 \mathrm{~nm}$; Scholkmann et al., 2014). In particular, near-infrared light can travel through head tissues (i.e. skin, scalp, skull, adipose tissue) and is absorbed by $\mathrm{HbO}$ and HbR (Jacques, 2013; Villringer \& Chance, 1997), which are characterized by separated absorption coefficients that vary with wavelength. Most fNIRS systems use two wavelengths that are selectively sensitive to one haemoglobin type (e.g., one < $780 \mathrm{~nm}$ and one $>830 \mathrm{~nm}$; Scholkmann et al., 2014) to quantify changes in $\mathrm{HbO}$ and $\mathrm{HbR}$ concentrations. Although note that both wavelengths are used to measure change in concentrations of $\mathrm{HbO}$ as well as $\mathrm{HbR}$. By taking advantage of these properties, fNIRS measures the change in light intensity migrating from one source that projects light from the scalp into the cortex to a scalpdetector. When near-infrared light is sent into the brain, only $20 \%$ is absorbed by the brain tissue, while the remaining $80 \%$ is scattered (Elwell, 1995). Scalp detectors record only a portion of the scattered light, which mainly follows a banana-shaped trajectory from the source. The intensity of the detected light is converted into $\mathrm{HbO}$ and $\mathrm{HbR}$ concentrations using the modified Beer-Lambert law (Arridge, Cope, \& Delpy, 1992; Delpy et al., 1988). The modified Beer-Lambert law computes such conversion by taking into account the pathlength ${ }^{2}$ and media through which the light travels, including the scattering loss. Source-detector pairings are commonly called channels. The depth at which each channel samples into the cortex is determined by the source-detector distance on the scalp, which in infant research is $2-3 \mathrm{~cm}$, corresponding to a depth of measurement of $\sim 1.2-1.5 \mathrm{~cm}$ from the scalp (for a review see Lloyd-Fox, Blasi, \& Elwell,

\footnotetext{
${ }^{2}$ The pathlength is the additional distance the light travels from source to detector due to scattering. If there was no scattering the light would travel in a straight line. Because of scattering, the light travels a winding path, making the total distance travelled by the light larger. Pathlength also changes with age.
} 
2010; see also Lloyd-Fox et al., 2014). Hence, with fNIRS we can infer only cortical but not subcortical activity.

There are several experimental paradigms that can be employed to measure haemodynamic changes relative to a specific stimulation (for an overview of infant paradigms see Lloyd-Fox et al., 2010). For infant research, block design is the favourite one: infants are presented with an experimental condition for a period of 5-30 s alternating with a baseline condition. The baseline condition needs to be sufficiently long to allow the haemodynamic response started in the experimental condition to return to a baseline level before a new stimulus is presented. Compared to the experimental condition, the baseline condition is typically designed to induce no activation in the region of interest or at least a lower activation than the one elicited by the experimental condition (Lloyd-Fox et al., 2010). Hence, the haemodynamic response elicited by the experimental condition is assessed relative to the baseline condition. Under ideal circumstances, a single trial per condition is sufficient to evaluate cortical activation. However, because infant data present a low signal-to-noise ratio which is due to a number of reasons (e.g., physiological oscillations, instrument noise) and particularly to the presence of motion artifacts, a higher number of trials is required to compute statistical analyses (Wilcox \& Biondi, 2015). A minimum of three valid trials per experimental condition is often selected for statistical analyses (e.g. Lloyd-Fox et al., 2018). The activity evoked in single experimental trials is averaged to compute haemodynamic means for each condition and channel. Statistical analyses usually test activation elicited in one experimental condition relative to baseline (basic t-test), or difference in activation between two experimental conditions (paired t-test and ANOVA; Lloyd-Fox et al., 2010; Tak \& Ye, 2014).

One key advantage of fNIRS compared to other neuroimaging techniques, e.g. functional magnetic resonance imaging (fMRI), is its relatively high tolerance to movements. As long as fNIRS optodes (i.e. light sources and detectors) remain tightly fit to the head, fNIRS consents a quite ample range of movements to the child, which allows to test awake infants. It is noteworthy that, despite this quality, fNIRS infant 
recordings are often substantially corrupted by artifacts induced by movements. The field does not provide clear guidelines on how to correct for motion artifact (but see Chapter 2). Even so, fNIRS has been successfully used to investigate functional activation of the infant brain during a variety of cognitive and perceptual tasks. For instance, fNIRS has been applied to test infant face and facial expression processing, motor skills, language, numerosity abilities, and social touch (for a review on infant fNIRS applications see Wilcox \& Biondi, 2015). fNIRS also offers other advantages, e.g. it is not invasive, it can be portable, and it is relatively inexpensive compared to other neuroimaging techniques.

Nevertheless, fNIRS has also some limitations. As already mentioned, it can only give information about haemodynamic activity of cortical but not subcortical areas. Another critical point lies in the shape of the haemodynamic response observed in infants. Specifically, while in adults a stimulation is typically followed by an HRF with a canonical shape in activated brain regions (i.e., increase in $\mathrm{HbO}$ coupled with a decrease in $\mathrm{HbR}$ ), in infants also other patterns have been described, such as inverted responses (i.e., decrease in $\mathrm{HbO}$ and increase in $\mathrm{HbR}$ ) or simultaneous increase/decrease of $\mathrm{HbO}$ and $\mathrm{HbR}$ measures. Whereas the canonical HRF is interpreted as clear activation of the corresponding area, the non-canonical shapes are more difficult to explain. Some suggest that infant haemodynamic responses are critically influenced by several factors, e.g. the task design employed, the choice of baseline stimuli, the infants' age and the cortical regions involved (for more in depth discussion on infant HRF variability see Issard \& Gervain, 2018). Perhaps the major challenge in infant fNIRS studies is linked to its major advantage: how to deal with motion artifacts corrupting the recordings. As mentioned in the previous paragraph, fNIRS is less susceptible to movements compared to other techniques (e.g. ERPs and fMRI), but it is not invulnerable to motion artifacts. On the contrary, because infants move much more often than adults, infant fNIRS recordings are often substantially contaminated by artifacts. Motion artifacts are abrupt signal changes generated by the relative movement of the NIRS optodes with respect of the scalp and underling structures. If not properly corrected, these can affect the 
averaged HRF increasing the risk of false positives (i.e. mimic cortical activity) and false negatives (i.e. masking cortical activity), or simply affecting the response strength.

Currently, the main strategies to address this issue consist of (i) removing the trials affected by motion artifacts from the averaged HRF or (ii) use correction algorithms to recover the corrupted trials. In infant research, trial rejection (option ii) represents the most common strategy. Yet, this is not optimal since the low tolerance and compliance of the child in experimental settings allow the acquisition of only a limited number of trials per testing session. Thus, excluding trials could undermine the estimate of a reliable haemodynamic response and reduce the statistical power. On the other hand, artifact correction is currently based on guidelines derived from adult and simulateddata (Brigadoi et al., 2014; Cooper et al., 2012). Yet, motion artifacts in infant data are more diverse compared to those in adult data. More precisely, motion artifacts in infant recordings typically encompass a wider range of amplitude and frequency, and affect a larger portion of the recordings (high occurrence rate). All these characteristics make artifact correction more challenging in infant than in adult data, where artifacts can be more easily isolated. Thus, motion correction guidelines based on adult data might not be optimal for infant data. To date, only one study tested the efficacy of few correction methods in artifact correction of fNIRS infant data (Behrendt, Firk, Nelson, \& Perdue, 2018; i.e. Wavelet filtering, targeted Principal Component Analysis, and their combination). However, there are several other methods available for artifact correction (e.g. Spline interpolation, Wavelet Kurtosis, Spline Savitzky-Golay). The field requires a more comprehensive comparison to define which method best suits infant data. It is crucial to develop an adequate preprocessing stream that removes artifacts while preserving the true haemodynamic response before we can make accurate inferences about infant cortical activation.

The current thesis, specifically Chapter 2, aims to contribute to the field of fNIRS infant research by comparing the effects of most of the currently available correction methods, and of a novel approach (which combines two different correction techniques), on multiple infant datasets collected in different labs, using different task 
designs and sensors arrays. The aim of this chapter is to provide guidelines for infant data artifact correction, which in turn will also contribute to the standardization of the preprocessing pipelines across fNIRS infant studies.

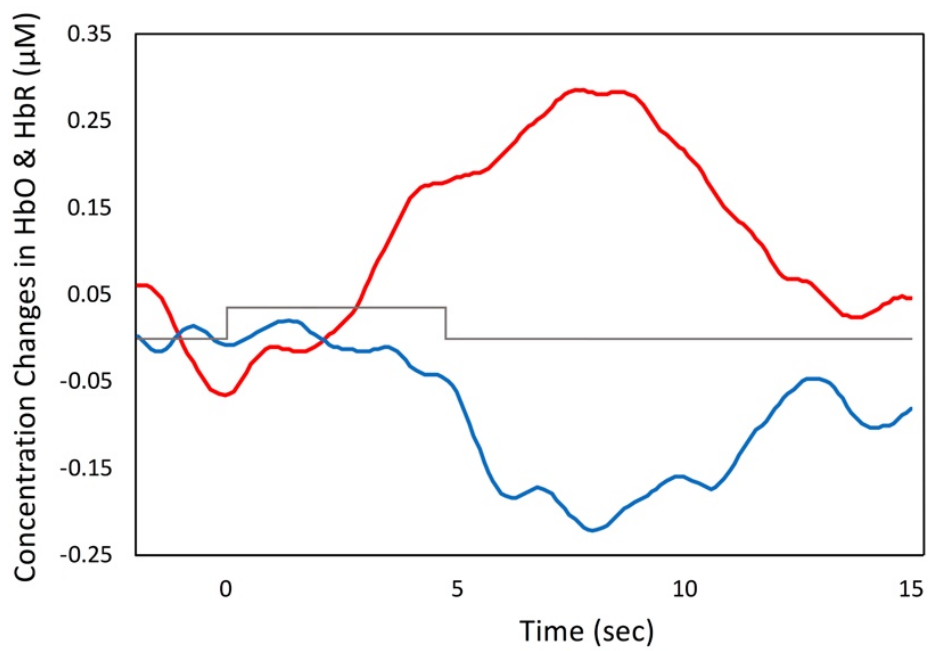

Figure 1.1 Example of typical haemodynamic response function (HRF) as recorded from a group of infants in our laboratory. The red line represents the change in $\mathrm{HbO}$, while the blue shows change in $\mathrm{HbR}$. The rectangle signals the experimental stimulation.

\section{Event-related potentials - ERPs}

While fNIRS measures a delayed consequence of neural activation (i.e. localized change in oxygen concentration), ERPs provide an instant estimate of neuronal activity (temporal resolution on the order of milliseconds) associated with specific brain mechanisms, such as those of face processing. To comprehend how the ERP method works, it is important to clarify how the brain operates. Neurons are electrically active brain cells that communicate with each other by means of action potentials. Action potentials are discrete electrical signals that trigger the release of neurotransmitters at the synapse, which is a near-contact area between neurons. When neurotransmitters bind to receptors of neurons that are on the other side of a synapse, electric current flows in those post-synaptic neurons (for a more in depth description of neuronal electrical activity see Luck, 2014). Under certain circumstances, the post-synaptic potentials of multiple neurons firing synchronously summate, making it possible to record them by electrodes placed on the scalp. Specifically, neural electrical activity is recorded between 
an active and a reference electrode (or site), the latter recording very low neuronal activity (e.g. ear lobes, or average voltage of all the scalp electrodes approximating $0 \mathrm{mV}$ ). Scalp recorded brain activity, also called electroencephalogram (EEG), mainly originates from cortical pyramidal neurons as they are aligned perpendicularly to the cortical surface and parallelly with each other (Luck, 2014). While EEG reflects brain's ongoing electrical activity, ERPs reflect transient changes in electrical activity time-locked to the onset of a stimulus or event (e.g. the view of a face). ERPs are collected from a number of trials and then averaged together to exclude signal unrelated to the experimental stimulation (Luck, 2014). In most ERP studies, an averaged ERP waveform is computed separately for each electrode (or set of electrodes) and for each experimental condition, first within each subject and subsequently across subjects. The waveform contains positive and negative deflections called ERP components (or peaks). For each component of interest, amplitudes or latencies elicited by different stimuli are typically compared. Amplitude or latency differences are commonly interpreted as differences in the number of active neurons or timing of the neuronal activation, respectively. It is worth noting that also other factors might influence these measures, such as the thickness of the skull, the reference electrode chosen, the variability of the latency of the signal across trials (for more in depth discussion see de Haan et al., 2007; Luck, 2014). Adult literature has clearly indicated that different ERP components reflect specific stages of perceptual processing or cognitive mechanisms (for reviews on components in adults see Luck, 2014). Yet, in infant research the link between ERP components and specific processes is less clear. For the aim of this thesis, I will review evidence of the components that have been related to the visual processing of faces in infancy.

In adults, one specific ERP component has been consistently found to discriminate faces vs. other stimuli, i.e. the occipito-temporal N170 (negative component occurring around $200 \mathrm{~ms}$ post-stimulus onset). Specifically, this component is thought to reflect early stages of face processing as its latency has been found shorter and its amplitude larger for faces compared to non-face stimuli (e.g., Rossion et al., 2000). Conversely, in infants three components displayed some sensitivity to faces (for a review see de Haan, 
Johnson, \& Halit, 2003): the occipito-temporal N290 and P400, and the fronto-central Negative central $(\mathrm{Nc})$. These components are thought to reflect different stages of face processing. The N290 (negative peak around $300 \mathrm{~ms}$ post-stimulus onset) and the P400 (positive peak around $400 \mathrm{~ms}$ post-stimulus onset) have been suggested to index early stages of visual processing of faces and possibly be the precursors of the adult facesensitive N170 (de Haan et al., 2003). The Nc (negative peak between 400-800ms poststimulus onset) is not face-specific but is relevant for face processing as it reflects a child's attention allocation and possibly looking preference (Courchesne, Ganz, \& Norcia, 1981; de Haan \& Nelson, 1997; de Haan et al., 2003; Reynolds, Courage, \& Richards, 2010). Although there are many studies that investigated infant ERPs to face processing, the field is undecided about which component consistently measures face processing across infancy. Notably, infant research reports conflicting results regarding the sensitivity of N290, P400 and Nc in measuring differences in the processing of human faces compared to other stimuli. For instance, while some studies show amplitude differences between face and other stimuli for all three components in infants between three and 12 months of age (e.g., see for the N290: de Haan, Pascalis, \& Johnson, 2002; Halit, de Haan, \& Johnson, 2003; McCleery, Akshoomoff, Dobkins, \& Carver, 2009; for the P400: Guy, Zieber, \& Richards, 2016; Halit et al., 2003; McCleery et al., 2009; for the Nc: Jones et al., 2016; McCleery et al., 2009; Webb, Long \& Nelson, 2005), other do not show significant differences (e.g., see for the N290: Cassia, Kuefner, Westerlund, \& Nelson, 2006; de Haan \& Nelson, 1999; Guy et al., 2016; for the P400: Cassia et al., 2006; de Haan \& Nelson, 1999; Halit, Csibra, Volein, \& Johnson, 2004; Halit et al., 2003; for the Nc: Cassia et al., 2006; de Haan \& Nelson, 1999; Guy et al., 2016). Hence, it is currently unclear which component is a robust measure of face processing across infancy.

The inconsistencies emerged from the literature might be linked to several aspects of the research designs adopted in the past. First, most of the ERP infant research reports statistics on small sample sizes $(\mathrm{N}<40)$, which might affect real effect sizes and hamper the replicability of findings. Next, infant research reports results based on one single 
narrow age-group or cross-sectional designs, which obscure the effect of development as well as the consistency in measuring face processing over time within the same infant group (but see Webb et al., 2005). Furthermore, most studies typically focus on a selection of the face-related components, making it difficult to compare the results relative to all three components across studies. Thus, the field calls for large longitudinal studies that clarify which infant component is a robust marker of face processing across infancy.

It is crucial to identify robust neural measures if we want to make meaningful interpretation of ERP effects of infant face processing. In Chapter 3, I make a step in this direction and evaluate to what extent each of the three infant face-related components (i.e. N290, P400, Nc) is a robust marker of face processing across infancy using longitudinal data from 80 infants tested at five and at 10 months of age with the same passive viewing task (i.e. visual presentation of neutral faces and houses).

Having described above which key methods are used to obtain infant brain correlates of face and facial expression processing, I will now outline the neural networks underlying such processing in the adult brain. The adult neural networks specific to face and facial expression processing are likely to emerge gradually over development (for a general theory on brain development see Karmiloff-Smith, 2015). The focus of my thesis is to advance our understanding of when and how these neural networks start to become functional during infancy (Chapter 3 and 4) and which factors might contribute to individual variation in their development (Chapter 5). 


\section{Face and facial expression processing in the adult brain}

Over the past decades, extensive research with adults identified the brain networks underlying face and facial expression processing (for reviews see de Haan \& Carver, 2013; Palermo \& Rhodes, 2007). These networks involve different neural pathways that interact with each other. Specifically, visual information about the face is initially transmitted via a subcortical and a cortical pathway. In the subcortical pathway, retinal visual inputs travel via fast-conducting magnocellular cells to the superior colliculus, the pulvinar, and the amygdala (Johnson, 2005; Johnson, Senju, \& Tomalski, 2015). This route largely relies on lower-spatial frequency information ${ }^{3}$ (LSF), i.e. visual information associated with the general configuration of elements within the face (Johnson, 2005). The subcortical pathway is thought to mediate orientation to faces, as well as rapid detection of faces and fear within faces (Johnson, 2005; 2015). It is hypothesized that this pathway allows a rapid but rough processing of the face before a slower yet detailed processing is carried out via the cortical pathway; moreover, the subcortical pathway might modulate the activity of the cortical pathway via bi-lateral projections (Johnson, 2005). In the cortical pathway, retinal visual information is transmitted via the striate cortex to the occipital face area (OFA; region part of the inferior occipital gyrus). The OFA is thought to be responsible for the early stages of visual face processing and to send inputs to the fusiform gyrus (FG, brain region that includes the fusiform face area) as well as to the superior temporal sulcus (STS) (for a review see de Haan \& Carver, 2013). The FG is mainly sensitive to the invariant aspects of faces and only to a lesser extent to emotional facial expressions (for more discussion see Said, Haxby, \& Todorov, 2011). Whereas the STS, which is also face-sensitive, is hypothesized to have a more prominent role in the processing of emotional facial expressions (Haxby, Hoffman, \& Gobbini, 2002; Said et al., 2011). Regions of the cortical pathway mainly receive higherspatial frequency information (HSF) that are carried by a slower parvocellular route (Johnson, 2005; Leppänen \& Nelson, 2009). The processing of HSF is thought to support

\footnotetext{
${ }^{3}$ Spatial frequencies are usually defined as the number of cycles per degree of visual angle (cpd) within an image (Morrison \& Schyns, 2001).
} 
the encoding of facial details, such as expression-related wrinkles (Johnson, 2005). Note that parts of the cortical pathway are likely to receive also some LSF information via the visual cortex (i.e. through a cortical magnocellular route; Nassi \& Callaway, 2009; Ungerleider \& Mishkin, 1982) as well as via projections from regions of the subcortical route (Vuilleumier \& Pourtois, 2007). For instance, previous research revealed that the face-sensitive FG shows an enhanced response to fearful expressions driven by LSF (e.g., Vuilleumier, Armony, Driver, \& Dolan, 2003); this effect is proposed to be mainly due to the projections from the amygdala, a subcortical area that is most sensitive to LSF within fearful faces as well (for more in depth discussion see Vuilleumier \& Pourtois, 2007).

The subcortical and the cortical pathways are also interconnected with other areas involved in other aspects of social processing. In particular, they display mutual connections with the orbito-frontal cortex (OFC), which is an area implicated in the recognition of emotions within faces and top-down modulation of perceptual processing (Leppänen \& Nelson, 2009).

To summarise, in the adult brain there are some regions that are simply activated by faces, mainly the OFA, the FG, and the STS (Haxby et al., 2002); and regions that are most sensitive to the emotional content of the face, that is the STS, the OFC, and the amygdala (Leppänen \& Nelson, 2009; Said et al., 2011). For convenience, I will refer to the latter set of areas as emotion processing network. How these neural networks develop and become adult-like is not fully understood. 


\section{Face processing in the infant brain}

Behavioural research with infants indicates that face processing abilities are present, to some extent, soon after birth and that critical changes occur within the first year of life. Indeed, already newborns show visual preference for faces and face-like patterns (Farroni et al., 2005; Johnson, Dziurawiec, Ellis, \& Morton, 1991). The nature of this 'face-bias' is still a matter of debate among developmental scientists. For instance, some interpret this evidence as proof that the brain is equipped with innate representations of faces, while others hypothesized that the face-bias is the result of a more general perceptual bias i.e. face configurations better suit the infant visual system (for a review see Johnson et al., 2015). Yet, a third influential theoretical account posits that the early orientation to faces is supported by the subcortical pathway, which ensures extensive exposure to faces and thereby might influence later cortical specialization to faces (Johnson, 2005; 2015). Despite these debates, it is currently accepted that the face-bias is present from early on. Furthermore, newborns' sensitivity to both pictures of faces and face-like patterns indicates that face processing is initially broadly tuned to face configurations in general. This idea is corroborated by evidence showing that newborns do not exhibit a spontaneous visual preference for human vs. monkey faces (Di Giorgio, Leo, Pascalis, \& Simion, 2012). Increased tuning to human faces has been reported during the second semester of life. For instance, between six and nine months of age infants can categorize faces based on ethnicity and can discriminate individual faces only within their own species (e.g., Anzures et al., 2010; Pascalis, de Haan, \& Nelson, 2002). Possibly, these changes in behaviour mirror an increase in cortical specialization to human faces in the first year of life. On this point, previous research used fNIRS and ERPs to uncover the neural underpinnings of face processing in infancy. While there are many ways to assess face processing skills, such as testing how infants process upright vs. inverted faces or familiar vs. unfamiliar faces, the focus of this thesis is on how infants process differences between human faces and other visual stimuli. Hence, the following paragraphs report fNIRS and ERPs findings only on this aspect of face processing. 
Some ERP studies suggest that infant cortical activity discriminates faces from other stimuli from around three months after birth onward (yet see a positron emission tomography study with 2-month-olds; Tzourio-Mazoyer et al., 2002). Recall ERPs mainly reflect cortical activity. Indeed, differences have been noted for components associated with early stages of visual processing of faces, the N290 and the P400, as well as for a component linked to attentional mechanisms, that is, the Nc. Specifically, the N290 amplitude has been found larger for faces vs. visual noise in 3-month-olds (Halit et al., 2004), vs. toys in 10-month-olds (infants at low-likelihood of developing ASD; McCleery et al., 2009), and vs. monkey faces in 3-, 6- and 12-month-olds (de Haan, Pascalis, \& Johnson, 2002; Halit et al., 2003). For the P400, studies report shorter latency for human faces compared to other stimuli in 6-, 10-, and 12-month-olds (de Haan \& Nelson, 1999; Halit et al., 2003; McCleery et al., 2009), as well as amplitude differences between human faces vs. monkey faces in 3-month-olds (Halit et al., 2003) and vs. toys in 4.5- to 12-month-olds (Guy et al., 2016; Jones et al., 2016; McCleery et al., 2009). With regard to the Nc, amplitude differences have been shown in 4- to 12-month-olds between faces and toys (Jones et al., 2016; McCleery et al., 2009; Webb et al., 2005). Although ERP patterns evoked by faces in infancy are still far from adult-like (for comparison between adult and infant ERP responses to faces see de Haan et al., 2003), infant ERPs do show to discriminate faces from other stimuli across the first year of life. However, since most of the studies reviewed above tested one age group or adopted a cross sectional design (but see one longitudinal study on the Nc by Webb et al., 2005), currently it is difficult to understand whether the processing of faces (vs. other stimuli) changes across infancy and at which level of visual processing (perceptual or attentional) this might appear. Hence, in Chapter 3, aside to the goal of testing which components are robust measures of face processing across infancy, I use the same large longitudinal ERP dataset to evaluate whether face processing changes between five and 10 months of age, and at which stage of visual processing such change occurs (i.e., perceptual: N290, P400; attentional: Nc). 
Further evidence of the involvement of cortical regions in infant face processing comes from fNIRS studies. In particular, face-specific activity has been recorded from occipital regions in 4-month-olds, and from temporal areas in 5- as well as in 7-montholds (for a review see Otsuka, 2014). While these findings might signal that parts of the adult cortical network of face processing are already functional in infants as young as five months, this inference is based on few studies selectively testing temporal or occipital areas in 4- to 5-month-olds (see research on occipital areas: Blasi et al., 2007; and temporal areas: Nakato et al., 2009; see also Kobayashi, Otsuka, Kanazawa, Yamaguchi, \& Kakigi, 2014 for non-significant face effects). Note that based on the position of the optodes on the infant scalp, the activity recorded by these studies might have partially originated from overlapping areas. Indeed, activity from occipital areas was recorded from channels placed over $\mathrm{O} 1-\mathrm{Oz}-\mathrm{O} 2$ sites relative to the 10-20 system (Blasi et al., 2007), while temporal activity was sampled from channels centred to T5/T6 (area close to STS; for research linking infant cortical areas with 10-20 system locations see Kabdebon et al., 2014) but reaching O1/O2 (close to OFA, Kabdebon et al., 2014). Thus, before we can conclude that occipital and temporal areas are both recruited in face processing already in 5-month-olds, it is necessary to replicate such effects by recording activity from both areas of the same infant sample. In addition, most fNIRS studies investigated face processing in older infants (for an overview see Otsuka, 2014). Yet, studying earlier developmental stages is relevant to explain the development of face processing. Therefore, Chapter 4 of the current thesis aims to contribute to the current literature of infant face processing by testing the status of cortical face-specific activation at five months of age. This study will help disentangling which areas, occipital and/or temporal, are involved in infant face processing at five months of age. The results of this chapter will also aid the selection of the cortical areas (or optode locations) for future longitudinal studies aiming to track individual differences in the development of face processing. 


\section{Facial expression processing in the infant brain}

Behavioural studies using preferential looking or habituation paradigms suggest that infants can discriminate between some emotional facial expressions soon after birth. For instance, newborns only few days old preferentially look at happy over fearful faces (Farroni, Menon, Rigato, \& Johnson, 2007), 3-month-olds discriminate smiling from frowning and surprised faces (Barrera \& Maurer, 1981; Young-Browne, Rosenfeld, \& Horowitz, 1977), 4- and 7-month-olds look longer at happy over angry and neutral faces (Grossmann, Striano, \& Federici, 2007; LaBarbera, Izard, Vietze, \& Parisi, 1976; Wilcox \& Clayton, 1968). While this early preference for happy faces over other facial expressions could be seen as an indication of innateness, or at least early onset, of facial expression discrimination (Ekman, Friesen, \& Ellsworth, 2013), other researchers suggest that it rather results from the predominant experience infants have with adults' happy facial expressions from early on (Farroni et al., 2007; Vaish, Grossman, \& Woodward, 2003). According to the latter hypothesis, infants of few months of age could discriminate the emotional expressions that they more often encounter (i.e. happy), but might not be yet able to discriminate among those that they do not often see (e.g. fear). In line with such perspective, infants show to discriminate basic emotional facial expressions and have a bias towards fearful faces between five-seven months of age (for a review see Leppänen \& Nelson, 2009; Peltola, Hietanen, Forssman, \& Leppänen, 2013). This period coincides to when they start to crawl and encounter threats in the environment (e.g. electricity sockets), which in turn prompts more frequent negative facial expressions in their caregivers. Within this period, infants show to recognize and categorize emotional facial expressions also across variations in intensity. For instance, studies using faces that are morphed in a continuous way between two emotions report that at seven months of age infants could categorize happy vs. sad and fearful vs. happy faces (Kotsoni, de Haan, \& Johnson, 2001; Leppänen, Richmond, Vogel-Farley, Moulson, \& Nelson, 2009). Yet other studies suggest that perceptual categorization is already possible at five months after birth for certain contrasts. For example, 5-montholds show categorial perception of (levels of) happy faces when tested against fearful 
faces (Bornstein \& Arterberry, 2003), and demonstrate sensitivity to the categorical boundary between facial expressions within the same emotional valence, i.e. positive (happiness and surprise) or negative (sadness and disgust, sadness and anger; White et al., 2019). In sum, these results suggest that starting from five months of age infants might be able to discriminate between many different emotional facial expressions. In the current thesis, I will focus on the neural network underlying the discrimination between facial expressions of different valence (e.g. happy vs. fearful).

According to a well-known developmental model of facial expression processing (Leppänen \& Nelson, 2009), the onset of these emotion-related abilities reflects the functional activation of the emotion processing network. This model posits that the network becomes functional between five and seven months of age and is biased for the processing of salient signals within faces (e.g. fear); the network might be further shaped by individual experiences with emotional facial expressions. Currently, there is limited direct knowledge about which areas of the emotion processing network are involved in facial expression processing in infancy. Most research on infant neural processing of facial expressions has been conducted with 7- to 10-month-olds using ERPs. Within this period, infants show to discriminate between fearful and neutral or happy facial expressions (for an overview see van den Boomen, Munsters, \& Kemner, 2019). In particular, amplitude differences between these facial expressions have been found for the face-sensitive perceptual components N290/P400 as well as for the attentional component Nc. Hence, ERP results suggest that the emotion processing network is active at this age. However, some ERP studies with younger infants suggest an earlier activation of the network. Specifically, three out of five ERP studies conducted on 4- to 5-month-olds report differential activity between happy and fearful faces at both perceptual and attentional components (Rigato, Farroni, \& Johnson, 2009; Xie, McCormick, Westerlund, Bowman, \& Nelson, 2019; Yrttiaho, Forssman, Kaatiala, \& Leppänen, 2014), while the other two report no significant differences (Hoehl \& Striano, 2010; Peltola, Leppänen, Mäki, \& Hietanen, 2009). 
Only very recently has fNIRS been used to investigate differential cortical activity evoked by facial expressions. In particular, one study suggested that at seven months, the STS is involved in the processing of angry (right STS) and happy faces (left STS; Nakato, Otsuka, Kanazawa, Yamaguchi, \& Kakigi, 2011). Furthermore, pictures or videos of happy faces activate the OFC in 7- to 13-month-olds (Fox, Wagner, Shrock, Tager-Flusberg, \& Nelson, 2013; Minagawa-Kawai et al., 2009; Ravicz, Perdue, Westerlund, Vanderwert, \& Nelson, 2015). Yet, it is unclear whether the emotion processing network is active already at five months, an age at which some behavioural and ERP studies suggest that facial expression discrimination might be already present.

In Chapter 4, I aim to contribute to the current understanding of when the emotion processing network becomes active in infancy by testing whether and which cortical areas of this network is differentially activated by fearful and happy faces at five months of age.

\section{Factors influencing face and facial expression processing early in life}

Although common brain networks are involved in face and facial expression processing in almost all infants, the sensitivity of these systems can vary substantially across individuals. Defining the factors that affect such processing early in life would contribute to our understanding of the typical development of face and facial expression processing, and most importantly would aid the early identification of the individuals that deviate from the typical patterns. In this context, a crucial factor is the genetic likelihood for ASD (for a definition of genetic likelihood of ASD see Box1). ASD is a neurodevelopmental disorder characterized by impairments in social communication and the presence of repetitive behaviours and restricted interests (American Psychiatric Association, 2013). Possibly, the difficulties in social communication displayed by individuals with ASD are linked to difficulties in the processing of social stimuli, such as faces and facial expressions. Indeed, previous ERP studies showed that adults with ASD as well as infants at a higher genetic likelihood for ASD, hereinafter referred to as 
Higher-Likelihood (HL) infants ${ }^{4}$, differ in the neural responses to faces and facial expressions when compared to neurotypical controls (for studies on face processing in HL infants see McCleery at al., 2009; in adults with ASD see McPartland, Dawson, Webb, Panagiotides, \& Carver, 2004; for a review on facial expression processing in individuals of all ages with ASD see Black et al., 2017). Hence, this genetic factor influences both face and facial expression processing at different stages of development. According to a widely accepted account of neurocognitive development (the so-called neuroconstructivism; D'Souza \& Karmiloff-Smith, 2016), even small differences early in life may lead to cascading effects in several domains over development. Therefore, differences in the processing of faces or facial expressions in infancy might contribute to difficulties in social communication as well as affect learning opportunities later in life. Consequently, it is crucial to accurately define the nature of such processing differences during infancy so that future research can build new interventions tailored to support the efficient development of face and facial expression processing in individuals with a higher likelihood for ASD. In the current thesis I will focus on one aspect that was found to influence facial expression processing in children with ASD compared to controls: i.e. the visual processing of facial details and global information (e.g., Vlamings, Jonkman, van Daalen, van der Gaag, \& Kemner, 2010). Recall the processing of facial details is mainly supported by the processing of HSF, while global information processing by LSF encoding (see previous paragraph on face and facial expression processing in the adult brain). A previous ERP study showed that facial expression processing was driven by facial details (or HSF) in toddlers with ASD and by global facial information (or LSF) in controls (Vlamings et al., 2010). Group differences in the processing of facial expressions were reported for the amplitude of the P100, a component that indexes an earlier stage of visual processing compared to the facespecific N170, which suggests very early perceptual differences. These results indicate that the processing of specific visual facial information and the genetic predispositions

\footnotetext{
${ }^{4}$ The literature typically uses the term 'risk' for ASD, instead I chose to use the term 'likelihood' in line with the preferences of the autistic community (Fletcher-Watson et al., 2017).
} 
(ASD) interact and contribute to individual differences in the processing of facial expressions during childhood. Yet, it is unclear if these factors interact also at an earlier developmental stage, e.g. in infancy.

In Chapter 5, I test whether HL 10-month-old infants differ from controls in the processing of global/detailed facial information (i.e. LSF/HSF) when discriminating between emotional facial expressions.

\section{Box1 Reason for studying High-Likelihood siblings}

Because ASD is a complex disorder with a heterogenous phenotype and genotype it is difficult to define the aetiology or developmental pathway leading to the condition (Jones, Gliga, Bedford, Charman, \& Johnson, 2014). Hence, recently developmental researchers started investigating more simplified characteristics of the disorder, i.e. its endophenotypes. Endophenotypes are biological markers that may exist at any phenotypic level between gene and behaviour (Gottesman \& Gould, 2003). These markers should appear in the first years of life prior to the clinical symptoms and may be present in individuals with ASD as well as in their unaffected siblings. Given these characteristics, a growing number of studies is investigating endophenotypes in infants siblings of children with ASD. These infants have approximately $20 \%$ chance of receiving an ASD diagnosis (Ozonoff et al., 2011), and for this reason are called high-likelihood. Notably, despite the majority of HL infants will not develop ASD, around 10-20\% might still display subclinical traits of ASD (Messinger et al., 2013). The design of studies targeting HL infants typically includes also infants who do not have a sibling with ASD (called low-likelihood infants; LL) as control group; note that population prevalence is around 1\% (Baird et al., 2006). Comparisons between HL and LL infants provide crucial information about ASD traits, or endophenotypes. 


\section{Goals and Outline of the thesis}

The ultimate aim of this thesis is to contribute to the current understanding of how infant brains process faces and facial expressions. To achieve this general aim, my research focuses on two goals: (1) contribute to the definition of robust neural measures of infant face processing; (2) extend current knowledge about how face and facial expression processing typically develop in infancy and which factors might contribute to individual differences in facial expression processing. The target age of my research is between five and 10 months of age as this period has been found pivotal for the development of face and facial expression processing. As regard to the first goal, in Chapter 2 and 3 I will address two pending issues in infant fNIRS and ERP research. Specifically, Chapter 2 aims to provide general guidelines for the preprocessing of infant fNIRS data, with a particular focus on how to correct motion artifacts. To this end, I will compare the efficacy of most of the motion correction techniques available, plus a novel approach, on multiple infant fNIRS datasets. Next, in Chapter 3, I turn to ERPs and test to what extent each component previously defined as face-related (i.e., N290, P400, Nc) is a robust marker of face processing across infancy, specifically between five and 10 months of age. Within Chapter 3, I will also tap into the second goal of this thesis. In particular, in this chapter I will evaluate whether a basic aspect of face processing, i.e. differential processing of faces and non-face stimuli, undergoes developmental changes between five and 10 months after birth and at which stage of visual processing such changes might occur (i.e., early perceptual: N290, P400; attentional: Nc). Next, in Chapter 4 I will test which cortical regions are recruited for face and facial expression processing at five months of age using fNIRS. At last, in Chapter 5 I will focus on two factors that might influence facial expression processing in infancy. Specifically, I will investigate whether and how the genetic likelihood of developing ASD and the visual processing of facial details and global information affect infants' ability to process emotions within faces at 10 months of age. 
Together these studies will contribute to our understanding of how the infant brain develops the ability to process faces and facial expressions over the first year of life. As faces convey crucial cues for successful social communication, knowledge on the typical development of these processes helps understanding why changes in social behaviour occur early in life in the typical range and why some individuals might deviate from the typical developmental trajectory (e.g. in ASD). Furthermore, results of this research might provide useful information for the tailoring of new early interventions aiming to improve facial expression processing, and consequently social communication, in children with ASD as well as typically-developing. An overview of the chapters and concepts discussed in this thesis is provided in Figure 1.2.

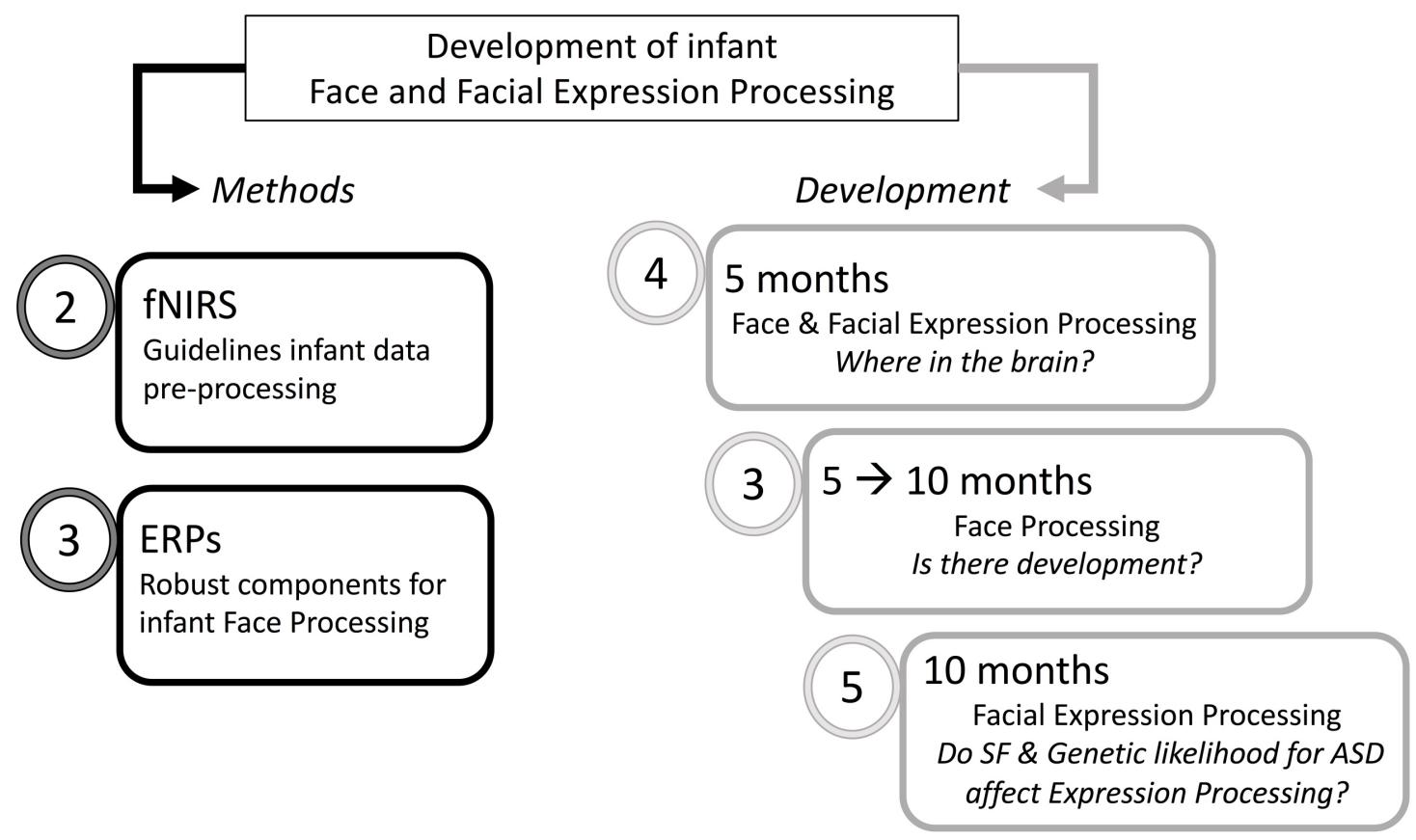

Figure 1.2 Overview of the chapters and concepts discussed in the present thesis. Numbers in the circles represent the chapters. 


\section{References}

Anzures, G., Quinn, P. C., Pascalis, O., Slater, A. M., \& Lee, K. (2010). Categorization, categorical perception, and asymmetry in infants' representation of face race. Developmental Science, 13(4), 553-564. https://doi.org/10.1111/j.14677687.2009.00900.x

Arichi, T., Fagiolo, G., Varela, M., Melendez-Calderon, A., Allievi, A., Merchant, N., ... \& Edwards, A. D. (2012). Development of BOLD signal hemodynamic responses in the human brain. NeuroImage, 63(2), 663-673. https://doi.org/10.1016/j.neuroimage.2012.06.054

Arridge, S. R., Cope, M., \& Delpy, D. T. (1992). The theoretical basis for the determination of optical pathlengths in tissue: Temporal and frequency analysis. Physics in Medicine and Biology, 37(7), 1531-1560. http://doi.org/10.1088/0031-9155/37/7/005

Baird, G., Simonoff, E., Pickles, A., Chandler, S., Loucas, T., Meldrum, D., \& Charman, T. (2006). Prevalence of disorders of the autism spectrum in a population cohort of children in South Thames: the Special Needs and Autism Project (SNAP). The lancet, 368(9531), 210-215. https://doi.org/10.1016/S0140-6736(06)69041-7

Barrera, M. E., \& Maurer, D. (1981). The perception of facial expressions by the three-monthold. Child development, 203-206. http://dx.doi.org/10.2307/1129231

Behrendt, H. F., Firk, C., Nelson, C. A., \& Perdue, K. L. (2018). Motion correction for infant functional near-infrared spectroscopy with an application to live interaction data. Neurophotonics, 5(01), 1. https://doi.org/10.1117/1.NPh.5.1.015004

Black, M. H., Chen, N. T. M., Iyer, K. K., Lipp, O. V, Bölte, S., Falkmer, M., ... \& Girdler, S. (2017). Neuroscience and Biobehavioral Reviews Mechanisms of facial emotion recognition in autism spectrum disorders: Insights from eye tracking and electroencephalography. Neuroscience and Biobehavioral Reviews, 80, 488-515. http://doi.org/10.1016/j.neubiorev.2017.06.016

Blasi, A., Fox, S., Everdell, N., Volein, A., Tucker, L., Csibra, G., ... Elwell, C. E. (2007). Investigation of depth dependent changes in cerebral haemodynamics during face perception in infants. Physics in Medicine \& Biology, 52(23), 6849-6864. https://doi.org/10.1088/0031-9155/52/23/005

Bornstein, M. H., \& Arterberry, M. E. (2003). Recognition, discrimination and categorization of smiling by 5-month-old infants. Developmental Science, 6(5), 585-599. https://doi.org/10.1111/1467-7687.00314

Brigadoi, S., Ceccherini, L., Cutini, S., Scarpa, F., Scatturin, P., Selb, J., ... Cooper, R. J. (2014). Motion artifacts in functional near-infrared spectroscopy: A comparison of motion correction techniques applied to real cognitive data. NeuroImage, 85, 181-191. https://doi.org/10.1016/j.neuroimage.2013.04.082 
Cassia, V. M., Kuefner, D., Westerlund, A., \& Nelson, C. A. (2006). A behavioural and ERP investigation of 3-month-olds' face preferences. Neuropsychologia, 44(11), 2113-2125. https://doi.org/10.1016/j.neuropsychologia.2005.11.014

Cooper, R., Selb, J., Gagnon, L., Phillip, D., Schytz, H. W., Iversen, H. K., ... \& Boas, D. A. (2012). A systematic comparison of motion artifact correction techniques for functional near-infrared spectroscopy. Frontiers in Neuroscience, 6, 147. https://doi.org/10.3389/fnins.2012.00147

Courchesne, E., Ganz, L., \& Norcia, A. M. (1981). Event-related brain potentials to human faces in infants. Child development, 52, 804-811. http://doi.org/10.2307/1129080

D'Souza, H., \& Karmiloff-Smith, A. (2016). Neurodevelopmental disorders. Wiley Interdisciplinary Reviews: Cognitive Science, 8(1-2), e1398. http://doi.org/10.1002/wcs.1398

de Haan, M, \& Nelson, C. A. (1999). Brain activity differentiates faces and object processing in 6-month-old infants. Developmental Psychology, 35(4), 1113-1121.

de Haan, M. (2007). Current and future directions in infant electrophysiology. In: de Haan M. (Ed.), Infant EEG and event-related potentials, (pp. 305-314). New York, NY, US: Psychology Press.

de Haan, M. D., Pascalis, O., \& Johnson, M. H. (2002). Specialization of neural mechanisms underlying face recognition in human infants. Journal of Cognitive Neuroscience, 14(2), 199-209. https://doi.org/10.1162/089892902317236849

de Haan, M., \& Carver, L. J. (2013). Development of brain networks for visual socialemotional information processing in infancy. In Legerstee M., Haley D.W., \& Bornstein, M. H. (Eds.), The infant mind: Origins of the social brain, (pp 123-145). New York, NY, US: Guilford Publications.

de Haan, M., \& Nelson, C. A. (1997). Recognition of the mother's face by six-month-old infants: A neurobehavioral study. Child development, 68(2), 187-210.

de Haan, M., Johnson, M. H., \& Halit, H. (2003). Development of face-sensitive event-related potentials during infancy: a review. International Journal of Psychophysiology, 51(1), 4558. https://doi.org/10.1016/S0167-8760(03)00152-1

de Haan, M., Pascalis, O., \& Johnson, M. H. (2002). Specialization of neural mechanisms underlying face recognition in human infants. Journal of cognitive neuroscience, 14(2), 199-209.

DeBoer, T., Scott, L. S., \& Nelson, C. A. (2007). Methods for acquiring and analyzing infant event-related potentials. In de Haan M. (Ed.), Infant EEG and event-related potentials, (pp. 5-37). New York, NY, US: Psychology Press. 
Delpy, D. T., Cope, M., van der Zee, P., Arridge, S. R., Wray, S., \& Wyatt, J. S. (1988).

Estimation of optical pathlength through tissue from direct time of flight measurement.

Physics in Medicine \& Biology, 33(12), 1433. https://doi.org/10.1088/0031-

$9155 / 33 / 12 / 008$

Di Giorgio, E., Leo, I., Pascalis, O., \& Simion, F. (2012). Is the face-perception system humanspecific at birth?. Developmental psychology, 48(4), 1083.

http://dx.doi.org/10.1037/a0026521

Ekman, P., Friesen, W. V., \& Ellsworth, P. (2013). Emotion in the human face: Guidelines for research and an integration of findings. New Your, NY, US: Pergamon Press Inc.

Elwell, C. E. (1995). A Practical Users Guide to Near Infrared Spectroscopy. (Hamamatsu Photonics KK, Ed.) (Hamamatsu). London (UK): Hamamatsu Photonics KK.

Farroni, T., Johnson, M. H., Menon, E., Zulian, L., Faraguna, D., \& Csibra, G. (2005). Newborns' preference for face-relevant stimuli: Effects of contrast polarity. Proceedings of the National Academy of Sciences, 102(47), 17245-17250.

https://doi.org/10.1073/pnas.0502205102

Farroni, T., Menon, E., Rigato, S., \& Johnson, M. H. (2007). The perception of facial expressions in newborns. European Journal of Developmental Psychology, 4(1), 2-13. http://dx.doi.org/10.1080/17405620601046832

Fletcher-Watson, S., A picella, F., Auyeung, B., Beranova, S., Bonnet-Brilhault, F., Canal-Bedia, R., ... \& Farroni, T. (2017). Attitudes of the autism community to early autism research. Autism, 21(1), 61-74. https://doi.org/10.1177/1362361315626577

Fox, S. E., Wagner, J., Shrock, C. L., Flusberg, H. T., \& Nelson, C. A. (2013). Neural processing of facial identity and emotion in infants at high-risk for autism spectrum disorders. Frontiers in Human Neuroscience, 7, 89. https://doi.org/10.3389/fnhum.2013.00089

Gottesman, I. I., \& Gould, T. D. (2003). The endophenotype concept in psychiatry: etymology and strategic intentions. American Journal of Psychiatry, 160(4), 636-645. https://doi.org/10.1176/appi.ajp.160.4.636

Grossmann, T., Striano, T., \& Friederici, A. D. (2007). Developmental changes in infants' processing of happy and angry facial expressions: A neurobehavioral study. Brain and cognition, 64(1), 30-41. http://dx.doi.org/10.1016/j.bandc.2006.10.002

Guy, M. W., Zieber, N., \& Richards, J. E. (2016). The Cortical Development of Specialized Face Processing in Infancy. Child Development, 87(5), 1581-1600. https://doi.org/10.1111/cdev.12543

Halit, H., Csibra, G., Volein, A., \& Johnson, M. H. (2004). Face-sensitive cortical processing in early infancy. Journal of Child Psychology and Psychiatry, 45(7), 1228-1234.

http://doi.org/ 10.1111/j.1469-7610.2004.00321.x 
Halit, H., de Haan, M., \& Johnson, M. H. (2003). Cortical specialisation for face processing: Face-sensitive event-related potential components in 3- and 12-month-old infants. NeuroImage, 19(3), 1180-1193. https://doi.org/10.1016/S1053-8119(03)00076-4

Haxby, J. V., Hoffman, E. A., \& Gobbini, M. I. (2002). Human neural systems for face recognition and social communication. Biological Psychiatry, 51(1), 59-67. https://doi.org/10.1016/S0006-3223(01)01330-0

Hoehl, S., Striano, T., 2010b. The development of emotional face and eye gaze processing. Developmental Science, 13(6), 813-825. https://doi.org/10.1111/j.14677687.2009.00944.x.

Issard, C., \& Gervain, J. (2018). Variability of the hemodynamic response in infants: Influence of experimental design and stimulus complexity. Developmental cognitive neuroscience, 33, 182-193. https://doi.org/10.1016/j.dcn.2018.01.009

Jacques, S. L. (2013). Optical properties of biological tissues: a review. Physics in Medicine \& Biology, 58(11), R37. https://doi.org/10.1088/0031-9155/58/11/R37

Johnson, M. H. (2005). Subcortical face processing. Nature Reviews Neuroscience, 6(10), 766774. https://doi.org/10.1038/nrn1766

Johnson, M. H., Dziurawiec, S., Ellis, H., \& Morton, J. (1991). Newborns' preferential tracking of face-like stimuli and its subsequent decline. Cognition, 40(1-2), 1-19.

Johnson, M. H., Senju, A., \& Tomalski, P. (2015). Neuroscience and Biobehavioral Reviews The two-process theory of face processing : Modifications based on two decades of data from infants and adults. Neuroscience and Biobehavioral Reviews, 50, 169-179. https://doi.org/10.1016/j.neubiorev.2014.10.009

Jones, E. J. H., Gliga, T., Bedford, R., Charman, T., \& Johnson, M. H. (2014). Neuroscience and Biobehavioral Reviews Developmental pathways to autism : A review of prospective studies of infants at risk. Neuroscience and Biobehavioral Reviews, 39, 1-33. https://doi.org/10.1016/j.neubiorev.2013.12.001

Jones, E. J., Venema, K., Earl, R., Lowy, R., Barnes, K., Estes, A., ... \& Webb, S. J. (2016). Reduced engagement with social stimuli in 6-month-old infants with later autism spectrum disorder: a longitudinal prospective study of infants at high familial risk. Journal of neurodevelopmental disorders, 8(1), 7. http://doi.org/10.1186/s11689016-9139-8

Kabdebon, C., Leroy, F., Simmonet, H., Perrot, M., Dubois, J., \& Dehaene-Lambertz, G. (2014). Anatomical correlations of the international 10-20 sensor placement system in infants. NeuroImage, 99, 342-356. https://doi.org/10.1016/j.neuroimage.2014.05.046

Kabdebon, C., Leroy, F., Simmonet, H., Perrot, M., Dubois, J., \& Dehaene-Lambertz, G. (2014). Anatomical correlations of the international 10-20 sensor placement system in infants. NeuroImage, 99, 342-356. http://dx.doi.org/10.1016/j.neuroimage.2014.05.046 
Karmiloff-Smith, A. (2015). An alternative to domain-general or domain-specific frameworks for theorizing about human evolution and ontogenesis. AIMS Neuroscience, 2(2), 91104. https://doi.org/10.3934/Neuroscience.2015.2.91

Kobayashi, M., Otsuka, Y., Kanazawa, S., Yamaguchi, M. K., \& Kakigi, R. (2014). The processing of faces across non-rigid facial transformation develops at 7 month of age: a fNIRS-adaptation study. BMC Neuroscience, 15(1), 81.

Kotsoni, E., de Haan, M., \& Johnson, M. H. (2001). Categorical perception of facial expressions by 7-month-old infants. Perception, 30(9), 1115-1125. https://doi.org/10.1068/p3155

LaBarbera, J. D., Izard, C. E., Vietze, P., \& Parisi, S. A. (1976). Four-and six-month-old infants' visual responses to joy, anger, and neutral expressions. Child development, 535538.

Leppänen, J. M., \& Nelson, C. A. (2009). Tuning the developing brain to social signals of emotions. Nature Reviews Neuroscience, 10(1), 37-47. https://doi.org/10.1038/nrn2554

Leppänen, J. M., Richmond, J., Vogel-Farley, V. K., Moulson, M. C., \& Nelson, C. A. (2009). Categorical representation of facial expressions in the infant brain. Infancy, 14(3), 346362. https://doi.org/10.1080/15250000902839393

Lloyd-Fox, S., Blasi, A., \& Elwell, C. E. (2010). Illuminating the developing brain: The past, present and future of functional near infrared spectroscopy. Neuroscience and Biobehavioral Reviews, 34(3), 269-284. https://doi.org/10.1016/j.neubiorev.2009.07.008

Lloyd-Fox, S., Blasi, A., Pasco, G., Gliga, T., Jones, E. J. H., Murphy, D. G. M., .. Yemane, F. (2018). Cortical responses before 6 months of life associate with later autism. European Journal of Neuroscience, 47(6), 736-749. http://doi.org/10.1111/ejn.13757

Lloyd-Fox, Sarah, Richards, J. E., Blasi, A., Murphy, D. G. M., Elwell, C. E., \& Johnson, M. H. (2014). Coregistering functional near-infrared spectroscopy with underlying cortical areas in infants. Neurophotonics, 1(2), 025006. https://doi.org/10.1117/1.NPh.1.2.025006

Luck, S. J. (2014). An introduction to the event-related potential technique. 2nd Edn. Cambridge, MA: MIT Press.

McCleery, J. P., Akshoomoff, N., Dobkins, K. R., \& Carver, L. J. (2009). Atypical Face Versus Object Processing and Hemispheric Asymmetries in 10-Month-Old Infants at Risk for Autism. Biological Psychiatry, 66(10), 950-957. https://doi.org/10.1016/j.biopsych.2009.07.031

McPartland, J., Dawson, G., Webb, S. J., Panagiotides, H., \& Carver, L. J. (2004). Event-related brain potentials reveal anomalies in temporal processing of faces in autism spectrum disorder. Journal of Child Psychology and Psychiatry, 45(7), 1235-1245. https://doi.org/10.1111/j.1469-7610.2004.00318.x 
Messinger, D., Young, G. S., Ozonoff, S., Dobkins, K., Carter, A., Zwaigenbaum, L., ... \& Hutman, T. (2013). Beyond autism: a baby siblings research consortium study of highrisk children at three years of age. Journal of the American Academy of Child \& Adolescent Psychiatry, 52(3), 300-308. https://doi.org/10.1016/j.jaac.2012.12.011

Minagawa-Kawai, Y., Matsuoka, S., Dan, I., Naoi, N., Nakamura, K., \& Kojima, S. (2009). Prefrontal activation associated with social attachment: Facial-emotion recognition in mothers and infants. Cerebral Cortex, 19(2), 284-292.

https://doi.org/10.1093/cercor/bhn081

Morrison, D. J., \& Schyns, P. G. (2001). Usage of spatial scales for the categorization of faces, objects, and scenes. Psychonomic Bulletin \& Review, 8(3), 454-469.

https://doi.org/10.3758/BF03196180

Nakato, E., Otsuka, Y., Kanazawa, S., Yamaguchi, M. K., Watanabe, S., \& Kakigi, R. (2009). When do infants differentiate profile face from frontal face? A near-infrared spectroscopic study. Human Brain Mapping, 30(2), 462-472.

https://doi.org/10.1002/hbm.20516

Nakato, E., Otsuka, Y., Kanazawa, S., Yamaguchi, M., \& Kakigi, R. (2011). Distinct differences in the pattern of hemodynamic response to happy and angry facial expressions in infants-A near-infrared spectroscopic study. NeuroImage, 54(2), 1600-1606. https://doi.org/10.1016/j.neuroimage.2010.09.021

Nassi, J. J., \& Callaway, E. M. (2009). Parallel processing strategies of the primate visual system. Nature reviews neuroscience, 10(5), 360-372. http://dx.doi.org/10.1038/nrn2619.

Otsuka, Y. (2014). Face recognition in infants: A review of behavioral and near-infrared spectroscopic studies. Japanese Psychological Research, 56(1), 76-90. https://doi.org/10.1111/jpr.12024

Ozonoff, S., Young, G. S., Carter, A., Messinger, D., Yirmiya, N., Zwaigenbaum, L., ... \& Hutman, T. (2011). Recurrence risk for autism spectrum disorders: a Baby Siblings Research Consortium study. Pediatrics, 128(3), e488-e495.

Palermo, R., \& Rhodes, G. (2007). Are you always on my mind? A review of how face perception and attention interact. Neuropsychologia, 45(1), 75-92. https://doi.org/10.1016/j.neuropsychologia.2006.04.025

Pascalis, O., de Haan, M., \& Nelson, C. A. (2002). Is face processing species-specific during the first year of life?. Science, 296(5571), 1321-1323. https://doi.org/10.1126/science.1070223

Peltola, M. J., Hietanen, J. K., Forssman, L., \& Leppänen, J. M. (2013). The emergence and stability of the attentional bias to fearful faces in infancy. Infancy, 18(6), 905-926. https://doi.org/10.1111/infa.12013 
Peltola, M.J., Leppänen, J.M., Mäki, S., Hietanen, J.K., 2009. Emergence of enhanced attention to fearful faces between 5 and 7 months of age. Social cognitive and affective neuroscience, 4(2), 134-142. http://dx.doi.org/10.1093/scan/nsn046.

Ravicz, M. M., Perdue, K. L., Westerlund, A., Vanderwert, R. E., \& Nelson, C. A. (2015). Infants' neural responses to facial emotion in the prefrontal cortex are correlated with temperament: a functional near-infrared spectroscopy study. Frontiers in Psychology, 6, 922. https://doi.org/10.3389/fpsyg.2015.00922

Reynolds, G. D., Courage, M. L., \& Richards, J. E. (2010). Infant attention and visual preferences: Converging evidence from behavior, event-related potentials, and cortical source localization. Developmental Psychology, 46(4), 88.

http://dx.doi.org/10.1037/a0019670

Rigato, S., Farroni, T., \& Johnson, M. (2009). The shared signal hypothesis and neural responses to expressions and gaze in infants and adults. Social Cognitive and Affective Neuroscience, 5(1), 88-97. https://doi.org/10.1093/scan/nsp037

Rossion, B., Gauthier, I., Tarr, M. J., Despland, P., Bruyer, R., Linotte, S., \& Crommelinck, M. (2000). The N170 occipito-temporal component is delayed and enhanced to inverted faces but not to inverted objects: an electrophysiological account of face-specific processes in the human brain. Neuroreport, 11(1), 69-74.

https://doi.org/10.1097/00001756-200001170-00014

Said, C. P., Haxby, J. V., \& Todorov, A. (2011). Brain systems for assessing the affective value of faces. Philosophical Transactions of the Royal Society B: Biological Sciences, 366(1571), 1660-1670. https://doi.org/10.1098/rstb.2010.0351

Tak, S., \& Ye, J. C. (2014). Statistical analysis of fNIRS data: a comprehensive review. NeuroImage, 85, 72-91. http://dx.doi.org/10.1016/j.neuroimage.2013.06.016

Tzourio-Mazoyer, N., De Schonen, S., Crivello, F., Reutter, B., Aujard, Y., \& Mazoyer, B. (2002). Neural correlates of woman face processing by 2-month-old infants. NeuroImage, 15, 454-461. http://dx.doi.org/ 10.1006/nimg.2001.0979

Ungerleider L, Mishkin M (1982): Two cortical visual systems. In: Ingle D, Goodale M, Mansfield R, (Eds.), Analysis of Visual Behavior (pp 549-586). Cambridge, MA: MIT Press

Vaish, A., Grossmann, T., \& Woodward, A. (2008). Not all emotions are created equal: the negativity bias in social-emotional development. Psychological bulletin, 134(3), 383. https://doi.org/10.1037/0033-2909.134.3.383

van den Boomen, C., Munsters, N., \& Kemner, C. (2019). Emotion processing in the infant brain: The importance of local information. Neuropsychologia, 126, 62-68. https://doi.org/10.1016/j.neuropsychologia.2017.09.006 
Villringer, A., \& Chance, B. (1997). Non-invasive optical spectroscopy and imaging of human brain function. Trends in Neurosciences, 20(10), 435-442. http://doi.org/10.1016/S01662236(97)01132-6

Vlamings, P. H. J. M., Jonkman, L. M., van Daalen, E., van der Gaag, R. J., \& Kemner, C. (2010). Basic abnormalities in visual processing affect face processing at an early age in autism spectrum disorder. Biological Psychiatry, 68(12), 1107-1113. http://doi.org/10.1016/j.biopsych.2010.06.024

Vuilleumier, P., \& Pourtois, G. (2007). Distributed and interactive brain mechanisms during emotion face perception: Evidence from functional neuroimaging. Neuropsychologia, 45(1), 174-194. https://doi.org/10.1016/j.neuropsychologia.2006.06.003

Vuilleumier, P., Armony, J. L., Driver, J., \& Dolan, R. J. (2003). Distinct spatial frequency sensitivities for processing faces and emotional expressions. Nature Neuroscience, 6(6), 624. https://doi.org/10.1038/nn1057

Webb, S. J., Long, J. D., \& Nelson, C. A. (2005). A longitudinal investigation of visual eventrelated potentials in the first year of life. Developmental Science, 8(6), 605-616. https://doi.org/10.1111/j.1467-7687.2005.00452.x

White, H., Chroust, A., Heck, A., Jubran, R., Galati, A., \& Bhatt, R. S. (2019). Categorical Perception of Facial Emotions in Infancy. Infancy, 24(2), 139-161. https://doi.org/10.1111/infa.12275

Wilcox, B. M., \& Clayton, F. L. (1968). Infant visual fixation on motion pictures of the human face. Journal of Experimental Child Psychology, 6(1), 22-32. https://doi.org/10.1016/0022-0965(68)90068-4

Wilcox, T., \& Biondi, M. (2015). fNIRS in the developmental sciences. Wiley Interdisciplinary Reviews: Cognitive Science, 6(3), 263-283. https://doi.org/10.1002/wcs.1343

Xie, W., McCormick, S. A., Westerlund, A., Bowman, L. C., \& Nelson, C. A. (2019). Neural correlates of facial emotion processing in infancy. Developmental science, 22(3), e12758. https://doi.org/10.1111/desc.12758

Young-Browne, G., Rosenfeld, H. M., \& Horowitz, F. D. (1977). Infant discrimination of facial expressions. Child Development, 48 (2) 555-562.

Yrttiaho, S., Forssman, L., Kaatiala, J., \& Leppänen, J. M. (2014). Developmental precursors of social brain networks: The emergence of attentional and cortical sensitivity to facial expressions in 5 to 7 months old infants. PLoS ONE, 9(6). https://doi.org/10.1371/journal.pone.0100811 



\title{
Recommendations for motion correction of infant fNIRS data applicable to multiple data sets and acquisition systems
}

\author{
Renata Di Lorenzo \\ Laura Pirazzoli \\ Anna Blasi \\ Chiara Bulgarelli \\ Yoko Hakuno \\ Yasuyo Minagawa \\ Sabrina Brigadoi
}

Published as:

Di Lorenzo, R., Pirazzoli, L., Blasi, A., Bulgarelli, C., Hakuno, Y., Minagawa, Y., \& Brigadoi, S. (2019). Recommendations for motion correction of infant fNIRS data applicable to multiple data sets and acquisition systems. NeuroImage, 200, 511-527. http://doi.org/10.1016/j.neuroimage.2019.06.056

Author contribution:

$\mathrm{RDL}, \mathrm{LP}, \mathrm{AB}$ and $\mathrm{SB}$ contributed to the conception of the study. RDL processed dataset 2 and 3, LP dataset 2 and $4, A B$ and SB dataset 1. RDL and LP wrote the first draft of the manuscript. $\mathrm{RDL}, \mathrm{LP}, \mathrm{AB}$ and $\mathrm{SB}$ substantially contributed in the revision of the manuscript. All authors read and approved the submitted version. RDL and LP equally contributed to this work. 


\begin{abstract}
Despite motion artifacts are a major source of noise in fNIRS infant data, how to approach motion correction in this population has only recently started to be investigated. Homer2 offers a wide range of motion correction methods and previous work on simulated and adult data suggested the use of Spline interpolation and Wavelet filtering as optimal methods for the recovery of trials affected by motion. However, motion artifacts in infant data differ from those in adults' both in amplitude and frequency of occurrence. Therefore, artifact correction recommendations derived from adult data might not be the optimal for infant data. We hypothesized that the combined use of Spline and Wavelet would outperform their individual use on data with complex profiles of motion artifacts. To demonstrate this, we first compared, on infant semisimulated data, the performance of several motion correction techniques on their own and of the novel combined approach; then, we investigated the performance of Spline and Wavelet alone and in combination on real cognitive data from three datasets collected with infants of different ages (5, 7 and 10 months), with different tasks (auditory/visual and tactile) and with different NIRS systems. To quantitatively estimate and compare the efficacy of these techniques, we adopted four metrics: haemodynamic response recovery error, within-subject standard deviation, between-subjects standard deviation and number of trials that survived each correction method. Our results demonstrated that (i) it is always better correcting for motion artifacts than rejecting the corrupted trials; (ii) Wavelet filtering on its own and in combination with Spline interpolation seems to be the most effective approach in reducing the between- and the within-subject standard deviations. Importantly, the combination of Spline and Wavelet was the approach providing the best performance in semi-simulation both at low and high levels of noise, also recovering most of the trials affected by motion artifacts across all datasets, a crucial result when working with infant data.
\end{abstract}




\section{Introduction}

Functional near-infrared spectroscopy (fNIRS) is a neuroimaging technique that has experienced an exponential increase in its application to study the infant brain and cognitive development (for a review see Wilcox \& Biondi, 2015). fNIRS has been adopted in several developmental areas, such as social cognition, language, memory, numerosity (for a recent review see Aslin, Shukla, \& Emberson, 2015) and it also shows potential for the study of connectivity (e.g., Bulgarelli et al., 2018; Homae et al., 2010; Molavi et al., 2014). fNIRS infers localized brain activation by quantifying changes in blood oxy-hemoglobin (HbO) and deoxy-hemoglobin (HbR) from changes in intensity of near-infrared light, migrating from a source to a detector (Obrig \& Villringer, 2003). In developmental research, fNIRS is praised because it measures non-invasively brain activity similarly to fMRI, without some of the limitations of the latter (e.g., allowing the study of awake infants in more naturalistic environments). Whereas in fMRI even small head movements can have a large negative impact on data quality, fNIRS, with a good design and tight fit of the headgear, will allow participants a much wider range of movement. This does not mean that the technique is insensitive to motion artifacts; on the contrary, the presence of significant motion artifacts in infant fNIRS time recordings usually constitutes a considerable challenge for the data analysis and recovery of brain activation. Motion artifacts are typically seen in the form of abrupt signal changes and can be classified based on their amplitude and frequency (e.g., spikes, slow drifts, baseline shifts). Despite the improvements achieved in ANIRS technology and headgear sets (Lloyd-Fox, Blasi, \& Elwell, 2010), fNIRS users are still facing the issue of how to best approach motion artifacts that are particularly disrupting in infant fNIRS data.

The presence of motion artifacts in data recordings might affect the shape and validity of the haemodynamic response function (HRF). Indeed, abrupt changes in the signal might mask or inflate the true haemodynamic response, possibly causing, at the level of statistical inference, false negatives or false positives. Furthermore, the undesired modulation of the NIRS signal due to artifacts, as well as improper preprocessing pipelines, might contribute to the variability of haemodynamic responses often reported 
in infant studies (for further discussion on this issue see Issard \& Gervain, 2018). Hence, addressing the issue of motion artifacts is crucial to draw valid neuroscientific conclusions when interpreting fNIRS results and identifying the most appropriate pipeline for motion correction might help reduce the reported variability in infant HRFs and to establish a typical infant haemodynamic response.

In the standard fNIRS processing pipeline, the main options for dealing with motion artifacts are: (i) rejection of the trials contaminated by artifacts; or (ii) correction of the corrupted signal. Trial rejection is not desirable and is often not possible in studies involving challenging populations (such as infants and clinical populations), where the total number of trials per session is often limited by the tolerance and compliance of the participants. Therefore, excluding trials will significantly undermine the calculation of a reliable haemodynamic response and/or weaken statistical power. Thus, in these cases motion correction is needed to reduce the number of rejected trials and, ultimately, the number of excluded datasets from a study. Homer2 (Huppert et al., 2009), is an opensource software for fNIRS data preprocessing that offers a wide range of motion correction methods and it is being used by an increasing number of research groups (e.g., Lloyd-Fox, Széplaki-Köllod, Yin, \& Csibra, 2015; Miguel, Lisboa, Gonçalves, \& Sampaio, 2017; Ravicz, Perdue, Westerlund, Vanderwert, \& Nelson, 2015; Timeo, Brigadoi, \& Farroni, 2017) who are moving away from their in-house fNIRS data processing software options, and are increasingly using motion correction tools over trial rejection.

To date, several motion correction methods have been published and their performances have been compared in previous studies using both simulated and real data (Brigadoi et al., 2014; Cooper et al., 2012). These studies have highlighted that (i) correction is always better than trial rejection; (ii) Spline interpolation (Scholkmann, Spichtig, Muehlemann, \& Wolf, 2010) and Wavelet filtering (Molavi \& Dumont, 2012) are the most effective methods in terms of recovering the haemodynamic responses affected by motion artifacts (Brigadoi et al., 2014; Cooper et al., 2012). It is worth noting 
that all these studies used adult data, both to validate the motion correction technique and to compare the performance of a set of correction techniques.

However, motion artifacts in infant data (and in some clinical adult populations) occur more frequently, sometimes sequentially, and encompass a wider amplitude range compared to what is seen in typical adult datasets. In adult experiments, movement is usually kept to a minimum by simply giving specific instructions to the participants. Therefore, in adult datasets, artifacts are relatively rare and easy to identify. On the contrary, infants cannot be instructed to remain still and, apart from limited cases, motion artifacts typically affect the entire recording. For example, movements occur during bouts of fussiness, boredom or even excitement, which can result in the recording of multiple motion artifacts within a short time window; the variability and the unpredictability of these behaviors, coupled with their high frequency of occurrence during the recording, makes it hard to identify their effects and correct the resulting artifacts that corrupt the data.

Since all motion correction techniques have been validated on adult data and given the substantial difference between the rate of motion artifacts typical in infant compared to adult studies, a worthy question is whether these techniques will show the same performance when applied to datasets with frequent and unpredictable motion artifacts.

To our knowledge, only two studies compared motion correction methods on data from youngsters, one with infant participants (Behrendt, Firk, Nelson, \& Perdue, 2018) and another one with children (Hu et al., 2015). Compared to previous work on adults' and simulated data (Brigadoi et al., 2014; Cooper et al., 2012; Hu et al., 2015), these studies introduce a new approach to motion correction that involves the combined use of two correction techniques previously tested and used separately. This choice is driven by the variability of motion artifacts typically seen in data from young participants: combining two techniques that target different types of motion artifacts should outperform the use of each technique on its own. Specifically, Behrendt and colleagues (2018), compared the performance of Wavelet filtering, targeted Principal Component Analysis (tPCA) and their combination on infant data; their findings indicated that 
Wavelet performs best across all the datasets ( 4 datasets), task types (video vs. live stimuli presentation) and age groups involved (five, seven and twelve-month-olds with $\mathrm{N}=20$ in each age group, six-to-eight-month-olds with $\mathrm{N}=10$ ). Moreover, $\mathrm{Hu}$ and colleagues (2015), demonstrated that for their sample and task (six-to-twelve-year-olds with $\mathrm{N}=12$; language event-related design), the combined use of Wavelet and Moving Average (MA) techniques was preferable to the use of each method on its own, or to other methods (e.g., Spline interpolation, PCA, correlation based signal improvement - CBSI). Although both works, in line with findings on adults' data, confirmed that Wavelet filtering performs well on more noisy data, they did not show a clear advantage in using a combination of motion correction techniques on this type of data. Indeed, the combination of Wavelet with tPCA did not outperform Wavelet alone (Behrendt et al., 2018). Further, it should be noted that the MA method used by $\mathrm{Hu}$ and colleagues (2015) serves the function of a high pass filter (removes slow drifts in the data) and for this reason it cannot be considered a motion correction method such as Wavelet or tPCA. Slow drifts removal is a processing step commonly implemented also in works that discard trials affected by artifacts (e.g., Grossman et al., 2008; Lloyd Fox et al., 2009).

While the approach of combining more than one motion correction technique holds great potential for dealing with infants' data, it is possible that the optimal combination(s) has not yet been identified. Given the consistently efficient performance of Wavelet across age-groups, it is sensible, moving forward, to test new combinations that include this method. With the present work we aim to take a step in this direction and assess the performance of a still untested combination of motion correction techniques: Wavelet filtering and Spline interpolation. The selection of these two methods was guided by previous recommendations from work on adult, children and simulated data (Brigadoi et al., 2014; Cooper et al., 2012; Hu et al., 2015) that compared most of the correction techniques available in Homer2.

Besides introducing this new combination, this work also tests and compares the independent performance of a wide range of motion correction techniques currently available in Homer2 (and of the proposed combination) on infants' semi-simulated 
data: Spline interpolation, Wavelet filtering, tPCA, Wavelet Kurtosis, and Spline Savitzky-Golay.

In brief, the present work has a two-fold aim: 1) test and compare the independent performance of several motion correction techniques on their own and of a novel combination (Spline + Wavelet) on infants' semi-simulated data; 2) investigate the performance of Spline, Wavelet, and their combinations on three different infant datasets. These datasets were collected at different research labs, with different tasks, age-groups, headgears and NIRS acquisition systems. Regarding the analysis on the semi-simulated data we hypothesize that Wavelet and tPCA will perform similarly (Behrendt et al., 2018) and that the new combination will potentially outperform techniques applied individually. If this hypothesis is verified, we also expect that the improved performance of this new combination of motion correction techniques will be greater in signals containing a higher percentage of motion artifacts. Since Wavelet Kurtosis (Chiarelli, Maclin, Fabiani, \& Gratton, 2015) and Spline Savitzky-Golay (Jahani, Setarehdan, Boas, \& Yücel, 2018) are more recent techniques that have never been investigated in a comparative work, a-priori hypotheses cannot be advanced on their performance. With regard to the second part of this work, we hypothesize that the efficacy of each correction method will be highly dependent on the amount and nature of motion artifacts, which in turn might be related to task design, age group, and scalpoptode coupling (that could also be associated with the headgear design) and we expect that the new combination will provide improved or at least similar performance than when applying each technique alone.

The ultimate goal of the present study is to contribute to the common aim of the standardization of infant data preprocessing. The results of this work will provide guidelines for the developmental community for the analysis of infant fNIRS data. 


\section{Materials and Methods}

\section{Infant fNIRS datasets}

In this work, we analyzed data collected in four, independent and unrelated studies, each study contributing one dataset. For convenience, we refer to each dataset using numbers.

\section{Dataset 1}

Data from twelve 11-month-old infants (7 girls, $M_{\text {age }}=347.42, S D_{\text {age }}=10.84$, range $=$ 361-331 days) was retrospectively selected from a group of infants recruited for a longitudinal study at the Centre for Brain and Cognitive Development (CBCD), Birkbeck, University of London (UK) with a resting state protocol while participants were awake. The data was selected from the first time point of the study at 11 months, as it was the closest to the other datasets. Data from seven additional participants was rejected for this analysis because the recording was not long enough for the purpose of this part of the study or there was excessive noise for any of the motion correction techniques to recover any trials (and therefore we would only be measuring noise and not motion correction performance).

The fNIRS data was acquired with the NTS diffuse optical imaging system (Gowerlabs Ltd UK; Everdell et al., 2005). The infants wore a custom-made headgear set consisting of three source-detector arrays located over temporal and frontal regions. 12 source- and 12 detector-optodes were embedded in a custom-built fNIRS headgear, with source-detector separations of $2.5 \mathrm{~cm}$ over the temporal regions and $3 \mathrm{~cm}$ on the frontal regions.

During data acquisition, the infants sat on their parent's lap in a dimly lit and sound-proofed room in front of a 46 -inch plasma screen showing a screensaver video with colored bubbles accompanied by relaxing music, with no identifiable shapes or social stimuli.

Dataset 1 was used to create semi-simulated data, in order to test the performance of the motion correction techniques alone (i.e., Spline, Wavelet, tPCA, Wavelet Kurtosis, and Spline Savitzky-Golay) and the combinations of Spline and Wavelet on a 
dataset with a known haemodynamic response. Three different semi-simulated datasets were created starting from the same resting state data, by adding different types of haemodynamic responses. These three sets of data (see Figure 2.6) were designed to simulate a wide range of data typically collected in infant studies using different stimuli presentation lengths: (1) a $20 \mathrm{~s} \mathrm{HRF,} \mathrm{corresponding} \mathrm{to} \mathrm{a} \mathrm{stimuli} \mathrm{presentation} \mathrm{of} \mathrm{about}$ $10 \mathrm{~s}$ (hereafter referred as "Standard HRF"); (2) a $40 \mathrm{~s}$ HRF, to simulate longer stimuli presentation times ("Block design HRF" in the text); (3) a $12 \mathrm{~s} \mathrm{HRF,} \mathrm{simulating} \mathrm{shorter}$ stimuli presentation that is typical of also event-related designs ("Short HRF" in the text). Haemodynamic responses were simulated by a linear combination of two gammavariant functions (Abdelnour \& Huppert, 2009), the parameters of which were tuned so as to allow small variations in peak amplitude and latency between trials. For the "Standard HRF" dataset, this led to a peak HRF amplitude of $1.43 \pm 0.03 \mu \mathrm{M}$ for $\mathrm{HbO}$ and $-0.68 \pm 0.01 \mu \mathrm{M}$ for HbR; for the "Block Design HRF" dataset, to a peak HRF amplitude of $1.30 \pm 0.01 \mu \mathrm{M}$ for $\mathrm{HbO}$ and $-0.65 \pm 0.005 \mu \mathrm{M}$ for $\mathrm{HbR}$ and for the "Short HRF” dataset, to a peak HRF amplitude of $1.53 \pm 0.05 \mu \mathrm{M}$ for $\mathrm{HbO}$ and $-0.59 \pm 0.02 \mu \mathrm{M}$ for $\mathrm{HbR}$. A minimum of 5 and a maximum of $8 \mathrm{HRF}$ per participant were added in the "Standard HRF" dataset, a minimum of 3 and a maximum of 6 in the "Block Design HRF" dataset and a minimum of 7 and a maximum of 8 in the "Short HRF" dataset, with a variable inter-trial interval (ITI), always long enough to allow the HRF to come back to baseline. ITIs were selected randomly from a normal distribution with mean $(8+\alpha) s$ and standard deviation 2 s. The a value was the length of the simulated HRF in that dataset.

\section{Dataset 2}

Sixteen 4- to 6-month-old infants (6 girls, $M_{\text {age }}=153.81, S D_{\text {age }}=25.67$, range $=115-205$ days) were retrospectively selected from a group of infants who participated in a fNIRS study at the Centre for Brain and Cognitive Behaviour (CBCD), Birkbeck, University of London (UK) (Lloyd-Fox et al., 2018). The aim of the original study was to examine early brain responses to social and non-social stimuli in two groups of infants, one with increased familial risk for later development of Autism Spectrum Disorder (ASD) and 
the other one not. The total number of participants included in the original study was 36 , and, for the present study, we selected the 16 infants included in the low-risk group (i.e., infants with no increased risk for later ASD). A further 9 infants participated in the study but were excluded because they did not attend to enough trials. We chose to include only the low risk group to avoid any bias in the interpretation of the results (see paragraph Metrics of Comparison). Recruitment, ethical approval (UK National Health Service National Research Ethics Service London REC 08/H0718/76 and 06/MRE02/73) and informed consent, as well as background data on participating families with highand low-risk infants, were made available for the ASD study through the BASIS network (http://www.basisnetwork.org). All methods and experimental protocols were approved and carried out in accordance with the NHS and Birkbeck, University of London Ethics Committee guidelines and regulations. Informed consent was obtained from the parent/legal guardian for each participant.

Infants wore custom-built fNIRS headgear consisting of two source-detector arrays, containing a total of 26 channels (source-detector separations: $2 \mathrm{~cm}$; see Figure 2.1a), and were tested with the NTS diffuse optical imaging system (Gowerlabs Ltd UK; Everdell et al., 2005), the same instrument used for collection of Dataset 1 with a $10 \mathrm{~Hz}$ sampling rate. This system used two continuous wavelengths of source light at 770 and $850 \mathrm{~nm}$. The two fNIRS arrays were placed bilaterally on the participants' head and covered from the inferior frontal to the posterior temporal regions (Lloyd-Fox et al., 2018). During data acquisition, infants sat on their parent's lap facing a 46-inch plasma screen situated about $100 \mathrm{~cm}$ away where visual stimuli were displayed. Hidden behind the screen, two speakers played auditory stimuli. The experimental condition trials, displayed for 9-12 s each, alternated one after the other, with a reference trial (silent presentation of visual static non-social images, also displayed for 9-12 s) between each. During the three types of experimental conditions (silent, S; auditory vocal, V; auditory non-vocal, N) the infants were looking at videos displaying a social interaction. In between two consecutive experimental conditions, static images of vehicles were displayed with no sounds. The conditions were presented in the same order across 
infants until the infants became bored or fussy as judged by the experimenter who was monitoring their behavior (see Lloyd-Fox et al., 2018 for more details).

\section{Dataset 3}

Seventeen 5-month-olds ( 9 girls; $M_{\text {age }}=163.4, S D_{\text {age }}=12.73$, range $=127-182$ days $)$ took part in the experiment conducted at Utrecht University, The Netherlands; two additional infants were excluded because they viewed less than three trials per condition. Both parents gave informed consent prior to participation. The Medical Ethical Committee of the University Medical Centre of Utrecht approved the study (protocol number: NL50617.041.14, METC 14-526), which was conducted in accordance with the Declaration of Helsinki.

The fNIRS data was acquired with the NTS diffuse optical imaging half-system (NTS2 - Gowerlabs Ltd UK; Everdell et al., 2005), consisting of eight dual-wavelength sources (780 nm, $850 \mathrm{~nm}$ ) and eight detectors, which form 22 source-detector channels at a separation of $2 \mathrm{~cm}$ (see Figure 2.1b). Data were sampled at a frequency of $10 \mathrm{~Hz}$. The probe array was placed over the infants' right hemisphere, covering parts of the occipital, temporal and frontal cortices.

During the experiment infants sat on their parent's lap at $\sim 60 \mathrm{~cm}$ from a 23 -inch computer monitor (refresh rate $60 \mathrm{~Hz}, 1920 \times 1080$ resolution), in a dimly lit room. The task consisted in alternating 5-second experimental trials displaying sequences of five female pictures posing either happy or fearful expressions interleaved with $\sim 10 \mathrm{~s}$ baseline trials displaying sequences of at least 10 houses (the end of the baseline trial was controlled by the experimenter). Every stimulus was displayed for $800 \mathrm{~ms}$ and followed by a $200 \mathrm{~ms}$ inter-stimulus interval showing a fixation cross; the order of the stimuli was randomized within trials (for more details see Chapter 4, i.e. Di Lorenzo et al., 2019).

\section{Dataset 4}

Twenty-two 10 -month-old infants (13 girls; $M_{\text {age }}=331.74, S D_{\text {age }}=22.88$, range $=281-$ 370 days) took part in the experiment; eight additional infants took part in the experiment but were excluded from the study owing to fussiness. This experiment was carried out at Keio University, Tokyo, Japan. Parents gave informed consent in 
compliance with a protocol approved by the ethic committee of Keio University, faculty of letters (14034-0-2).

The fNIRS data was acquired with the Hitachi ETG-7000 (Hitachi, Tokyo, Japan), consisting of eight dual-wavelength sources $(780 \mathrm{~nm}, 830 \mathrm{~nm})$ and seven detectors, which formed 22 source-detector channels at a separation of $2 \mathrm{~cm}$ (see Figure 2.1c). Data were sampled at a frequency of $10 \mathrm{~Hz}$. The probe array was placed over the infants' right hemisphere, covering parts of the parietal, temporal and frontal cortices.

During the task infants sat on their parents' lap in front of a table. An experimenter sat next to the infant and delivered brushstrokes on the infant's right forearm at two different speeds: $3 \mathrm{~cm} / \mathrm{s}$ or $30 \mathrm{~cm} / \mathrm{s}$. A second experimenter sat in front of the infant and distracted him/her with toys to prevent the infant from directing his attention to the tactile stimulation. Each experimental stimulus lasted 10 seconds and was followed by a baseline trial, where no touch was applied, with a duration of either 10 or 15 seconds. The order of the stimuli was fully randomized.

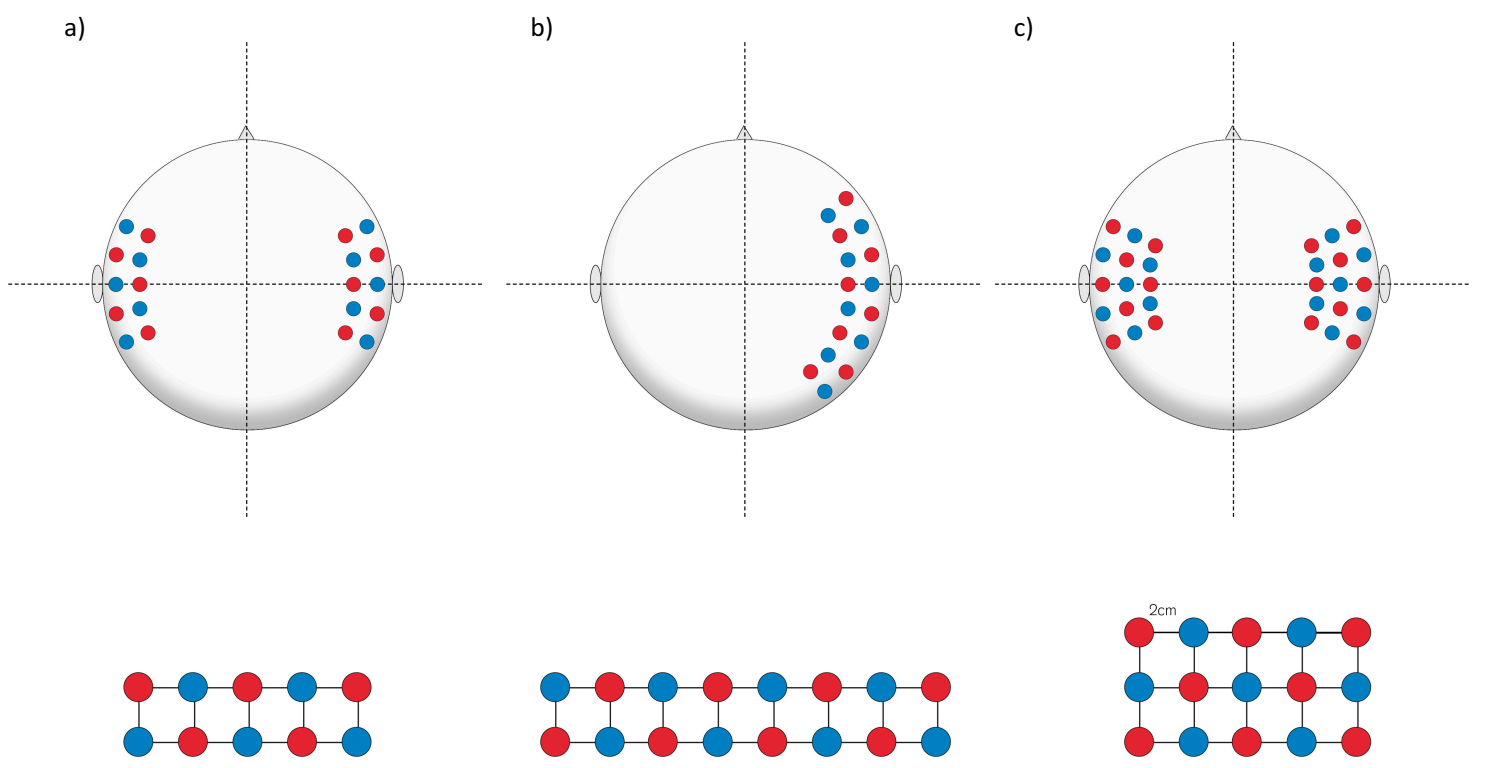

Figure 2.1 Probe layout and locations of the channels for a) Dataset 2, b) Dataset 3, and c) Dataset 4. Red dots represent sources and blue dots indicate detectors.

\section{Dataset comparison: contamination by motion artifacts}

Since infant data are likely to be contaminated more than adult data by motion artifacts, we expected different performance of the motion correction techniques depending on 
the amount of artifacts. Therefore, as first step, we evaluated the degree of motion artifact contamination in each dataset. To this end, we quantified for each channel and subject of each of the four datasets the percentage of signal identified as motion artifact (prior to correction) relative to the total duration of the signal. Motion artifactual sections of the signal were identified as described in the section below called Trial Rejection.

\section{Motion correction techniques ${ }^{1}$}

The main goal of this work is to investigate on different infant datasets and acquisition systems whether the combination of Spline and Wavelet overcomes the use of these techniques alone and of other techniques currently available in Homer2. We included the two possible combinations of Spline and Wavelet to examine whether the sequential order of application of the functions influenced their performance (hereafter referred to as $\mathrm{Spl}+\mathrm{Wav}$, with the opposite-ordered combination referred as $\mathrm{Wav}+\mathrm{Spl})$. In particular, we tested on Dataset 1 (semi-simulated data with a known haemodynamic response) the performance of the following methods: trial rejection, Spline, Wavelet, Spl+Wav, Wav+Spl, tPCA, Wavelet Kurtosis, and Spline Savitzky-Golay. As Wavelet requires the user to set specific tuning parameters, we further investigated on Dataset 1 whether different parameters can influence the performance of this motion correction technique. Based on the results of Dataset 1, we chose to evaluate the performance on real data of trial rejection, Spline, Wavelet, Spl+Wav, Wav+Spl, which were tested using Datasets 2, 3, and 4 .

\section{Trial Rejection}

Trial Rejection consists in the exclusion of trials contaminated by motion artifacts from further analysis. The excluded trials were pre-selected in a channel-by-channel mode by the Homer2 function hmrMotionArtifactByChannel. This function detects the signal

\footnotetext{
${ }^{1}$ All analyses presented in this work were run using Homer2. To aid Homer2 users, in the main text we always report the Homer 2 abbreviations for functions and parameters. However, it is important to note that the same functions can be implemented in processing streams outside of the Homer2 environment, where different naming conventions are used.
} 
exceeding a threshold in change of amplitude (AMPthresh) or/and a threshold in change of standard deviation (STDEVthresh) within a predefined time-window (tMotion) and marks as artifacts the data points around the detected motion (+/-tMask). After the detection of artifacts, a second step rejects the corrupted trials from all channels (not on a channel-base). All parameters for this function are defined by the user. For each dataset, we selected values that identified the majority of spike-like motion artifacts (see Table 2.1). In particular, values of standard deviation thresholds were decided after visual inspection of each dataset in order to optimize motion detection and avoid both the over-identification of signal as noise and to miss important artifacts. This same approach was used in Cooper et al. (2012).

Table 2.1 Parameters used in hmrMotionArtifactByChannel and hmrMotionArtifact functions for each dataset.

\begin{tabular}{l|llll}
\multicolumn{1}{c}{} & Dataset 1 & Dataset 2 & Dataset 3 & Dataset 4 \\
\hline tMotion & 1 & 1 & 1 & 1 \\
tMask & 1 & 1 & 1 & 1 \\
STDEVthresh & 13 & 15 & 15.5 & 13.5 \\
AMPthresh & 0.4 & 0.4 & 0.4 & 0.4
\end{tabular}

\section{Spline}

Spline interpolation, first proposed by Scholkmann and colleagues (2010), is a channelby-channel correction method that acts on previously detected motion artifacts (hmrMotionArtifactByChannel; see description in the section on Trial Rejection). After motion detection, the spline function corrects the artifact by performing a cubic spline interpolation of the artifact; the interpolation is then subtracted from the original signal. After this, the signal is baseline corrected to ensure that signal time-course before and after the corrected artifact is continuous. Spline interpolation depends on a parameter (p) that can be set by the user; in this study we used $p=0.99$, the same value used by previous studies (Brigadoi et al., 2014; Cooper et al., 2012; Scholkmann et al., 2010). The positive aspect of this correction method is that it only corrects the pre-localized artifacts without modifying the other portions of the time-series. However, it depends heavily on 
the ability of the motion detection step in identifying motion artifacts and hence on the parameters set in the motion detection function (hmrMotionArtifactByChannel).

\section{Wavelet}

The wavelet filtering available in Homer2, first proposed by Molavi and Dumont (2012), is a function that detects and corrects artifacts channel-by-channel in a single step. This function decomposes the signal time-course of every channel in a series of wavelet detail coefficients which are characterized by a Gaussian distribution: while the coefficients linked to the physiological components (NIRS signal of interest) will be distributed around zero, the coefficients reflecting motion artifacts can be identified as the outliers of the Gaussian distribution. Then, by setting to zero all detail coefficients identified as outliers of the distribution (< first quartile - $a$ times the interquartile range or $>$ third quartile $+a$ times the interquartile range) and reconstructing the signal with the modified coefficients (with the inverse discrete wavelet transform), we can obtain a version of the original signal with a much reduced presence of motion artifacts. In Homer2 the a threshold can be defined by setting the tuning parameter iqr. The advantage of the wavelet method is that it does not require a prior step of motion artifact detection, however the choice of iqr value will depend on the timing of the task paradigm and consequently on the characteristics of the evoked HRF. For instance, using a too low iqr will reduce and/or filter out the haemodynamic response itself in event-related designs. For Dataset 1, six different iqr values were employed (iqr = 0.1, 0.5, 0.8, 1.0, 1.2, and 1.5) to evaluate the influence of the choice of iqr on motion correction and HRF estimation. For Datasets 2, 3 and 4 we used $i q r=0.8$, which was defined by visually inspecting the effects of this and the other iqr values on the group-averaged HRFs (i.e., $1.2,1.0,0.5,0.1)$; also, this value yielded a good performance in recovering the haemodynamic response and the number of trials when tested on semi-simulated data. 


\section{tPCA}

Targeted Principal Component Analysis (tPCA) was first described by Yücel and colleagues (2014). This motion correction method, like Spline interpolation, acts on previously detected motion artifacts (using the function hmrMotionArtifactByChannel) and is applied to artifactual parts of the signal time-course across all channels. First motion artifacts are detected. Then, segments of data containing motion artifacts are extracted from the original signal of all channels and concatenated together into a new single data matrix. The correction technique (PCA) is applied only to this dataset which mainly consists of epochs of motion ('targeted'). PCA applies an orthogonal transformation to this dataset composed of $\mathrm{N}$ measurements (number of channels) to produce $\mathrm{N}$ uncorrelated components, ordered by their contribution to the variance of the data. Thus, the first components will account for the largest proportion of the variance of the data. Since motion artifacts should constitute a large proportion of the variance of the data, the first $M$ components should represent the variance caused by the motion artifacts. Removing the first $\mathrm{M}$ components from the signal should result in the correction of the motion artifacts (Zhang, Brooks, Franceschini, \& Boas, 2005). The corrected segments are stitched back into the original signal. Analogously to Spline, the signal is baseline corrected to ensure that the time-course before and after the corrected artifact is continuous. This procedure can be reiterated for a number of times (as defined by the user) to allow for correction of any residual motion artifacts not corrected during the first iteration.

In Homer2, tPCA depends on two parameters that can be set by the user: the percentage of variance (nSV) and the maximum number of iterations (maxiter). In this study, we used $\mathrm{nSV}=0.97$, that is we removed $97 \%$ of the total variance, and maxIter $=$ 5, the same values suggested by Yücel and colleagues (2014). The performance of PCA is directly dependent on the number of measurements available, i.e., on the number of channels. To test this dependency, we run tPCA on Dataset 1 twice, once by using the complete dataset and once by using only the channels made up by half of the available sources and half of the available detectors, to emulate a smaller dataset. 


\section{Wavelet Kurtosis}

Kurtosis-based Wavelet filtering (Wav Kurt) was first proposed by Chiarelli and colleagues (2015) with the aim to overcome some of the limitations associated with wavelet filtering (i.e. in cases of high signal-to-noise ratio (SNR) this correction can lead to the reduction of signal amplitude). The novel idea introduced by the authors is that the wavelet coefficients generated from fNIRS signals have sub-Gaussian (kurtosis < 3) or Gaussian (kurtosis $=3$ ) distributions. In contrast, data affected by motion artifacts tend to have larger kurtosis values, reflecting the presence of outliers. Unlike the a value of wavelet filtering, the kurtosis value is independent of the SNR of the data.

Analogously to wavelet filtering, kurtosis-based wavelet filtering performs a wavelet transformation of the data and computes the distribution of the wavelet coefficients. The presence of motion artifacts is assessed by computing the kurtosis of this distribution and evaluating whether it is higher than a given threshold. If this is the case, the most extreme coefficients are set to zero, the kurtosis computed again and compared to the given threshold. This procedure iterates until the computed kurtosis is smaller than the given threshold. After this, the inverse wavelet transformation is applied and the signal reconstructed. In this work, we used a kurtosis threshold of 3.3, as suggested in the original paper (Chiarelli et al., 2015).

\section{Spline Savitzky-Golay}

The combination of Spline interpolation with Savitzky-Golay (SG) filtering was recently proposed by Jahani and colleagues (2018). The authors intended to devise a single approach that could deal with different types of motion artifacts. They proposed the combination of Spline interpolation, which best corrects baseline shifts, followed by SG filtering, a method suited for correction of high frequency spikes. This algorithm, as shown in the original paper, works when the SNR of the data is greater than 3, while the authors suggest to apply only the SG filtering part of the algorithm when $\mathrm{SNR}<3$. In this work, for the Spline part of the algorithm we employed the same parameters defined in the sections above on Trial Rejection and Spline. For the SG part of the algorithm, we set the frame size to $6 \mathrm{~s}$, as in the original work (Jahani et al., 2018). 


\section{Data Processing streams and values used in each dataset}

Data was processed using the Homer2 package in MATLAB. We created different processing streams, each tailored for a specific correction method: trial rejection, Spline, Wavelet, Spl+Wav, Wav+Spl, tPCA, Wavelet Kurtosis, and Spline Savitsky-Golay (Figure 2.2 reports the steps of all processing streams). We also processed the datasets without correcting for motion artifacts (No Correction approach).

a) Processing stream Datasets $1,2,3,4$

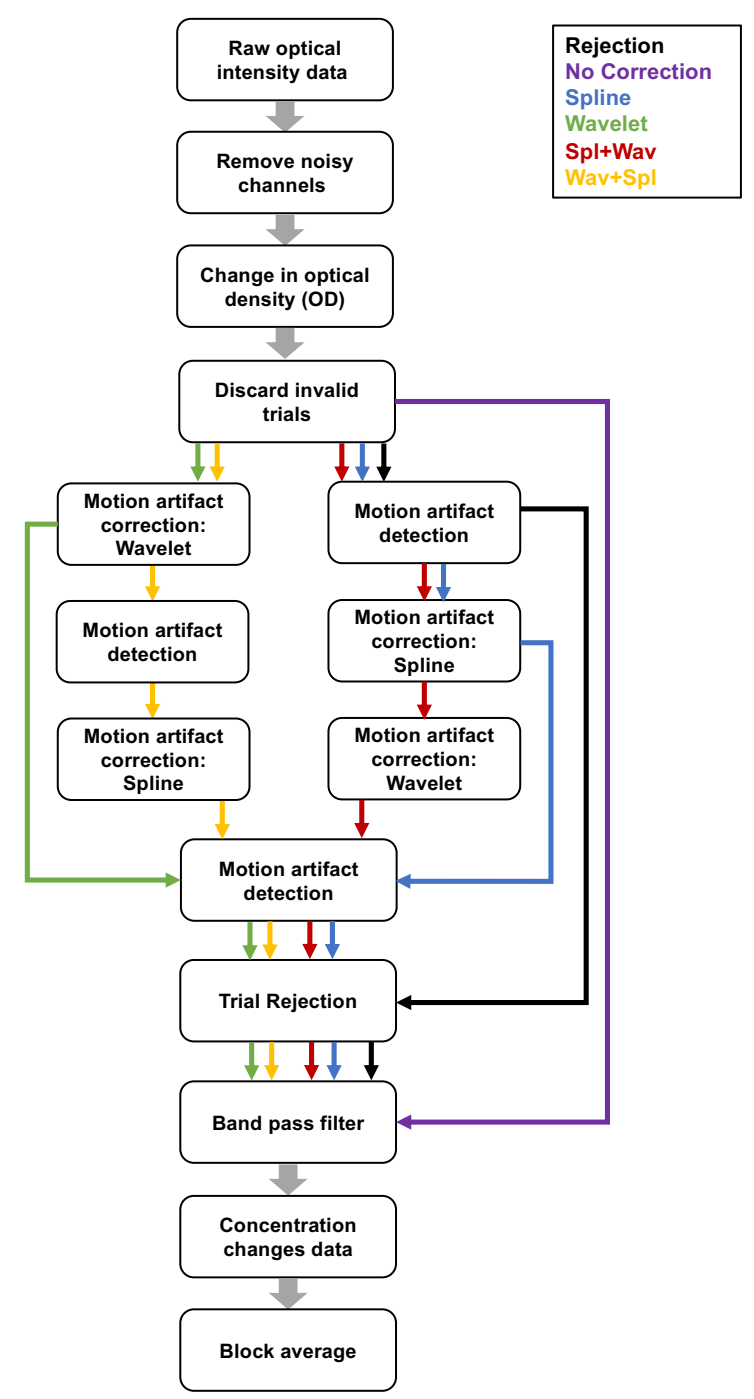

b) Processing stream for Datasets 1 only

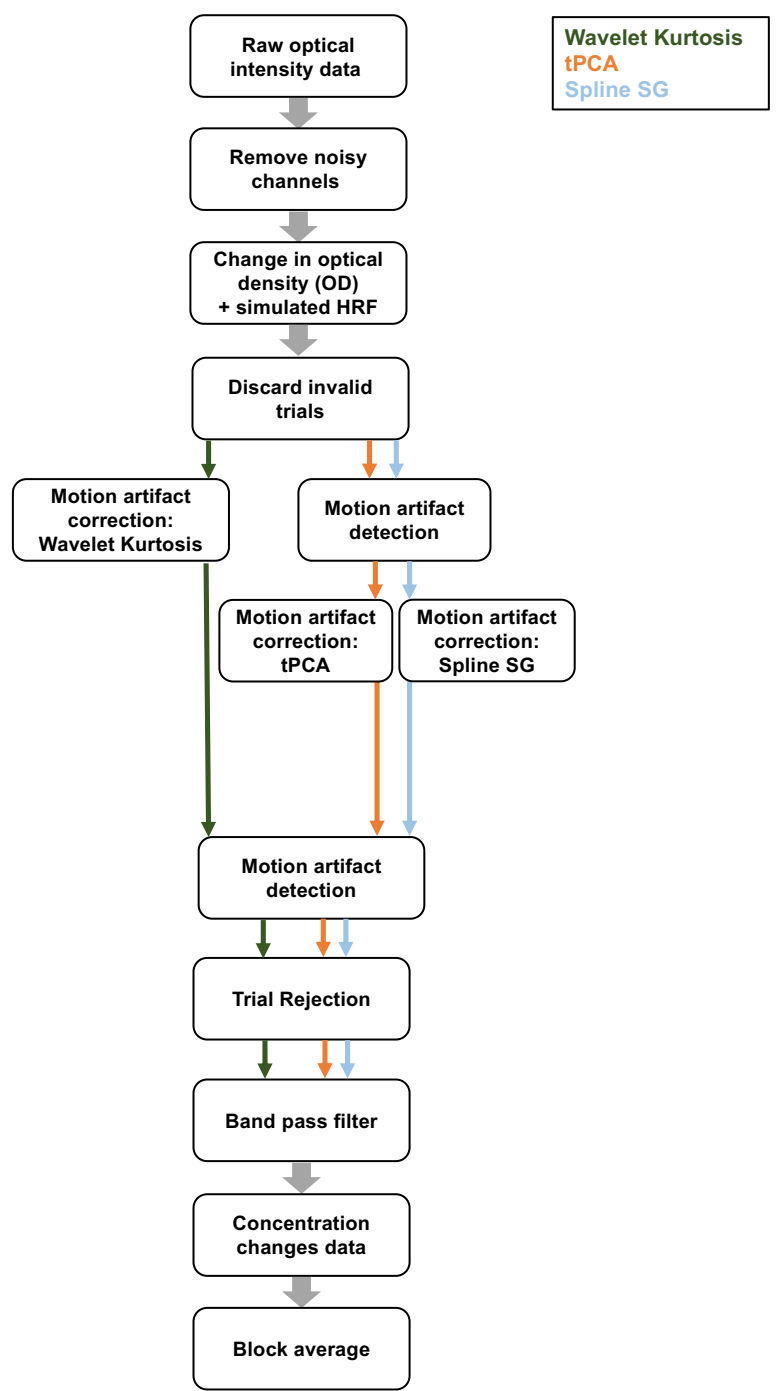

Figure 2.2 Processing streams of the techniques applied to all datasets (a) and to Dataset 1 only (b). Each coloured arrow represents the specific workflow for each technique, while thicker grey arrows indicate the common processing steps. 
For each processing stream, channels showing very high or low optical intensity readings were excluded from further analyses (using the function enPruneChannels). Intensity thresholds were differently chosen for each device according to the company recommendations. After that, the raw intensity data were converted to optical density (OD) changes. Then invalid trials (e.g., non-looking trials) were discarded. From this step on, different streams were applied (see Figure 2.2). After any correction method, motion detection, using hmrMotionArtifact, was applied to identify the remaining uncorrected motion artifacts; the parameters chosen for this function were the same as the ones used in hmrMotionArtifactByChannel (see Table 2.1). Note that motion detection was also part of the trial rejection stream. A subsequent step allowed the rejection of trials affected by motion artifacts, for all processing streams but the No Correction one. After this step, a band-pass filter (third order Butterworth) was applied to reduce slow drifts and high-frequency noise. Note that specific values for the cut-off frequencies of the band-pass filter were set for each dataset to maximize the reduction of noise without removing or corrupting the haemodynamic responses, taking into account the different stimulation timing of each experiment (e.g., Dataset 3, having the shortest stimuli presentation time allowed the use of a higher frequency for the low cutoff). Then the OD data were converted to concentration changes using the modified Beer-Lambert law (Cope \& Delpy, 1988; Delpy et al., 1988) with differential pathlength factor of 5.1 (Duncan et al., 1995). Finally, all remaining trials were block-averaged for every condition, channel and participant. Table 2.2 reports the values of the parameters of the functions used in the processing streams used for each dataset. 
Chapter 2

Table 2.2 Parameters and values used in each processing stream for each of the four datasets. Abbreviations for functions and parameters used in Homer2 are in italics.

\begin{tabular}{|c|c|c|c|c|c|c|c|}
\hline & & \multicolumn{3}{|c|}{ Dataset 1} & \multirow[t]{2}{*}{ Dataset 2} & \multirow[t]{2}{*}{ Dataset 3} & \multirow[t]{2}{*}{ Dataset 4} \\
\hline & & $\begin{array}{c}\text { Standard } \\
\text { HRF }\end{array}$ & $\begin{array}{c}\text { Block } \\
\text { Design } \\
\text { HRF }\end{array}$ & $\begin{array}{l}\text { Short } \\
\text { HRF }\end{array}$ & & & \\
\hline \multirow{4}{*}{$\begin{array}{l}\text { Channel rejection } \\
\text { enPruneChannels }\end{array}$} & \multirow{2}{*}{$\begin{array}{l}\text { Intensity } \\
\text { range } \\
(\text { dRange })\end{array}$} & $1.00 \mathrm{E}-03$ & $1.00 \mathrm{E}-03$ & $1.00 \mathrm{E}-03$ & $1.00 \mathrm{E}-03$ & $2.00 \mathrm{E}-03$ & $9.00 \mathrm{E}-01$ \\
\hline & & $1.00 \mathrm{E}+07$ & $1.00 \mathrm{E}+07$ & $1.00 \mathrm{E}+07$ & $1.00 \mathrm{E}+07$ & $1.00 \mathrm{E}+07$ & $4.00 \mathrm{E}+05$ \\
\hline & $\begin{array}{c}\text { SNR } \\
\text { threshold }\end{array}$ & 0 & 0 & 0 & 0 & 0 & 0 \\
\hline & $\begin{array}{l}\text { Source- } \\
\text { Detector } \\
\text { separation } \\
\text { range } \\
\text { (SDrange) } \\
\end{array}$ & 0.045 .0 & 0.045 .0 & 0.045 .0 & 0.045 .0 & 0.045 .0 & 0.045 .0 \\
\hline $\begin{array}{l}\text { Spline } \\
\text { interpolation } \\
\text { hmrMotionCorrect } \\
\text { Spline } \\
\end{array}$ & $p$ & 0.99 & 0.99 & 0.99 & 0.99 & 0.99 & 0.99 \\
\hline $\begin{array}{l}\text { Wavelet filtering } \\
\text { hmrMotionCorrect } \\
\text { Wavelet }\end{array}$ & $i q r$ & $\begin{array}{c}0.1 / 0.5 / \\
0.8 / 1.0 / \\
1.2 / 1.5\end{array}$ & $\begin{array}{c}0.1 / 0.5 / \\
0.8 / 1.0 / \\
1.2 / 1.5\end{array}$ & $\begin{array}{l}0.1 / 0.5 / \\
0.8 / 1.0 / \\
1.2 / 1.5\end{array}$ & 0.8 & 0.8 & 0.8 \\
\hline \multirow{2}{*}{$\begin{array}{l}\text { Targeted PCA } \\
\text { hmrMotionCorrect } \\
\text { PCArecurse }\end{array}$} & $\begin{array}{l}\text { Variance } \\
\text { amount \% } \\
(n S V)\end{array}$ & 0.97 & 0.97 & 0.97 & - & - & - \\
\hline & $\begin{array}{l}\text { Max \# of } \\
\text { iterations } \\
\text { (maxIter) } \\
\end{array}$ & 5 & 5 & 5 & - & - & - \\
\hline $\begin{array}{l}\text { Wavelet Kurtosis } \\
\text { hmrMotionCorrect } \\
\text { KurtosisWavelet }\end{array}$ & $\begin{array}{l}\text { Kurtosis } \\
\text { threshold } \\
\text { (kurt) }\end{array}$ & 3.3 & 3.3 & 3.3 & - & - & - \\
\hline \multirow{2}{*}{$\begin{array}{l}\text { Spline Savitzky- } \\
\text { Golay } \\
\text { hmrMotionCorrect } \\
\text { SplineSG }\end{array}$} & $p$ & 0.99 & 0.99 & 0.99 & - & - & - \\
\hline & $\begin{array}{c}\text { FrameSize_ } \\
\text { sec }\end{array}$ & 6 & 6 & 6 & - & - & - \\
\hline $\begin{array}{l}\text { Trial rejection } \\
\text { based on artifact } \\
\text { presence } \\
\text { enstimRejection }\end{array}$ & $\begin{array}{l}\text { time Range } \\
\text { (tRange) }\end{array}$ & -2.010 .0 & -2.010 .0 & -2.010 .0 & -2.010 .0 & -2.05 .0 & -2.010 .0 \\
\hline \multirow{2}{*}{$\begin{array}{l}\text { Bandpass filter } \\
\text { hmrBandPass }\end{array}$} & hpf & 0.01 & 0.01 & 0.01 & 0.01 & 0.03 & 0.025 \\
\hline & $\operatorname{lpf}$ & 1 & 1 & 1 & 1 & 0.8 & 1 \\
\hline $\begin{array}{l}\text { Block average } \\
\text { hmrBlockAverage }\end{array}$ & $\begin{array}{c}\text { time Range } \\
(\text { tRange })\end{array}$ & -2.020 .0 & -2.040 .0 & -2.012 .0 & -2.020 .0 & -2.015 .0 & -2.020 .0 \\
\hline
\end{tabular}




\section{Metrics of Comparison}

In order to compare the performance of the motion correction techniques, we used four metrics: the haemodynamic response recovery error, measured with the root mean squared error (RMSE) (applied on Dataset 1), the within-subject standard deviation (applied on Datasets 2, 3,4), the between-subjects standard deviation (applied on Datasets 2, 3, 4), and the number of trials that survived each correction method (applied on all datasets). As for the number of trials, the within-, and between-subjects SD, every motion correction technique was first compared to the No Correction approach (purple path in Figure 2.2), which does not correct or reject trials affected by motion artifacts, and then to each other. Both results for $\mathrm{HbO}$ and $\mathrm{HbR}$ concentration changes were investigated.

For the semi-simulated Dataset 1, two types of comparisons were performed: the first investigated the impact of changing the iqr value in the Wavelet motion correction technique, while the second investigated the performance of all motion correction techniques in semi-simulation. Therefore, for the first comparison, the performance of the Wavelet technique was compared across iqr values (i.e., $0.1,0.5,0.8,1.0,1.2,1.5$ ), whereas when comparing performance across techniques (and the Wavelet-Spline combinations), only two options for the Wavelet parameter iqr were tested: $i q r=0.5$ and iqr $=0.8$. These two iqr values were chosen because they showed the highest performance in the recovery of the HRF and of the number of trials on the first comparison. Note that an iqr of 1.0 also performed well in these two metrics, but with a higher variability. In addition, the infant literature reports studies using iqr values of 0.5 (e.g., Ravicz et al., 2015). Therefore, we decided to further test only the two lower iqrs, 0.5 and 0.8 .

\section{Haemodynamic response recovery error}

The RMSE between the true simulated HRF and the HRF recovered by each processing stream was computed for each of the three sets of data of Dataset 1, each participant and channel. The true HRF was computed as the average of the true single-trial HRFs for 
each participant and channel. Results are reported aggregated across channels. A lower RMSE is an index of improved HRF estimate.

\section{Within-subject standard deviation}

The within-subject standard deviation (SD) compares, for every participant, the mean of the standard deviation of the single-trial haemodynamic responses across channels and conditions. The assumption behind this metric is that most of the variability between single-trial haemodynamic responses within the same participant should be due to the presence of motion artifacts.

\section{Between-subjects standard deviation}

The between-subjects SD was used to investigate the variability between the averaged haemodynamic responses across subjects for every channel and condition. Similarly to the within-subject SD, we assume that most of the variability between haemodynamic responses across subjects is due to the presence of motion artifacts.

\section{Number of recovered trials}

We also quantified the number of trials included in the averaged HRFs for every participant and condition, after the different correction methods were applied. The number of trials recovered by each technique for each participant was normalized on the original number of trials available in each participant (i.e. on the number of trials obtained with the No Correction approach).

\section{Statistical analyses}

Statistical analyses were performed using SPSS (IBM Corporation, Armonk, NY, USA).

The mean of the percentages of motion artifacts calculated across channels for each subject and wavelength was submitted to a one way ANOVA with dataset as betweensubject factor, to evaluate differences in motion artifact contamination between datasets; independent samples t-tests were used to follow-up the significant difference among the datasets.

To compare the performance of the techniques, separate repeated measure ANOVAs with technique as within-subject factor were computed for the number of 
recovered trials (for all datasets), the RMSE (for Dataset 1) and the within-subject SD (for datasets 2, 3 and 4) metrics for both chromophores (HbO, HbR). For the RMSE and the within-subject SD, we calculated for every subject a unique value representative of each technique, which consisted in the mean of all values across channels and conditions. Main effects of technique were followed up with two-tailed paired sample ttests to compare the performances of all the techniques to each other, within each dataset. The False Discovery Rate (FDR) criteria was employed to correct for multiple comparisons (note that all figures display FDR-corrected results).

\section{Motion correction performance at different percentages of artifact contamination}

A further analysis was performed on the semi-simulated data of Dataset 1 to evaluate the motion correction performance (indexed by the RMSE) at different percentages of motion artifact contamination. This analysis was devised to understand whether the different performance of each motion correction technique compared to the other was constant across the different percentages of presence of motion artifacts or diverged as this percentage increased.

For each subject and channel, we computed the percentage of motion artifact contamination as described above in the Dataset comparison section, and for each subject, channel and motion correction method, we used RMSE as metric of its performance. The amount of available data at lower percentages of motion artifact contamination was higher, getting sparser at increased percentages of motion artifacts. Therefore, we fitted a linear model (B-spline with 3 degrees of freedom) on the RMSE values at the different percentages of motion artifacts and we predicted the RMSE values at the missing points, from 0 to $21 \%$ of motion artifact contamination, at steps of $0.5 \%$ (there were only few samples exceeding $21 \%$ of noise, not providing enough power to perform statistical analyses). Within this framework, we evaluated the confidence intervals of the estimate as well. For each pair of motion correction techniques, and at each estimated percentage of motion contamination, a t-test was performed to evaluate whether the performance of the two techniques differed statistically. We employed the Welch's t-test, which takes into account the unequal variance of the two populations. 
The standard deviation of the two populations at each percentage of motion artifact was computed knowing the estimated RMSE value and the confidence interval at that point (90\% confidence level). The degrees of freedom were computed using the WelchSatterthwaite equation. FDR was employed to correct for multiple comparisons (separately for each comparison between any two technique). All these analyses were performed with the RStudio software package (RStudio Team, 2016).

We hypothesize that at very low percentages of motion artifact contamination all techniques will perform similarly. Then, as the number of motion artifacts increases, standard techniques used for motion correction in adult datasets (e.g., Wavelet) should outperform the Rejection, No correction and Spline techniques. Further, as the number of motion artifacts reaches levels compatible with infants datasets, the combination of Spline and Wavelet should outperform the other techniques, thus demonstrating both the importance of evaluating motion correction techniques in infants dataset and that in this type of datasets stacking two techniques could be ideal.

\section{Results}

\section{Motion artifact quantification}

The assessment of the percentage of motion artifacts per channel and participant revealed that Dataset 2 was the least noisy one, with a maximum of $12.5 \%$ of signal identified as motion artifact $(M=2.21, S D=2.72$, range $=0-12.5 \%)$, while the noisiest datasets were Dataset $1(M=7.26, S D=6.76$, range $=0-46.0 \%)$, and Dataset $3(M=6.13$, $S D=7.83$, range $=0-65.7 \%)$; Dataset 4 showed an intermediate level of noise $(M=3.99$, $S D=3.85$, range $=0-18.1 \%)$. Note that the amount of motion artifact contamination in a signal is dependent on the size of the temporal mask employed (tMask).

The one-way ANOVA (with Dataset as between subject factor) showed a significant difference between the percentage of artifacts in each dataset, $F(3,70)=11.05$, $p<.001, \eta_{p}{ }^{2}=.32$. Post-hoc t-tests confirmed what previously described, that is Dataset 2 was less corrupted by motion artifacts than Dataset $1(t(19.6)=-5.98, p<.001)$, Dataset $3(\mathrm{t}(17.1)=-4.14, p=.002)$, and Dataset $4(t(27.6)=-3.83, p=.002)$; whereas a similar 
percentage of artifacts was observed between Datasets 1 and $3(t(34)=0.91, p=.37)$, and Datasets 3 and $4(t(22.7)=2.09, p=.058)$. Also, Dataset 1 had a higher percentage of artifacts compared to Dataset $4(t(27.3)=3.52, p=.003)$.

\section{Motion correction performance in semi-simulation (Dataset 1)}

\section{Tuning parameters of Wavelet}

Figure 2.3 reports the performance of the Wavelet motion correction technique across iqr values for both the normalized number of recovered trials and the RMSE metric. Results for each set of data of Dataset 1 are reported in the three panels.

The number of recovered trials was significantly different among iqr values in both the "Standard HRF" and the "Short HRF" sets of data $(F(5,55)=3.21, p=.013$ and $F(5,55)=3.51, p=.008$, respectively) but not in the "Block Design HRF" set $(F(5,55)=$ $1.59, p=.177)$. In particular, there was a trend towards a higher trial recovery with $i q r=$ 1 compared to $i q r=1.2$ or 1.5 and with $i q r=0.8$ compared to $i q r=1.5$ in both the "Standard HRF" and the "Short HRF" sets of data. Furthermore, there was a trend towards a higher trial recovery with iqr $=0.5$ compared to $i q r=0.1$ in the "Standard HRF" and compared to iqr $=1.5$ in the "Short HRF" set of data $(\min t=2.65, \max p=$ .082 , corrected, $\mathrm{df}=11)$.

RMSE was significantly different among iqr values in the "Standard HRF" set of data $(F(5,55)=3.18, p=.014)$, although no significant differences emerged from the $\mathrm{t}$ tests comparing the different techniques. No significant differences between techniques were found in the "Short HRF" and in the "Block Design HRF" sets of data $(F(5,55)=$ $0.85, p=.520$ and $F(5,55)=0.49, p=.780$, respectively). 

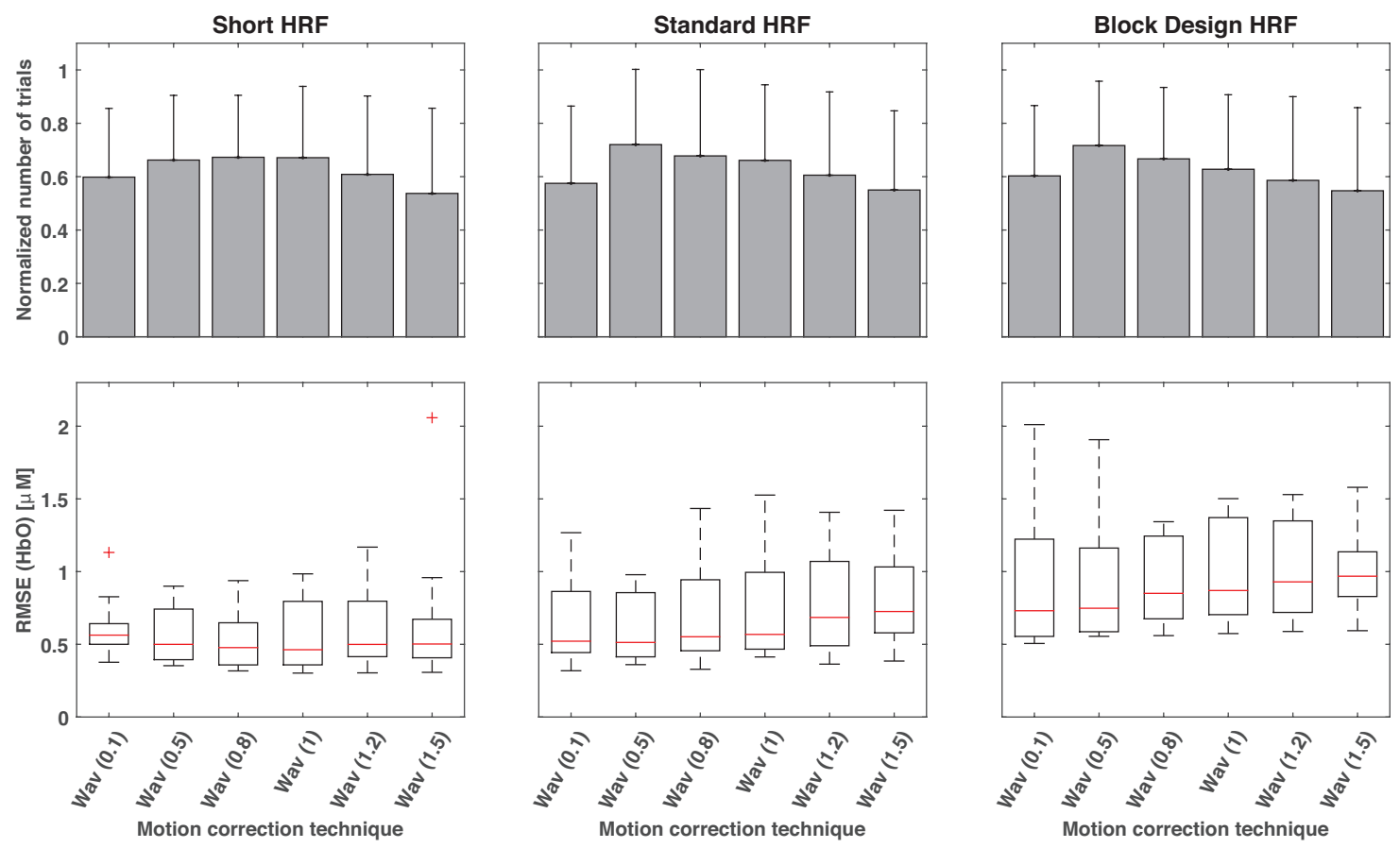

Figure 2.3 Normalized number of trials (upper row) and RMSE (bottom row) recovered after applying the Wavelet motion correction technique with different iqr values for each set of data of Dataset 1. Errorbars in the upper panels represent standard error across participants. The red line in the box plots of the bottom panels depicts the median value, the two whiskers denote the first and third quartile and outliers are represented by red crosses.

\section{Performance of correction techniques in simulated HRFs}

Figure 2.4 reports the number of trials recovered after applying each motion correction processing pipeline normalized to the original number of trials (equivalent to the number obtained with the No Correction approach) for each participant. Results for each set of data of Dataset 1 are reported in the three panels. Note that the results relative to the performance of Spline Savitzky-Golay are not shown in this and the following figures since the application of this technique had a strong impact on the signal, causing the rejection of all the trials in all subjects.

Regarding the remaining correction methods, the number of recovered trials was significantly different among techniques in all three sets of data $(\min F=15.3$, all $p$ s $<$ $.001)$. The results of the $\mathrm{t}$-tests between techniques are reported in Figure 2.4 and are consistent across sets of data. In general, while Rejection and Wavelet Kurtosis are the 
techniques that significantly recover less trials, tPCA and the combinations of Spline and Wavelet (regardless of order and iqr value) are the approaches recovering almost all trials.
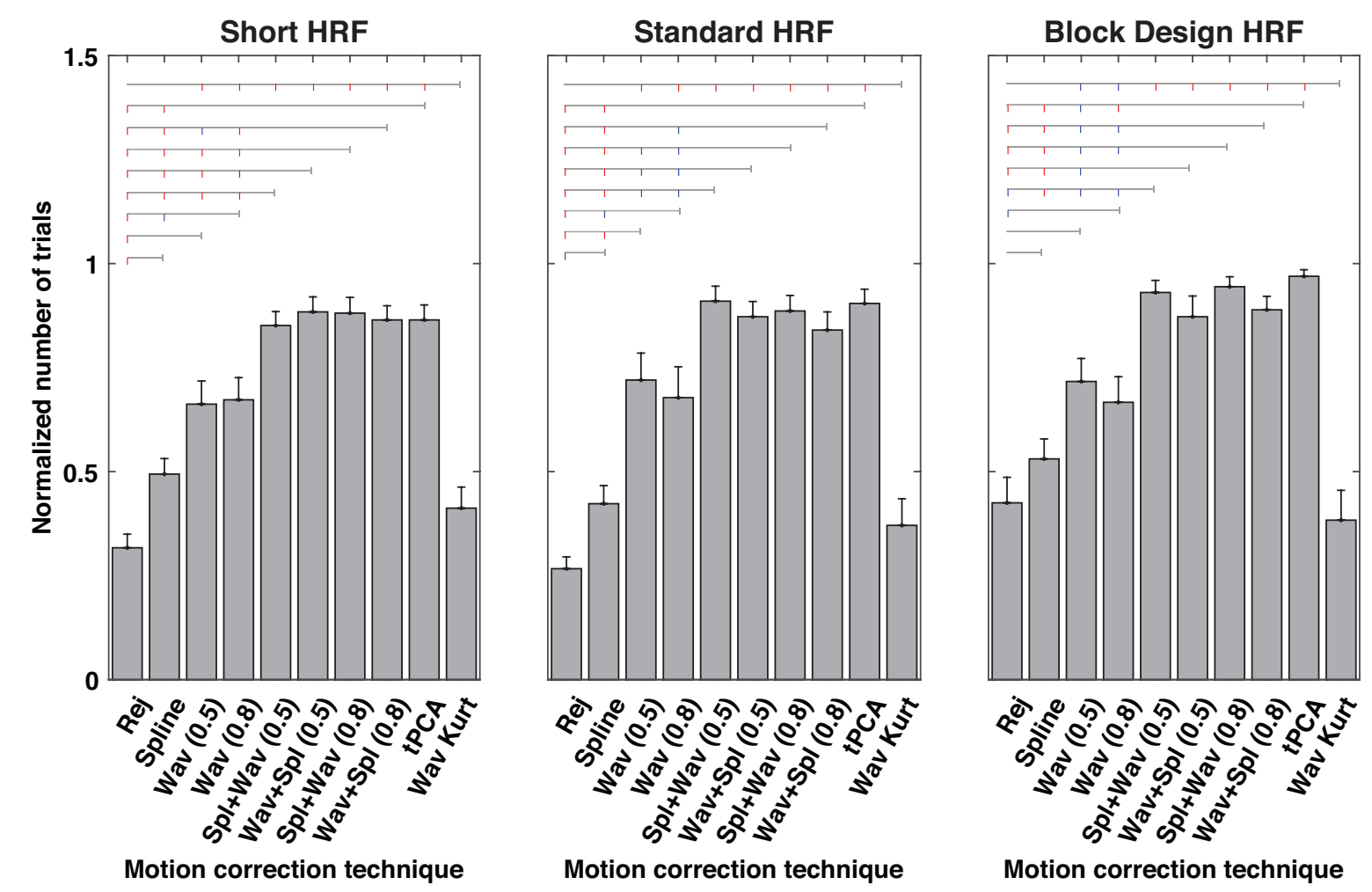

Figure 2.4 Normalized recovered number of trials for each processing pipeline and for each of the three sets of data of Dataset 1. Error-bars indicate standard error across participants. The lines above linking the different techniques indicate significant statistical difference: blue lines correspond to $p<.05$ and red lines to $p<.01$. Degrees of freedom $=11$.

Figure 2.5 reports the RMSE obtained after applying each motion correction processing pipeline (see Figure S2.2 for the HbR results). Results for each set of data of Dataset 1 are reported in the three panels. RMSE was significantly different among techniques in all three sets of data $(\min F=2.03, \max p=.037)$. Pairwise $\mathrm{t}$-tests between techniques are reported in Figure 2.5. For all sets of data, trial rejection, No Correction, and Wavelet Kurtosis perform worse. tPCA and Wavelet showed similar performance levels, with Wavelet scoring a slightly lower median RMSE compared to tPCA, although this is not statistically significant. However, tPCA showed a smaller interquartile range compared to Wavelet. The performances of the combinations of Spline and Wavelet 
were also comparable with those of Wavelet and tPCA, however, the combined approach reported the lowest median RMSE across all HRF types and, for the "Block Design HRF", the haemodynamic response was significantly better recovered by Spl+Wav (0.5) than tPCA.

Further analyses on tPCA, comparing the results of tPCA using all available channels and tPCA using approximately half the number of channels, showed that its performance is dependent on the number of channels available, although this difference was not statistically significant, likely due to the low power of this analysis. We report these analyses in the Supplementary Materials I (section: Analyses on tPCA).

Overall, the method scoring the lowest median RMSE for all sets of data was the combination of Spline (performed at first step) and Wavelet (performed at second step), which therefore proved to be a robust method across different types of haemodynamic responses. Interestingly, in the "Short HRF" set of data, the lowest RMSE was scored by this combination when using iqr $=0.8$, while for the "Standard HRF" and the "Block Design HRF" when using iqr $=0.5$. This result is in line with the formulation of Wavelet approach: lowering the iqr, indeed, sets to 0 more detail coefficients, increasing the chances to reduce or even remove the actual haemodynamic response. Detail coefficients represent the output of the different high-pass filters performed during wavelet decomposition. In the "Short HRF" set of data, the haemodynamic response has higher frequency components than in the "Standard HRF" and in the "Block Design HRF” sets of data. Therefore, it should be hypothesized that it is more likely that some of the detail coefficients contain information related to the haemodynamic response in the "Short HRF" dataset and therefore a higher threshold for rejection should be selected. 

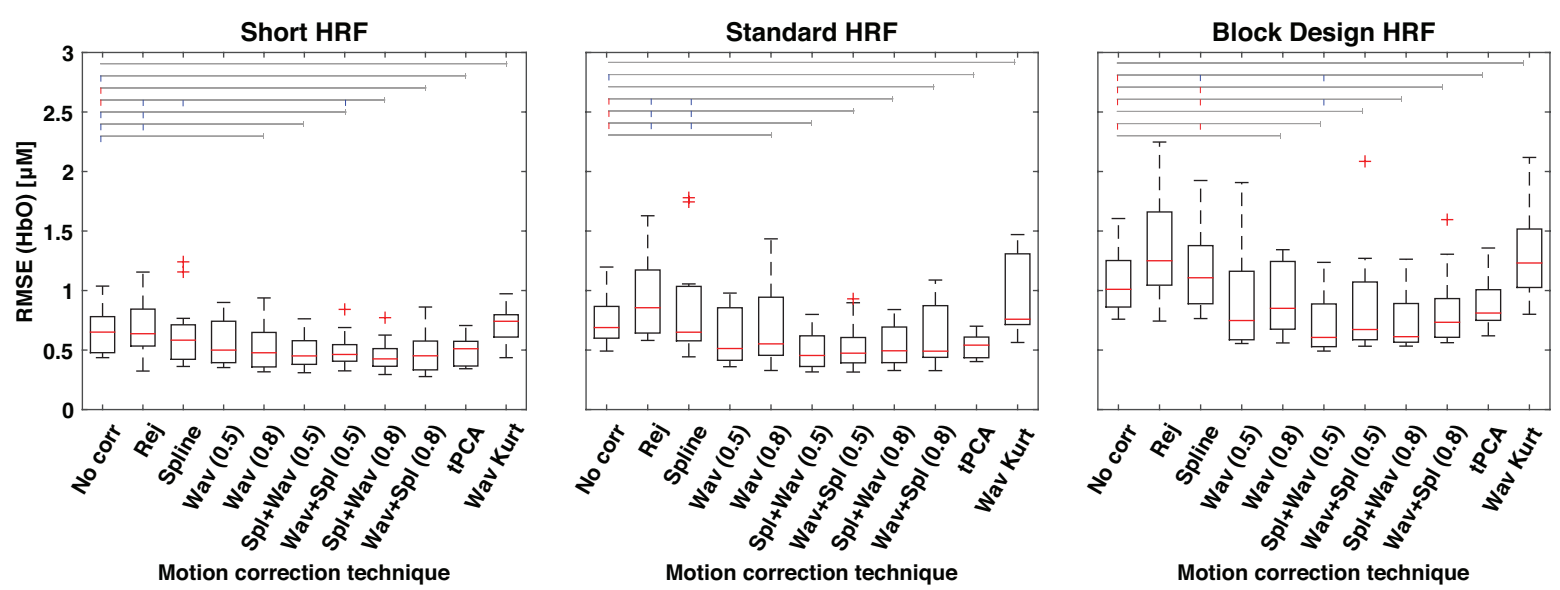

Figure 2.5 RMSE for each processing pipeline and for each of the three sets of data of Dataset 1 . The red line in the box plots depicts the median value, the two whiskers denote the first and third quartile and outliers are represented by red crosses. The lines above linking the different techniques indicate the significant statistical difference: blue lines correspond to $p<.05$ and red lines to $p<.01$. Degrees of freedom $=11$.

Figure 2.6 reports example individual average HRFs for one channel recovered after applying the different motion correction techniques on the three sets of data (see Figure S2.3 for the HbR responses).
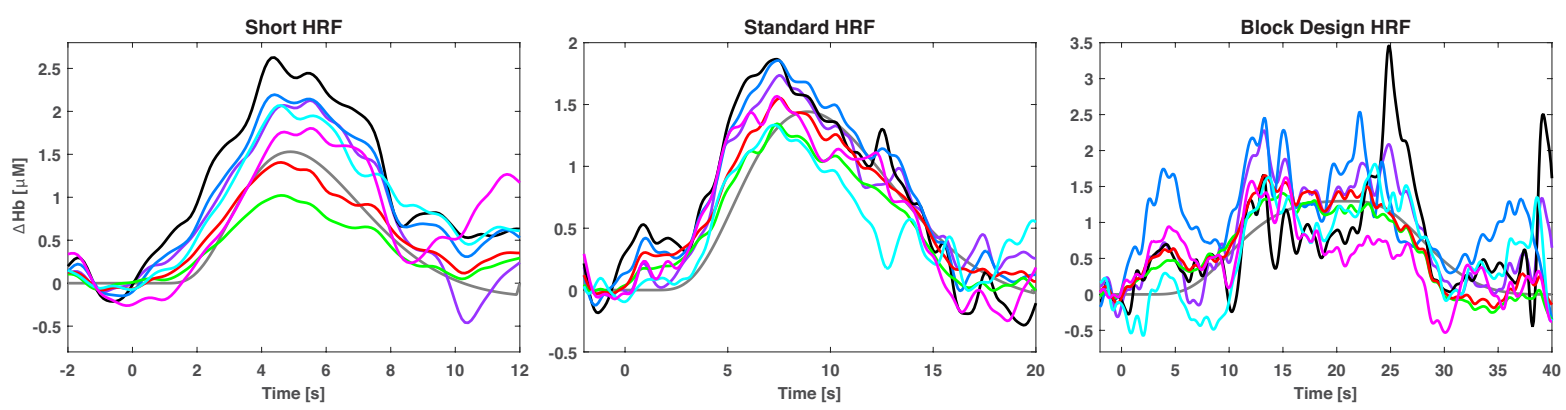

-True - No corr — Rej — Spline — Wav (0.5) — Spl+Wav (0.8) — TPCA — Wav Kurt

Figure 2.6 Examples of individual average $\mathrm{HbO}$ responses for one channel recovered after applying different motion correction approaches on the three sets of data of Dataset 1 . Only some of the tested techniques are reported for visualization purposes. The missing techniques, Wavelet with iqr $=0.8$, Spl+Wav with $i q r=0.5$ and Wav+Spl with both iqrs performed similarly to Spl+Wav (0.8), Wavelet (0.5), Wavelet (0.5) and Spl+Wav (0.8), respectively. The true simulated HRF is displayed in grey. 


\section{Motion correction performance at different percentages of motion artifact contamination}

The performance of almost all motion correction techniques was dependent on the amount of motion artifacts identified in the signal, with a worsening of the performance at increased percentages of motion artifact contamination (see Figure 2.7 reporting $\mathrm{HbO}$ results, and Figure S2.5 for the HbR results). This decline in the performance was smaller for Wavelet, tPCA and above all for the combination of Spline and Wavelet (with Spline applied before Wavelet).

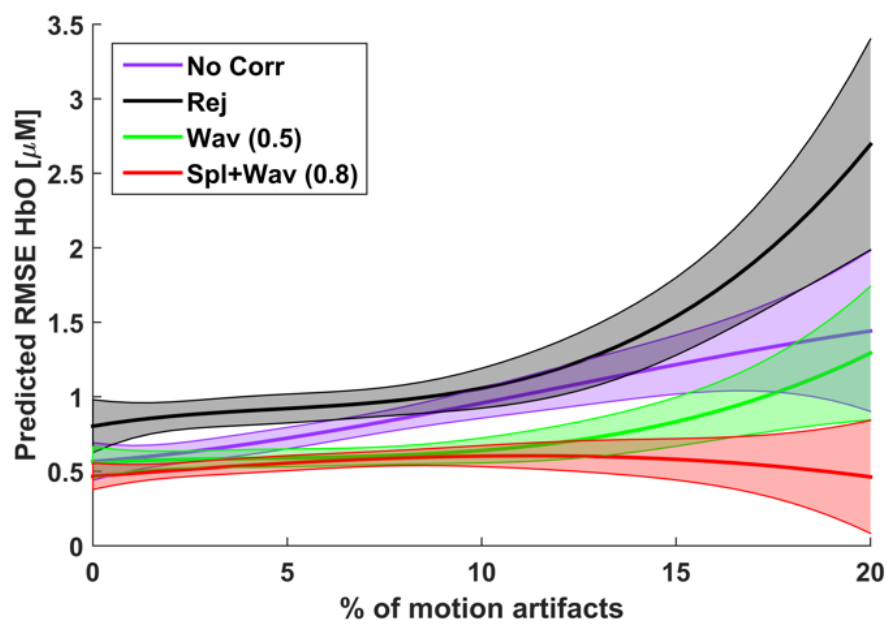

Figure 2.7 Estimated RMSE HbO values at different percentages of motion artifact contamination for a selection of motion correction methods. Rejection, No Correction, Wav (0.5) and Spl+Wav (0.8) were selected for visualization purposes. The shaded areas represent the confidence intervals.

Figure 2.8 displays the corrected p values resulting from the Welch's t-test at three exemplary percentages of motion artifacts $(0 \%, 5 \%$ and $15 \%)$ for each comparison between techniques for $\mathrm{HbO}$ (see Figure S2.6 for the HbR results). As expected, when no motion artifacts were detected only few comparisons resulted statistically significant. At increasing percentages of motion artifact contamination, the performance of the different techniques started to diverge, with Wavelet (at iqr $=0.5$ ), the stacked techniques and tPCA showing statistically significant better performances compared to the other techniques. Note that $0 \%$ corresponds to the percentage of artifacts identified by the detection process rather than the absence of artifacts; it is possible that some subtler artifacts were not captured. 

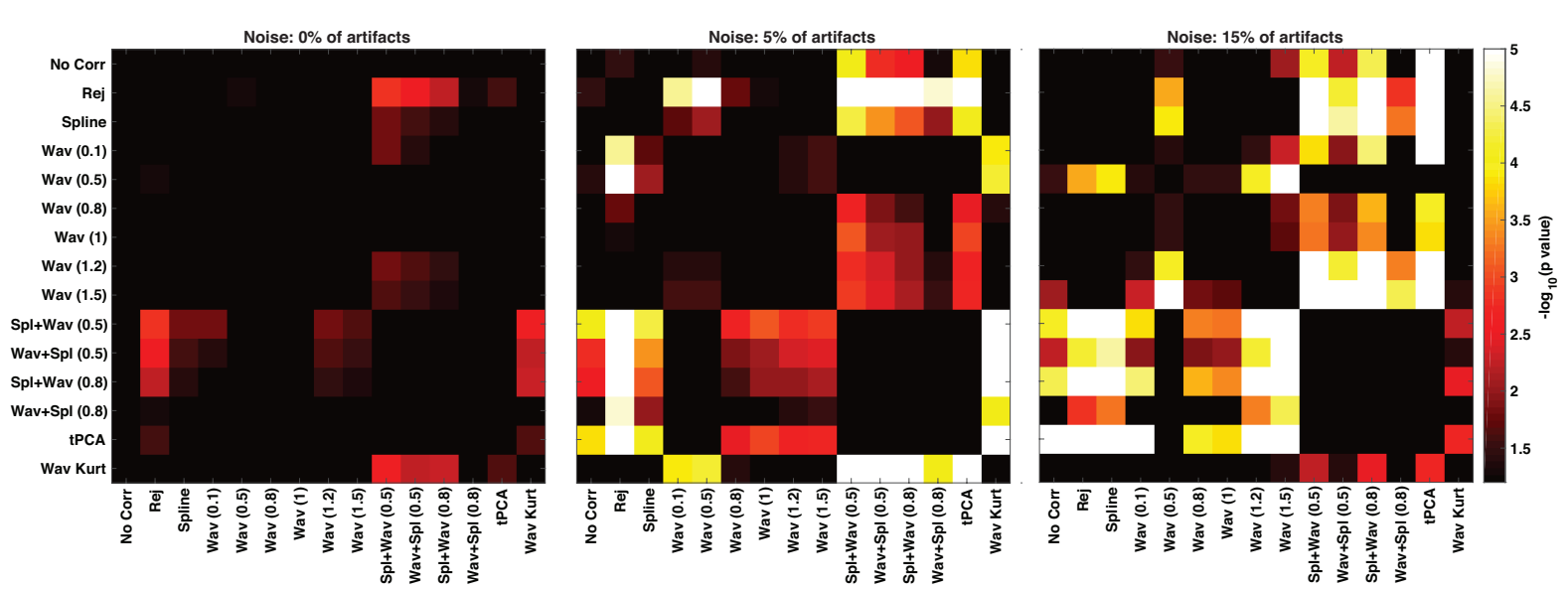

Figure 2.8 Corrected p values resulting from the paired t-tests computed between any pair of correction methods on the RMSE HbO values of the "Standard HRF" dataset at $0 \%, 5 \%$, and $15 \%$ of artifact contamination. For visualization purposes, we selected only 3 levels of noise for one HRF type. The colorbar represents $-\log 10$ (p value) for visualization purposes (the value 1.2, which is the lower limit, corresponds to $p=.05$, higher values correspond to $p<.05$, the higher the more significant).

\section{Motion correction performance in task-based datasets (Datasets 2, 3, 4)}

For reasons of brevity and since the results for $\mathrm{HbO}$ and $\mathrm{HbR}$ were similar, in the main manuscript we report only the $\mathrm{HbO}$ results; $\mathrm{HbR}$ results are reported in the Supplementary Materials I. For each dataset, repeated measures ANOVA analysis of within-subject SDs revealed a main effect of technique for all datasets (all $p s<.0001$ ). In general, across all datasets, No Correction, Rejection and Spline performed poorly in reducing the within-subject SDs, while Wavelet and the two possible combinations of Spline and Wavelet reduced more effectively this measure. However, subtle differences can be observed between datasets. For instance, for datasets 3 and $4 \mathrm{Spl}+\mathrm{Wav}$ was more effective in SD reduction, while for Dataset $2 \mathrm{Wav}+\mathrm{Spl}$ performed best. Figure 2.9 reports the $\mathrm{p}$-values of all the $\mathrm{t}$-tests performed between each technique.

Both combinations of Spline and Wavelet recovered most of the trials affected by artifacts in all datasets (see Figure 2.10). 

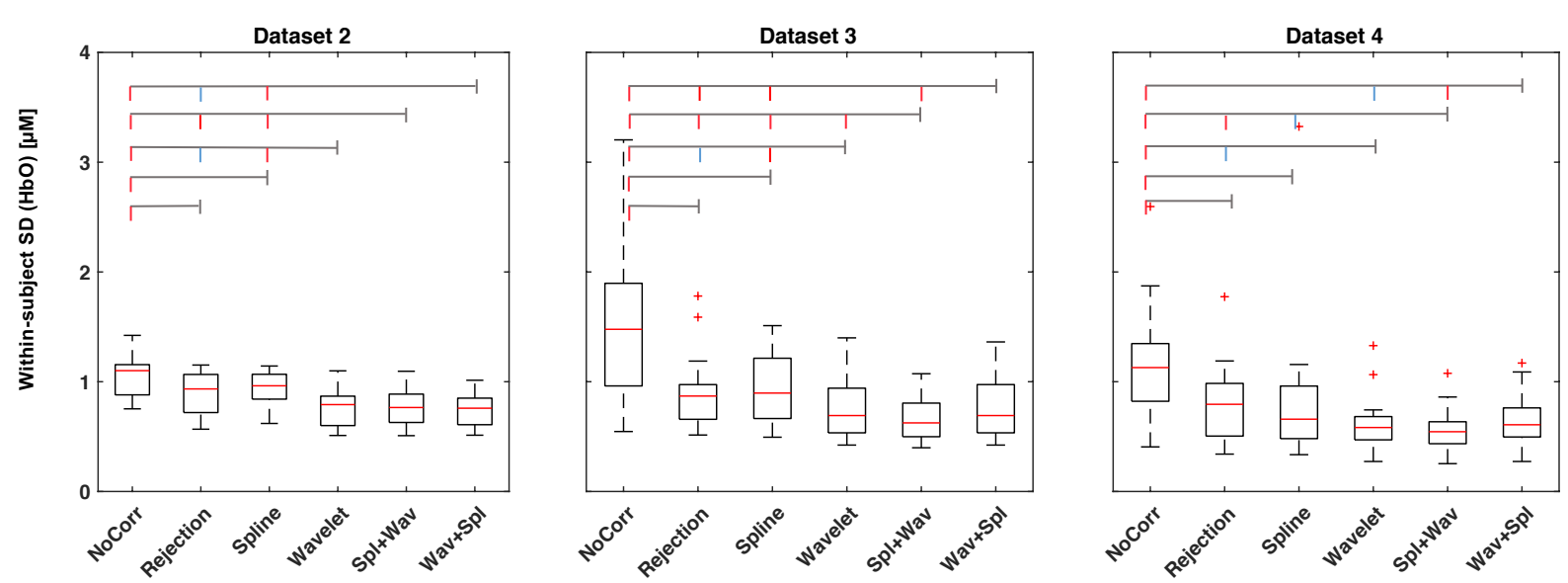

Figure 2.9 Box plots of the within-subject SD calculated for $\mathrm{HbO}$ of all techniques of Datasets 2, 3 and 4. The red line in the box plots indicates the median, while the two whiskers denote the first and third quartile. Outliers are represented with red crosses. The lines above linking the different techniques indicate significant statistical differences: blue lines correspond to $\mathrm{p}<.05$ and red lines to $\mathrm{p}<.01$. Degrees of freedom: Dataset 2, $\min =13, \max =15$; Dataset $3, \min =14, \max =16$; Dataset $4, \min =16$, $\max =21$.

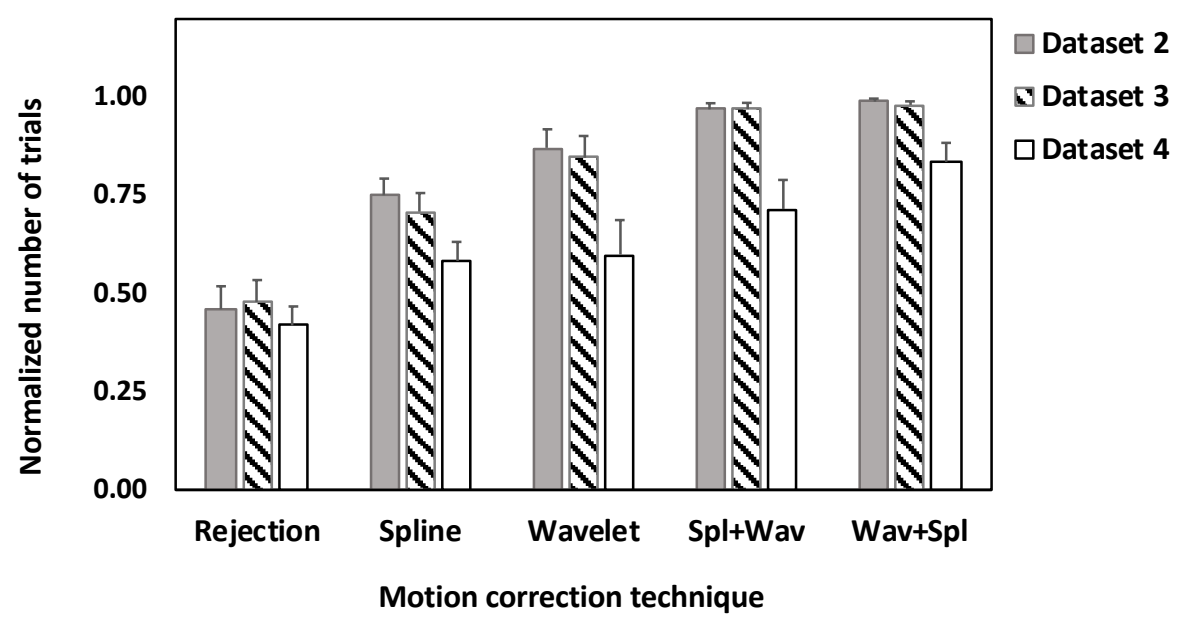

Figure 2.10 The bars represent the mean number of trials averaged for each technique and dataset, normalized to the mean number of trials averaged when no motion correction was applied; the error bars indicate standard error (SEM).

\section{Performance of Rejection versus No Correction}

The between-subjects SD scatter plots (first column of Figure 2.11) show that, for every dataset, rejecting trials performs worse than including noisy trials, increasing the between-subjects SD among the subjects' mean HRF more than $60 \%$ of the time. This 
outcome could be the result of some very noisy mean HRFs measured from infants that had many trials rejected.

Instead, the rejection method has a better performance reducing the within-subject SD as compared to the No Correction approach (second column of Figure 2.11); the rejection of trials affected by motion artifacts is indeed efficient in reducing the variability between trials within each subject. For all datasets, paired t-tests supported this and showed a significant difference between these two techniques (all $p s<.01$; see Figure 2.9). Rejection excluded more than $50 \%$ of the total presented trials in each dataset (Figure 2.10).

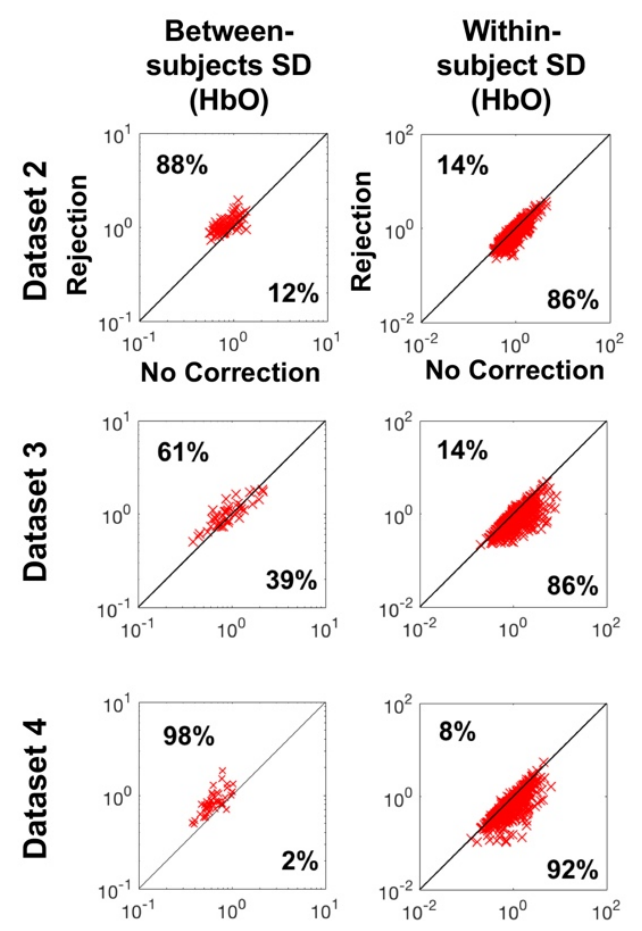

Figure 2.11 Scatter plots of the between-subjects (left column) and within-subject (right column) SD calculated for the comparison between Rejection ( $y$ axis) and No Correction technique (x axis) for HbO values of datasets 2,3 and 4 (row 1,2,3, respectively). The rejection of trials affected by predefined motion artifacts decreases the between-subjects SD between 39\% and 2\% of the time for $\mathrm{HbO}$ compared to No Correction, across all datasets (left panels); however, when looking at a single dataset the percentage of standard deviation reduction has a smaller range (right panels). 


\section{Performance of Motion Correction techniques versus No Correction}

All the correction methods significantly reduced the within-subject SD across all datasets (Figure 2.9; paired t-tests No Correction vs. correction, all ps <.01). Specifically, we found that the Wavelet and its combinations with Spline (Wav+Spl and Spl+Wav) performed best at reducing the SD in $97 \%$ to $100 \%$ of cases (Figure 2.12 lower panel). Spline alone reduced the within-subject SD in 77\% to $90 \%$ of the cases (Figure 2.12 lower panel). Two-tailed paired t-tests between all the correction methods considered in this study revealed that: (i) Wavelet significantly reduced the within-subject SD compared to Spline in Datasets 2 and 3 ( $p s<.001$ ), and to the combination of Wav+Spl in Dataset $4(p<.03)$; (ii) Spl+Wav significantly decreased the SD when compared to Spline in all datasets $(p s<.03)$, to Wavelet $(p s<.04)$ and Wav+Spl $(p s<.002)$ in Datasets 3 and 4; (iii) Wav+Spl significantly reduced the SD when compared to Spline in Datasets 2 and $3(p s<.007)$ and to Spl+Wav in Dataset $2(p<.01)$.

The between-subjects SD was reduced in $100 \%$ of cases with Wav+Spl in all datasets, with Spl+Wav performing similarly (Figure 2.12 upper panel). These techniques on their own have a lower power in reducing the between-subjects SD, with Spline having the worst performance. Note that all techniques succeeded in reducing the between-subjects SD metric relative to the No Correction approach. 


\section{Between-subjects SD Correction HbO}
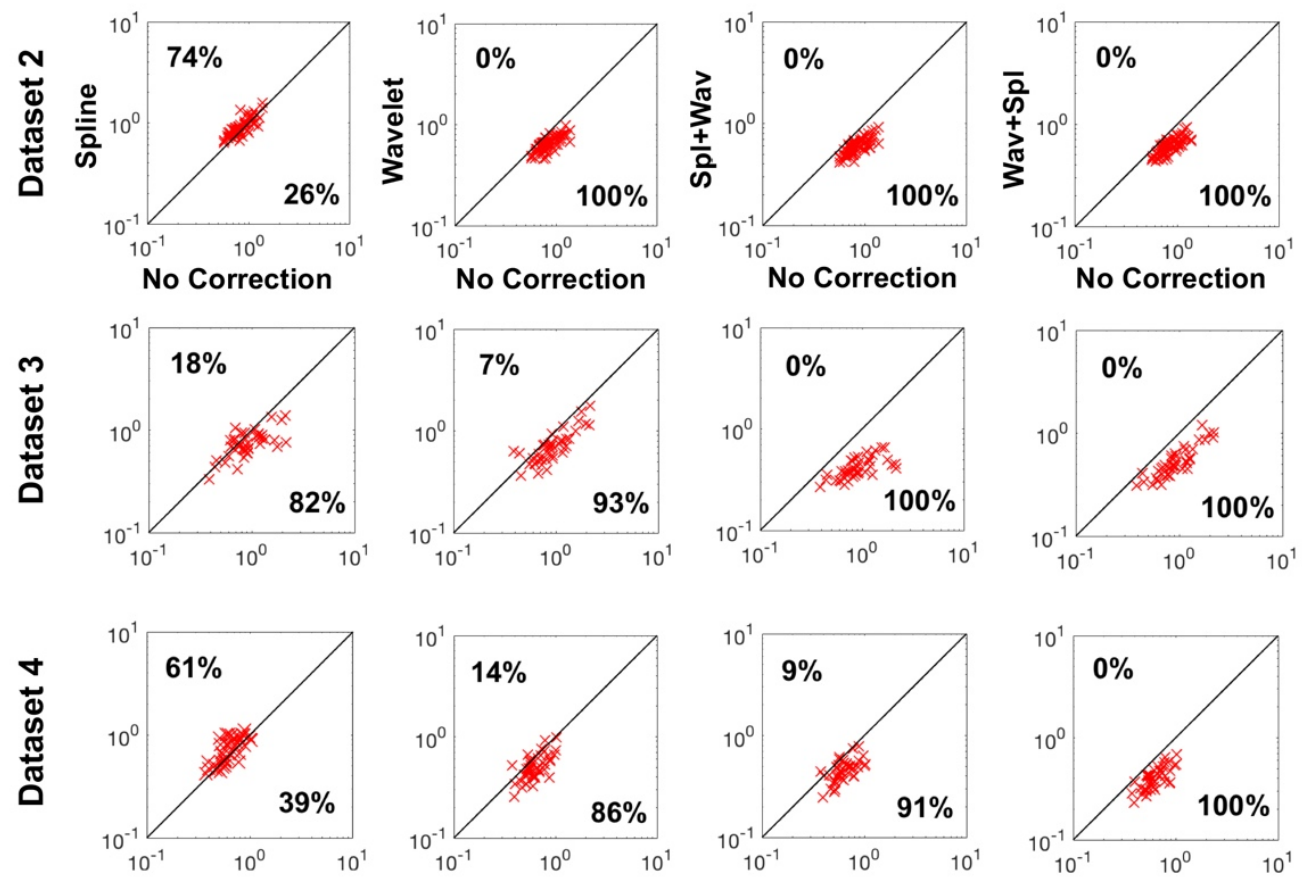

Within-subject SD Correction HbO
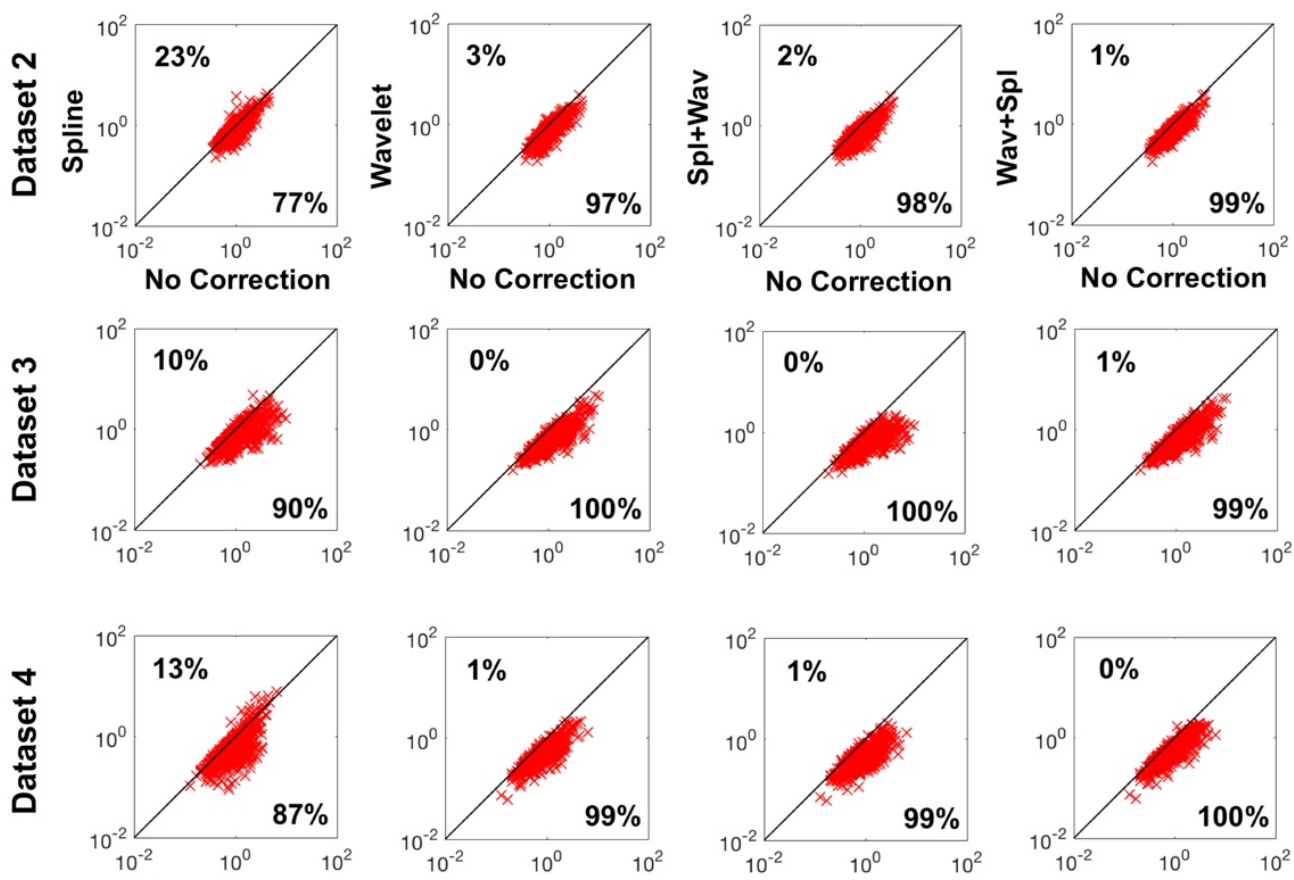

Figure 2.12 Scatter plots of the between-subjects (upper panel) and within-subject (lower panel) SD computed for all the motion correction techniques ( $y$ axis) versus no motion correction ( $\mathrm{x}$ axis) for $\mathrm{HbO}$ of datasets 2, 3 and 4 (rows 1, 2, and 3 of each panel). Spl+Wav and Wav+Spl have the best performance in reducing the within and between standard deviation across all datasets (i.e., SD reduced by $100 \%$.ca of the cases). 


\section{Discussion}

The primary objective of this work is to provide recommendations for researchers who first approach infant fNIRS data analysis. fNIRS infant recordings are often contaminated by motion artifacts of various shapes and frequencies that are not easy to detect and consequently correct. To our knowledge, only one recent study measured the effects of a selection of motion correction techniques on infant data (Behrendt et al., 2018). Our study contributes to the current literature in two ways. First, by comparing the performance of most motion correction techniques available in Homer2 on infants' semi-simulated data and by further assessing their performance based on the percentage of motion artifacts present in the signal. Second, by testing the efficacy of a novel combination of motion correction methods, Spline interpolation and Wavelet filtering, on real infant data collected from different sites using different paradigms, headgears and NIRS systems.

\section{Motion correction performance in semi-simulation}

To begin with, we tested the effect of changing the iqr values (i.e., $0.1,0.5,0.8,1.0,1.2$, 1.5) of the Wavelet motion correction technique on three different types of simulated HRFs (previously defined as three sets of data: Short, Block Design, Standard HRFs) that were added to infant resting state recordings. Two iqr values ( 0.5 and 0.8$)$ tended to best recover the majority of trials and improved the overall estimation of the HRF across all sets of data. Subsequently, we evaluated the performance of all the tested motion correction techniques. Trial Rejection and No Correction were the techniques with the worst performance in recovering trials and HRFs in all three sets of data. This is in line with the findings from previous studies on adult and infant data (Behrendt et al., 2018; Brigadoi et al., 2014), that also discourage the use of rejection of trials affected by motion artifacts as main correction strategy. This approach might be effective only in cases where motion artifacts are not frequent (Brigadoi et al., 2014) and a high number of trials are available, which is not common in infant studies. 
Of the motion correction algorithms, Spline SG showed the worst performance as it did not recover any of the trials. One possible reason for this is that the spline part of this method is only applied when SNR $>3$. Thus, given the low SNR typical of infant data, the SG part was more often applied to our datasets alone than in combination with spline (an average of $30 \%$ of channels per subject showed SNR $>3$ in this dataset, with 7 infants not showing any channel with SNR $>3$ ). The use of this smoothing filter on its own might not have been sufficient to correct the artifacts or might even alter the signal resulting in larger artifacts. The second worst performing technique was Wavelet Kurtosis. For both metrics (number of recovered trials and RMSE) its performance was similar to that of trial rejection. In light of these findings, we did not test either Spline SG or Wavelet Kurtosis on the task-based datasets and we do not recommend the use of either correction algorithms for the analysis of infant data.

The techniques with the best performance on both metrics were tPCA and the combinations of Spline and Wavelet. A key finding of this first set of analyses is that the combinations of Spline and Wavelet, compared to the same techniques on their own, were able to recover most of the trials regardless of iqr (0.5 or 0.8$)$ and order (Spl+Wav or Wav+Spl), with Spl+Wav being the method with the lowest RMSE median and, on average, the highest number of recovered trials. These results suggest that the combination represents a valid option for recovering the HRF across different types of datasets. In their study, Behrendt and colleagues (2018) tested Wavelet filtering with iqrs of $0.1,0.5,1.0$ and 1.5 on infant data and recommended the use of 0.5 . While our results do not entirely diverge from this suggestion, we advise infant researchers to try iqr $=0.5$ and $i q r=0.8$, and choose the value that prevents the risk of underestimating the true haemodynamic response. We suggest to use $i q r=0.5$ when analyzing data collected with stimuli of $10 \mathrm{~s}$ or longer, and $i q r=0.8$ when analyzing short event-related data (i.e., collected with stimuli shorter than $10 \mathrm{~s}$ ).

One technique that, despite being available in Homer2, was not included in our analysis was CBSI. The reason for this choice reflects the concerns previously raised by Brigadoi and colleagues (2014), namely that CBSI does not correct HbR starting from 
the original signal, but creates its surrogate from $\mathrm{HbO}$ (therefore $\mathrm{HbR}$ does not reflect real measured data). Further, CBSI relies on strict assumptions on the relation between $\mathrm{HbO}$ and $\mathrm{HbR}$ that are not always met, and when this happens, the performance of this technique is negatively affected (Brigadoi et al., 2014).

The superiority of the combination of Spline and Wavelet has been further confirmed by the analysis computed on data containing from $0 \%$ to $21 \%$ of artifacts. Namely, we found that when no artifacts were detected, all the techniques had a similar performance in recovering the HRF, whereas at increasing percentages of artifacts Wavelet (at $i q r=0.5)$, tPCA and particularly Spl+Wav were more effective compared to the other techniques. Our findings are in contrast with the work by Berendt and colleagues (2018), confirming that tPCA performs better than Wavelet also with a higher presence of artifacts in the data. The reason of these conflicting results might be related to the choice of input parameters required for tPCA; perhaps the parameters used in the current work were more efficient in identifying the majority of artifacts in Dataset 1 as compared to the parameters used by Behrendt and colleagues (2018) in their dataset. A negative feature of tPCA is its dependence on the number of available channels; when more channels are available this usually leads to a better correction of the signal. However, infant research sometimes uses only few channels (e.g., Kida \& Shinohara, 2013; Minagawa et al., 2008). Therefore, researchers should be aware of this tPCA feature when deciding which motion correction technique to apply, particularly when their dataset has few channels available. In this work, we aimed to evaluate and suggest a new stacking approach that can be used with noisy infant data acquired with all types of probe arrays (i.e., multi- or single-channel arrays); hence, we discarded tPCA in the tests performed on the task-based datasets.

In sum, our findings on Dataset 1 suggest that Spl+Wav might be the optimal correction method for the recovery of the true HRF in infant semi-simulated data, at all percentages of motion artifacts. 


\section{Motion correction performance in the task-based datasets}

The results of the comparison between the different correction methods on infant data collected during cognitive/perceptual tasks (i.e., Datasets 2, 3, 4), support the findings of our first analyses on semi-simulated infant data. Also in this case, rejection and No Correction are the worst methods in reducing artifacts. Spline, when compared to its combination with Wavelet or to Wavelet on its own, had the worst performance in reducing the within- and between-subjects SDs and in recovering a sufficient number of trials in all three datasets. On the other hand, the use of Wavelet alone seems to work well in reducing the within- and between-subjects SDs across all datasets; however, this technique also saves less trials from rejection in comparison to the combined use of Wavelet and Spline. In infant research, including the maximum number of valid trials in the data analysis is crucial for obtaining a reliable haemodynamic response, and, ultimately, for reaching sufficient power in the statistical analyses.

\section{General Conclusions}

In the current study we used four different metrics (i.e., RMSE; within- and betweensubjects SD; number of recovered trials) to evaluate and compare the performance of different motion correction techniques in infant data. As each metric holds strengths and limitations (e.g., within-subject SD could be affected by habituation) conclusions on the effectiveness of the correction methods can only be drawn by taking into account the results obtained for all metrics. Therefore, in this section we will further discuss evidence coming from tests on all datasets and all metrics.

The combination of Spline and Wavelet was the most effective in (1) recovering the true HRF in infant semi-simulated data, both at low and high percentages of motion artifacts; (2) reducing the within- and between-subjects standard deviations; (3) saving nearly all trials across the three task-based datasets. Therefore, for infant data containing a large number of motion artifacts we suggest the combined use of Spline and Wavelet. Moreover, although we found that the optimal order of these correction methods differs per dataset, we suggest the application of Spline followed by Wavelet since this order showed a slightly better recovery of the true HRF in semi-simulated data (i.e., lowest 
RMSE median).

Given that the combined use of Spline and Wavelet had an optimal performance on all datasets that varied from one another on a number of levels (percentage of artifacts, age, task, NIRS system, headgear), we can conclude that our findings can be safely generalized to any infant dataset and, possibly, also to datasets acquired from other challenging samples, such as clinical population, under the hypothesis that several unpredictable motion artifacts could be present in these clinical acquisitions, coupled with few available trials. Our findings in semi-simulation show that the stacking recovered the true HRF better than when applying Wavelet alone. This result could apparently be in contrast with the results of Behrendt and colleagues (2018) who reported that the recovery of the haemodynamic response was not improved by the combination of tPCA and Wavelet compared to when only Wavelet was applied. The difference between the two studies could be identified in the differences between the modus operandi of tPCA and Spline. Similarly to Spline, tPCA works on predefined motion artifacts and performs well in correcting step changes in the signal (Yücel, Selb, Cooper, \& Boas, 2014); conversely, tPCA applies the correction to all channels, including those that are not affected by motion, which might result in an undesired modification of the original signal or even a loss of useful signal in multiple channels when many motion artifacts sparsely located across channels are present.

Whereas we suggest the use of Spline and Wavelet in combination, we advise caution when selecting the parameters of the motion detection function (hmrMotionArtifactbyChannel function in Homer2). This step is crucial since the performance of Spline is highly dependent on the detection of the motion artifacts present in the data, which is determined, in turn, by the chosen values. Specifically, we found that the STDEV thresh was the parameter that needed most careful tuning. In this work, the STDEVthresh value was defined by visually evaluating how efficient the function was in identifying the major motion artifacts present in the data. While this procedure is necessary to correctly select the motion artifacts, it is time consuming and subjective. Future work should address these issues by creating a more automatic and 
objective way to define these parameters.

On this note, we take the opportunity to also stress the importance of carefully selecting the parameters of another user-dependent function: the band-pass filter (for more in depth discussion on filter selection see Pinti, Scholkmann, Hamilton, Burgess, \& Tachtsidis, 2018). The choice of appropriate cut-off frequencies is critical to preserve haemodynamic responses evoked by experimental conditions while excluding irrelevant frequencies associated with physiological oscillations. In infant research, it is also important to take into account the age of the participants when setting the filter cut-offs, as the frequency of some of these oscillations (e.g., heart rate) change during development. While we are confident that the slightly different filter cut-offs employed in all datasets tested in the current study did not impact our motion correction results, which are consistent across datasets, we suggest that future studies should investigate which is the most appropriate combination of motion correction technique and bandpass filter for infant fNIRS data.

Despite our findings are in favor of the combined use of Spline and Wavelet, we do not discourage the application of Wavelet as exclusive correction method. Our results confirmed previous findings that Wavelet on its own has a better performance compared to Spline interpolation (Brigadoi et al., 2014). We showed that this technique is a good option with moderately noisy datasets but the combination of Spline and Wavelet should be preferred as the percentage of noise in the signal increases. Therefore, we suggest that the decision of which technique to apply should be driven by a critical evaluation of the number and characteristics of artifacts embedded in the fNIRS time courses. One benefit of using Wavelet filtering alone is that it does not require the detection of motion artifacts, a challenging task when the artifacts affecting the NIRS recordings are extremely difficult to detect using automatic methods. We do not advise including Spline as sole correction method in the preprocessing of infant data. Specifically, we showed that Spline, compared to Wavelet and to the stacking of Spline and Wavelet, had the worst performance in recovering the true HRF in semi-simulated data (highest RMSE), in lowering the within- and between-subjects SDs in the task- 
based datasets and in recovering an acceptable number of trials in all four datasets.

To summarize, the convergence of results across all the different datasets tested in our work does not only indicate that the efficacy of the combination of Spline and Wavelet is independent from infants' age, the task design (visual/auditory vs. tactile stimulation), or percentage of noise, but that it is not determined either by the fNIRS system and headgear used.

It is likely that in the near future, thanks to technological advances such as the development of lightweight fibers or portable devices, we will witness an increase in the number of channels used for infants studies and, eventually, full head coverage arrays. While in this work we did not test the performance of our techniques on data collected with large channel arrays we believe that the same recommendations can be applied to such datasets. As long as the headgear is tightly fitted to the subject's head, the data quality should be comparable to the datasets tested in the present work.

\section{Acknowledgements}

The authors would like to thank the infants and their families for their participation. The authors would like to thank Prof. Livio Finos for the fruitful discussion and advices on statistical analyses.

Dataset 1 was supported by the Leverhulme Trust Research Project Grant (2015-115). Data was collected by Chiara Bulgarelli and Dr. Carina De Klerk. The authors would like to thank Professor Victoria Southgate and Dr. Antonia Hamilton as PIs of the project for sharing the data.

Dataset 2 was supported by the UK Medical Research Council (G0701484), the Simons Foundation (no. SFARI201287), the BASIS Funding Consortium Led by Autistica (www.basisnetwork.org) and the Innovative Medicines Initiative Joint Undertaking under grant agreement no. 115300, resources of which are composed of financial contribution from the European Union's Seventh Framework Programme (FP7/20072013) and EFPIA companies. The BASIS team in alphabetical order: Baron-Cohen, S., Bedford, R. Bolton, P., Blasi, A., Charman, T., Cheung, H.M., Davies, K., Elsabbagh, M., 
Fernandes, J., Gammer, I., Gliga, T., Green, J., Guiraud, J., Johnson, M.H., Liew, M., Lloyd-Fox, S., Maris, H., O’Hara, L., Pasco, G., Pickles, A., Ribeiro, H., Salomone, E., Tucker, L., Yemane, F.

Dataset 3 was supported by a grant from the European Community's Horizon 2020 Program under grant agreement $n^{\circ} 642996$ (Brainview) (RDL). The authors would like to thank Rianne van Rooijen for helping with the NIRS data acquisition, Carlijn van den Boomen for lab support and Professor Chantal Kemner as PI of the project for providing lab space and equipment.

Dataset 4 was supported by a grant from a Grant-in-Aid for Scientific Research (A) (24118508) (YM) and MEXT Supported Program for the Strategic Research Foundation at Private Universities. The authors would like to thank Aika Yasui for the fNIRS data collection.

S.B. was supported by grant "Progetti di Ateneo Bando 2015" C92I1600012005 and by "Progetto STARS Grants 2017" C96C18001930005 both from the University of Padova. 


\section{References}

Abdelnour, A. F., \& Huppert, T. J. (2009). Real-time imaging of human brain function by near-infrared spectroscopy using an adaptive general linear model. NeuroImage, 46(1), 133-143. http://doi.org/10.1016/j.neuroimage.2009.01.033

Aslin, R., Shukla, M., \& Emberson, L. L. (2015). Hemodynamic Correlates of Cognition in Human Infants. Annu Rev Psychol., 33(4), 395-401. http://doi.org/10.1038/nbt.3121

Behrendt, H. F., Firk, C., Nelson, C. A., \& Perdue, K. L. (2018). Motion correction for infant functional near-infrared spectroscopy with an application to live interaction data. Neurophotonics, 5(01), 1. http://doi.org/10.1117/1.NPh.5.1.015004

Brigadoi, S., Ceccherini, L., Cutini, S., Scarpa, F., Scatturin, P., Selb, J., ... Cooper, R. J. (2014). Motion artifacts in functional near-infrared spectroscopy: A comparison of motion correction techniques applied to real cognitive data. NeuroImage, 85, 181-191. http://doi.org/10.1016/j.neuroimage.2013.04.082

Bulgarelli, C., Blasi, A., Arridge, S., Powell, S., de Klerk, C. C., Southgate, V., ... \& Hamilton, A. (2018). Dynamic causal modelling on infant fNIRS data: A validation study on a simultaneously recorded fNIRS-fMRI dataset. NeuroImage, 175, 413-424. https://doi.org/10.1016/j.neuroimage.2018.04.022

Chiarelli, A. M., Maclin, E. L., Fabiani, M., \& Gratton, G. (2015). A kurtosis-based wavelet algorithm for motion artifact correction of fNIRS data. NeuroImage, 112, 128-137. http://doi.org/10.1016/j.neuroimage.2015.02.057

Cooper, R. J., Selb, J., Gagnon, L., Phillip, D., Schytz, H. W., Iversen, H. K., ... Boas, D. A. (2012). A systematic comparison of motion artifact correction techniques for functional near-infrared spectroscopy. Frontiers in Neuroscience, 6(OCT), 1-10. http://doi.org/10.3389/fnins.2012.00147

Cope, M., \& Delpy, D. T. (1988). System for long-term measurement of cerebral blood and tissue oxygenation on newborn infants by near infra-red transillumination. Medical and Biological Engineering and Computing, 26(3), 289-294.

Delpy, D. T., Cope, M., van der Zee, P., Arridge, S., Wray, S., \& Wyatt, J. (1988). Estimation of optical pathlength through tissue from direct time of flight measurement. Physics in Medicine and Biology, 33(12), 1433-1442. http://doi.org/10.1088/0031-9155/33/12/008

Di Lorenzo, R., Blasi, A., Junge, C., van den Boomen, C., van Rooijen, R., \& Kemner, C. (2019). Brain responses to faces and facial expressions in 5-month-olds: an fNIRS study. Frontiers in Psychology, 10, 1240. https://doi.org/10.3389/fpsyg.2019.01240

Duncan, a, Meek, J. H., Clemence, M., Elwell, C. E., Tyszczuk, L., Cope, M., \& Delpy, D. T. (1995). Optical pathlength measurements on adult head, calf and forearm and the head of the newborn infant using phase resolved optical spectroscopy. Physics in Medicine and Biology, 40(2), 295-304. http://doi.org/10.1088/0031-9155/40/2/007 
Everdell, N. L., Gibson, A. P., Tullis, I. D. C., Vaithianathan, T., Hebden, J. C., \& Delpy, D. T. (2005). A frequency multiplexed near-infrared topography system for imaging functional activation in the brain. Review of Scientific Instruments, 76(9), 093705. http://doi.org/10.1063/1.2038567

Issard, C., \& Gervain, J. (2018). Variability of the hemodynamic response in infants: Influence of experimental design and stimulus complexity. Developmental cognitive neuroscience, 33, 182-193. https://doi.org/10.1016/j.dcn.2018.01.009

Grossmann, T., Johnson, M. H., Lloyd-Fox, S., Blasi, A., Deligianni, F., Elwell, C., \& Csibra, G. (2008). Early cortical specialization for face-to-face communication in human infants. Proceedings of the Royal Society B: Biological Sciences, 275(1653), 2803-2811.

Homae, F., Watanabe, H., Otobe, T., Nakano, T., Go, T., Konishi, Y., \& Taga, G. (2010). Development of global cortical networks in early infancy. Journal of Neuroscience, 30(14), 4877-4882. https://doi.org/10.1523/JNEUROSCI.5618-09.2010

Hu, X.-S., Arredondo, M. M., Gomba, M., Confer, N., DaSilva, A. F., Johnson, T. D., ... Kovelman, I. (2015). Comparison of motion correction techniques applied to functional near-infrared spectroscopy data from children. Journal of Biomedical Optics, 20(12), 126003. http://doi.org/10.1117/1.JBO.20.12.126003

Huppert, T. J., Diamond, S. G., Franceschini, M. A., \& Boas, D. A. (2009). Hom\{ER\}: a review of time-series analysis methods for near-infrared spectroscopy of the brain. Appl Opt, 48(10), D280--D298. http://doi.org/10.1364/AO.48.00D280

Jahani, S., Setarehdan, S.K., Boas, D.A. and Yücel, M.A., 2018. Motion artifact detection and correction in functional near-infrared spectroscopy: a new hybrid method based on spline interpolation method and Savitzky-Golay filtering. Neurophotonics, 5(1), 015003. https://doi.org/10.1117/1.NPh.5.1.015003

Kida, T., \& Shinohara, K. (2013). Gentle touch activates the prefrontal cortex in infancy: An NIRS study. Neuroscience Letters, 541, 63-66.

http://doi.org/10.1016/j.neulet.2013.01.048

Lloyd-Fox, S., Blasi, A., Volein, A., Everdell, N., Elwell, C. E., \& Johnson, M. H. (2009). Social perception in infancy: a near infrared spectroscopy study. Child development, 80(4), 986-999. http://doi.org/10.1111/j.1467-8624.2009.01312.x

Lloyd-Fox, S., Blasi, A., \& Elwell, C. E. (2010). Illuminating the developing brain: The past, present and future of functional near infrared spectroscopy. Neuroscience and Biobehavioral Reviews, 34(3), 269-284. http://doi.org/10.1016/j.neubiorev.2009.07.008

Lloyd-Fox, S., Blasi, A., Pasco, G., Gliga, T., Jones, E. J. H., Murphy, D. G. M., ... Yemane, F. (2018). Cortical responses before 6 months of life associate with later autism. European Journal of Neuroscience, 47(6), 736-749. http://doi.org/10.1111/ejn.13757 
Lloyd-Fox, S., Széplaki-Köllod, B., Yin, J., \& Csibra, G. (2015). Are you talking to me? Neural activations in 6-month-old infants in response to being addressed during natural interactions. Cortex, 70, 35-48. http://doi.org/10.1016/j.cortex.2015.02.005

Miguel, H. O., Lisboa, I. C., Gonçalves, O. F., \& Sampaio, A. (2017). Brain mechanisms for processing discriminative and affective touch in 7-month-old infants. Developmental Cognitive Neuroscience, (February), 0-1. http://doi.org/10.1016/j.dcn.2017.10.008

Minagawa-Kawai, Y., Matsuoka, S., Dan, I., Naoi, N., Nakamura, K. and Kojima, S., 2008. Prefrontal activation associated with social attachment: facial-emotion recognition in mothers and infants. Cerebral Cortex, 19(2), pp.284-292.

https://doi.org/10.1093/cercor/bhn081

Molavi, B., \& Dumont, G. A. (2012). Wavelet-based motion artifact removal for functional near-infrared spectroscopy. Physiological Measurement, 33(2), 259-270. http://doi.org/10.1088/0967-3334/33/2/259

Molavi, B., May, L., Gervain, J., Carreiras, M., Werker, J. F., \& Dumont, G. A. (2014). Analyzing the resting state functional connectivity in the human language system using near infrared spectroscopy. Frontiers in Human Neuroscience, 7(January), 1-9. http://doi.org/10.3389/fnhum.2013.00921

Obrig, H., \& Villringer, A. (2003). Beyond the visible - Imaging the human brain with light. Journal of Cerebral Blood Flow and Metabolism, 23(1), 1-18. http://doi.org/10.1097/01.WCB.0000043472.45775.29

Pinti, P., Scholkmann, F., Hamilton, A., Burgess, P., \& Tachtsidis, I. (2018). Current status and issues regarding pre-processing of fNIRS neuroimaging data: An investigation of diverse signal filtering methods within a General Linear Model framework. Frontiers in human neuroscience, 12. http://doi.org/10.3389/fnhum.2018.00505

Ravicz, M. M., Perdue, K. L., Westerlund, A., Vanderwert, R. E., \& Nelson, C. A. (2015). Infants' neural responses to facial emotion in the prefrontal cortex are correlated with temperament: a functional near-infrared spectroscopy study. Frontiers in Psychology, 6(July), 1-12. http://doi.org/10.3389/fpsyg.2015.00922

RStudio Team (2016). RStudio: Integrated Development for R. RStudio, Inc., Boston, MA URL http://www.rstudio.com/

Scholkmann, F., Spichtig, S., Muehlemann, T., \& Wolf, M. (2010). How to detect and reduce movement artifacts in near-infrared imaging using moving standard deviation and spline interpolation. Physiological measurement, 31(5), 649-662.

http://doi.org/10.1088/0967-3334/31/5/004

Timeo, S., Brigadoi, S., \& Farroni, T. (2017). Perception of Caucasian and African faces in 5-to 9-month-old Caucasian infants: A functional near-infrared spectroscopy study. Neuropsychologia. http://doi.org/10.1016/j.neuropsychologia.2017.09.011 
Wilcox, T., \& Biondi, M. (2015). fNIRS in the developmental sciences. Wiley Interdisciplinary Reviews: Cognitive Science, 6(3), 263-283. http://doi.org/10.1002/wcs.1343

Yücel, M. A., Selb, J., Cooper, R. J., \& Boas, D. A. (2014). Targeted principle component analysis: a new motion artifact correction approach for near-infrared spectroscopy. Journal of innovative optical health sciences, 7(02), 1350066. https://doi.org/10.1142/S1793545813500661

Zhang, Y., Brooks, D. H., Franceschini, M. A., \& Boas, D. A. (2005). Eigenvector-based spatial filtering for reduction of physiological interference in diffuse optical imaging. Journal of biomedical optics, 10(1), 011014. https://doi.org/10.1117/1.1852552 


\section{Supplementary Materials I}

We also performed analyses on $\mathrm{HbR}$ values computed from the four datasets included in this work. Overall, these results are in line with the results obtained for $\mathrm{HbO}$ values.

\section{Motion correction performance in simulated HRFs (Dataset 1)}

\section{Tuning parameters of Wavelet}

Figure S2.1 reports the performance of the Wavelet motion correction technique across iqr values for the RMSE metric. The RMSE was significantly different among iqr values in the "Standard HRF" set of data $(F(5,55)=4.90, p<.001)$; paired $t$-tests revealed that the RMSE was significantly larger for iqr 1.5 when compared to iqr $1.2(p<.05)$. No significant differences among techniques were found in the "Short HRF" and in the “Block Design HRF" sets of data $(\max F=1.61, \min p=.17)$.
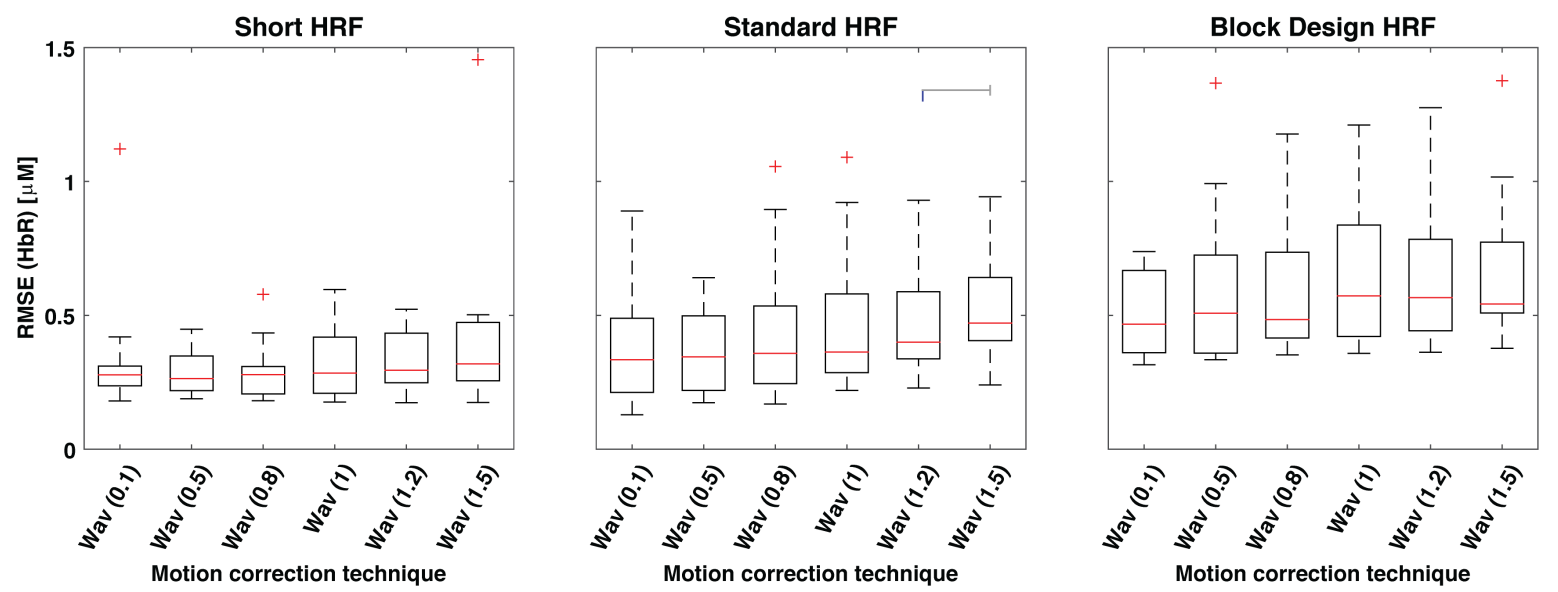

Figure S2.1 RMSE recovered after applying the Wavelet motion correction technique with different iqr values for each set of data of Dataset 1 . The red line in the box plots depicts the median value, the two whiskers denote the first and third quartile and outliers are represented by red crosses. The line above the "Standard HRF" box plot, indicates the only significant statistical difference found between techniques, that is, iqr 1.5 vs. $1.2(p<.05)$. 


\section{Performance of correction techniques in simulated HRFs}

Figure S2.2 reports the RMSE obtained after applying each motion correction processing pipeline. RMSE was significantly different among techniques in all three sets of data $(\min F=2.31, \max p=.017)$; the $\mathrm{t}$-tests between techniques are reported in Figure S2.2. Note that the results relative to the performance of Spline Savitzky-Golay are not shown in this and the following figures since the application of this technique had a strong impact on the signal, causing the rejection of all the trials in all subjects. As for $\mathrm{HbO}$ values, also the analyses performed on $\mathrm{HbR}$ showed that trial rejection, No Correction, and Wavelet Kurtosis were the methods with the worst performance across all sets of data. For HbR values, also Spline had a poor performance in lowering the RMSE. tPCA, Wavelet and the combination of Spline and Wavelet had a similar performance. However, the method scoring the lowest median RMSE and showing the smallest range, for all sets of data, was the combination of Spline (performed at first step) and Wavelet (performed at second step).
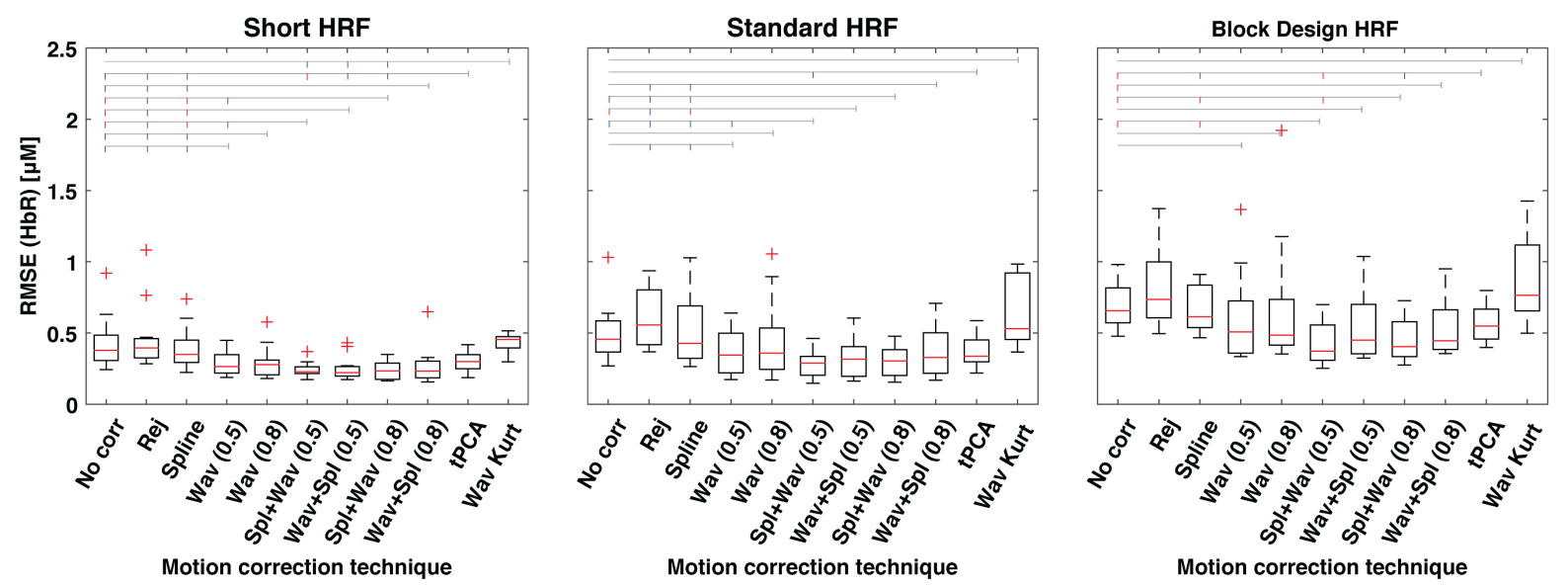

Figure S2.2 RMSE for each processing pipeline and for each of the three sets of data of Dataset1. The red line in the box plots depicts the median value, the two whiskers denote the first and third quartile and outliers are represented by red crosses. The lines above linking the different techniques indicate the significant statistical difference: blue lines correspond to $p<.05$ and red lines to $p<.01$.

Figure S2.3 reports example individual average HRFs for one channel (the same of Figure 2.6) recovered after applying the different motion correction techniques on the three sets of data. 

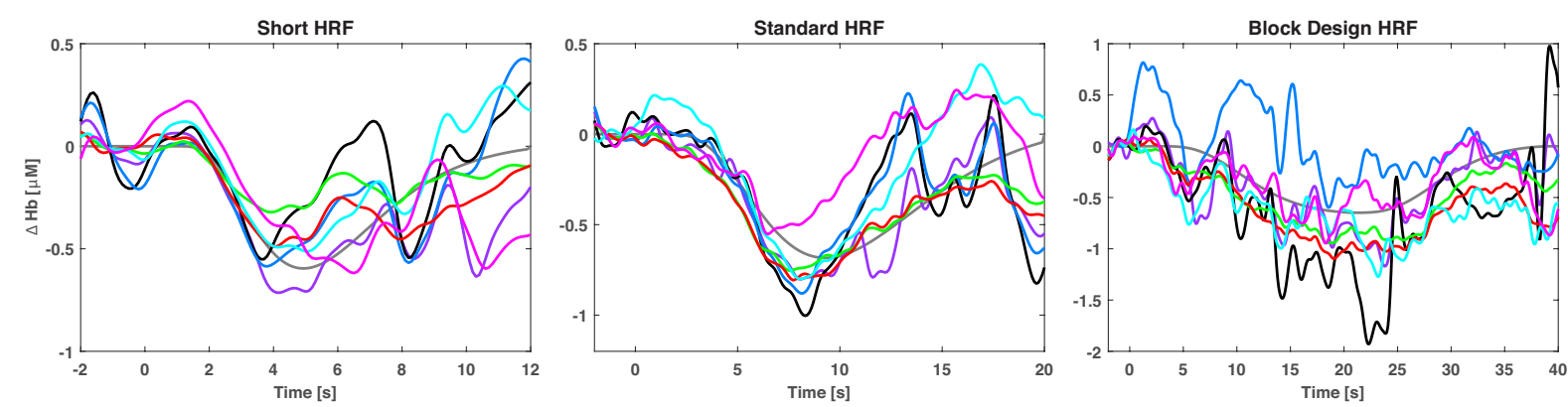

Figure S2.3 Examples of individual average HbR responses for one channel recovered after applying different motion correction approaches on the three sets of data of Dataset 1.

\section{Analyses on tPCA (HbO and HbR)}

In this set of analysis we tested whether the number of channels influenced the performance of tPCA in recovering the true HRF in infant data. Both the number of recovered trials and the RMSE values were employed as metric for comparison. In particular, we compared the RMSE values obtained when tPCA was applied on approximately half the number of channels available (tPCA Half Figure S2.4), with the RMSE values calculated for the same channel selection of tPCA Half, but after applying tPCA on the entire dataset (tPCA in Figure S2.4). The box plots in Figure S2.4 show that, although non statistically significantly different, tPCA and tPCA Half yield different results, suggesting that tPCA efficacy is dependent on the number of channels available. 
a)

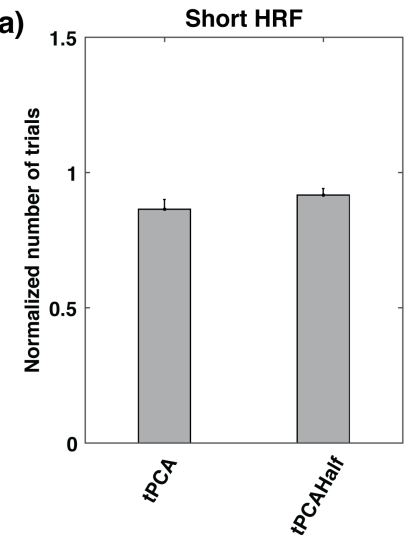

Motion correction technique

b)
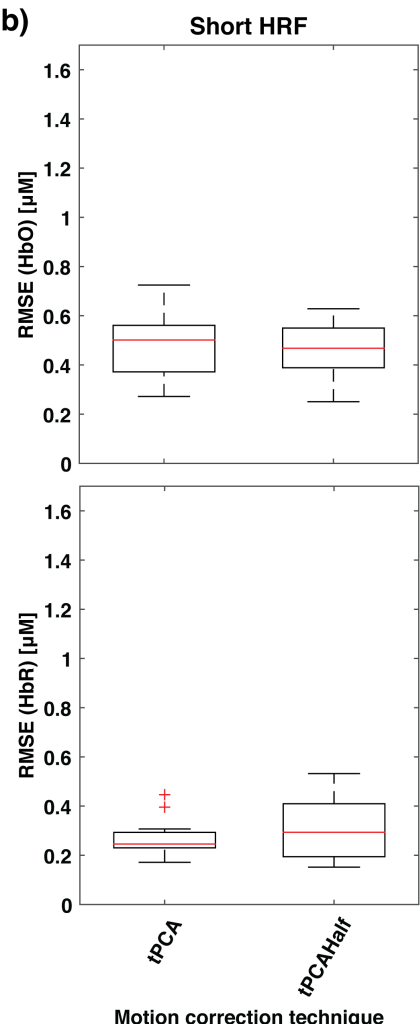

Standard HRF

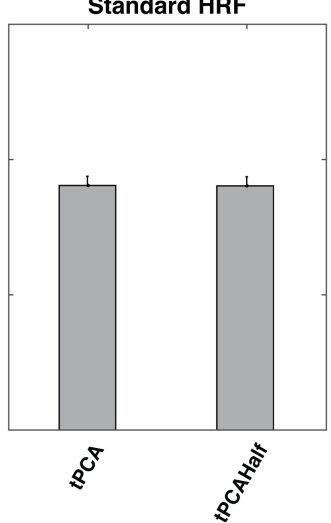

Motion correction technique Standard HRF
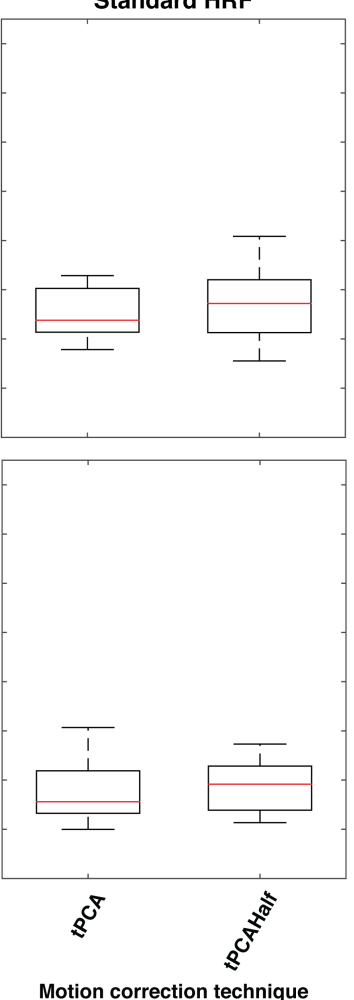

Block Design HRF

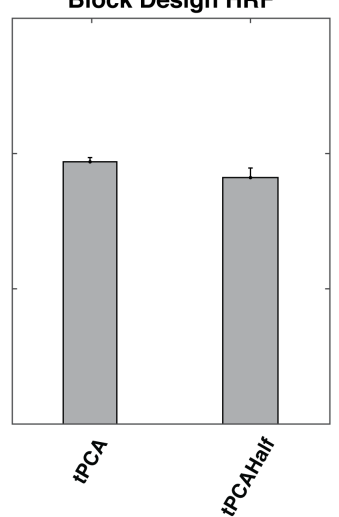

Motion correction technique

Block Design HRF
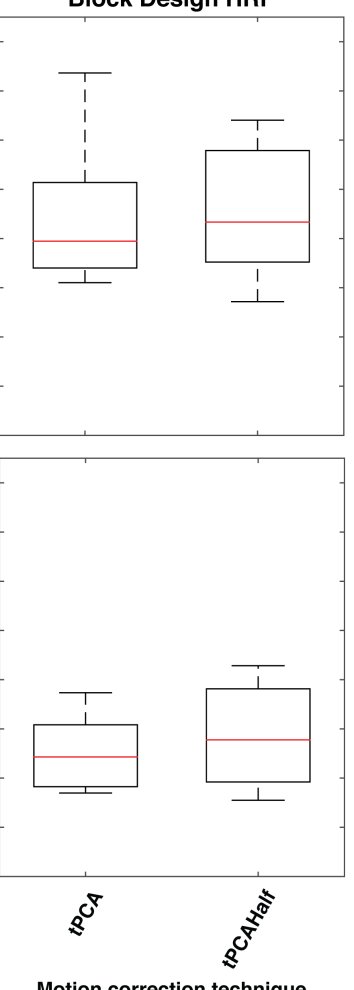

Figure S2.4 Normalized number of trials (a) and RMSE (b) recovered after tPCA was applied on the full range of channels $(\mathrm{N}=30)$ or only on approximately half the number of available channels $(n=11$; tPCA Half), for each of the three sets of data of Dataset 1, for HbO (upper row panel b) and HbR (lower row panel b) values. In panel a, error-bars represent standard error across participants. In panel b, the red line in the box plots depicts the median value, the two whiskers denote the first and third quartile and outliers are represented by red crosses. 
Chapter 2

\section{Motion correction performance at different percentages of motion artifact}

\section{contamination}

Results of these analyses were similar to those on $\mathrm{HbO}$ values (see figures S2.5, S2.6).

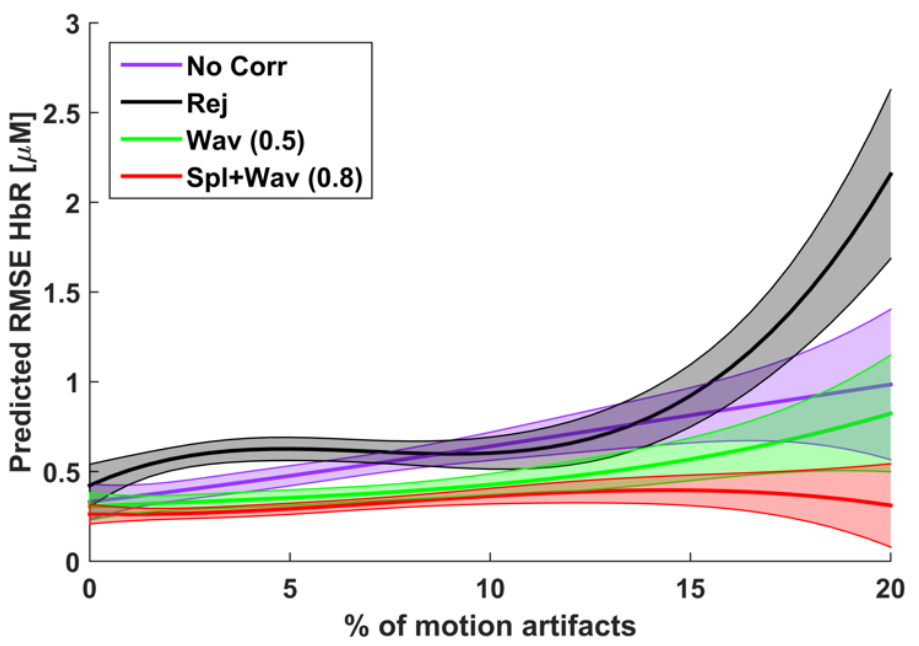

Figure S2.5 Estimated RMSE HbR values at different percentages of motion artifact contamination for a selection of motion correction methods. Rejection, No Correction, Wav (0.5) and Spl+Wav (0.8) were selected for visualization purposes. The shaded areas represent the confidence intervals.
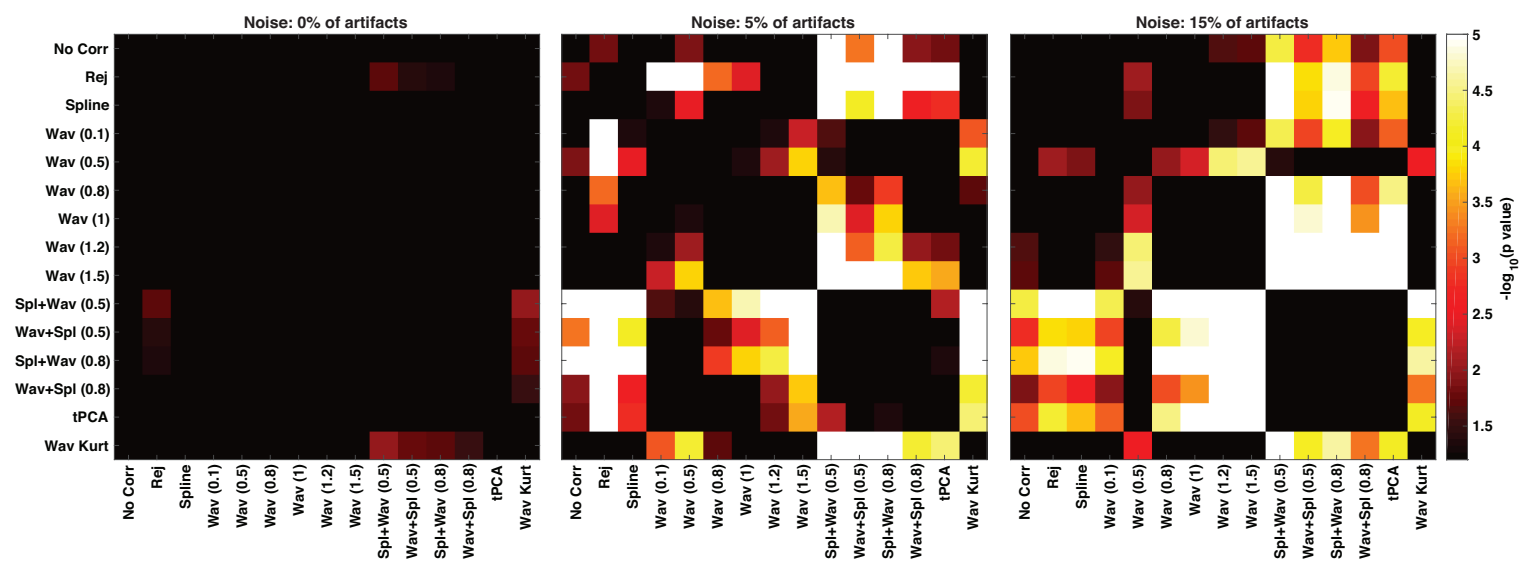

Figure S2.6 Corrected p values resulting from the paired t-tests computed between any pair of correction methods on the RMSE HbR values of the "Standard HRF" dataset at $0 \%, 5 \%$, and $15 \%$ of artifact contamination. For visualization purposes, we selected only 3 levels of noise for one HRF type. The colorbar represents $-\log 10$ ( $\mathrm{p}$ value) for visualization purposes (the value 1.2 , which is the lower limit, corresponds to $p=.05$, higher values correspond to $p s<.05$, the higher the more significant). 


\section{Motion correction performance in task-based datasets (Datasets 2, 3, 4)}

For every dataset, the repeated measures ANOVA performed on the within-subject SDs revealed a main effect of technique (all $p s<.0001$ ). In general, across all datasets, No Correction, Rejection and Spline performed poorly in reducing the within-subjects SDs, while Wavelet and the two possible combinations of Spline and Wavelet reduced more effectively this measure (Figure S2.7).
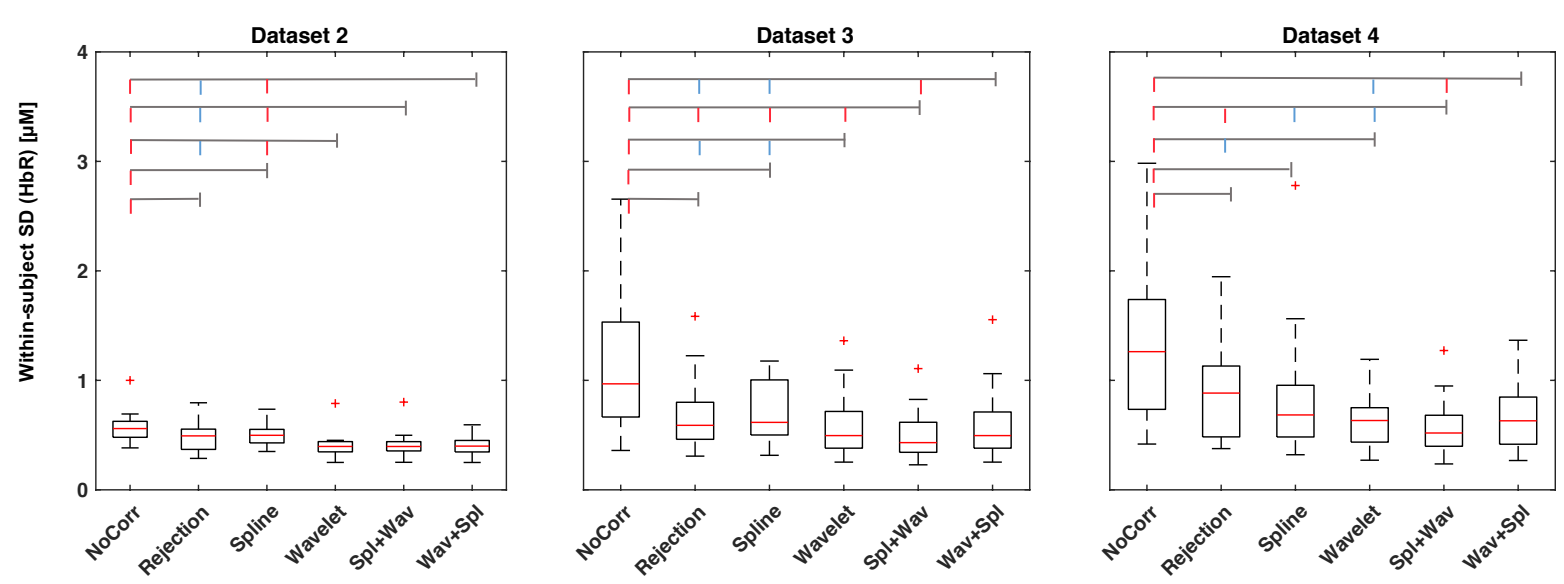

Figure S2.7 Box plots of the within-subject SD for HbR values of all techniques of Datasets 2, 3, 4. The red line in the box plots indicates the median, the two whiskers denote the first and third quartile, the red crosses indicate outliers. The lines above linking the different techniques denote significant statistical difference: blue lines correspond to $p<.05$ and red lines to $p<.01$.

\section{Performance of Rejection versus No Correction}

The between-subjects SD scatter plots (first column of Figure S2.8) show that trial rejection performs worse than No Correction across all datasets, increasing the SD among the subjects' mean HRF more than $66 \%$ of the time. This result, as discussed for $\mathrm{HbO}$ values, could be due to the presence of some very noisy mean HRFs measured from data with many rejected trials. On the contrary, Rejection performs better than No Correction in reducing the within-subject SD (second column of Figure S2.8); the rejection of trials affected by motion artifacts is indeed efficient in reducing the variability between trials within each subject. For all datasets, paired t-tests supported this evidence, showing a significant difference between these two techniques (all $p s<.01$; see Figure S2.7). 

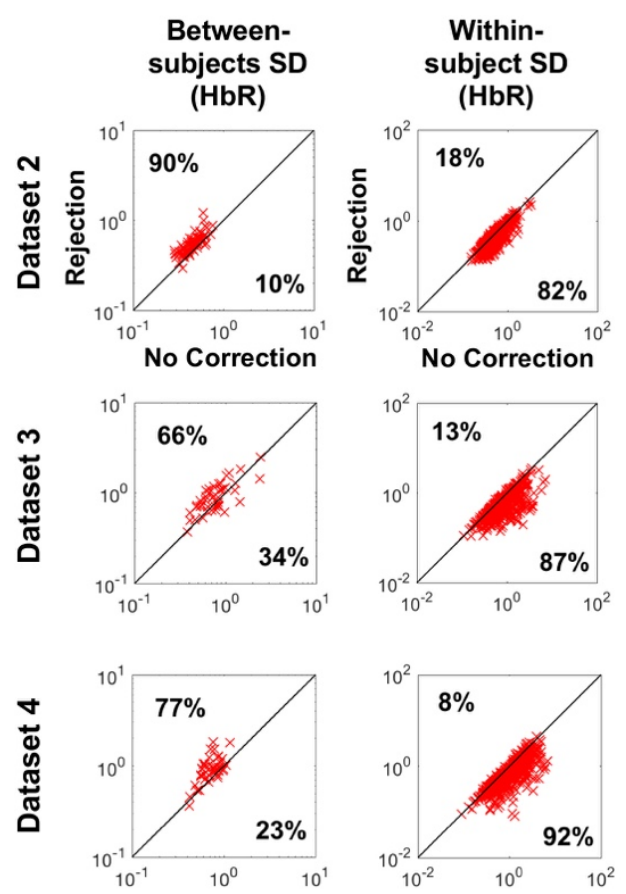

Figure S2.8 Scatter plots of the between-subjects (left column) and within-subject (right column) SD calculated for the comparison between rejection (y axis) and No Correction technique ( $\mathrm{x}$ axis) for HbR values of datasets 2,3 and 4 (row 1,2,3, respectively).

\section{Performance of Motion Correction techniques versus No Correction}

All the correction methods significantly reduced the within-subject SD across all datasets (Figure S2.7) (paired t-tests No Correction vs. correction, all $p s<.01$ ). In particular, we found that Wavelet and both combinations (Wav+Spl and Spl+Wav) performed best at reducing the standard deviation in $93 \%$ to $100 \%$ of cases (Figure S2.9 lower panel). Out of the techniques we tested, Spline reduced the SD in $80 \%$ to $92 \%$ of the cases (Figure S2.9 lower panel). Two-tailed paired t-tests performed between all the correction methods are reported in Figure S2.7, which overall confirm that Wavelet and its use in combination with Spline performs best in motion reduction as compared to other techniques.

The between-subjects SD was reduced in $100 \%$ of cases with Wav+Spl in all datasets, with Spl+Wav performing similarly (Figure S2.9 upper panel). As found for $\mathrm{HbO}$ values, techniques on their own have a lower power in reducing the betweensubjects SD, with Spline having the worst performance. 


\section{Between-subjects SD Correction HbR}
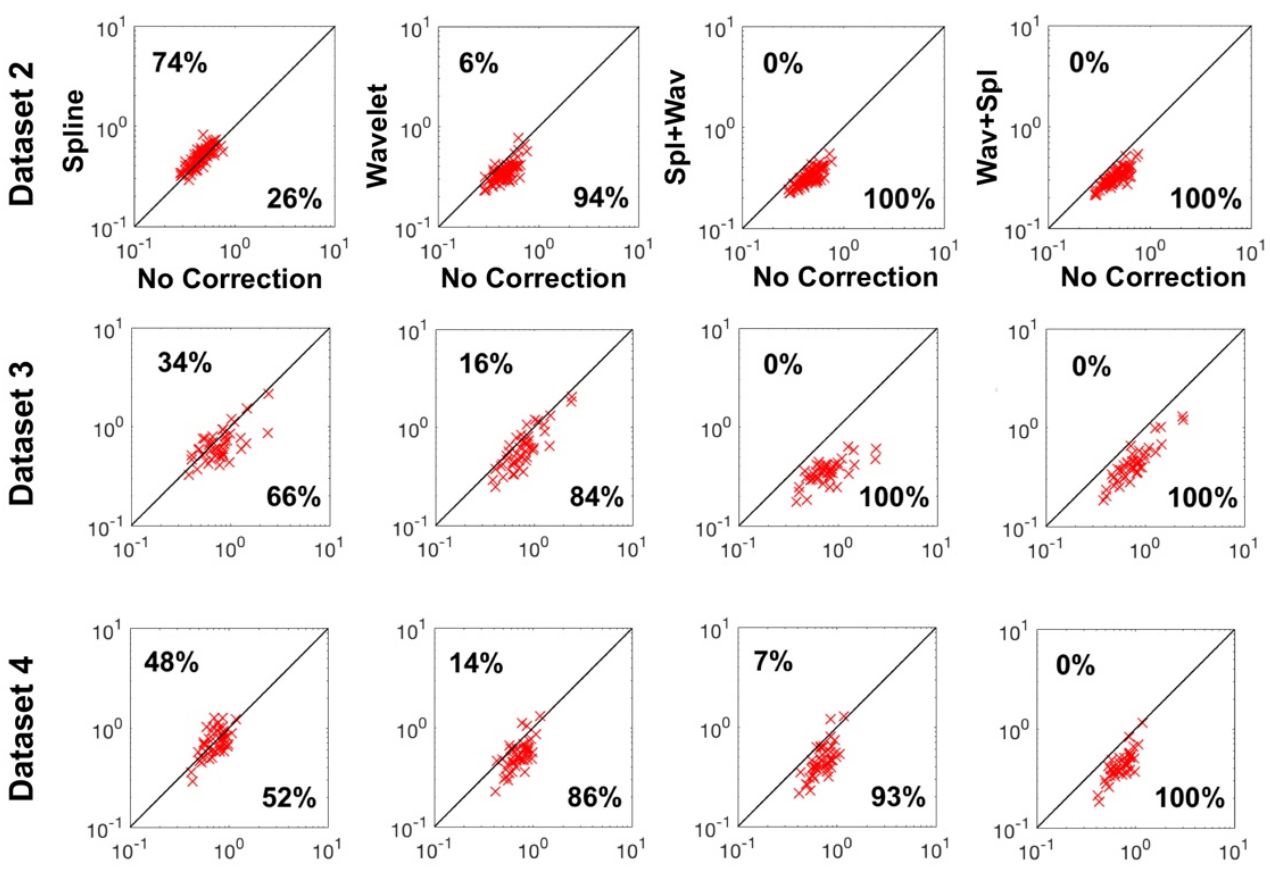

\section{Within-subject SD Correction HbR}
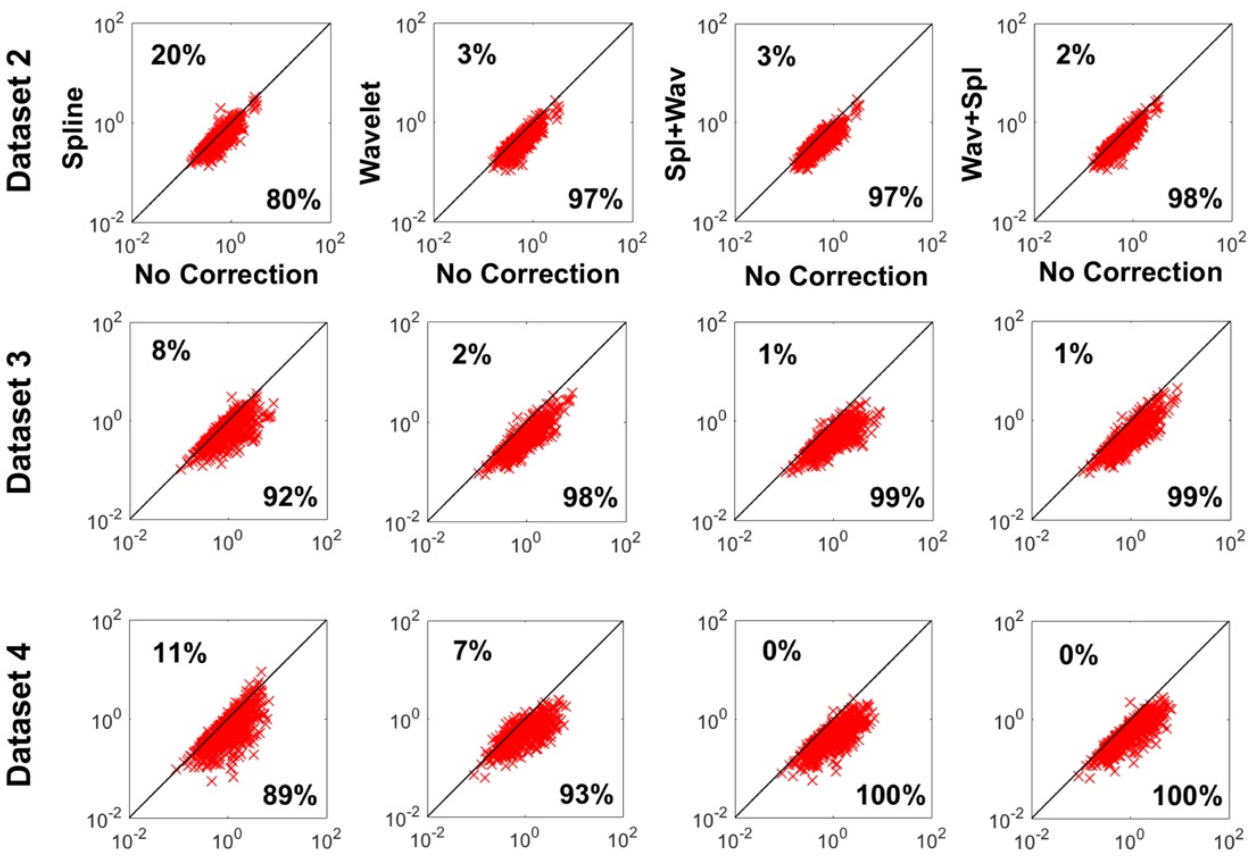

Figure S2.9 Scatter plots of the between-subjects (upper panel) and within-subject (lower panel) SD computed for all the motion correction techniques ( $\mathrm{y}$ axis) versus no motion correction ( $\mathrm{x}$ axis) for $\mathrm{HbR}$ of Datasets 2, 3 and 4 (rows 1, 2, and 3 of each panel). 



\section{Searching for robust infant}

\section{electrophysiological markers of face-sensitivity:}

\section{results from a large longitudinal sample of infants}

Renata Di Lorenzo

Carlijn van den Boomen

Chantal Kemner

Caroline Junge

Under revision for publication

Author contribution:

$\mathrm{CB}, \mathrm{CK}$ and CJ contributed in the conception and design of the study. RDL was responsible for data preprocessing and statistical analyses. RDL and CJ drafted the manuscript. All authors contributed in the revision of the manuscript and approved the submitted version. 


\begin{abstract}
From infancy onwards, EEG is widely used to measure face-sensitivity, that is, differential brain activity to faces vs. non-face stimuli. Three ERP components signal infants' face-sensitivity but reflect possibly different underlying mechanisms: the N290, P400 and the Negative central (Nc). We test whether these are equally robust measures of face-sensitivity across infancy, using a longitudinal dataset of 80 infants tested at five and 10 months. Robust components should manifest itself as a high proportion of 5month-olds showing evidence of face-sensitivity, coupled with a high proportion of those remaining to show this pattern at 10 months, and few showing alternative trajectories. All components show sensitivity to faces already in 5-months-olds, a pattern which did not change over time. Nevertheless, using Markov models we illustrate that the N290 and P400 elicit more robust activations compared to the Nc: a larger proportion of 5-month-olds show the dominant group pattern; a larger proportion of 10-month-olds remain in this dominant group, and a larger proportion of the alternative trajectories from 5- to 10-month-olds move towards the dominant group. We conclude that the N290 and P400 are robust markers to signal face-sensitivity at the individual level.
\end{abstract}




\section{Introduction}

The ability to discriminate human faces from objects, here referred as face-sensitivity, is fundamental for survival. Already in infancy, behavioural research shows that 3-montholds consistently prefer to look at human faces (cf. Libertus, Landa, \& Haworth, 2017). This preference is mirrored in neural measures, such as in event-related potentials (ERPs). To date, numerous studies used ERPs as it is the key method to investigate facesensitivity using the same task (e.g., passively viewing stimuli) across ages. Yet, it remains difficult to understand which ERP components consistently mark (development in) face-sensitivity across infancy. First, most of these infant studies use small sample sizes, which are prone to inflate real effect sizes and make it difficult to reproduce findings (Frank et al., 2017). Second, studies either focus on a single agegroup or use a cross-sectional design, which obscures development (but see Webb, Long, \& Nelson, 2005; Yrttiaho, Forssman, Kaatiala, \& Leppänen, 2014). Third, most studies typically report on a subset of possible ERP components that denote face-sensitivity, which further complicates comparison across studies. The current study therefore reports longitudinal data from 80 infants tested at five and at 10 months using a simple visual contrast: human faces and houses. We aim to test the robustness of all infant ERP components previously defined as face-sensitive.

The literature typically reports three infant ERP-components as sensitive to faces over other categories: The N290, P400 and the Negative central (Nc; de Haan, Johnson, \& Halit, 2003). These components are thought to underlie different mechanisms. The N290 and/or P400 as possible precursors to the adult face-sensitive component N170 have been linked to the perceptual processing of faces (de Haan et al., 2003). In contrast, the Nc is not face-specific but signals face processing as it reflects a child's attention allocation and possibly looking preference (Courchesne, Ganz, \& Norcia, 1981; de Haan \& Nelson, 1997; de Haan et al., 2003). Thus, depending on the contrasting category to which faces are compared, the $\mathrm{Nc}$ might reveal itself as an increase (suggesting increased preferential attention to faces) or as a decrease (suggesting a relative familiarity with faces). 


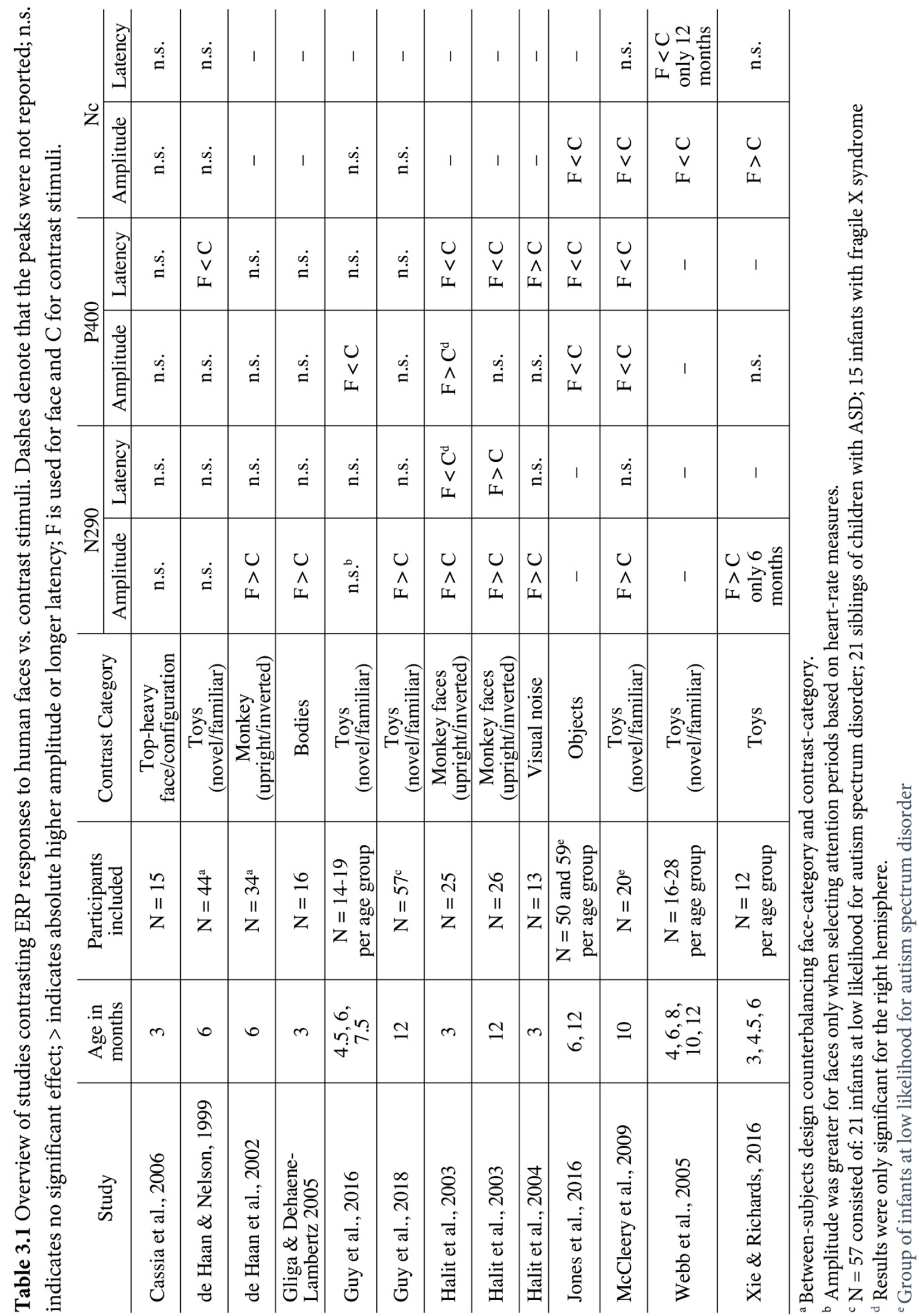


Yet, as reported in Table 3.1 which lists results from previous ERP studies, there is no conclusive evidence on whether any of these components consistently measure facesensitivity across infancy, nor whether they reflect any developmental change in sensitivity across infancy. For instance, while the P400 can be larger for faces (Halit et al., 2003), others report it to be smaller (Jones et al., 2016) or not different from other objects (Guy, Zieber, \& Richards, 2016; Halit, Csibra, Volein, \& Johnson, 2004). Our first aim is therefore to test which of these components prove robust markers of facesensitivity across infancy.

What does robustness entail? First, we expect a robust ERP component of facesensitivity to mirror behavioural evidence. Already newborns, and more consistently, infants older than 3 months, prefer faces over objects (cf. Libertus et al., 2017). The first signs of cortical specialization for faces also appear around the age of three months (Deen et al., 2017; Tzourio-Mazoyer et al., 2002). We therefore expect a robust ERP marker to detect face-sensitivity already at five months, and to remain equally (or increasingly) sensitive at 10 months of age.

Second, although effect sizes can signal the relative strength of the face-sensitivity effect across ages for each component, they do not illuminate the proportion of infants that show the dominant group responses nor the scope of possible trajectories of an individual's response from five to 10 months of age. We therefore use a Markov model approach to illustrate robustness. That is, for each component we quantify the proportion of infants that show a certain pattern of face-sensitivity (e.g. higher amplitudes for faces vs. non-faces) at first measurement and visualize their transition patterns (i.e. from five to 10 months). Note that a Markov Model is purely descriptive. A robust ERP component signalling face-sensitivity should manifest itself as a high proportion of 5-month-olds showing the dominant group pattern, coupled with a high proportion of those remaining to show this pattern at 10 months of age. Likewise, a robust measure of face-sensitivity should further reveal itself as a low proportion of infants showing different responses from the dominant group pattern at five months, and, crucially, by 10 months most of them should show now the same response as the 
majority. This transition to the dominant group could reflect either measurement error at the first visit ('regression to the mean') or those infants having caught up with their peers. However, if it is not a robust measure of face-sensitivity, then there is a variety of transition patterns possible from five months to 10 months, e.g. transitions from one group to other non-dominant groups.

Furthermore, as our second aim we test whether there is development in infants' face-sensitivity from five to 10 months of age. The cross-sectional nature of other infant studies does not allow to draw this conclusion (cf. Table 3.1). Yet there is reason to believe that in the second semester of life a pivotal stage for face processing starts; for instance, they can now categorize faces based on emotion and ethnicity, and discriminate individual faces within their own species (e.g., Anzures, Quinn, Pascalis, Slater, \& Lee, 2010; Leppänen \& Nelson, 2009; Pascalis, de Haan, \& Nelson, 2002). These findings suggest that this leap in development might also be mirrored in basic face processing skill, that is, in face-sensitivity, but evidence is inconclusive. We use ANOVAs to test face-sensitivity changes between the two visits.

To summarize, we use a large longitudinal dataset to advance our understanding of infant face-related ERP components. Our primary goal is to evaluate the robustness of infant face-related components so that future research can start interpreting individual differences in face-sensitivity. Given that infants early in life start orienting to human faces, we hypothesize that the visual-processing components (the N290 and P400) show more consistent patterns than the Nc-component, that indexes attention or preference. We also aim to contribute to the current literature on infant face-sensitivity by testing whether face-sensitive components change between five and 10 months of age. 


\section{Materials and methods}

\section{Participants}

As part of the YOUth cohort (more information on YOUth is available at https://youthonderzoek.nl), we tested 173 healthy 5-month-olds who returned to our center when they were around 10 months. We excluded children because they were born pre-term (<36 weeks; $n=5)$; because after preprocessing their EEG contained less than 10 trials per condition to calculate one of the ERP components $(n=81$; see preprocessing steps below); or due to technical problems $(n=2)$. There were 75 infants who contributed data to all three components at both visits (39 girls, $\mathrm{M}_{\text {age at } 5 \text { months }}=168$, range $=122-214$ days; $M_{\text {age at } 10 \text { months }}=319$, range $=274-368$ days $)$, and an additional 10 infants who contributed data either to the N290 and the P400 components ( $n=5 ; 3$ girls) or to the Nc component ( $\mathrm{n}=5 ; 2$ girls). As a result, all three components comprised data from 80 infants. The study was approved by the medical ethical committee of the University Medical Center Utrecht, in accordance with the Declaration of Helsinki.

\section{Stimuli}

Stimuli were coloured pictures of six female and six male models with a neutral expression selected from the Radboud Faces Database (females identities: 12, 22, 26, 27, 37, 61; males identities: 7, 15, 25, 36, 49, 71; Langner et al., 2010) and 12 coloured pictures of typical Dutch houses selected from the internet. The stimuli were depicted on a grey background (RGB: 108) and measured $20.5 \mathrm{~cm}$ width x $22.5 \mathrm{~cm}$ height (visual angle: $19.4^{\circ} \times 21.2^{\circ}$ ). During the inter-stimulus intervals (ISI) infants saw a $5.3 \times 5.3 \mathrm{~cm}$ square in the middle of the screen, which was composed of four coloured squares (red, yellow, blue and green; visual angle: $4.7^{\circ} \mathrm{x} 4.7^{\circ}$ ).

\section{Procedure}

During the study infants sat on their parent's lap or on a high chair at approximately 65 $\mathrm{cm}$ distance from a 23-inch computer monitor (refresh rate $60 \mathrm{~Hz}, 1920 \mathrm{x} 1080$ resolution). Below the screen there was a webcam camera sampling at $15 \mathrm{~Hz}$ to record 
the child's looking behaviour during the testing session. The testing room was semidark, controlled for luminance (between 8-20 lux) and temperature (between 18-25 ).

During the task infants passively watched trials consisting of pictures of (neutrallylooking) faces or houses. The task was programmed in Matlab using Psych-Toolbox 3 (Brainard \& Vision, 1997). Trial duration was $1000 \mathrm{~ms}$ followed by a jittered ISI between 700 and 1000 ms. In total, there were 96 trials: 48 face trials ( $4 \times 12$ models) and 48 house trials ( 4 x 12 houses). The stimuli order was pseudo-randomized: per block of 24 trials (4 blocks in total) all pictures appeared once in a randomized order. The experimenter played additional sounds or video clips to redirect the child's attention to screen. The experiment ended when all the 96 trials were presented or until the baby was too distracted or fussy to attend. The task lasted approximately 3-4 minutes. Parents were instructed not to interact with their child during the experiment.

\section{ERP recording}

Continuous EEG was recorded at a $2048 \mathrm{~Hz}$ sample rate using a 32-channel ActiveTwo BioSemi system (Actiview version 7.05; Amsterdam, the Netherlands). Electrodes were positioned at standard EEG recording locations according to the international 10-20 system (28 lateral channels: FP1/2; F7/8; F3/4; AF3/4; FC1/2, FC5/6, C3/4, T7/8, CP1/2, $\mathrm{CP} 5 / 6, \mathrm{P} 3 / 4, \mathrm{P} 7 / 8, \mathrm{O} 1 / 2, \mathrm{PO} 3 / 4$; 4 midline channels: Fz, Cz, Pz, Oz). Two electrodes, the Common Mode Sense and the Driven Right Leg, provided the active ground. Electrode offsets were less than $20 \mathrm{nV}$.

\section{Data analyses}

\section{Preprocessing}

EEG data were pre-processed using Brain Vision Analyzer software (version 2.1; Brainproducts, $\mathrm{GmbH}$ ). Data were first down-sampled offline to $512 \mathrm{~Hz}$, and filtered with a high-pass filter of $0.1 \mathrm{~Hz}$ ( $24 \mathrm{~dB} /$ oct), a low-pass filter of $30 \mathrm{~Hz}(24 \mathrm{~dB} / \mathrm{oct})$ and a notch filter of $50 \mathrm{~Hz}$. Continuous EEG data were reduced to epochs of $200 \mathrm{~ms}$ prestimulus until $1000 \mathrm{~ms}$ post-stimulus, with a baseline correction of $-150 \mathrm{~ms}$ to $0 \mathrm{~s}$. We removed whole trials manually when the child looked away from the screen between 0 
and $600 \mathrm{~ms}$ after stimulus onset. Subsequently, we removed trials from single electrodes when an artifact was found between 0-600 ms post-stimulus. Artifacts were defined as amplitudes $+/-200 \mu \mathrm{V}$; as a difference of less than $3 \mu \mathrm{V}$ within a moving window of 200 ms; or as a voltage change of more than $50 \mu \mathrm{V}$ per sampling point. An electrode was rejected if there were less than 5 artifact-free trials. We removed whole trials when more than $16 \%$ of electrodes contained artifacts (based on previous research on face processing in infants, e.g., Halit et al., 2003; van den Boomen, Munsters, \& Kemner, 2019). Finally, we referenced the activity of each single active electrode to the average of all included electrodes before calculating the event-related potential per condition per electrode.

Participants were included in the statistical analyses if the final average per experimental condition contained at least 10 trials for critical electrodes (i.e., for the N290/P400: P3, PO3, O1, Oz, O2, PO4, P4; for the Nc: Fz, C3, C4; cf. Munsters, van Ravenswaaij, van den Boomen, \& Kemner, 2019; van den Boomen et al., 2019). The average number of included segments was 30 per condition (N290/P400: mean 31.6 trials (range 13-47) and 28.8 trials (range 11-47) at five and 10 months, respectively; Nc: mean 31.7 trials (range 13-47) and 28.6 trials (range 11-47) at five and 10 months, respectively).

\section{Component analyses}

Because we are interested in face-sensitivity, that is, the difference between processing faces relative to houses, we required both conditions in our analyses (Luck \& Gaspelin, 2017). For each component ${ }^{1}(\mathrm{~N} 290, \mathrm{P} 400, \mathrm{Nc})$ we chose the mean amplitude rather than latency of each component as our main dependent variable because it is more difficult to assess the latency than amplitude as infant ERPs are characterized by greater slow wave activity resulting in broad peaks in their ERPs (DeBoer, Scott, \& Nelson, 2007), and because mean amplitude is more robust to individual differences in noise effects

\footnotetext{
${ }^{1}$ Note that we did not perform analyses on the P100 component (ERP component related to early visual processing stages) because this peak was not present for the house condition in most infants, and our main focus is on the face-house difference.
} 
that are the result from averaging over different numbers of trials (Luck, 2010). Arguably, one could analyze the N290 peak amplitude, since this is a rather clear-defined peak compared to the other two components. However, since this peak is only present for faces (but not for houses) we could not compare the difference in latency between the two stimulus-types. Supplementary Materials II therefore reports the development of the latency of the $\mathrm{N} 290$ for the face-stimuli, without taking into account the responses to house-stimuli.

For each ERP component, we selected time windows of interest based on previous research on infant face processing. For the N290 this was 170-300 ms (de Haan \& Nelson, 1999), for the P400 300-500 ms (de Haan \& Nelson, 1999), and for the Nc 300$600 \mathrm{~ms}$ (Munsters et al., 2019). We averaged the mean amplitude over all critical electrodes per condition per recording session: for the N290/P400, this was averaged over seven electrodes (P3, PO3, O1, Oz, O2, PO4, P4) and for the Nc over three electrodes (Fz, C3, C4).

\section{Statistical analyses}

For each component we first carried out repeated measures ANOVAs, with mean activity on critical electrodes as our dependent variable, and Stimulus type (faces vs. houses) and Age (five vs. 10 months) as within-subject factors. Note that Supplementary Materials II further repeats these analyses with possible co-variates, as our large sample of infants shows potential variation in certain other subject characteristics relevant to face-sensitivity, which are not of interest to our main research question, but might be worthy of interest to others (i.e., age at visits; time interval between the two visits; and the number of included trials as a proxy for the signal: noise ratio); there we also report for each component the Brown-Forsythe test that indicates similar variation (and therefore noise) of the face-house difference amplitude between the two visits.

Second, we created a Markov model for each component. We formed an index of face-sensitivity for each component for each infant at each age by subtracting the mean amplitude for houses from that of faces. Next, based on the amount and direction of this difference score, we divided the 5-month-olds into three subgroups: no difference 
between faces and houses (between $-/+1.5 \mu \mathrm{V}$ ); a positive difference (i.e., faces vs. houses elicited a larger than $1.5 \mu \mathrm{V}$ positive response); or a negative difference (i.e., faces vs. houses elicited a larger than $1.5 \mu \mathrm{V}$ negative response). The second author -blind to the results of the current study- defined the thresholds by reviewing the observed differences in previous research on face-sensitivity (Halit et al., 2003; Munsters et al., 2019; van den Boomen et al, 2019; Webb et al., 2005). Note that these thresholds are arbitrary and open to discussion, and thus should not be considered absolute but rather be used as an indication of meaningful differences. Once we categorized the 5-montholds into separate subgroups, we calculated for each component the transitional probabilities of a child either remaining in the same group or moving to one of the other two groups at 10 months (using the same thresholds).

\section{Results}

\section{N290 amplitude}

Figure 3.1 depicts the ERPs at posterior-occipital electrodes, time-locked to the onset of faces and houses for the 5-month-olds and 10-month-olds. For the N290, the repeated measures ANOVA shows that there is a main effect of Stimulus type $(F(1,79)=209, p<$ $.0001, \eta^{2}=.73$ ): for both ages, faces elicited a less positive N290 (i.e., more negative; $M$ $=7.31 \mu \mathrm{V}, S D=6.91)$ than houses $(M=16.6 \mu \mathrm{V}, S D=6.81)$. There is also a main effect of Age $\left(F(1,79)=16.0, p<.0001, \eta^{2}=.17\right)$, with 5 -month-olds showing a smaller positive mean amplitude across both stimulus types $(M=9.60 \mu \mathrm{V}, S D=7.28)$ than 10 -montholds $(M=14.3 \mu \mathrm{V}, S D=8.90)$. There is no interaction between Age and Stimulus type $\left(F(1,79)=1.60, p=.21, \eta^{2}=.020\right)$. 


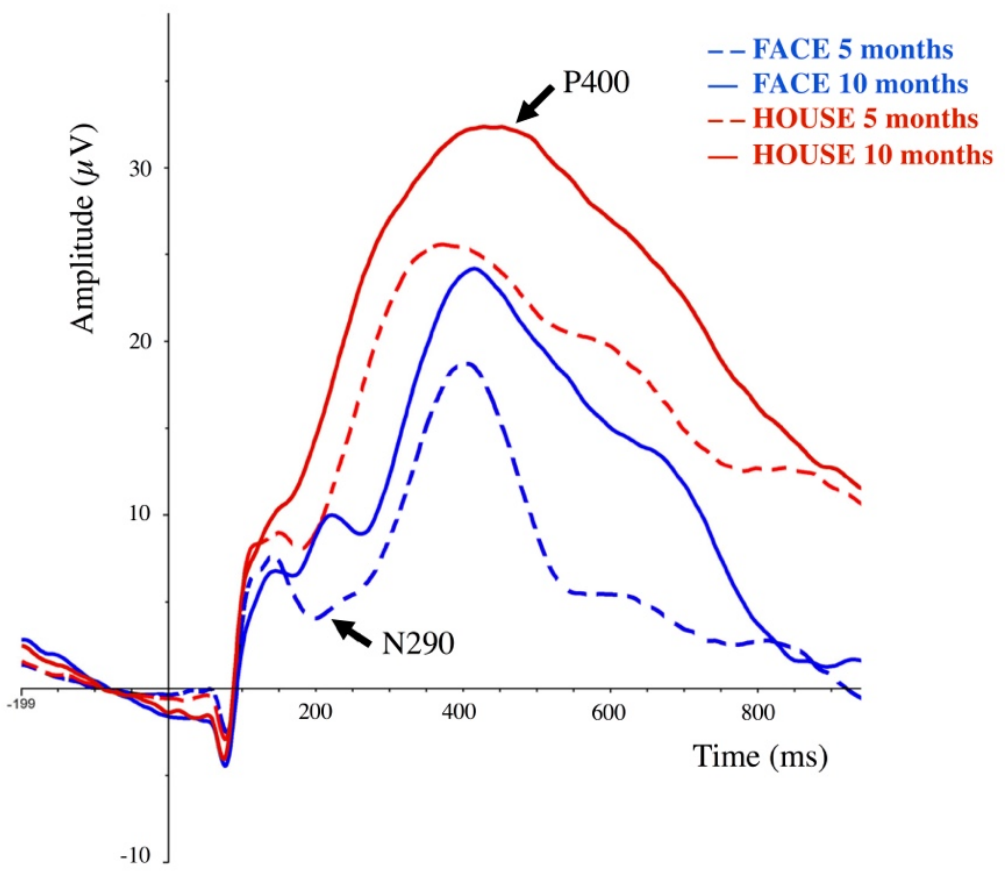

Figure 3.1 Grand-averaged waveforms obtained at five (dashed lines) and 10 months (solid lines) of age in response to faces (blue lines) and houses (red lines). The plot represents the mean activity recorded from electrodes $\mathrm{O} 1, \mathrm{O} 2, \mathrm{Oz}, \mathrm{P} 3, \mathrm{PO} 3, \mathrm{P} 4$, and $\mathrm{PO} 4$.

Figure 3.2 plots the Markov model for the N290. The majority of our infants ( $\mathrm{n}=$ $58 ; 72.5 \%$ of the sample) shows a negative face-house difference at both ages. For those 14 5-month-olds who show either a positive or no-difference effect, all but one has moved to the negative-difference group as 10-month-olds. Strikingly, not all trajectories are present out of the possible trajectories; for instance, there is not one child moving from a positive face-house difference at visit 1 to a neutral difference at visit 2, which is what one would expect to occur if development is gradual, that is, from a positive difference shifting towards a negative difference.

Table 3.2 reports the mean values for each of the subgroups and the range, for the 5month-olds and 10-month-olds, respectively, for all three components of interest. 


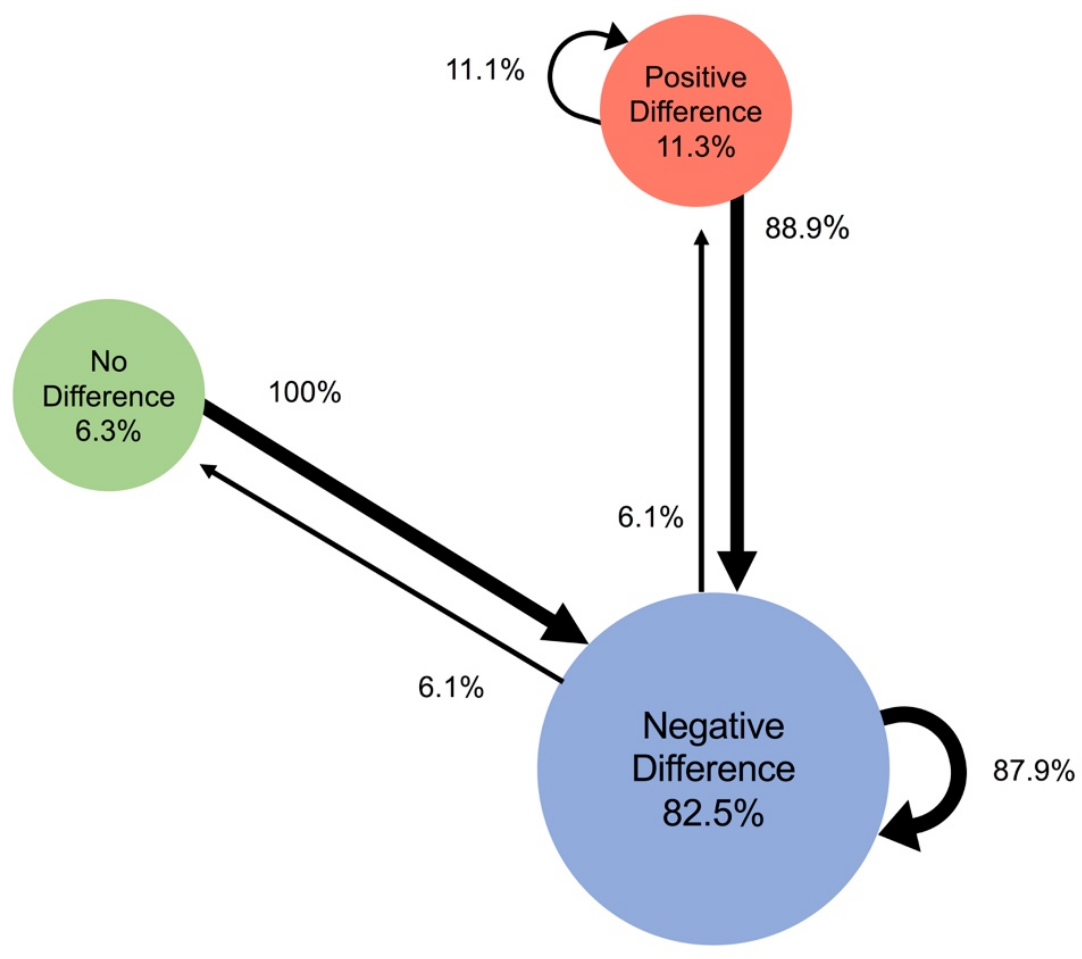

Figure 3.2 Markov model depicting the transition trajectories observed from five to 10 months for the N290 amplitude denoting face-sensitivity. Circles report the percentage of infants that at five months show either no difference between face-house amplitudes (green), a negative difference (blue), or a positive difference (red). Circular arrows indicate the percentage of infants that at 10 months remain in a category, while straight arrows indicate the percentage of five-month-olds that moved from one category to another at 10 months of age.

Table 3.2 Descriptive subgroup summaries for each of the three components denoting face-sensitivity (face-house amplitude), split by age. Note: All values are in $\mu \mathrm{V}$ and are based on the difference in mean amplitude of the ERP component for face minus houses; $\mathrm{n}=$ subgroup size, Min. = minimum, Max. = maximum, $\mathrm{SD}=$ standard deviation.

\begin{tabular}{l|ccccc|ccccc} 
& \multicolumn{7}{|c|}{5 months } & \multicolumn{5}{c}{ 10 months } \\
\hline N290 & $\mathbf{n}$ & Mean & Min. & Max. & SD & n & Mean & Min. & Max. & SD \\
\hline Negative difference & 66 & -10.94 & -27.06 & -1.73 & 6.22 & 71 & -12.00 & -35.08 & -2.15 & 7.53 \\
No difference & 5 & 0.19 & -0.45 & 1.01 & 0.63 & 4 & -0.07 & -0.57 & 1.24 & 0.87 \\
Positive difference & 9 & 5.80 & 2.46 & 10.54 & 2.53 & 5 & 7.97 & 2.20 & 17.88 & 5.89 \\
\hline P400 & & & & & & & & & & \\
\hline Negative difference & 68 & -11.87 & -26.85 & -1.60 & 6.59 & 66 & -12.97 & -31.12 & -2.73 & 6.46 \\
No difference & 5 & -0.40 & -1.39 & 1.13 & 1.05 & 7 & 0.22 & -1.41 & 1.39 & 1.12 \\
Positive difference & 7 & 7.10 & 3.66 & 10.90 & 3.08 & 7 & 5.83 & 1.58 & 12.34 & 4.13 \\
\hline Nc & & & & & & & & & & \\
\hline Negative difference & 14 & -4.16 & -14.27 & -1.51 & 3.32 & 14 & -6.93 & -25.91 & -2.03 & 6.12 \\
No difference & 18 & 0.16 & -1.40 & 1.36 & 0.93 & 17 & 0.09 & -1.36 & 1.38 & 0.91 \\
Positive difference & 48 & 6.17 & 1.51 & 19.63 & 4.42 & 49 & 6.19 & 1.55 & 13.62 & 2.92 \\
\hline
\end{tabular}




\section{P400 amplitude}

For the P400 amplitude (also visible in Figure 3.1), the repeated measures ANOVA shows that there is again a main effect of Stimulus type $\left(F(1,79)=207, p<.0001, \eta^{2}=\right.$ .72 , with houses eliciting a more positive amplitude $(M=26.8 \mu \mathrm{V}, S D=8.13)$ than faces $(M=17.0 \mu \mathrm{V}, S D=8.98)$. There is also a main effect of Age $(F(1,79)=31.7, p<.0001$, $\left.\eta^{2}=.29\right)$ : the $\mathrm{P} 400$ mean amplitude increased significantly from five $(M=17.9 \mu \mathrm{V}, S D$ $=9.53)$ to 10 months $(M=25.9 \mu \mathrm{V}, S D=10.9)$. There is no interaction between Age and Stimulus type $\left(F(1,79)=0.26, p=.61, \eta^{2}=.003\right)$.

Figure 3.3 plots the Markov model for the P400 amplitude. Results show that the dominant group pattern is a negative difference (that is, faces elicit a less positive P400 than houses): there are 58 children (72.5\%) who show this pattern both at five and 10 months. The remaining children show nearly all possible patterns, although again, out of the 12 infants not showing a negative difference at five months, the majority $(n=8$; $67 \%)$ returns to the group dominant response at 10 months.

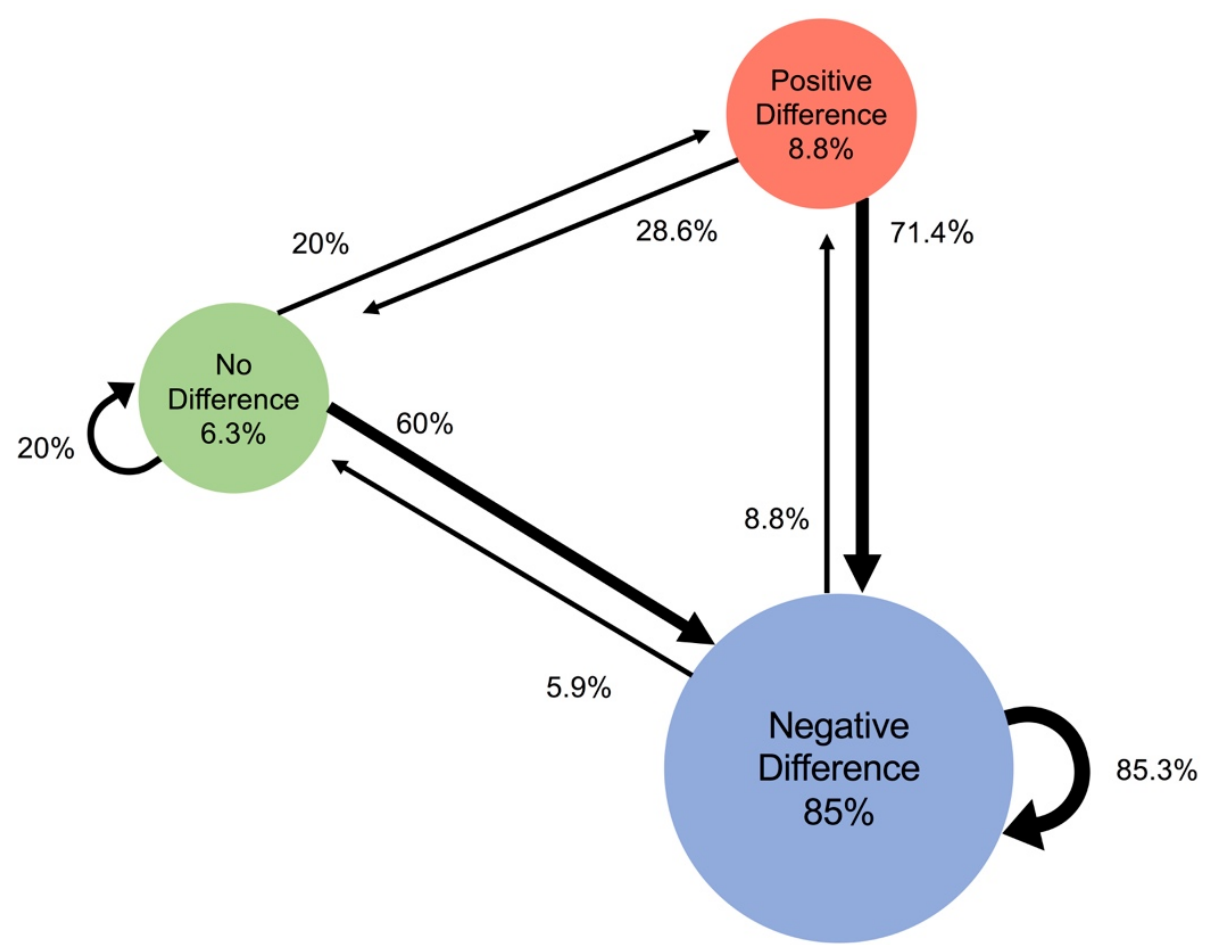

Figure 3.3 Markov model depicting the transition trajectories observed from five to 10 months for the P400 amplitude denoting face-sensitivity. Circles report the percentage of infants that at five months show either no difference between face-house amplitudes (green), a negative difference (blue), or a positive difference (red). The circular arrows indicate the percentage of infants that at 10 months remain 
in a category, while straight arrows indicate the percentage of 5-month-olds that moved from one category to another at 10 months of age.

\section{Nc amplitude}

Figure 3.4 plots the ERPs at fronto-central electrodes, time-locked to the onset of faces and houses for the 5-month-olds and 10-month-olds. For the Nc, the repeated measures ANOVA shows that there is again a main effect of Stimulus type $(F(1,79)=33.6, p<$ $\left..0001, \eta^{2}=.30\right)$ : for both ages, faces elicited a smaller $\mathrm{Nc}(M=-8.50 \mu \mathrm{V}, S D=4.60)$ than houses $(M=-11.3 \mu \mathrm{V}, S D=4.31)$. There is also a main effect of Age $(F(1,79)=22.4, p<$ $\left..0001, \eta^{2}=.22\right)$, with 5 -month-olds showing a smaller Nc across both stimulus types $(M$ $=-8.05 \mu \mathrm{V}, S D=4.45)$ than 10 -month-olds $(M=-11.7 \mu \mathrm{V}, S D=5.91)$. There is no interaction between Age and Stimulus type $\left(F(1,79)=0.23, p=.63, \eta^{2}=.003\right)$.

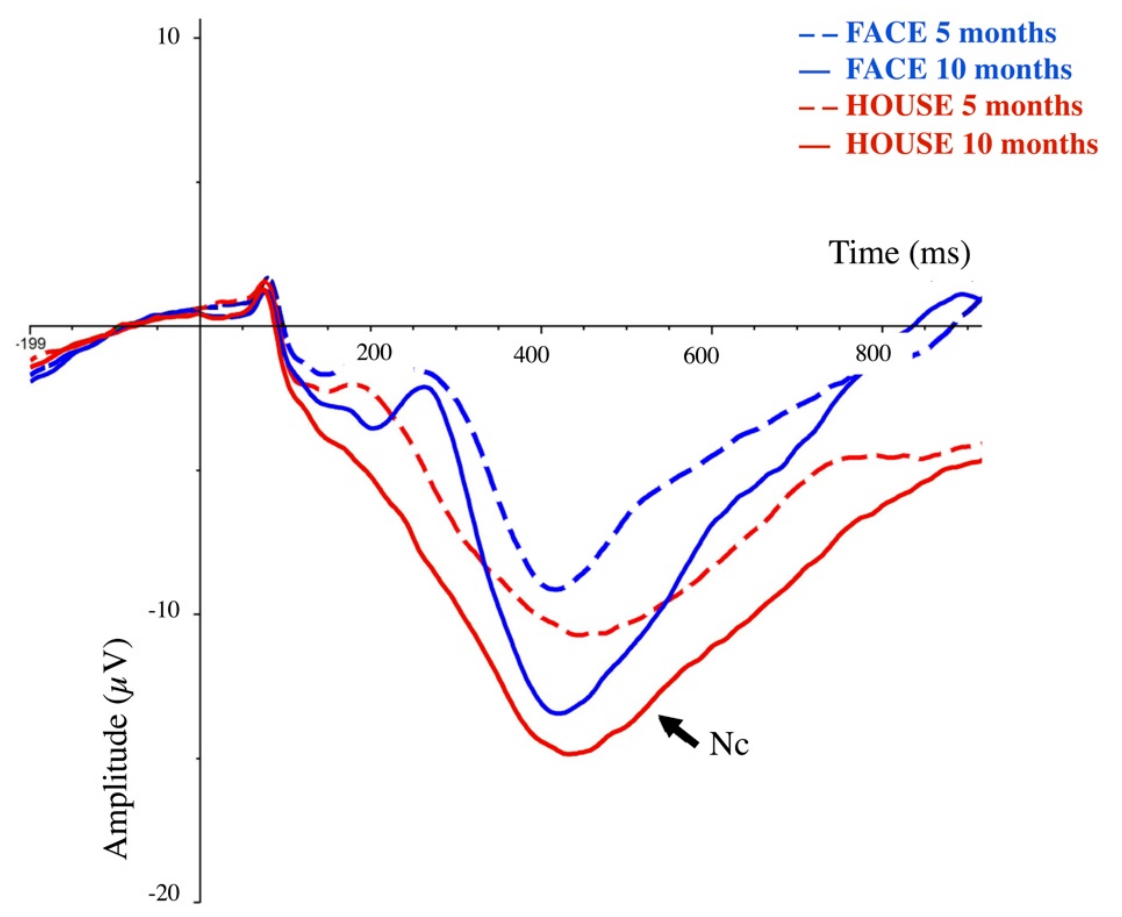

Figure 3.4 Grand-averaged waveforms obtained at five (dashed lines) and at 10 months (solid lines) of age in response to faces (blue lines) and houses (red lines). The plot represents the mean activity recorded from fronto-central electrodes Fz, C3, C4.

Figure 3.5 illustrates the Markov model for the Nc. For this component, the dominant group response at both ages is a positive difference (i.e., corresponding to a smaller Nc for faces than for houses). However, compared to the two previous 
components, fewer children show the dominant group response at both measurements (e.g., $\mathrm{n}=32$, that is, $40 \%$ of total sample). Moreover, all possible trajectories appear now to be possible, with higher rates of the non-dominant trajectories. For instance, 16 out of the 48 children who showed the dominant pattern at five months (33.3\%) no longer shows this pattern at 10 months. Out of the 32 infants who did not demonstrate the dominant group response at five months, only 17 infants (53\%) regress to the dominant group response at 10 months.

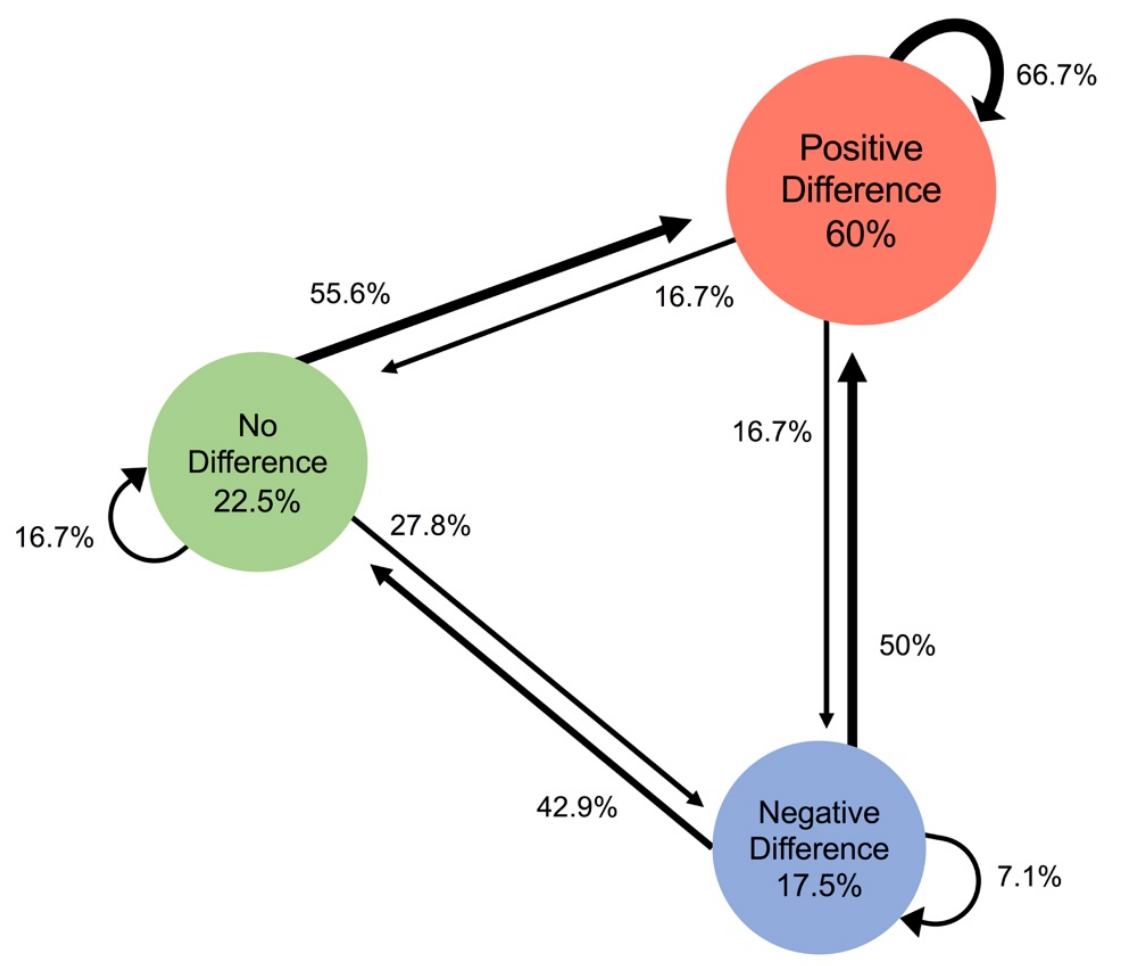

Figure 3.5 Markov model depicting the transition trajectories observed from five to 10 months for the Nc amplitude denoting face-sensitivity. Circles report the percentage of infants that at five months show either no difference between face-house amplitudes (green), a negative difference (blue), or a positive difference (red). The circular arrows indicate the percentage of infants that at 10 months remain in a category, while straight arrows indicate the percentage of 5-month-olds that moved from one category to another at 10 months of age. 


\section{Discussion}

In the current study we used longitudinal ERP data of 80 infants who came to our lab twice within a 5-month-interval to participate in a face-discrimination task. At both ages we observe the components typically reported for face-sensitivity in infant studies: the N290, the P400 and the Negative central. At a group level, the N290 and P400 were more negative when evoked by faces compared to houses, whereas the opposite pattern was found for the Nc. However, not all components proved equally robust. By comparing the components on the proportion of infants that followed the dominant group pattern at either five or at 10 months, we show that for the visual components N290/P400 nearly twice as many infants belong to the dominant group - and remain doing so at 10 months - than was the case for the attentional Nc. Our second finding is that at a group level there is no development in face-sensitivity for any of the components between the first and second semester of life. Below we discuss these findings in turn.

Our first aim was to compare the robustness of the three infant components of face-sensitivity. The ANOVA analyses suggest that at the group level they can all be considered indices of face-sensitivity as they all show main effects of Stimulus type. However, it is in the Markov models that we observe clear differences between our three components of interest in face-sensitivity. The Markov model for the N290 patterns like the Markov model for the P400, but the Markov model for the Nc component is strikingly different, in three ways. First, while in both cases there is a clear dominant group response at five months, the groups markedly differ in size: the dominant N290/P400 response comprises more cases (83-85\%) compared to the dominant Nc group response (60\%). Second, at 10 months, there are more infants who remain in the dominant group for the $\mathrm{N} 290 / \mathrm{P} 400$ (88\% and $85 \%$ respectively) than for the $\mathrm{Nc}(67 \%)$. Finally, there are fewer types of transitions between groups for the N290/P400 than for the Nc. Crucially, the dominant trajectory for those 5-month-olds in the non-dominant groups for the N290/P400 is towards the dominant group (93\% and 67\%, respectively), whereas this trend is less apparent for the Nc (53\%). Together, these findings suggest that the N290/P400 components are more robust than the Nc. 
Can we really conclude that the N290/P400 components prove more robust? Recall that for each component we used the same a-priori defined threshold to calculate groupmembership; consequently, it remains possible that results change had we chosen different thresholds across components, or selected individual thresholds for each of the components. Nevertheless, our findings are in line with a recent infant ERP study showing that face-sensitive components differ in test-retest reliability: While test-retest reliability is substantial for the $\mathrm{N} 290$, it is only moderate for the Nc component (Munsters et al., 2019). In short, our findings strongly suggest that the N290/P400 patterns differ from the Nc in terms of robustness.

We speculate that the difference in robustness among the components might relate to the nature of their underlying mechanisms. The N290 and the P400 are ERP components often associated with the early stages of visual processing of faces (de Haan et al., 2003; Nelson \& McCleery, 2008). It is possible that the early and extensive exposure to faces that neonates experience swiftly contributes in making the sensitivity of these visual components to emerge early and remain stable over time. As ERPs mainly reflect activation from the cortex (Luck, 2014), this reasoning is in line with a current developmental view on face processing. Johnson (2015) suggests that cortical specialization emerges as the result of infants' increased experience with faces, while it is also influenced by intrinsic biases to orient to faces and mechanisms of inter-regional connectivity.

While the N290/P400 are related to perceptual processing, it is likely that the Nc subserves a different mechanism. Recall that the direction of the Nc remains difficult to predict as it is thought to reflect attention allocation, preference or some aspects of recognition (de Haan et al., 2003; Nelson \& McCleery, 2008). All these processes associated with the Nc-component reflect higher-level processes of attention, which possibly compete with each other over the course of the experiment and across development. In other words, the Nc might not only pick up on the general face-house contrast, but might also fluctuate as it is sensitive to familiarity-novelty at the item-level. 
Consequently, the underlying mechanisms of the Nc may be less steady over time or across individuals compared to the visual processing indexed by N290/P400.

Compared to the Nc, the visual components N290 and the P400 pattern alike in face-sensitivity: both show a larger negativity (that is, reduced positivity) for faces compared to houses. Likewise, in adults and older children, the amplitude of the facesensitive N170 has been found to be more negative in response to faces over other objects (e.g., Kuefner, De Heering, Jacques, Palmero-Soler, \& Rossion, 2010). There is debate in the infant literature whether the N290/P400 complex together serve as a precursor to the adult N290 (e.g. Halit et al., 2004), or whether both resemble distinct processes in face-sensitivity. While there is general consensus that the infant N290 is a precursor to the adult N170, less is clear about the interpretation of the P400. Part of the confusion about the interpretation of the P400 possibly stems from mixed findings concerning the polarity of the P400: The literature documents both positive (Halit et al., 2003) as well as negative differences (Guy et al., 2016; Jones et al., 2016; cf. Table 3.1). For instance, one study argues that it is only the N290 that is related to the N170, as cortical source analyses suggest similar brain regions involved in the N170 and N290 (e.g., the fusiform gyrus); in contrast, the generators involved in the P400 were not different from those involved in the Nc, which suggests that the P400 is also influenced by the activity of the (positive) dipole sources generating the Nc (Guy et al., 2016). If this were true one would expect the Markov model of the P400 to resemble more closely that of the Nc; instead we observe it patterns more like the N290, albeit not completely (e.g., not in the number of possible trajectories). More longitudinal research with older children is required to advance our understanding of the P400. For now, we conclude that our results further underscore that the N290 is a precursor to the adult N170, given its similarity in polarity.

Our second aim focused on understanding whether the face-related components reflect developmental changes in face-sensitivity between five and 10 months of age. For each component, the ANOVAs revealed that there is no change in sensitivity over time. This lack of change for processing faces vs. houses was not related to differences in the 
infants' age at the first visit, nor to differences in the time interval between the two visits. Our results are consistent with a previous study reporting that the N170 maintains a similar sensitivity to faces in children between four and 17 years of age (Kuefner et al., 2010).

Although we did not observe any development in face-sensitivity, infants' cortical activity increased in amplitude from five to 10 months of age. These developmental effects are in line with previous research suggesting that the developmental changes of certain ERP components might follow a U-shaped function from infancy to childhood: The amplitude increases throughout infancy before it decreases again in childhood (de Haan, 2007). Also, such amplitude changes have been previously linked to changes in synaptic density (Courchesne, 1990; Vaughan \& Kurtzberg, 1992). Indeed, the infant brain undergoes substantial functional and structural changes during the first year of life: between four and six months of age there is a burst of synapse formation in the visual cortex, and around eight and 12 months of age there is the emergence of white matter in frontal, parietal and occipital regions (Johnson, 2001). Therefore, the amplitude changes between five and 10 months in our study might simply indicate a general increase in synaptic density and brain activity towards visual stimuli. Similar increases in amplitude have also been reported in one longitudinal study that tested the amplitude maturation of the Nc component in 4- to 12-month-olds (Webb et al., 2005). In contrast, a cross-sectional study that targeted 4.5-, 6- and 7.5-month-old infants (Guy et al., 2016) does not report such increases in amplitude. It is possible that observing such amplitude increases requires a wider age range that covers five to 10 months. Another possibility is that such increases only become apparent in larger longitudinal samples as in ours, because infants might vary substantially in their neuro-development (Johnson, 2001). In any case, our study with 80 infants at both five and 10 months reveals a general increase in cortical activity related to visual processing.

In summary, our findings indicate that compared to the Nc, the N290/P400 complex are more robust markers of face-sensitivity between five and 10 months of age. This finding suggests a difference in the underlying mechanisms. In addition, we 
showed that all three components do not show any change in face-sensitivity in 5- to 10month-old infants, which indicates that sensitivity to faces remains similar between these ages.

This work is meant as the first assessment of a larger dataset which aims to investigate how individual differences in face-sensitivity are linked to differences in the development of social cognition or social competence. Given that the N290/P400 appear more robust, we suggest that it is worthwhile to track development of those children that did not follow the dominant group patterns for the N290/P400 in infancy. Nevertheless, studies aiming to grasp individual differences usually require outcomes with a maximum of between-participant variability rather than robust findings at a group level (Hedge, Powell, \& Sumner, 2018). It is therefore also possible that it is individual variation in the Nc rather than in the $\mathrm{N} 290 / \mathrm{P} 400$ that proves meaningful in explaining subsequent development. It therefore remains to be seen whether it is actually in the domain of perceptual processing (i.e., N290/P400; Jones et al., 2016, 2017; McCleery et al., 2009) or in the domain of attention and preference (Nc; Jones et al., 2016) that we find such meaningful individual differences in face-sensitivity.

\section{Acknowledgements}

This research was financed through YOUth. YOUth is an initiative of Utrecht University and UMC Utrecht. YOUth is part of (and partly funded by) the research theme Dynamics of YOUth of Utrecht University and UMC Utrecht Brain Center. YOUth is also funded through the Gravitation program of the Dutch Ministry of Education, Culture, and Science and the Netherlands Organisation for Scientific Research (NOW grant number 024.001.003). The funders had no role in study design, data collection and analysis, decision to publish, or preparation of the manuscript. We thank all infants and their parents who participated for making this study possible; thank Roy van Koten who created the script and wrote the program to code infant looking behavior. We also express thanks to all testing assistants at the Child Research Center at Utrecht University 
Chapter 3

for their help in testing infants, and Charlotte Blom, Elles Burg, and Nikki van der Heijden helping with the preprocessing of the data.

RDL was supported by a grant from the European Community's Horizon 2020 Program under grant agreement nº 642996 (Brainview). 


\section{References}

Anzures, G., Quinn, P. C., Pascalis, O., Slater, A. M., \& Lee, K. (2010). Categorization, categorical perception, and asymmetry in infants' representation of face race. Developmental Science, 13(4), 553-564.

Brainard, D., \& Vision, S. (1997). The psychophysics toolbox. Spatial vision, 10, 433-436. https://doi.org/10.1163/156856897x00357

Cassia, V. M., Kuefner, D., Westerlund, A., \& Nelson, C. A. (2006). A behavioural and ERP investigation of 3-month-olds' face preferences. Neuropsychologia, 44(11), 2113-2125.

Courchesne, E. (1990). Chronology of postnatal human brain development: Event-related potential, positron emission tomography, myelinogenesis, and synaptogenesis studies. In: J.W. Rohrbaugh, R. Parasuraman, \& R. Johnson Jr. (Eds.), Event related brain potentials: Basic issues and applications. New York: Oxford University Press, pp. 210241

Courchesne, E., Ganz, L., \& Norcia, A. M. (1981). Event-related brain potentials to human faces in infants. Child development, 804-811. https://doi.org/10.2307/1129080

de Haan, M. (2007). Current and future directions in infant electrophysiology. In: de Haan M. (Ed.), Infant EEG and event-related potentials, Psychology Press, New York, pp. 305314 .

de Haan, M., \& Nelson, C. A. (1997). Recognition of the mother's face by six-month-old infants: A neurobehavioral study. Child development, 68(2), 187-210.

de Haan, M., \& Nelson, C. A. (1999). Brain activity differentiates face and object processing in 6-month-old infants. Developmental psychology, 35(4), 1113.

de Haan, M., Johnson, M. H., \& Halit, H. (2003). Development of face-sensitive event-related potentials during infancy: a review. International Journal of Psychophysiology, 51(1), $45-$ 58. https://doi.org/10.1016/S0167-8760(03)00152-1

de Haan, M., Pascalis, O., \& Johnson, M. H. (2002). Specialization of neural mechanisms underlying face recognition in human infants. Journal of Cognitive Neuroscience, 14(2), 199-209.

DeBoer, T., Scott, L. S., \& Nelson, C. A. (2007). Methods for acquiring and analyzing infant event-related potentials. In de Haan M. (Ed.), Infant EEG and event-related potentials, (pp. 5-37). New York, NY, US: Psychology Press.

Deen, B., Richardson, H., Dilks, D. D., Takahashi, A., Keil, B., Wald, L. L., ... \& Saxe, R. (2017). Organization of high-level visual cortex in human infants. Nature Communications, 8 , 13995. 
Frank, M. C., Bergelson, E., Bergmann, C., Cristia, A., Floccia, C., Gervain, J., ... \& LewWilliams, C. (2017). A collaborative approach to infant research: Promoting reproducibility, best practices, and theory-building. Infancy, 22(4), 421-435. https://doi.org/10.1111/infa.12182

Gliga, T., \& Dehaene-Lambertz, G. (2005). Structural encoding of body and face in human infants and adults. Journal of Cognitive Neuroscience, 17(8), 1328-1340.

Guy, M. W., Richards, J. E., Tonnsen, B. L., \& Roberts, J. E. (2018). Neural correlates of face processing in etiologically-distinct 12-month-old infants at high-risk of autism spectrum disorder. Developmental cognitive neuroscience, 29, 61-71. https://doi.org/10.1016/j.dcn.2017.03.002

Guy, M. W., Zieber, N., \& Richards, J. E. (2016). The cortical development of specialized face processing in infancy. Child development, 87(5), 1581-1600.

https://doi.org/10.1111/cdev.12543

Halit, H., Csibra, G., Volein, A., \& Johnson, M. H. (2004). Face-sensitive cortical processing in early infancy. Journal of Child Psychology and Psychiatry, 45(7), 1228-1234. https://doi.org/0.1111/j.1469-7610.2004.00321.x

Halit, H., de Haan, M., \& Johnson, M. H. (2003). Cortical specialisation for face processing: face-sensitive event-related potential components in 3-and 12-month-old infants. NeuroImage, 19(3), 1180-1193. https://doi.org/10.1016/S1053-8119(03)00076-4

Hedge, C., Powell, G., \& Sumner, P. (2018). The reliability paradox: Why robust cognitive tasks do not produce reliable individual differences. Behavior Research Methods, 50(3), 1166-1186.

Johnson, M. H. (2001). Functional brain development in humans. Nature Reviews Neuroscience, 2(7), 475.

Johnson, M. H., Senju, A., \& Tomalski, P. (2015). The two-process theory of face processing: modifications based on two decades of data from infants and adults. Neuroscience \& Biobehavioral Reviews, 50, 169-179. https://doi.org/10.1016/j.neubiorev.2014.10.009

Jones, E. J., Venema, K., Earl, R., Lowy, R., Barnes, K., Estes, A., ... \& Webb, S. J. (2016). Reduced engagement with social stimuli in 6-month-old infants with later autism spectrum disorder: a longitudinal prospective study of infants at high familial risk. Journal of Neurodevelopmental Disorders, 8(1), 7. https://doi.org/10.1186/s11689-0169139-8

Jones, E. J., Venema, K., Earl, R. K., Lowy, R., \& Webb, S. J. (2017). Infant social attention: an endophenotype of ASD-related traits?. Journal of Child Psychology and Psychiatry, 58(3), 270-281. https://doi.org/10.1111/jcpp.12650 
Kuefner, D., De Heering, A., Jacques, C., Palmero-Soler, E., \& Rossion, B. (2010). Early visually evoked electrophysiological responses over the human brain (P1, N170) show stable patterns of face-sensitivity from 4 years to adulthood. Frontiers in Human Neuroscience, 3, 67.

Leppänen, J. M., \& Nelson, C. A. (2009). Tuning the developing brain to social signals of emotions. Nature Reviews Neuroscience, 10(1), 37.

Libertus, K., Landa, R. J., \& Haworth, J. L. (2017). Development of Attention to Faces during the First 3 Years: Influences of Stimulus Type. Frontiers in Psychology, 8, 1976.

Luck, S. J. (2010). Is it legitimate to compare conditions with different numbers of trials? Retrieved from http://www.erpinfo.org/uploads/5/8/4/6/58469631/mean_peak_noise.pdf

Luck, S. J. (2014). An Introduction to the Event-Related Potential Technique. MIT Press.

Luck, S. J. \& Gaspelin, N. (2017), How to get statistically significant effects in any ERP experiment (and why you shouldn't). Psychophysiology, 54, 146-157. https://doi.org/10.1111/psyp.12639.

McCleery, J. P., Akshoomoff, N., Dobkins, K. R., \& Carver, L. J. (2009). Atypical face versus object processing and hemispheric asymmetries in 10-month-old infants at risk for autism. Biological Psychiatry, 66(10), 950-957.

https://doi.org/10.1016/j.biopsych.2009.07.031

Munsters, N.M., van Ravenswaaij, H., van den Boomen, C., \& Kemner, C. (2019). Test- retest reliability of infant event related potentials evoked by faces. Neuropsychologia, 126, 2026. http://doi.org/ 10.1016/j.neuropsychologia.2017.03.030

Nelson, C. A., \& McCleery, J. P. (2008). Use of event-related potentials in the study of typical and atypical development. Journal of the American Academy of Child \& Adolescent Psychiatry, 47(11), 1252-1261. https://doi.org/10.1097/CHI.0b013e318185a6d8.

Pascalis, O., de Haan, M., \& Nelson, C. A. (2002). Is face processing species-specific during the first year of life?. Science, 296(5571), 1321-1323. https://doi.org/10.1126/science.1070223

Tzourio-Mazoyer, N., De Schonen, S., Crivello, F., Reutter, B., Aujard, Y., \& Mazoyer, B. (2002). Neural correlates of woman face processing by 2-month-old infants. Neuroimage, 15(2), 454-461.

van den Boomen, C., Munsters, N., \& Kemner, C. (2019). Emotion processing in the infant brain: The importance of local information. Neuropsychologia, 126, 62-68. https://doi.org/10.1016/j.neuropsychologia.2017.09.006 
Chapter 3

Vaughan, H. G. Jr., \& Kurtzberg, D. (1992). Electrophysiological indices of human brain maturation and cognitive development. In: M.R. Gunnar \& C.A. Nelson (Eds.), Minnesota Symposia on Child Psychology Vol. 24. Hillsdale, NJ: Lawrence Erlbaum Associate Inc., pp. 1-36

Webb, S. J., Long, J. D., \& Nelson, C. A. (2005). A longitudinal investigation of visual eventrelated potentials in the first year of life. Developmental Science, 8(6), 605-616. https://doi.org/10.1111/j.1467-7687.2005.00452.x

Xie, W., \& Richards, J. E. (2016). Effects of interstimulus intervals on behavioral, heart rate, and event-related potential indices of infant engagement and sustained attention. Psychophysiology, 53(8), 1128-1142. https://doi.org/10.1111/psyp.12670

Yrttiaho, S., Forssman, L., Kaatiala, J., \& Leppänen, J. M. (2014). Developmental precursors of social brain networks: the emergence of attentional and cortical sensitivity to facial expressions in 5 to 7 months old infants. PloS one, 9(6), e100811. https://doi.org/0.1371/journal.pone.0100811 


\section{Supplementary Materials II}

\section{N290 Latency (face stimuli)}

Figure S3.1 (left panel) shows the boxplots of N290 latency recorded in response to faces at five and 10 months, (right panel) depicts the scatterplot between N290 latency to faces at five and at 10 months. The paired sample t-test computed to test differences in N290 peak latency in response to faces over time revealed a shorter latency at five months ( $M$ $=234 ; S D=19.9)$ compared to that at 10 months $(M=240 ; S D=21.3), t(79)=-2.05, p$ $=.044, d=-.23$.
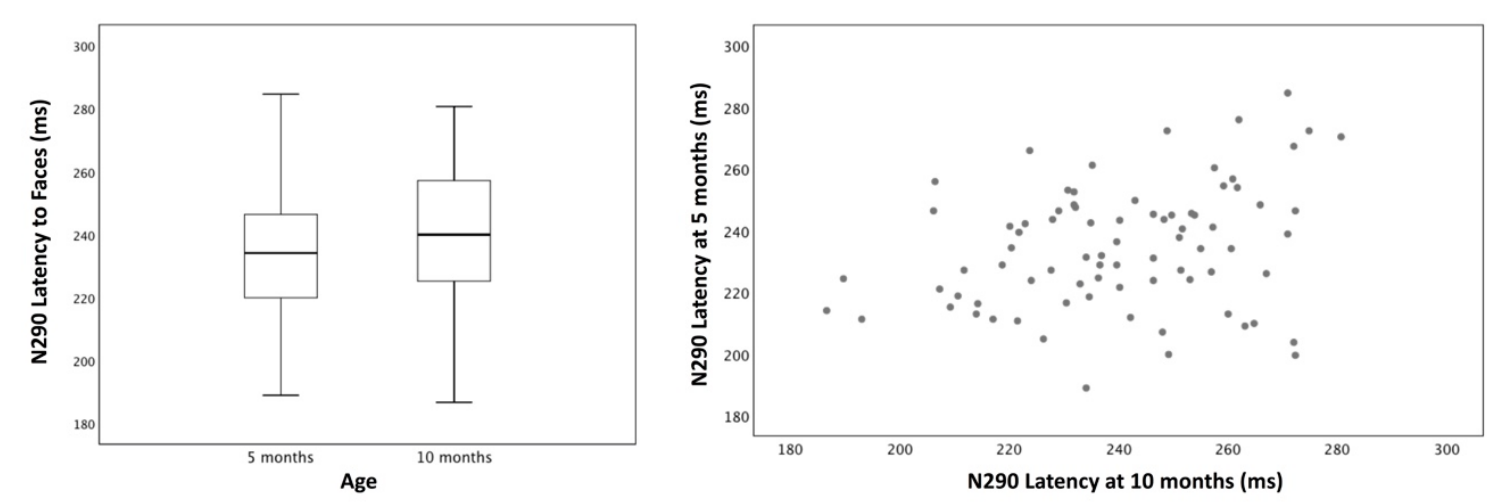

Figure S3.1 The left panel depicts the boxplots of N290 latency (in ms) recorded in response to faces at five and 10 months, horizontal line represents median while the two whiskers indicate the first and third quartile. The right panel depicts the scatterplot between N290 latency to faces at five and 10 months.

\section{Analyses on possible confounds of change between visits (or development)}

\section{Test of equal variances}

Separately for each component of interest, we tested whether the variances of the faceminus-house amplitude difference were similar between the two visits. The BrownForsythe tests shows that there is a similar variation (and therefore noise) between the face-house difference obtained at five and at 10 months of age for each component: $\mathrm{N} 290: F(1,79)=0.49, p=.49, \eta^{2}=.006 ; \mathrm{P} 400: F(1,79)=0.012, p=.91, \eta^{2}=.0001 ; \mathrm{Nc}:$ $F(1,79)=0.39, p=.53, \eta^{2}=.005$. 
Age difference at visit 1 and time interval between the two visits -We measured age at the first visit (Age visit1) and the time-interval between the two visits (Age Difference) in days (see Table S3.1). We performed the repeated measures ANOVAs reported in the main text [within subject factors: Age (5, 10 months), Stimulus type (Face, House)] with Age visit1 and Age Difference as covariates. Only for the N290 component, there is a significant interaction between Age visit1 and Stimulus type, $F(1,77)=4.89, p=.030, \eta^{2}=.060$; there are no other significant interactions with the covariates for N290 (all ps > .098), P400 (all ps > .095), and Nc (all ps > .27). We followedup the Age visit1 by Stimulus type interaction found for N290 amplitudes by computing a Pearson's correlation between Age visit1 and the mean of the face-house amplitude difference obtained at visit 1 and 2 (i.e., average of the two difference scores); this analyses revealed a weak negative correlation, $r(80)=-.28, p=.013$, which suggests a relation between age at first visit and general face-house difference. Yet, follow-up tests show no significant correlation either between Age visit1 and the face-house difference at visit $1(\mathrm{r}(80)=-.18, p=.12)$ or between Age visit1 and the face-house difference at visit $2(r(80)=-.19, p=.085)$. The lack of significant correlations at either visit suggests nonetheless that there is no relation between the variance of age and the amplitude difference at one visit. Hence, for all components, there is no reason to believe that the lack of change in face sensitivity between the two visits emerged from the ANOVA is due to these two age factors.

Table S3.1 Mean age at visit 1 and 2, and mean age difference between the two visits of infants providing EEG data for the N290/P400 and for the Nc. Age is reported in days, standard deviations are shown in the brackets.

\begin{tabular}{c|c|c|c} 
& Age visit 1 & Age visit 2 & $\begin{array}{c}\text { Age Difference } \\
\text { between visits }\end{array}$ \\
\hline N290/P400 & $168(22.5)$ & $319(25.7)$ & $151(34.2)$ \\
Nc & $168(22.0)$ & $318(25.7)$ & $150(33.5)$
\end{tabular}

\section{Number of trials}

Table S3.2 reports the number of trials per condition, per visit, and per component. Separately for each component and for each visit, we computed Pearsons' correlations 
between the face-house amplitude difference and the number of trials used to compute the house or the face mean amplitudes. Significant correlations are broken down to examine whether trial size is related to the processing of each category rather than the difference score. We do not adjust for Bonferroni corrections. If there is a relationship, we expect it to be present at both visits. Otherwise, if a relationship only holds at one visit but not at another visit, we interpret this as a Type-1 error.

\section{N290}

For visit 1 , there is a negative correlation between the face-house amplitude difference vs. the number of house trials $(r(80)=-.25, p=.022)$ as well as vs. the number of face trials $(r(80)=-.28, p=.013)$. For visit 2 , there is no significant relation between the facehouse amplitude difference and the number of house or face trials, all $p s>.28$. When we break down this correlation for visit 1 , we observe a weak positive correlation between the mean amplitude for house processing and number of trials of the house condition $(r(80)=.27, p=.015)$, whereas there is no correlation between amplitude and number of trials of the face condition $(r(80)=.015, p=.90)$. As follow-up tests show only a weak positive relation between the amplitude and the number of trials for the house condition at visit 1, while no effect emerged for the house condition at visit 2 nor for the face condition at any visits, we interpret this effect as spurious (Type-1-error).

\section{P400}

For visit 1 , we observe a negative correlation between the face-house amplitude difference vs. the number of house trials $(r(80)=-.26, p=.020)$, as well as vs. the number of face trials $(r(80)=-.31, p=.006)$. For visit 2 , there is a weak negative correlation between the face-house amplitude difference and the number of face trials $(r(80)=-.24$, $p=.032$ ), while no correlation between the amplitude difference and the house trials $(r(80)=-.071, p=.53)$. Follow-up correlations for visit 1 show a moderate positive correlation between the amplitude and number of trials of the house condition $(r(80)=$ $.41, p<.001$ ), while there is no correlation between the amplitude and number of trials of the face condition $(r(80)=.21, p=.059)$. Turning to visit 2 , there is no correlation between the mean amplitudes and number of trials of either condition, all $p s>.32$. 
Similarly to N290, the analyses on P400 show a correlation between number of trials and amplitude of the house condition at visit1, which is not surprising since these two components are recorded from the same electrode sets. Since we do not find any other significant correlations we believe that it is unlikely that the number of trials acted as a confound to the ANOVA interaction.

$\mathrm{Nc}$

There is no correlation between the face-house amplitude differences and the number of face or house trials for any visit (all $p s>.20$ ).

\section{All components}

We further computed the 2 (Age) by 2 (Stimulus type) ANOVAs only including infants with a minimum of 20 trials per variable $(n=57)$ to test if a larger number of trials (as in the original ANOVAs) masks the Stimulus by Age interaction. Results are similar. First, for each component there is a main effect of Stimulus type $(\mathrm{N} 290: F(1,56)=170$, $p<.001, \eta^{2}=.75 ; \mathrm{P} 400: F(1,56)=146, p<.0001, \eta^{2}=.72 ; \mathrm{Nc}: F(1,55)=36.5, p<.001, \eta^{2}$ $=.39)$. Second, we see a main effect of Age across components $(\mathrm{N} 290: F(1,56)=7.38, p$ $=.009, \eta^{2}=.12 ; \mathrm{P} 400: F(1,56)=21.3, p<.0001, \eta^{2}=.27 ; \mathrm{Nc}: F(1,55)=10.5, p=.002, \eta^{2}$ $=.16$ ). Third, there are no interactions between Stimulus type and Age (all $p s>.29$ ).

Together these results suggest that the difference in number of house or face trials does not mask or influence the (lack of) change observed in the main ANOVAs investigating development of face sensitivity.

Table S3.2 Mean of trials averaged for each component in response to faces and houses, at visit 1 and 2. There is no statistical difference between the number of trials included for faces and houses at each time point (all ps > .096).

\begin{tabular}{l|c|c} 
& N trials visit 1 & N trials visit 2 \\
\hline N290/P400 Face & $31.2(8.85)$ & $28.9(8.73)$ \\
N290/P400 House & $32.0(8.32)$ & $28.8(8.73)$ \\
Nc Face & $31.3(8.75)$ & $28.7(8.60)$ \\
Nc House & $32.1(8.41)$ & $28.51(8.64)$
\end{tabular}






\section{Brain responses to faces and}

\section{facial expressions in 5-month-olds: \\ an fNIRS study}

Renata Di Lorenzo

Anna Blasi

Caroline Junge

Carlijn van den Boomen

Rianne van Rooijen

Chantal Kemner

Published as:

Di Lorenzo, R., Blasi, A., Junge, C., van den Boomen, C., van Rooijen, R., \& Kemner, C. (2019). Brain responses to faces and facial expressions in 5-month-olds: an fNIRS study. Frontiers in psychology, 10, 1240. https://doi.org/10.3389/fpsyg.2019.01240

Author contribution:

$\mathrm{RDL}$ and $\mathrm{CB}$ contributed in the conception of the study. RDL and RR created the task script and collected the data. Data preprocessing was performed by RDL in collaboration with AB. RDL computed statistical analyses and drafted the manuscript. All authors contributed in the revision of the manuscript and approved the submitted version. 


\begin{abstract}
Processing faces and understanding facial expressions is a crucial skill for social communication. In adults, basic face processing and facial expression processing rely on specific interacting brain networks. In infancy, however, little is known about when and how these networks develop. The current study uses functional near-infrared spectroscopy (fNIRS) to measure differences in 5-month-olds' brain activity in response to fearful and happy facial expressions. Our results show that the right occipital region responds to faces, indicating that the face processing network is activated at five months. Yet sensitivity to emotional facial expressions appears to be still immature at this age: explorative analyses suggest that if the facial emotion processing network is active this would be mainly visible in the temporal cortex. Together these results indicate that at five months occipital areas already show face processing skills, while the facial emotion processing network seems not fully developed.
\end{abstract}




\section{Introduction}

Learning to differentiate between emotional facial expressions is vital for acquiring relevant social information. Previous research shows that infants' ability to recognize human faces and to read emotions from faces undergoes rapid changes during the first year of life. Presumably, these changes in infant behaviour are connected to changes in brain development (for reviews on how brain development links to face or facial expression processing see Haist \& Anzures, 2017, and Leppänen \& Nelson, 2009, respectively). Neuroimaging studies in adults demonstrated that the brain circuits recruited during face and facial expression processing are highly interacting (e.g., Vuilleumier \& Pourtois, 2007). Yet little is known about how these brain networks emerge in infancy. To understand which parts of the face processing and facial emotion processing networks are activated in early development, the current study measured 5month-olds' brain activity while they viewed fearful and happy facial expressions, and houses as baseline stimuli.

In adults, evidence from functional brain imaging indicates that faces evoke specific activity in the so-called core face processing network. This network constitutes three cortical areas (Haxby, Hoffman, \& Gobbini, 2002): the fusiform gyrus (FG), the occipital face area (OFA) and the superior temporal sulcus (STS). Activity in these areas has been linked to different aspects of the processing of faces. In particular, the OFA is involved in the early perception of facial features, the FG in the processing of invariant aspects of faces, and the STS is associated with detecting changeable features of faces, such as lip movements. Although facial expressions might elicit activation from parts of the 'face processing' network as well, differentiation between emotional expressions is typically observed in the 'facial emotion processing' network, which comprises the STS, the amygdala, and the orbitofrontal cortex (OFC; Leppänen \& Nelson, 2009). The amygdala rapidly responds to emotional facial expressions and successively enhances processing in cortical face-sensitive areas (i.e., FG and STS; Leppänen \& Nelson, 2009). Regarding the OFC, this region is presumably involved in emotion recognition and topdown modulation of perceptual processing (Leppänen \& Nelson, 2009). In adults, the 
connection between the networks underlying face and facial expression processing is considered to be bi-directional: the amygdala and OFC not only send information to cortical regions of the face processing network (FG and STS), but also receive information from these visual areas (Leppänen \& Nelson, 2009). While the neural basis of face and facial expression processing have been extensively studied in adults, little is known about which of these cortical areas are active in the infant brain already at five months of age.

In infancy, face categorization may possibly be innate: newborns preferentially orient towards face-like configurations rather than to non-face stimuli (Morton \& Johnson, 1991). Studies using electroencephalography (EEG) also reveal differential processing of faces as young as three months of age (earliest age reported; e.g., Halit Csibra, Volein, \& Johnson, 2004). Recently, functional near-infrared spectroscopy (fNIRS) has been used to measure changes in oxy- $(\mathrm{HbO})$ and deoxy-haemoglobin $(\mathrm{HbR})$ concentrations to infer localized cortical activity in the infant brain, which is indexed by an increase in $\mathrm{HbO}$ concentration coupled with a decrease in $\mathrm{HbR}$ concentration (for a review on fNIRS see Lloyd-Fox, Blasi, \& Elwell, 2010). Since the haemodynamic responses are restricted to cortical brain regions where neuronal activation occurs, fNIRS, like fMRI, offers a higher spatial resolution than EEG. fNIRS has been successfully used to measure infant brain responses to face stimuli, with some studies observing a right hemispheric dominance already at five months of age (Nakato et al., 2009). Face-specific activity has been revealed in the occipital and temporal areas, suggesting activation of the face processing network (Blasi et al., 2007; Honda et al., 2010; Nakato et al., 2009; Nakato et al., 2011a; Otsuka et al., 2007). However, this conclusion is based on only few studies, of which just two investigated this selectively in 4-to-5-month-olds (see research on occipital areas: Blasi et al., 2007; and occipitotemporal areas: Nakato et al., 2009). Moreover, none of these studies investigated facespecific responses using emotional faces. The conclusions thus benefit from replication and extension with such stimuli. 
Facial expression categorization also matures rapidly, albeit somewhat later than face processing. The field usually considers seven months of age the tipping point at which infants respond differently to different emotional expressions. For instance, an EEG study showed that 7-month-olds discriminated between fearful and neutral or happy facial expressions (e.g., Leppänen, Moulson, Vogel-Farley, \& Nelson, 2007; for an overview see van den Boomen, Munsters, \& Kemner, 2019). Yet two out of the four EEG studies on 4- to 5-month-olds suggest that this ability starts even earlier, by showing differential activity between happy and fearful faces (Rigato, Farroni, \& Johnson, 2009; Yrttiaho, Forssman, Kaatiala, \& Leppänen, 2014) while the other two reported no significant difference (Hoehl \& Striano, 2010; Peltola et al., 2009). No study investigated facial expression processing at this young age using fNIRS. In older infants, one NIRS study suggested that at seven months, the STS is involved in the processing of angry (right STS) and happy faces (left STS; Nakato, Otsuka, Kanazawa, Yamaguchi, \& Kakigi, 2011b). Furthermore, pictures or videos of happy faces activate the OFC in 7- to 13month-olds (Fox, Wagner, Shrock, Tager-Flusberg, \& Nelson, 2013; Minagawa-Kawai et al., 2009; Ravicz, Perdue, Westerlund, Vanderwert, \& Nelson, 2015). It remains uncertain whether temporal and frontal areas also differentially respond to different emotional expressions in infants as young as five months. As such, while some EEG studies suggest that part of the facial emotion processing network might be active at five months of age, no fNIRS study explored which parts of this network might be involved.

The current study aimed to investigate which areas of the face and the facial emotion processing networks are active during early infancy. Therefore, we used fNIRS to study these processes in three locations: the occipital (close to the OFA), the temporal (close to the STS) and the frontal (close to the OFC) cortices. As EEG studies revealed that at five months of age face processing is possible, but facial expression processing is still maturing, we studied infants at this age to capture the development of the networks at an early stage. We hypothesized that the face processing network is functioning, which would be reflected in activity at the occipital and temporal channels. This would replicate the results from previous fNIRS studies (Blasi et al., 2007; Honda et al., 2010; 
Nakato et al., 2009; Nakato et al., 2011a; Otsuka et al., 2007). Analyses on activation of the facial emotion processing network were more explorative due to the absence of fNIRS studies and the conflicting results in EEG studies. Nevertheless, we expected that if (part of) this network would be active, this would be reflected in activity in the temporal and frontal channels.

\section{Materials and methods}

\section{Participants}

We tested 17 healthy 5-month-olds (9 girls; $M_{\text {age }}=163.4$, range: $127-182$ days). Two additional infants were excluded because they viewed fewer than three trials per condition (e.g., Lloyd-Fox et al., 2013). Both parents gave written informed consent prior to participation. The Medical Ethical Committee of the University Medical Centre of Utrecht approved the study, which was conducted in accordance with the Declaration of Helsinki. Children received a book as a token for their participation.

\section{NIRS recording}

Haemodynamic responses were recorded at a $10 \mathrm{~Hz}$ sampling rate using the UCL topography half-system (NTS2; Everdell et al., 2005). Infants wore a NIRS headgear consisting of eight dual-wavelength sources $(780 \mathrm{~nm}, 850 \mathrm{~nm})$ and eight detectors, which form 22 source-detector channels at a separation of $2 \mathrm{~cm}$ (Figure 4.1A). The probe array covered parts of the right occipital, temporal and frontal cortices (Lloyd-Fox et al., 2014).

To standardize the headgear position across participants, external scalp landmarks were used as reference (Blasi et al., 2014). The headgear was placed over the right hemisphere with detector 5 centered above the pre-auricular point (T4 according to the 10-20 system; vertical axis in Figure 4.1B). The lower edge of the probe band was aligned to the line between the top of the ear lobe and the highest point of the eyebrow (horizontal axis in Figure 4.1B). The headgear position was checked before and after the experiment; photos were taken to review (shifts of) band placement. No child was 
excluded for incorrect NIRS sensor placement, defined as a shift of more than $1 \mathrm{~cm}$ on the horizontal or vertical axes from the reference point.
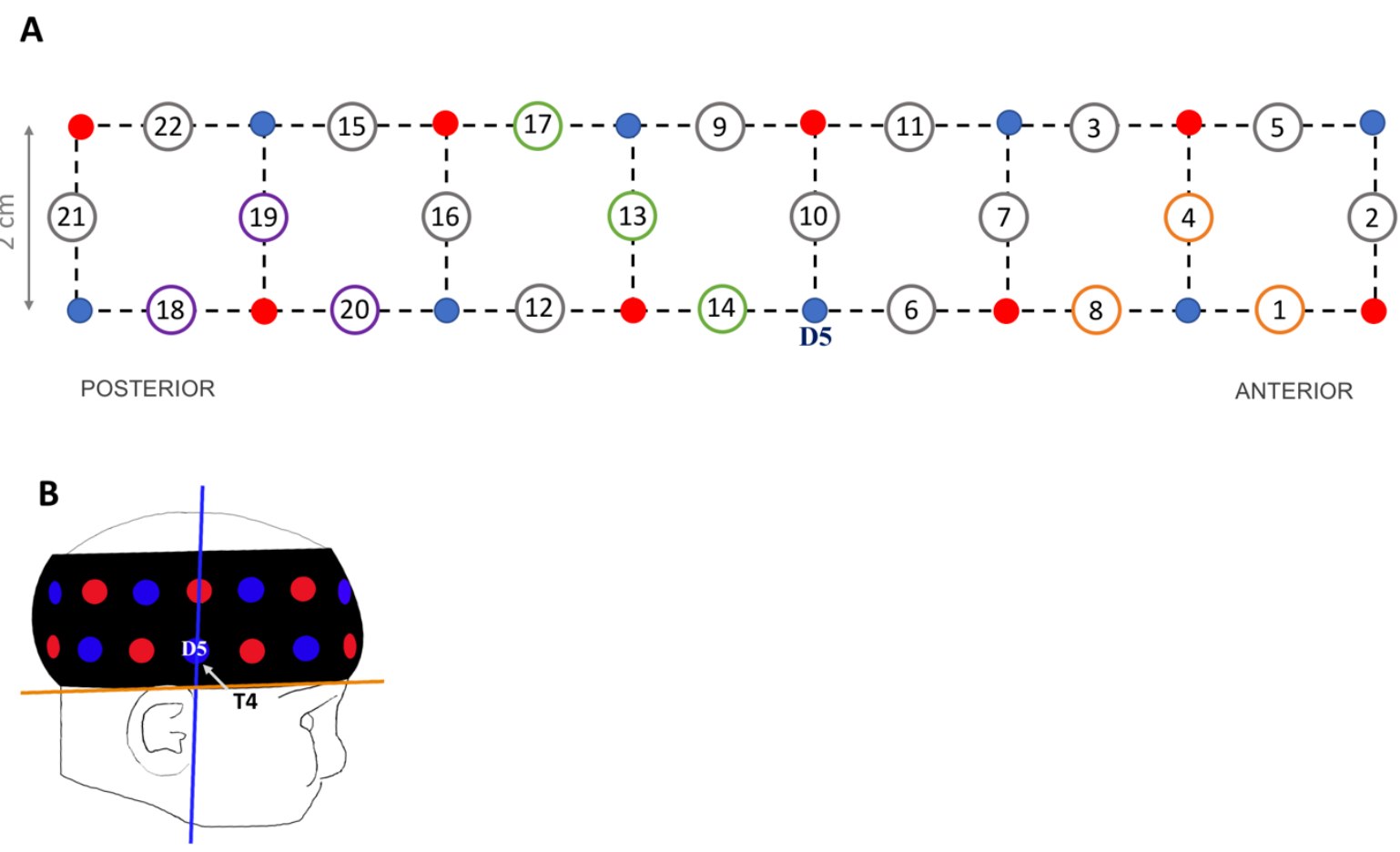

Figure 4.1 (A) Headgear design showing the position of sources (red dots), detectors (blue dots) and channels at a 2-cm source-detector separation (circled numbers). Channels of interests are 3 occipital (purple circles), 3 temporal (green circles) and 3 frontal (orange circles). (B) Illustration of a 5-monthold wearing the probe band covering the right hemisphere, with the blue line corresponding to optimal vertical position of detector 5 (D5, T4 according to the 10-20 system) and orange line to optimal horizontal position.

\section{Stimuli}

We selected six female models expressing fear or happiness from the Radboud Faces Database (see Figure 4.2; identities: 12, 22, 26, 27, 37, 61; Langner et al., 2010). Baseline stimuli were 12 pictures of houses selected from the internet. The coloured images were depicted on a grey background (RGB: 108) and measured $20.5 \mathrm{~cm}$ width x $22.5 \mathrm{~cm}$ height (visual angle: $19.4^{\circ} \times 21.2^{\circ}$ ).

\section{Procedure}

During the study infants sat on their parent's lap, at $60 \mathrm{~cm}$ from a 23 -inch computer monitor (refresh rate $60 \mathrm{~Hz}, 1920$ x 1080 resolution), in a semi-dark room. Parents were 
instructed not to interact with their child during the experiment. A video camera placed on top of the screen recorded the child's behaviour. The task was programmed in Matlab using Psych-Toolbox 3 (Brainard \& Vision, 1997). It comprised alternating 5-second experimental trials displaying sequences of five either happy or fearful expressions interleaved with baseline trials, of minimum $10 \mathrm{~s}$, displaying sequences of at least 10 houses ( $M=12.2$ houses; $S D=.69$; range $=10-22$; Figure 4.2 depicts the task design). Every stimulus was presented for $800 \mathrm{~ms}$, followed by a $200 \mathrm{~ms}$ interstimulus interval presenting a fixation cross; the order of the stimuli was randomized within trials. While the task always started with a baseline trial, the order of experimental conditions (fearful and happy facial expressions) was counterbalanced across infants. The ending of a baseline trial was controlled by the experimenter, who continued to the next trial only when the infant was attending the screen. To make the task more interesting, non-social sounds were randomly played after every 1-2 pictures, which caused a short delay in the following stimulus onset, producing the jittering of stimulus presentation. Only during baseline trials, the experimenter could play additional sounds to redirect the child's attention to the screen. The experiment lasted on average 5 minutes and ended after all 10 trials per condition were presented or when an infant became too restless.

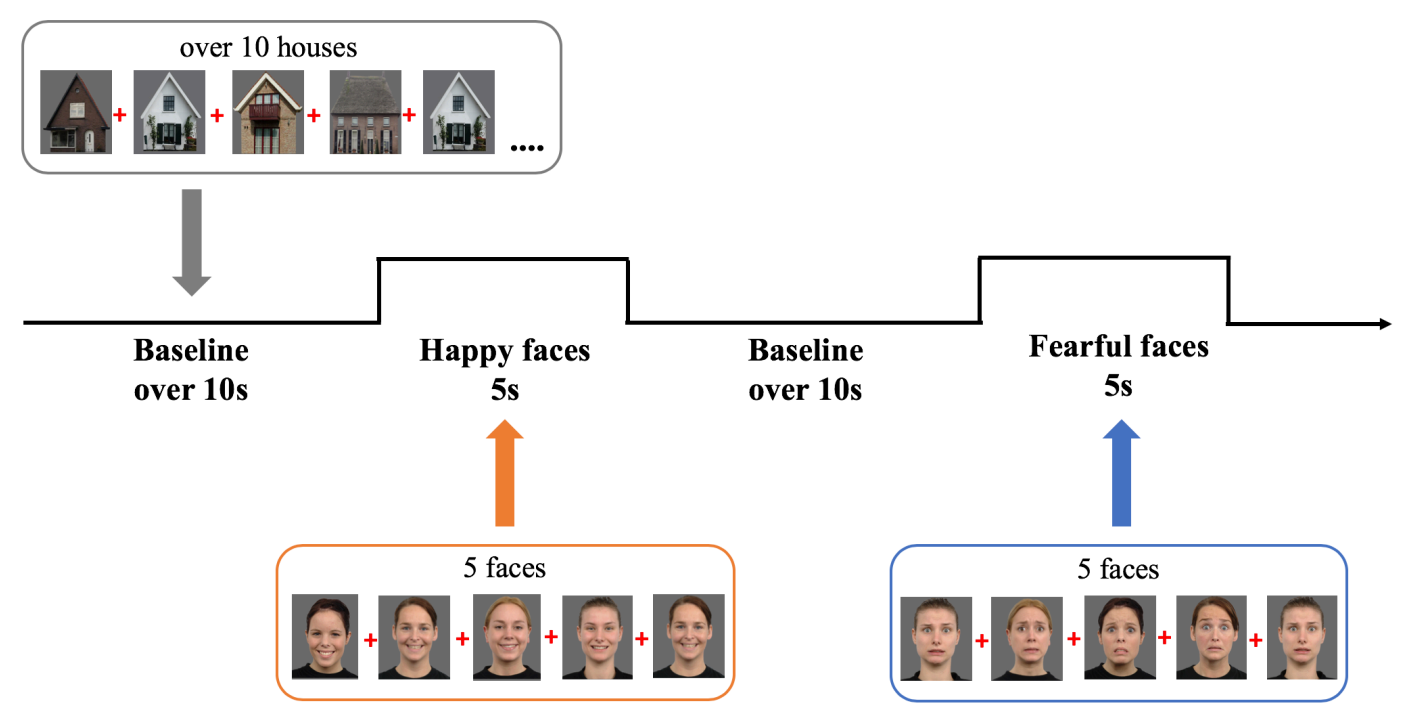

Figure 4.2 Experimental design showing the order and timing of stimulus presentation for the experimental (five pictures of female faces expressing either happiness or fear) and baseline trials (at least $10 \mathrm{~s}$ of pictures of houses). 


\section{Data processing}

First, we coded infants' looking behaviour offline and scored their compliance with the study. We excluded trials when the infant looked at the screen for less than $60 \%$ of the trial duration. Infants completed an average of 7 trials per condition $(S D=2.40$; fearful condition: range 3-10; happy condition: range 3-9 trials).

The NIRS data were then processed with Homer2 (MGH-Martinos Center for Biomedical Imaging, Boston, USA). First, channels with very low or high intensity levels were discarded with the enPrunechannel function. Raw intensity data of the remaining channels were converted to optical density units. Spline interpolation $(p=0.99)$ and wavelet $(\mathrm{iqr}=0.80)$ functions in Homer2 were used to correct for motion artifacts in the data. Motion artifacts were detected by the function hmrMotionArtifactByChannel $(\mathrm{AMP}=0.40, \mathrm{SDThresh}=15.5$, tMotion $=1.0$, tMask $=1.0)$. We excluded trials with artifacts in the $[-2+5 \mathrm{~s}]$ time window that survived the correction. The data was then band-pass filtered $(0.030-0.80 \mathrm{~Hz})$ and changes in concentration of $\mathrm{HbO}$ and $\mathrm{HbR}$ were calculated using the modified Beer-Lambert Law, with a pathlength factor of 5.1 (Duncan et al., 1995). Finally, we computed a block average for $2 \mathrm{~s}$ pre and $15 \mathrm{~s}$ poststimulus onset; the time window $[-2-0 \mathrm{~s}]$ was used for baseline correction.

We selected three channels to encompass each region of interest, based on a NIRSMRI co-registration scalp anatomical map of 4- to 6-month-olds (Lloyd-Fox et al., 2014) and on the 10-20 system coordinates typically used in EEG. For the occipital region we selected channels 18, 19 and 20 located approximately between T6 and O2 (channels nearest to the OFA region); for the temporal region channels 13,14 , and 17 (surrounding the STS region); and for the frontal region channels 1,4 , and 8 (corresponding approximately to the OFC region). For each infant, the haemodynamic responses of every ROI were calculated by averaging the responses across the channels within each region, separately for each condition. 


\section{Statistical Analyses}

Maximum haemodynamic changes (peak amplitudes) were calculated for both $\mathrm{HbO}$ and $\mathrm{HbR}$. Note that typically, in infant research, $\mathrm{HbO}$ is the preferred measure of activation, as it has higher SNR and is more consistent compared to HbR concentration change in infant studies (for a more in depth discussion see Lloyd-Fox et al., 2010). Therefore, we will focus the discussion on the $\mathrm{HbO}$ results. To investigate differences in the timing of the response across experimental conditions (Nakato et al., 2011b) we selected two relatively narrow time windows for statistical analyses: 3-8 and 8-13 s poststimulus onset. These periods of time were chosen to include the range of maximum concentration changes observed across infants for $\mathrm{HbO}$ and $\mathrm{HbR}$, based on visual inspection of the grand-averaged haemodynamic responses and in accordance with previous work published with a similar task (e.g., Lloyd-Fox et al., 2013, 2017). Previous research that tested a similar age group with face stimuli showed that the haemodynamic response is expected to peak after the end of the experimental trial (e.g., Lloyd-Fox et al., 2017).

Statistical tests were performed using IBM SPSS Statistics 25.0 (IBM Corporation, Armonk, NY, USA). We carried out separate 3-way repeated measures ANOVAs to investigate changes in $\mathrm{HbO}$ or $\mathrm{HbR}$ in response to the different Emotional expressions (Fearful and Happy) across the regions of interest (Temporal, Frontal and Occipital) and across the two Time Windows. Overall, this analysis captures both effects of face processing and facial expression processing. In particular, face processing will be indexed by main effects of ROI or Time Window, which correspond to differences in the activity evoked by face stimuli between regions or timing, respectively. At the same time, expression discrimination would be reflected by any main effect or interaction with emotional expression resulting from the ANOVA. Significant effects will be followed up with paired and one-sample t-tests. Since no prior fNIRS study tested facial expression discrimination in 5-month-olds, a set of exploratory analyses will be run to look at effects of happy vs. fearful expressions at each of the three ROI, for both $\mathrm{HbO}$ 
and $\mathrm{HbR}$ values, even in the case of an absence of significant interaction of Emotion and ROI.

We report additional one-sample and paired t-tests (channel by channel, for $\mathrm{HbO}$ and HbR) in the Supplementary Materials III, as these analyses are more commonly reported in infant fNIRS studies. Overall, uncorrected analyses revealed significant effects of fearful and happy faces on $\mathrm{HbO}$ in the occipital and temporal, but not frontal regions. However, these results did not survive FDR correction for 22 comparisons.

\section{Results}

Figure 4.3 and 4.4 depict the haemodynamic response to happy and fearful faces. The ANOVA on $\mathrm{HbO}$ activity yielded a significant main effect of ROI $(F(2,32)=3.67, p=$ $\left..037, \eta^{2}=.19\right)$. Follow-up paired sample t-tests revealed that $\mathrm{HbO}$ was significantly higher at Occipital $(M=.24, S D=.36)$ than Temporal regions $(M=-.059, S D=.24 ; t(16)$ $=2.9, p=.010, d=.70)$, while no difference was found between Frontal $(M=.051, S D=$ $.32)$ vs. Occipital $(t(16)=-1.52, p=.15, d=.39)$, or Frontal vs. Temporal regions $(t(16)$ $=1.03, p=.32, d=.25)$. Yet, the one sample t-tests assessing whether the face response deviated significantly from zero indicated that only for the Occipital region face stimuli elicited a significant increase in $\mathrm{HbO}(t(16)=2.8, p=.012, d=.68)$; no other difference was found significant (Temporal: $t(16)=-1.01, p=.33, d=.24$; Frontal: $t(16)=0.66, p$ $=.52, d=.16)$.

There was a marginal effect of Emotion $\left(F(1,16)=4.06, p=.061, \eta^{2}=.20\right)$ reflecting a trend towards higher $\mathrm{HbO}$ values for Fearful $(M=.19, S D=.26)$ than Happy expressions $(M=-.028, S D=.28)$, and a marginal interaction between Emotion and ROI $\left(F(2,32)=3.17, p=.055, \eta^{2}=.17\right)$. No other significant effects were found. The ANOVA on $\mathrm{HbR}$ values yielded no significant effects $(\min F=.065 ; \min p=.097)$.

Based on the explorative nature of the investigation of facial expression processing at this age and the marginal effect of Emotion and marginal interaction between Emotion and ROI, we further looked into emotion responses in each region to help guide future studies. The paired t-test performed on $\mathrm{HbO}$ values revealed that only for 
the Temporal region $\mathrm{HbO}$ was significantly higher for Fearful $(M=.14, S D=.39)$ than Happy faces $(M=-.26 ; S D=.45), t(16)=2.38, p=.030, d=.59$; no difference between facial expressions was found for the Occipital (Fearful: $M=.31, S D=.43$; Happy: $M=$ .18, $S D=.39 ; t(16)=1.34, p=.20, d=.32$ ), nor for the Frontal areas (Fearful: $M=.11$, $S D=.33$; Happy: $M=-.005, S D=.45 ; t(16)=0.99, p=.34, d=.25)$. Follow-up onesample t-tests performed for the Temporal $\mathrm{HbO}$ values indicated that the effect of Emotion was due to $\mathrm{HbO}$ non-significantly increasing for Fearful faces $(t(16)=1.51, p$ $=.15, d=.38)$ while significantly decreasing for Happy faces $(t(16)=-2.36, p=.031, d=$ -.59). The exploratory paired t-tests examining emotion differences computed on $\mathrm{HbR}$ values yielded no significant difference between facial expressions in any ROI ( $\min t=$ $.92, \min p=.22)$.

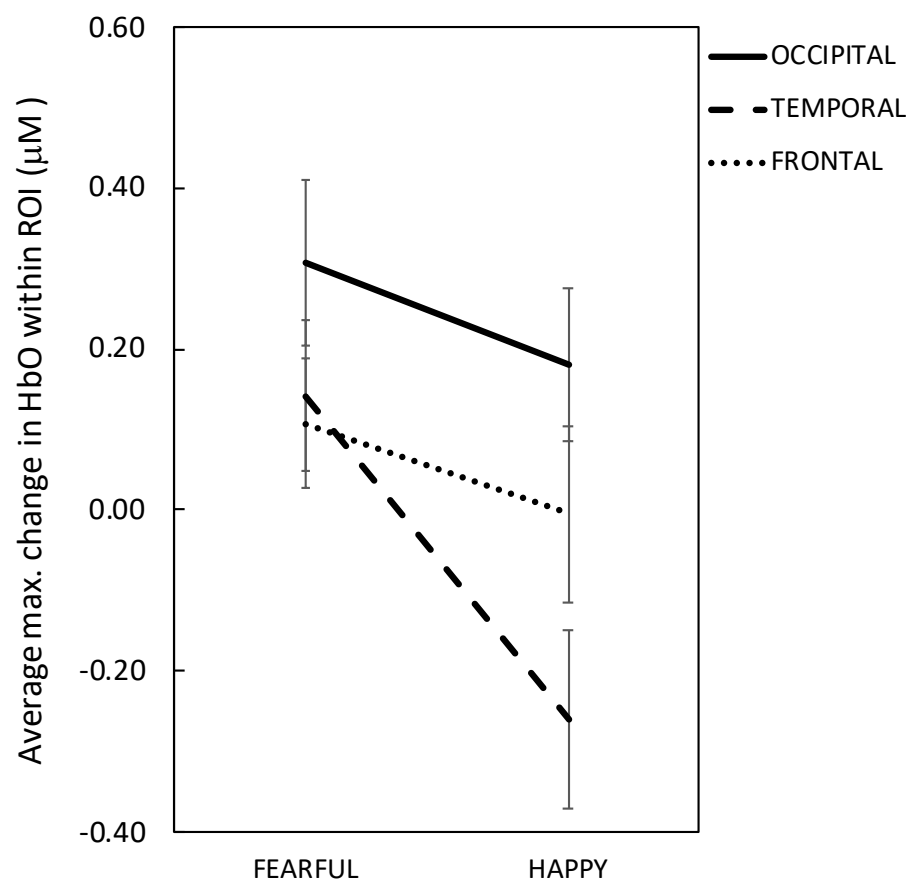

Figure 4.3 Maximum change (or peak amplitude) in $\mathrm{HbO}$ averaged across channels within each ROI per Emotion condition (Fearful, Happy). Solid line denotes occipital ROI, dashed line denotes temporal ROI and dotted line denotes frontal ROI. Error bars represent the standard error. 


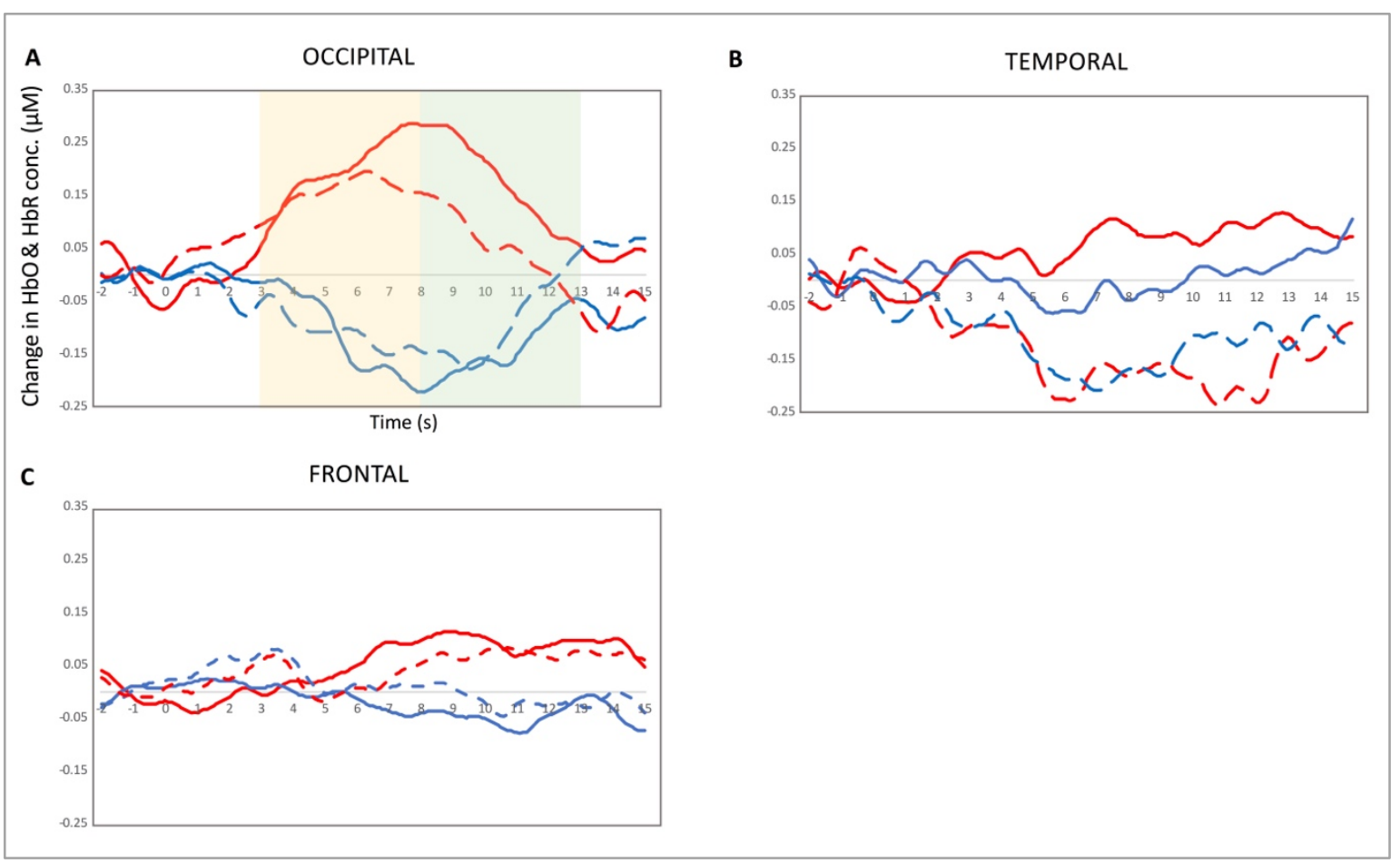

Figure 4.4 Grand averaged haemodynamic responses (in $\mu \mathrm{M}$ ) to happy (dashed line) and fearful (solid line) faces, recorded from occipital (A), temporal (B) and frontal (C) regions. $\mathrm{HbO}$ is in red and $\mathrm{HbR}$ is in blue. The yellow and green areas in the upper left panel highlight the early and late time windows, respectively; $\mathrm{t}=0$ indicates stimulus onset.

\section{Discussion}

This research aimed to investigate which cortical areas are sensitive to basic face processing and explore which ones are involved in facial expression processing in 5month-old infants. Overall, our results indicate that the right occipital area responds to faces regardless of emotional expression. A marginal emotion by region interaction seemed to relate to differences between facial expressions in the temporal region. Surprisingly, explorative analyses revealed that happy facial expressions elicit a significant decrease in $\mathrm{HbO}$ at temporal sites, while $\mathrm{HbO}$ concentrations followed the opposite direction in the fearful condition (albeit not significantly above zero). Generally, these results suggest that the face processing network is active in 5-montholds, while activity in the facial emotion processing network appears not yet mature.

Our findings on the involvement of occipital areas in general face processing align with previous infant fNIRS research: a significant increase in $\mathrm{HbO}$ in response to faces. 
For instance, one study with 4-month-olds reports that compared to visual noise, neutral faces reveal more widespread $\mathrm{HbO}$ increases in occipital channels (Blasi et al., 2007). Similarly, another study with 5-month-olds observes that neutral faces elicit $\mathrm{HbO}$ increases in channels placed between T6 and O2 (Nakato et al., 2009), although note that the authors attribute this activation to the nearby region of STS. The current study adds to these results that, at five months of age, the occipital cortex is also sensitive to faces with different emotional expressions, while the temporal and frontal regions do not show such sensitivity (note that the activity recorded at the occipital region did not differ from that at the frontal region as the latter showed a small but non-significant $\mathrm{HbO}$ increase). This occipital region is also close to a cortical area involved in face processing in the adult brain: the OFA. This region appears to be involved in the early perception of facial features; previous adult fMRI studies report an increased activity for faces versus other objects (Haxby et al., 2002). Therefore, we interpret this occipital activation as reflecting OFA response to facial features common to both facial expressions. In the temporal and frontal regions we did not observe such increases of $\mathrm{HbO}$ irrespective of facial expression. Thus, our study provides further support that infants at this age can recruit occipital regions for basic face processing.

Next, we turn to our exploration of whether different facial expressions elicit different patterns of cortical activity in 5-month-olds. Our results suggest that only temporal sites show a possible sensitivity to different facial expressions, while the frontal regions did not. Based on the marginal results, no strong conclusion can be drawn. Possibly, the infant brain is not yet sensitive to fearful and happy facial expressions at five months of age. This suggestion is consistent with findings of some EEG studies (Hoehl \& Striano, 2010; Peltola et al., 2009) and with the Leppänen and Nelson model (2009) on maturation of facial emotion processing, confirming that the network is still immature at five months of age. Possibly the infant brain requires additional exposure to different types of facial expressions before the relevant brain areas become engaged with processing emotions within faces. Another possibility is that the facial emotion processing network already shows some sensitivity to facial expressions. This option in 
is line with other EEG studies (Rigato et al., 2010; Yrttiaho et al., 2014). The current results reveal that if this is the case, the temporal region is the first part of the network to become active. Although the temporal region is where one would expect to differentiate between facial expressions, our results do not show the predicted pattern. We observe that there was a strong $\mathrm{HbO}$ decrease for happy expressions, coupled with a non-significant $\mathrm{HbO}$ increase for fearful expressions.

What can a decrease in $\mathrm{HbO}$ reflect? The general consensus in the fNIRS community is that an $\mathrm{HbO}$ decrease cannot be interpreted as cortical activation; only an increase of $\mathrm{HbO}$ and/or a decrease in $\mathrm{HbR}$ are considered markers of brain activity, with increases of $\mathrm{HbO}$ being the most favored measure of activation in infant research (for discussion see Lloyd-Fox et al., 2010). Nevertheless, there have been a number of infant studies that occasionally report significant decreases in HbO. Ravicz and colleagues (2015) reported such a decrease evoked by happy faces in frontal channels in 7-montholds as well. They suggest there may be multiple potential causes for an $\mathrm{HbO}$ decrease: (i) it could be related to an unsteady and immature neurovascular coupling in infancy (Kozberg et al., 2013); (ii) it could also indicate neural deactivation, with the blood supply from that region being diverted to nearby areas that require oxygen (Wilcox et al., 2009); (iii) or it might well be a deactivation compared to baseline. However, these suggestions cannot fully explain our pattern of results: for instance, it is unlikely that neurovascular coupling is immature for happy but not the fearful faces, or that activation in the occipital region leads to a deflux of oxygen in the temporal region for happy faces only. Clearly, it remains puzzling why infant studies sometimes encounter decreases in $\mathrm{HbO}$ for one condition but not for another. More research is required to elucidate when and why this decrease in $\mathrm{HbO}$ appears before we can fully interpret the haemodynamic pattern observed in the temporal region.

It is also possible that with a larger sample size our weak findings of differential activation for the temporal region become clearer towards either direction of significance. It is important to note that even though we have a small sample size, our study matches the sample sizes typically employed in infant fNIRS studies (for an 
overview see Lloyd-Fox et al., 2010). With small sample sizes even small individual differences could mask group effects. Also, it could be that this individual variation is meaningful, and can be traced back to differences in brain maturation, every-day experience, or personality (e.g., Ravicz, 2015). More research is needed to understand the scope of individual variation in fNIRS infant studies. One further limitation of this study is that although we extensively researched the involvement of different regions in the right hemisphere, we cannot draw any conclusions on the involvement of the left hemisphere. The reason why we focus on the right hemisphere is because several studies suggest that this hemisphere is dominant for face processing even in infancy (e.g., Nakato et al., 2009; Otsuka et al., 2007). However, one infant study with 7-month-olds reveals that the left STS responds more to happy than to angry facial expressions (Nakato et al., 2011b). More research is required to fully understand the contribution of the left temporal region for facial expression discrimination.

In summary, the present study provides further evidence that at five months of age infants recruit occipital areas while viewing faces, but also highlights that their cortical emotion-encoding mechanisms are still immature. Further work is needed to disentangle when and how across development the infant brain becomes able to discriminate between facial expressions.

\section{Acknowledgements}

We thank the parents and infants for their involvement in this study and Sabrina Brigadoi and Laura Pirazzoli for helping with the NIRS data preprocessing. 


\section{References}

Blasi, A., Fox, S., Everdell, N., Volein, A., Tucker, L., Csibra, G., ... \& Elwell, C. (2007). Investigation of depth dependent changes in cerebral haemodynamics during face perception in infants. Physics in medicine and biology, 52(23), 6849. http://doi.org/10.1088/0031-9155/52/23/005

Blasi, A., Lloyd-Fox, S., Johnson, M., \& Elwell, C. (2014). Test-retest reliability of functional near infrared spectroscopy in infants. Neurophotonics, 1(2). http://doi.org/10.1117/1.NPh.1.2.025005

Brainard, D., \& Vision, S. (1997). The psychophysics toolbox. Spatial vision, 10, 433-436.

Duncan, A., Meek, J., Clemence, M., Elwell, C., Tyszczuk, L., Cope, M., \& Delpy, D. (1995). Optical pathlength measurements on adult head, calf and forearm and the head of the newborn infant using phase resolved optical spectroscopy. Physics in Medicine and Biology, 40(2), 295-304. http://doi.org/10.1088/0031-9155/40/2/007

Everdell, N., Gibson, A., Tullis, I., Vaithianathan, T., Hebden, J., \& Delpy, D. (2005). A frequency multiplexed near-infrared topography system for imaging functional activation in the brain. Review of Scientific Instruments, 76(9), 1-6. http://doi.org/10.1063/1.2038567

Fox, S., Wagner, J., Shrock, C., Tager-Flusberg, H., \& Nelson, C. (2013). Neural processing of facial identity and emotion in infants at high-risk for autism spectrum disorders. Frontiers in human neuroscience, 7. http://doi.org/10.3389/fnhum.2013.00089

Haist, F., \& Anzures, G. (2017). Functional development of the brain's face-processing system. Wiley Interdisciplinary Reviews: Cognitive Science, 8(1-2), 1-12.

Halit, H., Csibra, G., Volein, A., \& Johnson, M. H. (2004). Face-sensitive cortical processing in early infancy. Journal of Child Psychology and Psychiatry, 45(7), 1228-1234. http://doi.org/10.1111/j.1469-7610.2004.00321.x

Haxby, J., Hoffman, E., \& Gobbini, M. (2002). Human neural systems for face recognition and social communication. Biological psychiatry, 51(1), 59-67.

Hoehl, S., Striano, T., 2010b. The development of emotional face and eye gaze processing. Dev. Sci. 13 (6), 813-825. http://doi.org/10.1111/j.1467-7687.2009.00944.x.

Honda, Y., Nakato, E., Otsuka, Y., Kanazawa, S., Kojima, S., Yamaguchi, M., \& Kakigi, R. (2010). How do infants perceive scrambled face?: A near-infrared spectroscopic study. Brain research, 1308, 137-146. http://doi.org/10.1016/j.brainres.2009.10.046

Kozberg, M. G., Chen, B. R., DeLeo, S. E., Bouchard, M. B., \& Hillman, E. M. (2013). Resolving the transition from negative to positive blood oxygen level-dependent responses in the developing brain. Proceedings of the National Academy of Sciences, 201212785. 
Langner, O., Dotsch, R., Bijlstra, G., Wigboldus, D., Hawk, S., \& van Knippenberg, A. (2010). Presentation and validation of the Radboud Faces Database. Cognition \& Emotion, 24(8), 1377-1388. http://doi.org/10.1080/02699930903485076

Leppänen, J., \& Nelson, C. (2009). Tuning the developing brain to social signals of emotions. Nature Reviews Neuroscience, 10(1), 37-47. http://doi.org/10.1038/nrn2554

Leppänen, J., Moulson, M., Vogel-Farley, V., \& Nelson, C. (2007). An ERP study of emotional face processing in the adult and infant brain. Child development, 78(1), 232-245. http://doi.org/10.1111/j.1467-8624.2007.00994.x

Lloyd-Fox, S., Blasi, A., \& Elwell, C. (2010). Illuminating the developing brain: The past, present and future of functional near infrared spectroscopy. Neuroscience and Biobehavioral Reviews, 34(3), 269-284. http://doi.org/10.1016/j.neubiorev.2009.07.008

Lloyd-Fox, S., Blasi, A., Elwell, C., Charman, T., Murphy, D., \& Johnson, M. (2013). Reduced neural sensitivity to social stimuli in infants at risk for autism. Proceedings of the Royal Society B: Biological Sciences, 280(1758), 20123026-20123026.

http://doi.org/10.1098/rspb.2012.3026

Lloyd-Fox, S., Richards, J., Blasi, A., Murphy, D., Elwell, C., \& Johnson, M. (2014). Coregistering functional near-infrared spectroscopy with underlying cortical areas in infants. Neurophotonics, 1(2), 025006-025006. http://doi.org/10.1117/1.NPh.1.2.025006

Lloyd-Fox, S., Begus, K., Halliday, D., Pirazzoli, L., Blasi, A., Papademetriou, M., ... \& Elwell, C. E. (2017). Cortical specialisation to social stimuli from the first days to the second year of life: A rural Gambian cohort. Developmental cognitive neuroscience, 25, 92-104.

Minagawa-Kawai, Y., Matsuoka, S., Dan, I., Naoi, N., Nakamura, K., \& Kojima, S. (2009). Prefrontal activation associated with social attachment: facial-emotion recognition in mothers and infants. Cerebral Cortex, 19(2), 284-292.

http://doi.org/10.1093/cercor/bhn081

Morton, J., \& Johnson, M. (1991). CONSPEC and CONLERN: a two-process theory of infant face recognition. Psychological review, 98(2), 164. http://doi.org/10.1037/0033295X.98.2.164

Nakato, E., Otsuka, Y., Kanazawa, S., Yamaguchi, M. K., Honda, Y., \& Kakigi, R. (2011a). I know this face: Neural activity during mother'face perception in 7-to 8-month-old infants as investigated by near-infrared spectroscopy. Early human development, $87(1)$, $1-7$.

Nakato, E., Otsuka, Y., Kanazawa, S., Yamaguchi, M., \& Kakigi, R. (2011b). Distinct differences in the pattern of hemodynamic response to happy and angry facial expressions in infants-A near-infrared spectroscopic study. NeuroImage, 54(2), 16001606. http://doi.org/10.1016/j.neuroimage.2010.09.021 
Nakato, E., Otsuka, Y., Kanazawa, S., Yamaguchi, M., Watanabe, S., \& Kakigi, R. (2009). When do infants differentiate profile face from frontal face? A near-infrared spectroscopic study. Human Brain Mapping, 30(2), 462-472. http://doi.org/10.1002/hbm.20516

Obrig, H., \& Villringer, A. (2003). Beyond the visible-imaging the human brain with light. Journal of Cerebral Blood Flow \& Metabolism, 23(1), 1-18. http://doi.org/10.1097/01.WCB.0000043472.45775.29

Otsuka, Y., Nakato, E., Kanazawa, S., Yamaguchi, M., Watanabe, S., \& Kakigi, R. (2007). Neural activation to upright and inverted faces in infants measured by near infrared spectroscopy. NeuroImage, 34(1), 399-406.

http://doi.org/10.1016/j.neuroimage.2006.08.013

Peltola, M.J., Leppänen, J.M., Mäki, S., Hietanen, J.K., 2009. Emergence of enhanced attention to fearful faces between 5 and 7 months of age. Social Cognitive Affective Neuroscience. 4 (2), 134-142. http://dx.doi.org/10.1093/scan/nsn046.

Ravicz, M. M., Perdue, K. L., Westerlund, A., Vanderwert, R. E., \& Nelson, C. A. (2015). Infants' neural responses to facial emotion in the prefrontal cortex are correlated with temperament: a functional near-infrared spectroscopy study. Frontiers in psychology, 6, 922.

Rigato, S., Farroni, T., \& Johnson, M. (2009). The shared signal hypothesis and neural responses to expressions and gaze in infants and adults. Social Cognitive and Affective Neuroscience, 5(1), 88-97. http://doi.org/10.1093/scan/nsp037

van den Boomen, C., Munsters, N., \& Kemner, C. (2019). Emotion processing in the infant brain: The importance of local information. Neuropsychologia, 126, 62-68. https://doi.org/10.1016/j.neuropsychologia.2017.09.006

Vuilleumier, P., \& Pourtois, G. (2007). Distributed and interactive brain mechanisms during emotion face perception: Evidence from functional neuroimaging. Neuropsychologia, 45(1), 174-194. http://doi.org/10.1016/j.neuropsychologia.2006.06.003

Wilcox, T., Bortfeld, H., Woods, R., Wruck, E., Armstrong, J., \& Boas, D. (2009). Hemodynamic changes in the infant cortex during the processing of featural and spatiotemporal information. Neuropsychologia, 47(3), 657-662. http://doi.org/10.1016/j.neuropsychologia.2008.11.014

Yrttiaho, S., Forssman, L., Kaatiala, J., Leppänen, J.M., 2014. Developmental precursors of social brain networks: the emergence of attentional and cortical sensitivity to facial expressions in 5 to 7 months old infants. PloS One 9 (6), e100811. http://doi.org/10.1371/journal.pone.0100811. 


\section{Supplementary Materials III}

We also analyzed our data channel-by-channel, by calculating one-sample (Fearful faces vs. zero; Happy faces vs. zero) and paired t-tests (fearful vs. happy). Table S4.1 summarizes the significant haemodynamic responses at $\mathrm{p}<.05$ ( $\mathrm{HbO}$ and $\mathrm{HbR}$ ) to happy and fearful facial expressions for both time windows (i.e., early: 3-8s, late: 8-13s).

One-sample t-tests: When comparing the pattern of significant differences for each emotion type, we observe that for both the early and the late time windows there are more occipital and temporal channels showing a significant increase in $\mathrm{HbO}$ to fearful faces (early: three; late: three) as compared to happy faces (only one with an increase in the early time window for an occipital channel). Surprisingly, the happy condition elicited a significant $\mathrm{HbO}$ decrease from three temporal channels $(9,11,17)$ during the early time window and from one channel (17) in the late time window. Further, there is one frontal channel (3) showing a significant decrease in HbR for fearful faces.

Paired-Sample t-tests: Results of the paired t-tests reveal that fearful facial expressions evoke greater responses compared to happy expressions in a temporal channel during the late time window: $\mathrm{HbO}$ channel 10: $t(16)=0.73, \mathrm{p}=.01$.

However, none of the results presented here survive the FDR correction.

Table S4.1 Results from the one-sample t-test, channel-by-channel analysis. Significant increases or decreases of $\mathrm{HbO}$ and $\mathrm{HbR}$ concentrations are reported for both conditions $(p<.05)$. TW indicates the time windows selected in this study.

\begin{tabular}{cccccccc} 
& Channel & Measure & Condition & Region & p-value & tstat & df \\
\hline TW 3-8s & & & & & & & \\
\hline & 14 & HbO & Fearful & Temporal & .035 & 2.30 & 16 \\
& 18 & HbO & Fearful & Occipital & .030 & 2.39 & 16 \\
& 19 & HbO & Fearful & Occipital & .027 & 2.43 & 16 \\
& 9 & HbO & Happy & Temporal & .030 & -2.38 & 16 \\
& 11 & HbO & Happy & Fronto-Temporal & .007 & -3.12 & 16 \\
& 17 & HbO & Happy & Temporal & .045 & -2.17 & 16 \\
TW 8-13s & 18 & HbO & Happy & Occipital & .033 & 2.33 & 16 \\
& & & & & & & \\
& 10 & HbR & Fearful & Frontal & .014 & -2.76 & 16 \\
& 12 & HbO & Fearful & Temporal & .016 & 2.70 & 16 \\
& 18 & HbO & Fearful & Temporal & .007 & 3.13 & 15 \\
& 17 & HbO & Happy & Temporal & .031 & -2.36 & 16
\end{tabular}




Is it fear?

Similar brain responses to fearful and neutral faces

\title{
in infants with a heightened likelihood for Autism Spectrum Disorder
}

\author{
Renata Di Lorenzo \\ Nicolette Munsters \\ Emma K. Ward \\ Maretha de Jonge \\ Chantal Kemner \\ Carlijn van den Boomen
}

Under revision for publication

Author contribution:

$\mathrm{CB}, \mathrm{MJ}$ and $\mathrm{CK}$ contributed to the study conceptualization. $\mathrm{CB}$ and NM ideated the task design. RDL, NM, and EW contributed to data collection. RDL was responsible for data preprocessing and statistical analyses. RDL wrote the first draft of the manuscript and formulated the specific hypotheses; $\mathrm{CB}$ and $\mathrm{CK}$ provided critical feedback on several drafts of this manuscript; NM contributed in finalizing the manuscript. All authors provided feedbacks and approved the final manuscript. 


\begin{abstract}
Individuals with autism spectrum disorder (ASD) show atypical processing of facial expressions. Research with autistic toddlers suggests that abnormalities in processing of spatial frequencies (SF) contribute to such differences. The current event-relatedpotential (ERP) study investigated differences between 10-month-old infants with highand low-likelihood for ASD in the processing of SF and of facial expressions (i.e. fearful and neutral) filtered to contain specific SF. Results indicate no group differences in general processing of higher- (HSF, detailed) and lower-SF (LSF, global) information. However, unlike low-likelihood infants, high-likelihood infants do not show differential brain responses to facial expressions when either the LSF or HSF information is available. Combined with previous findings in toddlers, the current results indicate a developmental delay in efficient processing of facial expressions in ASD.
\end{abstract}




\section{Introduction}

Difficulties in social communication are a prominent and defining characteristic of autism spectrum disorder (ASD; American Psychiatric Association, 2013). It has been previously hypothesized that these behavioral difficulties in individuals with ASD relate to differences in the neural processing of social stimuli, such as facial expressions (Black et al., 2017; Elsabbagh \& Johnson, 2007). Atypical facial expression processing could arise from an even more basic visual process: the rather detailed-oriented perception that is often observed in individuals with ASD (e.g.) Dakin \& Frith, 2005; Happé \& Frith, 2006: Vlamings, Jonkman, van Daalen, van der Gaag, \& Kemner, 2010). Previous research in 3- to 4-year-olds with ASD revealed both general enhanced brain activity in response to details as well as detail-driven facial expression processing (Vlamings et al., 2010). The current study aims to understand whether the visual abnormalities in (i) the processing of detailed information and (ii) the processing of facial expressions based on detailed information, are already present in infants with a heightened familial likelihood for an ASD diagnosis, and could thus be an endophenotype for ASD.

The detailed-oriented perception that is often observed in individuals with ASD might relate to differences in the processing of spatial frequencies (SF) (Boeschoten, Kenemans, Van Engeland, \& Kemner, 2007; Curby, Schyns, Gosselin, \& Gauthier, 2003; Deruelle, Rondan, Gepner, \& Tardif, 2004; Deruelle, Rondan, Salle-Collemiche, Bastard-Rosset, \& Da Fonséca, 2008; Vlamings et al., 2010). Spatial frequency refers to the number of cycles of luminance variations (i.e. changes between dark and light) per degree of the visual angle an object subtends at the retina and is measured in cycles per degree (cpd) of visual angle. Higher spatial frequencies (HSF, small-scale luminance variations, e.g. $>6 \mathrm{cpd}$ ) are suggested to play a central role in the encoding of detailed visual information such as sharp edges. Whereas lower spatial frequencies (LSF, largescale luminance variations, e.g. $<2 \mathrm{cpd}$ ) are suggested to be important for the processing of global configurations (Goffaux \& Rossion, 2006; Morrison \& Schyns, 2001). Figure 5.1 shows an example of faces that are filtered to contain only the HSF or LSF information. HSF and LSF are thought to be processed through different interconnected 
neural pathways (Carey \& Diamond, 1977; Johnson, 2005; Johnson, Senju \& Tomalski, 2015): it is hypothesized that HSF are carried by the slow-processing, cortical parvocellular pathway, while LSF are conveyed by the fast-processing, subcortical and cortical magnocellular pathways.

Atypical processing of SF in non-face stimuli in individuals with ASD has been shown from childhood onwards. Specifically, adolescents and adults with ASD show increased visual sensitivity to HSF information compared to neurotypical adolescents and adults (Kéïta, Guy, Berthiaume, Mottron \& Bertone, 2014). Atypical processing of HSF information is also observed in younger children with ASD, as indicated by differences in event-related potentials (ERPs; Boeschoten et al., 2007; Vlamings et al., 2010). For instance, the amplitude of the P100, an ERP component reflecting very early phases of visual processing, was found to be higher in response to HSF than LSF gratings in toddlers with ASD, while the opposite pattern was visible in toddlers in the control group (Vlamings et al., 2010). Whilst studies in children with ASD did not examine SF effects on later ERP components indexing later perceptual stages, findings in typicallydeveloping children reveal that SF content also affects later components such as the N2 (van den Boomen, Jonkman, Jaspers-Vlamings, Cousijn, \& Kemner, 2015). While these differences have been observed in preschool children, adolescents and adults, it is unclear whether SF processing is already atypical at an even younger age and at which stage of visual processing these differences can be detected. The current research is the first that explores SF processing in infants with a heightened familial likelihood for an ASD diagnosis.

The processing of facial expressions differs already between infants with higher familial likelihood for an ASD diagnosis (high-likelihood infants) and infants with lower familial likelihood for an ASD diagnosis (low-likelihood infants) (Key et al., 2015; Key \& Stone, 2012). Specifically, differences are revealed in the face-sensitive N290 and P400 ERP components, suggested to be the infant precursor of the adult N170 component (de Haan et al., 2003). Similar differences are also reported in child- and adulthood (for a review on ERP studies see Black et al., 2017). In particular, in child- and adulthood, 
group differences in the ERP responses to facial expressions are observed for the early visual P100 component as well as for the later visual face-sensitive N170 component. The atypical processing of facial expressions in ASD is suggested to be at least partly due to abnormalities in the processing of SF. For instance, children with ASD show increased use of HSF to process facial expressions compared to children without ASD (Deruelle et al., 2004; Deruelle et al., 2008; Vlamings et al., 2010). Notably, an ERP study indicated that in 3- to 4-year-olds with ASD the P100 amplitude differed between fearful and neutral expressions only when the face stimuli contained HSF (Vlamings et al., 2010). Children in the control group showed the opposite pattern: only in the LSF condition were there P100 amplitude differences between expressions. Note that no group differences were found for the later face-sensitive N170, which suggests that atypicalities in the use of SF to discriminate facial expressions appear at earlier phases of visual processing (Vlamings et al., 2010). However, it is unknown whether infants with a higher likelihood for an ASD diagnosis differ from infants with a lower likelihood in the use of spatial frequencies when processing facial expressions.

In sum, in the current study we aim to understand whether infants with highlikelihood (HL) and low-likelihood (LL) of developing ASD differ in (i) the general visual processing of HSF and LSF information; (ii) the processing of facial expressions when they selectively contain HSF or LSF information. To this end, we recorded cortical activity from 10-month-old HL and LL infants while they passively watched fearful and neutral faces, filtered to contain only HSF or LSF information. Based on previous results in toddlers with ASD and toddlers in the control group (van den Boomen et al., 2015: Vlamings et al., 2010), we hypothesize the HL group will show an atypical pattern of HSF versus LSF processing compared to the LL group regardless of the expression displayed, as indexed by the P100, N290, and P400 components.

With regard to the second aim of this study, we base our hypotheses of the LL group on recent ERP research with typically-developing 10-month-olds (van den Boomen, Munsters, \& Kemner, 2019). This study revealed that infants had differential neural responses to facial expressions when only HSF information was available, but not 
when only LSF information was available. However, the study had a slightly different design than the current one: van den Boomen and colleagues (2019) presented happy expressions in addition to the current fearful and neutral ones, and did not investigate the P100 component. Amplitude differences at the N290 and P400 components were observed between happy and fearful or neutral expressions, but not between fearful and neutral ones. As such, we do not expect differences between fearful and neutral expressions at these later components in the current study. However, it is possible that differences between these expressions can be observed at the P100 component. Therefore, we hypothesize that for the LL group, the P100 will be sensitive to type of expression when faces contain only HSF but not when faces contain only LSF. For the HL group we hypothesize, based on Vlamings and colleagues (2010), an atypical pattern of activation at the P100 component. Note that the current study is part of a longitudinal project which started before the study of van den Boomen and colleagues (2019) was published; therefore, we based our choice of manipulation of stimuli and of facial expression conditions, that is, fearful and neutral expressions, on the work done with ASD children (i.e. Vlamings et al., 2010).

\section{Materials and methods}

\section{Participants}

Thirty-eight 10-month-olds participated in this study, of which 20 had at least one older sibling with a clinical diagnosis on the autism spectrum (HL group; diagnosis confirmed to researchers via a copy of the diagnostic report), and 18 had at least one older sibling without a clinical diagnosis on the autism spectrum, and no family history of clinical diagnosis on the autism spectrum (LL group; based on parent report; see Table 5.1). All included infants were born full term ( $>36$ weeks). During the visit, the Mullen Scales of Early Learning (MSEL; Mullen, 1995) was also administered. HL and LL infants did not differ in chronological age or developmental level as measured by the MSEL Composite score (see Table 5.1). An additional fifty-two infants were excluded due to an insufficient number of valid trials due to experimental error ( 9 infants; e.g. infant looking behaviour 
was not recorded, visual task did not run properly for technical error), no EEG data being acquired because of problems with the EEG equipment or refusal of the infant to wear the EEG cap (6 infants), insufficient looking to screen (2 infants), or high level of artifacts in the EEG data (35 infants; see the preprocessing section for a detailed description of artifacts). The HL infants were recruited via collaborations with practitioners and via Dutch patients and parent associations. The LL infants were recruited from the participant databases of Utrecht University and the Radboud University Nijmegen. The current study was embedded in a longitudinal multi-centre study looking at the early development of autism (EU-AIMS project; see Loth et al., 2017). Infants were tested at one of two sites (A or B); the testing procedure was identical unless otherwise noted. The project at both sites was approved by the Medical Ethical Committee of the Arnhem-Nijmegen Region (protocol NL42726.091.13). The study was performed in accordance with the Helsinki Declaration. Parents signed informed consent prior to participation and received monetary compensation for their time, travel costs when applicable, and a small present for the child. At the time of writing, we do not have complete information on the diagnostic outcome of the sample.

Table 5.1 Characteristics of the participants included in the analysis. We verified that the groups were similar in age, number of viewed trials, and developmental stage as measured by the MSEL Composite Score.

\begin{tabular}{ccccc}
\hline & HL & LL & $t(36)$ & $p$ \\
\hline $\mathrm{N}$ & $20(8+)$ & $18(9+)$ & & .64 \\
Age in days (SD) & $308(22.6)$ & $311(16.2)$ & 0.47 & \\
Range age in days & $257-344$ & $274-345$ & & .11 \\
Viewed trials (SD) & $106(16.8)$ & $116(20.4)$ & 1.64 & .46 \\
MSEL-ELC (SD) & $92.1(12.3)$ & $94.9(10.2)$ & 0.74 & \\
\hline
\end{tabular}




\section{Stimuli}

Face stimuli were photographs of 10 models each expressing a neutral and a fearful expression, which were taken from the MacBrain Face Stimulus Set ${ }^{1}$. The pictures included 5 males and 5 females, of which 6 European-American, 3 African-American and 1 Asian-American model. Using Photoshop, face pictures were trimmed to remove external features (neck, ears and hairline), all stimuli were cropped, turned into grey scale and matched for size (19.4 x 14.0 degrees of visual angle at a viewing distance of 65 cm). All faces were filtered with a low- (LSF; < 2 cycles per degree) or high-pass (HSF; > 6 cycles per degree) spatial frequency filter. The LSF and HSF stimuli differed in terms of Root Mean Square (RMS) contrast (LSF: $25 \mathrm{~cd} / \mathrm{m}^{2}$; HSF: $8 \mathrm{~cd} / \mathrm{m}^{2}$ ). Stimuli were presented on a grey background (rgb: 131x131x131). Taken together, this created a 2 (expression: neutral, fearful) x 2 (SF: LSF, HSF) condition design (Figure 5.1).

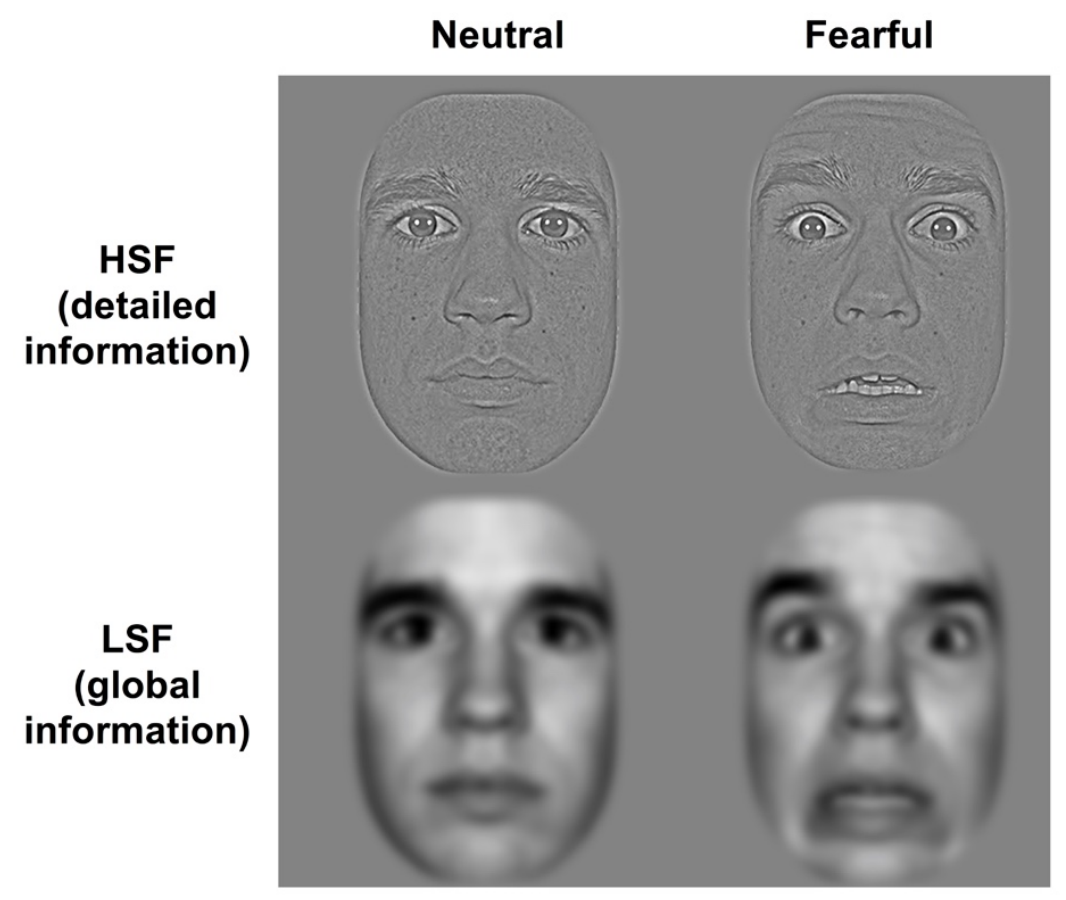

Figure 5.1 Examples of the neutral (first column) and fearful (second column) face stimuli, filtered to contain higher spatial frequency (HSF, detailed information, first row) or lower spatial frequency (LSF, global information, second row).

\footnotetext{
${ }^{1}$ Development of the MacBrain Face Stimulus Set was overseen by Nim Tottenham and supported by the John D. and Catherine T. MacArthur Foundation Research Network on Early Experience and Brain Development. Please contact Nim Tottenham at tott0006@ tc.umn.edu for information on the stimulus set.
} 


\section{Procedure}

Infants were visited at home by the research team (site A) or invited to the lab (site B). When testing took place at home (site A), a tent was placed over the dining table to approximate equal lighting conditions for each measurement. The tent comprised fabric in front, to the left and right sides, and above the child to block surrounding visual distractions. The back of the tent was left open, so parents or experimenters could move closer to the child if they were too fussy or showed discomfort. At site B, the infant and one parent were seated in a Farraday-shielded testing booth. At both sites, the infant sat on a high-chair or on the parent's lap at approximately $65 \mathrm{~cm}$ distance from a 23-inch computer monitor (refresh rate $60 \mathrm{~Hz}, 1920 \times 1080$ resolution). Parents were instructed to prevent interaction with their child as much as possible during the experiment, but to stop the child from pulling the cables. In addition, the parents were instructed that if interaction was required the parent could hold the child or the child's hand, but not stroke or bounce the child, and to respond with sentences such as 'I see the screen' but not name items on or point to the screen. The face stimuli were presented in two blocks of 20 stimuli per condition in random order for $800 \mathrm{~ms}$, with a jittered interstimulus interval (grey screen with coloured squares) between 700 and $1000 \mathrm{~ms}$. A video camera placed near the screen recorded the child's behaviour during the testing session. When the infant disengaged from the screen, the experimenter paused the task and reoriented the infant's attention by playing additional sounds or a moving stimulus on the screen. Unattended trials were discarded from analyses. The average number of attended trials was 106 for the HL group and 116 for the LL group (see Table 5.1). The experiment ended when all 160 trials were presented or until the child was too distracted or fussy to attend. The task lasted approximately $5 \mathrm{~min}$ (excluding breaks and attention reorientation). 


\section{Data analyses}

\section{ERP recording}

Two different EEG systems were used to record brain activity at the two testing-sites. At site A, EEG data was acquired using the 32-channel ActiveTwo BioSemi system (Amsterdam, Netherlands); electrodes were positioned at standard EEG recording locations according to the international 10-20 system. During the experiment, continuous EEG was acquired at a $2048 \mathrm{~Hz}$ sample rate using Actiview (version 7.05). Two electrodes, CMS (Common Mode Sense) and DRL (Driven Right Leg) provided an active ground. At site B, EEG was recorded from $32 \mathrm{AgCl}$ active electrodes in the 10-20 configuration, in a child-sized EEG cap (ActiCap, Brain Products, Munich, Germany) using BrainVision Recorder, via a BrainAmp BrainVision Products amplifier. EEG was recorded continuously, with an online reference at FCz, at a sampling frequency of $500 \mathrm{~Hz}$ and with a band-pass filter $(0.1-125 \mathrm{~Hz})$.

\section{Preprocessing}

EEG data were pre-processed using Brain Vision Analyser software (version 2.1; Brainproducts, $\mathrm{GmbH}$ ). Data were resampled offline to $512 \mathrm{~Hz}$, and filtered with a highpass filter of $0.1 \mathrm{~Hz}(24 \mathrm{~dB} / \mathrm{oct})$, a low-pass filter of $30 \mathrm{~Hz}(24 \mathrm{~dB} / \mathrm{oct})$ and a notch filter of $50 \mathrm{~Hz}$. Continuous EEG data were first divided into epochs of $100 \mathrm{~ms}$ pre-stimulus (baseline) until $1000 \mathrm{~ms}$ post-stimulus and then demeaned with baseline defined as 100 ms pre-stimulus until stimulus onset. Videos of the experimental session were manually coded for looking behaviour by a person blind to infants' likelihood of developing ASD, and trials were removed in all electrodes if the child blinked or looked away between 0 and $500 \mathrm{~ms}$ after stimulus onset. Artifacts were defined as amplitudes $+/-200 \mu \mathrm{V}$; as a difference of less than $3 \mu \mathrm{V}$ within $200 \mathrm{~ms}$; or as a voltage change of more than $50 \mu \mathrm{V}$ from the previous sampling point. An electrode was rejected if there were less than 5 artifact-free trials. We removed trials when more than 5 electrodes (16\% of the total number of electrodes) contained artifacts, based on previous research on face processing in infants (see van den Boomen et al., 2019). Finally, the activity of each electrode was re-referenced to the average of all included electrodes. 
Participants were included in the statistical analyses if the final average per experimental condition contained at least 10 trials per condition for critical electrodes (i.e., P7, P3, O1, Oz, O2, P4, P8). The average number of included trials was 24.7 per condition $(S D=0.51$, Range $=24.2-25.3)$. The total number of included trials differed between the LL and HL group (LL: $M=107.3, S D=26.1 ; \mathrm{HL}: M=91.3, S D=21 ; t(36)$ $=2.1, p=.041)$. However, it is unlikely that such difference can explain the current results. First, because the included number of trials per condition differed significantly between groups only for the Fear LSF condition $(t(36)=2.3 ; p=.029$; all other $p s>.05)$, and the difference consisted of relatively few trials (5 out of 22 to 27 trials). Next, and most importantly, because for any condition and group the included number of trials did not correlate with the amplitude of any component (all ps $>.05$ except for Fear LSF trials and P100 Fear LSF where $p=.05$ in the HL group). Thus, the number of included trials per condition did not relate to the amplitude and is therefore unlikely to explain differences in amplitude.

\section{Component analyses}

The components of interest were the P100, N290 and P400. Mean amplitude within a time window of 120-240 ms (P100), 240-340 ms (N290) and 340-600 ms (P400) was used to compute statistical analyses on these components. For each component, electrodes of interest were P7, P3, O1, Oz, O2, P4, P8; our choice of electrodes was based on previous similar research (e.g., Guy, Richards, Tonnsen, \& Roberts, 2018; Munsters, van Ravenswaaij, van den Boomen, \& Kemner, 2019; Vlamings et al., 2010) and the presence of peaks in these electrodes was confirmed by visual inspection. Mean amplitude was averaged over all electrodes of interest for each component to limit the number of statistical comparisons.

\section{Statistical analyses}

Statistical tests were performed using IBM SPSS Statistics 25.0 (IBM Corporation, Armonk, NY, USA). For the P100 amplitude, we investigated whether the brain activity of HL and LL infants differed in (i) general SF processing, and (ii) facial expression processing based on specific SF. Thus, we planned to compute a repeated measures 
ANOVA with SF (HSF vs. LSF) and Expression (neutral vs. fearful) as within-subject factors, and Group (HL vs. LL) as a between-subjects factor. Whereas an interaction between Group and SF indexes group difference in the general processing of SF (hypothesis i), a three-way interaction indicates that differential facial expression processing is based on different SF information for HL vs. LL infants (hypothesis ii).

However, one variable (i.e. the P100 amplitude evoked by LSF fearful faces in the HL group) was not normally distributed. Therefore, the results from the ANOVA provide an indication about a possible interaction but should be interpreted with caution. For this reason, we would also conduct separate tests for each hypothesis: (i) to test effects of SF on the cortical activity collapsed over facial expression (i.e. mean activity of both facial expressions) we conducted a repeated measures ANOVA with SF as within and Group as between subject variable. Because the collapsed activity over facial expressions was normally distributed in both groups, this analysis was conducted parametrically in an ANOVA that was possibly followed-up by t-tests. (ii) To test effects of facial expression for each of the SF per group we conducted Wilcoxon signed rank tests. This analysis was conducted non-parametrically for both groups. Note that even though only in the HL group the data was not normally distributed, we chose nonparametric testing for analyses on both groups instead of only the HL group to ensure equal statistical power of analyses in each group.

For the N290 and P400 components, we were only interested in group differences in SF processing. Recall that based on previous literature we did not expect any interaction between Expression and SF for these components. This was confirmed by additional analyses, reported in the Supplementary Materials IV. Thus, for each of these components we first collapsed the mean amplitude over facial expression, and then computed repeated measures ANOVAs with SF (HSF vs. LSF) as within-subject and Group (HL vs. LL) as between-subjects factors. For all reported analyses, the alpha value was set at .05. When appropriate, Bonferroni correction for multiple testing was applied. 


\section{Results}

\section{P100 amplitude}

Figure 5.2 depicts the grand averages for the P100 of the HL and LL group. The ANOVA yielded a significant 3 -way interaction $\left(F(1,36)=5.92, p=.020, \eta_{p}^{2}=.14\right)$, and a significant main effect of Expression $\left(F(1,36)=4.64, p=.038, \eta_{p}^{2}=.11\right)$. No other interaction or main effect reached significance (all $p s>.24$ ).

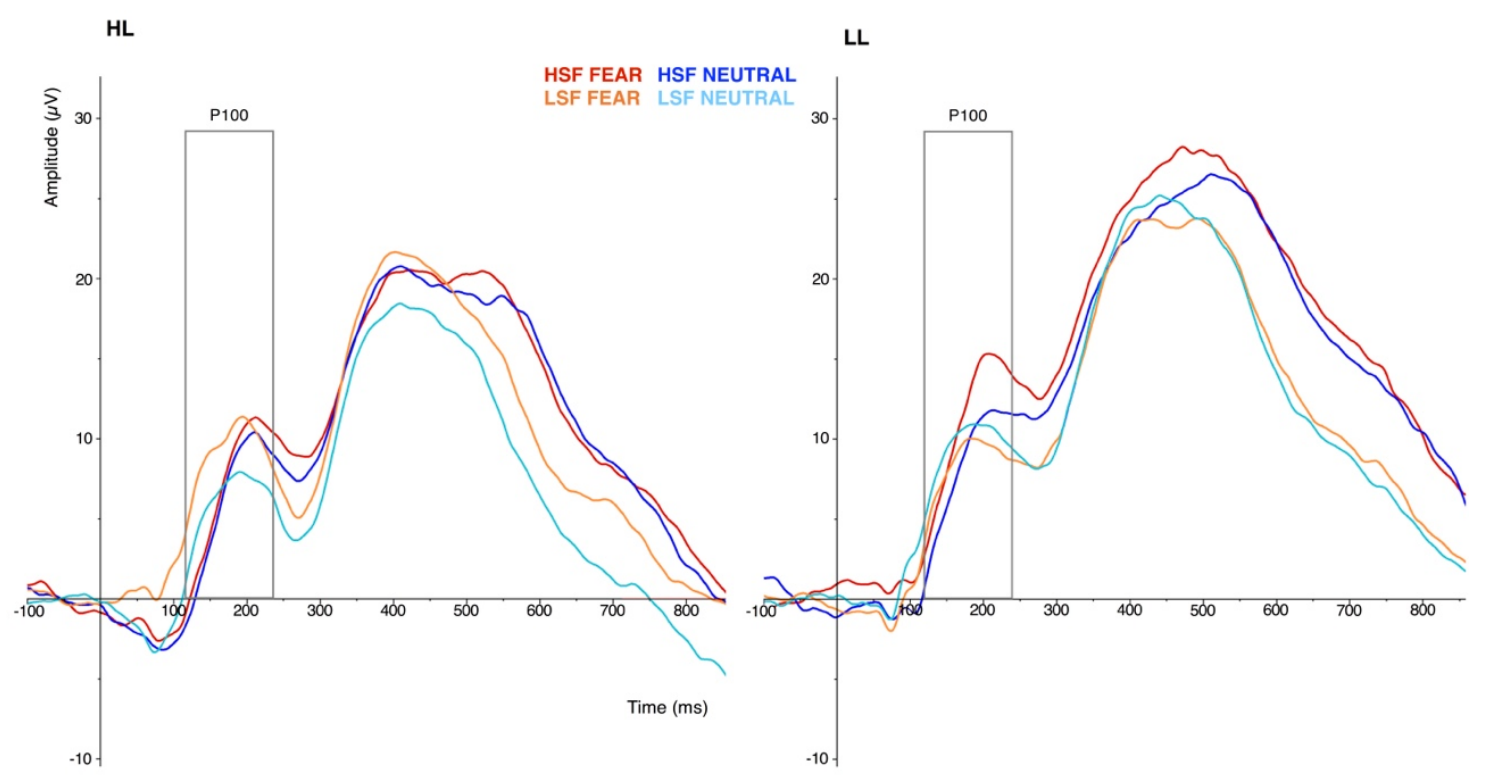

Figure 5.2 Grand averages of the HL (left panel) and LL (right panel) group, at the pooled electrodes. The grey box indicates $\mathrm{P} 100$ time window.

\section{P100 amplitude: SF processing}

There was no 2-way interaction between SF and Group $\left(F(1,36)=1.40, p=.25, \eta_{p}^{2}=\right.$ .037) indicating that there were no group differences in general SF processing at the P100 amplitude.

\section{P100 amplitude: Facial expression processing based on SF}

The analyses testing whether HL and LL infants had differential neural responses to facial expressions based on specific SF revealed that only for the LL group HSF fearful faces elicited a significantly larger P100 amplitude $(M d n=10.4)$ than HSF neutral faces $(M d n=7.90), Z=2.37, p=.018, r=.56$ (see Figure 5.3). No difference between fearful and neutral faces was found for the LL group in the LSF condition ( $M d n$ Fearful = 7.99; 


\section{Chapter 5}

$M d n$ Neutral $=9.85 ; Z=0.022, p=.98, r=.005)$, nor for the HL group in either SF condition (HSF: $M d n$ Fearful $=7.14 ; M d n$ Neutral $=6.24 ; Z=0.71, p=.48, r=.16$; LSF: $M d n$ Fearful $=7.27 ; M d n$ Neutral $=7.53 ; Z=2.02, p=.044, r=.45 ;$ against alpha of .025 corrected for two tests per group ${ }^{2}$ ).

LL

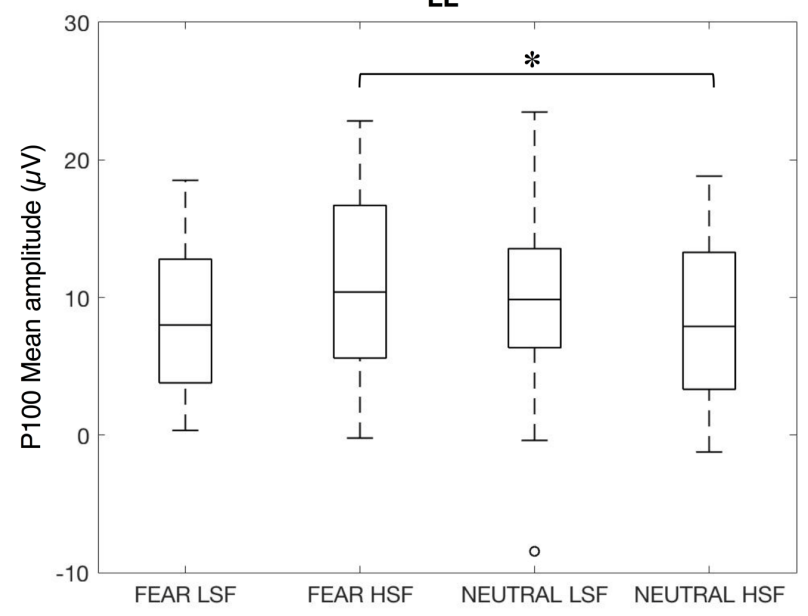

HL

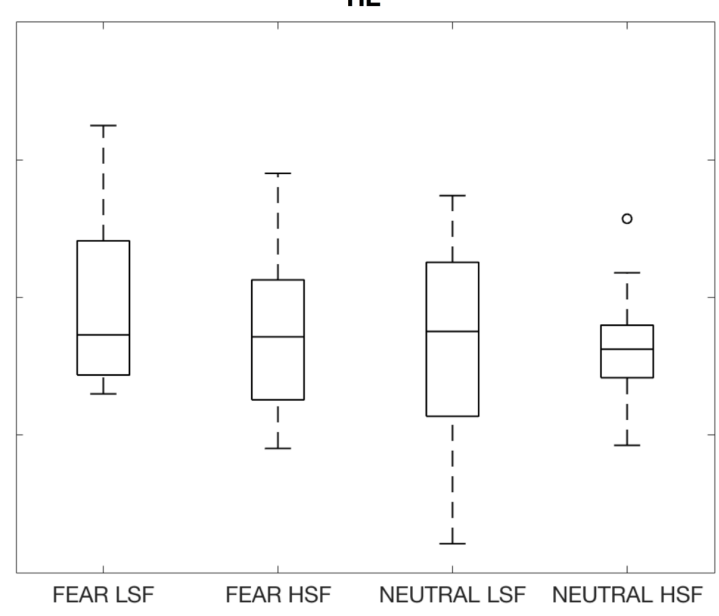

Figure 5.3 Box plots of the P100 mean amplitudes obtained for each condition in the LL (left panel) and HL group (right panel). The horizontal line in the box plots indicates the median, the two whiskers denote the $1^{\text {st }}$ and $3^{\text {rd }}$ quartile, the circles indicate outliers. The asterisks above the lines linking the different conditions indicate statistical difference (after correction).

\footnotetext{
${ }^{2}$ Note that we here corrected for 2 tests because we had a specific question for each group. When correcting for 4 tests, which corresponds to the total amount of comparisons computed in both groups, no effect reached significance. Yet, while in the discussion we interpret the results corrected for 2 comparisons, we acknowledge the weakness of the effects and are cautious in their interpretation.
} 


\section{N290 and P400 amplitudes: SF processing}

Figure 5.4 depicts the grand averages for N290, and P400.

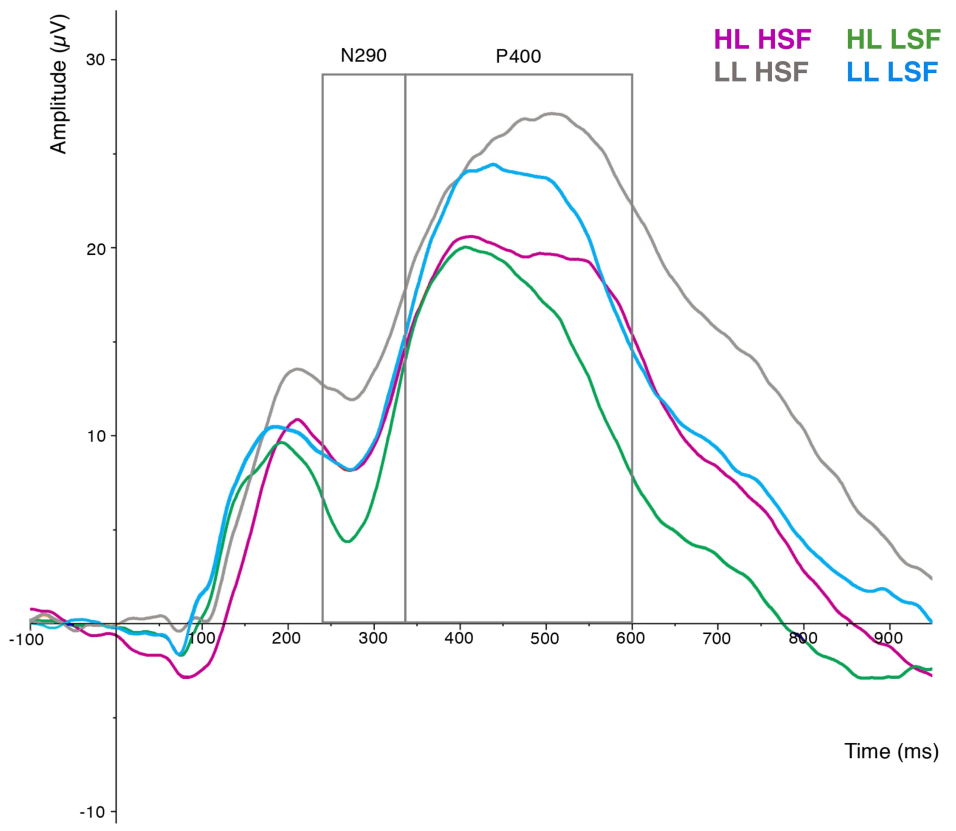

Figure 5.4 Grand averages at the pooled electrodes for the N290 and P400, relative time windows of interest are marked with grey boxes.

\section{N290 amplitude: SF processing}

There was a main effect of SF $\left(F(1,36)=10.5, p=.003, \eta_{\mathrm{p}}{ }^{2}=.23\right)$, LSF $(M=8.76$; $S D=$ $8.54)$ evoked a more negative response than $\operatorname{HSF}(M=11.8 ; S D=8.40)$. No other main or interaction effect was found (all ps > .21; see Figure 5.5).

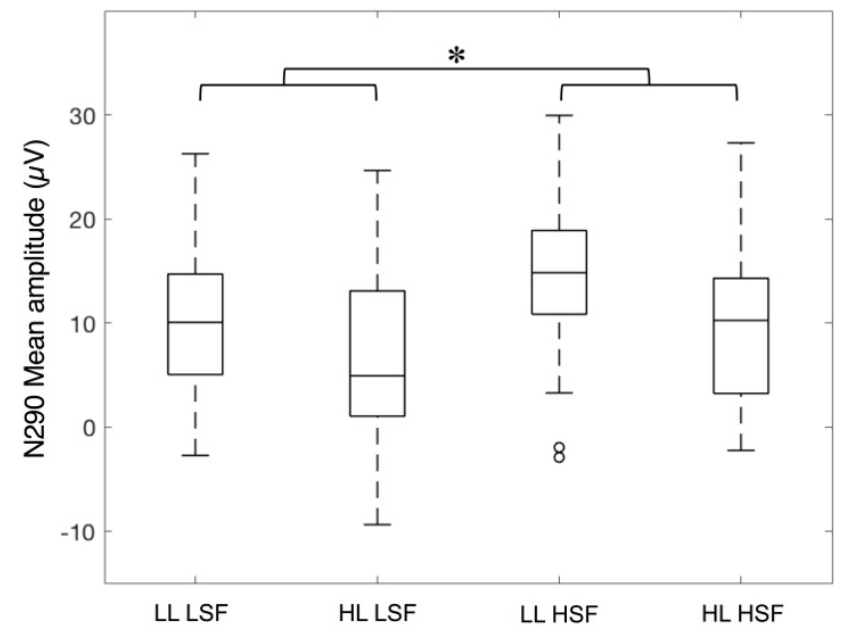

Figure 5.5 Box plots of the N290 mean amplitudes elicited by LSF and HSF stimuli, in the LL and HL group. The asterisks indicate (corrected) significant statistical differences. 


\section{P400 amplitude: SF processing}

Similarly to our findings for the N290, there was a main effect of SF $(F(1,36)=9.55, p=$ $\left..004, \eta_{\mathrm{p}}{ }^{2}=.21\right):$ LSF evoked a lower amplitude $(M=19.0 ; S D=8.91)$ than HSF stimuli $(M=21.9 ; S D=9.75)$. No main effect of Group or interaction with SF was found (all $p s$ $>.068$; see Figure 5.6).

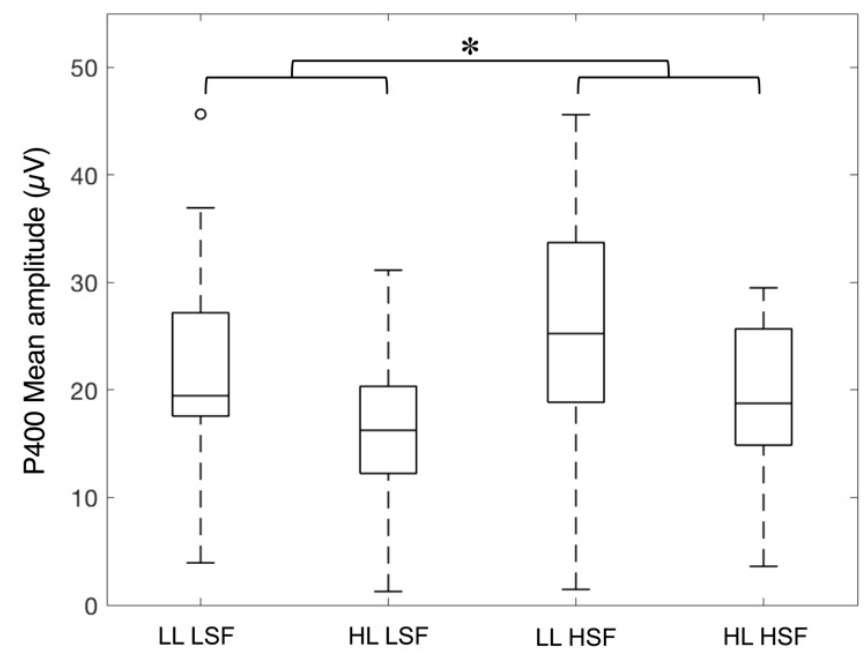

Figure 5.6 Box plots of the P400 mean amplitudes elicited by LSF and HSF stimuli, in the LL and HL group. The asterisks indicate (corrected) significant statistical differences.

\section{Discussion}

Previously, it has been suggested that the rather detailed-oriented perception that is often observed in individuals with ASD might contribute to difficulties in facial expression processing (e.g., Vlamings et al., 2010; Dakin \& Frith, 2005; Happé \& Frith, 2006). Yet, little is known on visual perceptual differences in infants with a heightened familial likelihood of developing ASD. In the present study, we examined whether 10month-old infants with a higher familial likelihood for an ASD diagnosis (HL) already differ from infants with lower familial likelihood for an ASD diagnosis (LL) in (i) the general visual processing of higher spatial frequency (HSF) and lower spatial frequency (LSF) information, and (ii) in processing facial expressions when they selectively contain HSF or LSF information. We measured visual ERPs in response to neutral and fearful facial expressions, filtered to contain only HSF or only LSF information. We analysed group differences in SF processing for the mean amplitude of each component of 
interest (i.e. P100, N290, P400) in response to all faces regardless of the emotional content. The results of these analyses revealed no difference between HL and LL infants in SF processing. That is, both groups showed similar P100 amplitudes for HSF and LSF stimuli, and lower N290 and P400 amplitudes for LSF than HSF stimuli. Crucially, we examined differences between HL and LL infants in the use of SF when processing facial expressions, as indexed by the amplitude of the early P100. Consistent with our hypothesis, our results suggest that LL infants had differential brain responses to facial expressions only when HSF were displayed, whereas the HL group had similar brain responses to fearful and neutral faces in both SF condition.

The analyses of differential neural responses to HSF versus LSF information revealed no overall differences between HL and LL infants at 10 months of age. Yet, there might be differences in the developmental trajectories between HL children who receive an ASD diagnosis later on and LL children. That is, we found that, in both infant groups, HSF elicit more positive amplitudes than LSF (i.e. in the N290 and P400 components). This pattern is similar to the cortical patterns of typically-developing infants at 1 year of age (for a review see van den Boomen, van der Smagt, \& Kemner, 2012), but opposite to 3- to 15-year-olds' patterns (van den Boomen et al., 2015; Vlamings et al., 2010). As such, children in the control (LL) group exhibit a reversal of the ERP patterns evoked by HSF and LSF between infancy and toddlerhood. However, there is no such reversal in the ERP pattern when comparing HL infants to toddlers with ASD (Vlamings et al., 2010), who both show more positive amplitudes for HSF stimuli. Possibly, the reversal of neural responses to SF in children in the control (LL) group relates to changes in visual sensitivity to SF across development: behavioural studies revealed that sensitivity to HSF develops more rapidly during the first years of life compared to LSF (for a review see van den Boomen et al., 2012). The changes in sensitivity and the reversal in neural responses might reflect a development of the neural pathways involved in HSF and LSF processing, which are suggested to be the parvocellular and magnocellular pathways, respectively (Carey \& Diamond, 1977; Johnson, 2005, 2015). The absence of a reversal in ERP patterns when comparing HL 
infants from the current study to previously studied ASD toddlers (Vlamings et al, 2010) might be seen as support for theories suggesting a disruption in these neural pathways (e.g., Johnson, 2005; Schultz, 2005). Yet, longitudinal studies following HL infants throughout the first years of life are required to confirm the absence of a reversal in ASD and to fully understand when SF processing diverges from that of typically-developing children.

Furthermore, our study suggests that HL and LL infants differ in the use of SF when processing facial expressions. Specifically, our results indicate that the neural activity of the LL infants differed between facial expressions only when images containing HSF information were displayed, whereas for the HL group there was no difference between the brain responses to fearful versus neutral faces in either SF condition. Comparing these results to the literature suggests that children with ASD have a delayed development of the use of HSF information. As hypothesized, in the LL infants, facial expression processing seemed to be driven by HSF information at very early phases of visual processing (i.e., P100). This finding is consistent with previous work on later processing stages of facial expression processing in typically-developing infants (Jessen \& Grossman, 2017; van den Boomen et al., 2019). Note that previous infant studies did not test SF effects on the P100 component. Conversely, for the HL group we found an indication of similar cortical responses to neutral and fearful faces in both SF conditions. These results provide the first evidence that HL and LL infants might differ in the processing of facial expressions when only LSF or only HSF information is available. Notably, a previous study showed that the ERP pattern of toddlers with ASD differed between facial expressions when HSF faces were displayed (P100; Vlamings et al., 2010), a pattern similar to that of the LL group of the current study. In that study, the ERP responses of toddlers in the control group differed between expressions when LSF faces were displayed (Vlamings et al., 2010). Thus, while for children in the control group facial expression processing seems to be based on HSF information at 10 months, and relies on LSF information at 3 years, differential neural responses to facial expressions seem not to be present in HL infants at 10 months and 
are based on HSF in 3-year-olds with ASD. These developmental patterns might signal delayed development of facial expression processing in ASD. A delayed development of specific processes has been previously reported in children with ASD. For instance, ERPs evoked by faces in 18- to 30-month-olds with ASD resembled ERPs observed in typically-developing 12- to 17-month-olds, but differed from age-matched typicallydeveloping children (Webb et al., 2011). It is unknown whether these delays in development resolve, such that processing of faces and facial expressions differs less from typically-developing individuals later on in development. Prior research with HL infants suggests that delayed development is resolved in some domains in ASD (e.g., grasping and reaching, Libertus, Sherperd, Ross \& Landa, 2014; face processing, Jones et al., 2016) or that the presence of differences fluctuates across development (e.g., work on motor delays by Estes et al., 2015). Thus, it is difficult to predict whether a delayed development in facial expression processing will turn into a typical use of LSF information to process facial expressions later on individuals with ASD.

A delay in the development of processing facial expressions filtered to contain only HSF or LSF information early in life might affect the efficiency of expression processing. This could be due to the neural pathways via which HSF and LSF are processed in the brain (Johnson, 2005; 2015). Recall the slow-processing cortical parvocellular route is thought to carry mainly HSF information, whereas the fast-processing subcortical and cortical magnocellular routes are thought to operate on LSF information. Thus, using HSF information might lead to slower facial expression processing than using LSF information. As such, if the neural responses differ between facial expressions only when HSF information is presented, as observed in toddlers with ASD (Vlamings et al., 2010), the result might be slower facial expression processing. The possible slower processing could also impact other aspects of social development, e.g. social interaction. Notably, a widely accepted account of human brain development referred to as neuroconstructivism posits that seemingly small differences early in life could accumulate into cascading effects in several domains over development (D'Souza \& Karmiloff-Smith, 2016). For example, the later onset of the ability to efficiently process 


\section{Chapter 5}

facial expressions (i.e. via LSF information) might lead to difficulties in interpreting the social environment and consequently to differences in social behavior. In turn, this could affect the behavior of parents and peers, providing the child with different social input. Eventually, this cascade of alterations could contribute to difficulties in effective social communication.

While interpreting the current results, one should take into account that no information on diagnostic outcome is currently available for our sample. Consequently, one should not interpret our results as early signs of ASD since only a part of the HL group (approximately 20\%, cf. Ozonoff et al., 2011) will develop ASD. Rather, our results indicate that differences in processing facial expressions when only LSF or HSF information is available could be an endophenotype of ASD, that is, an example of a subclinical autism trait observed at a neurocognitive level. Future research should test whether the differences reported here are evident in those HL infants who do later receive a diagnosis of ASD. In addition, future studies should follow HL infants across the first years of life to understand how sensitivity to SF in facial expressions as well as in non-social stimuli develops in ASD, and whether atypical SF processing affects later social development. Furthermore, only limited conclusions can be drawn regarding face processing of real-life faces. The current stimuli were filtered to contain specific spatial frequency information and therefore different from real-life, unfiltered, faces. Moreover, manipulating spatial frequencies resulted in changes in luminance contrast of the stimuli as well. However, a prior study with adults (i.e. Vlamings et al., 2009) used stimuli in which the luminance contrast was either equalized or not equalized across SF conditions, and showed that the facial expression effects were not driven by the luminance contrast differences between the HSF and LSF conditions. It is worth mentioning that we choose not to correct our stimuli for luminance because we aimed at keeping the experiment as similar as possible to that of Vlamings and colleagues (2010), i.e. prior work that tested SF effects on facial expression processing in older children with and without ASD, in which stimuli were also not equalized for luminance. It should be noted however that both contrast sensitivity and spatial frequency 
processing develop throughout childhood. Therefore, possible effects of contrast differences between spatial frequency conditions in the current study cannot be excluded.

Overall, the current study showed that HL and LL infants do not differ in general SF processing, but they seem to differ in the processing of facial expressions when only LSF or HSF information is available. That is, unlike LL infants, HL infants seemed to have similar ERP responses to both facial expressions when only LSF or HSF information is available. Adding the current results to previous findings in toddlers with ASD (Vlamings et al., 2010) suggests a developmental delay in efficient processing of facial expressions in ASD.

\section{Acknowledgements}

This research has received support from the Innovative Medicines Initiative Joint Undertaking under grant agreement $\mathrm{n}^{\circ} 115300$ (EU-AIMS), resources of which are composed of financial contribution from the European Union's Seventh Framework Programme (FP7/2007 - 2013) and EFPIA companies' in kind contribution. This research is further supported by a grant from the European Commission's Horizon 2020 Program under Grant Agreement $n^{\circ} 642996$ (BRAINVIEW) (RDL, EW), by the Netherlands Organization for Scientific Research (NWO) VICI grant n ${ }^{\circ} 45307004$ (CK), and the K.F. Hein Fonds (CB). We thank all the infants and families that took part in the research. We also thank the research assistants at site A (Lilli van Wielink, Karlijn Blommers, and Evelien Klijn) and at site B the research assistants (Loes Vinkenvleugel, Manon De Korte, Nicole Driessen, Annemiek Kruit), and students (Wendy Visser and Dewy Nijhof), who helped with data collection, and researchers Ricarda Braukmann and Mirjam Pijl who set up the study and implemented the task at site B; Roy Hessels who programmed the task script. We also thank Jan Buitelaar and Sabine Hunnius as PIs responsible for the research conducted at site B. 


\section{References}

American Psychiatric Association. (2013). Diagnostic and statistical manual of mental disorders (5th ed.). Washington, D.C.: Author.

Black, M. H., Chen, N. T. M., Iyer, K. K., Lipp, O. V, Bölte, S., Falkmer, M., ... \& Girdler, S. (2017). Neuroscience and Biobehavioral Reviews Mechanisms of facial emotion recognition in autism spectrum disorders: Insights from eye tracking and electroencephalography. Neuroscience and Biobehavioral Reviews, 80, 488-515. http://doi.org/10.1016/j.neubiorev.2017.06.016

Boeschoten, M. A., Kenemans, J. L., Van Engeland, H., \& Kemner, C. (2007). Abnormal spatial frequency processing in high-functioning children with pervasive developmental disorder (PDD). Clinical Neurophysiology, 118(9), 2076-2088. http://doi.org/0.1016/j.clinph.2007.05.004

Carey, S., \& Diamond, R. (1977). From piecemeal to configurational representation of faces. Science, 195(4275), 312-314.

Curby, K.M., Schyns, P.G., Gosselin, F., \& Gauthier, I. (2003). Differential use of spatial frequency scales for face recognition in a person with Asperger's syndrome. Journal of Vision, 3(9), 821.

de Haan, M., Johnson, M. H., \& Halit, H. (2003). Development of face-sensitive event-related potentials during infancy: a review. International Journal of Psychophysiology, 51(1), 4558 .

D'Souza, H., \& Karmiloff-Smith, A. (2016). Neurodevelopmental disorders. Wiley Interdisciplinary Reviews: Cognitive Science, 8(1-2), e1398. http://doi.org/10.1002/wcs.1398

Dakin, S., \& Frith, U. (2005). Vagaries of visual perception in autism. Neuron, 48, 497-507. http://doi.org/10.1016/j.neuron.2005.10.018

Deruelle, C., Rondan, C., Gepner, B., \& Tardif, C. (2004). Spatial frequency and face processing in children with autism and Asperger syndrome. Journal of autism and developmental disorders, 34(2), 199-210.

Deruelle, C., Rondan, C., Salle-Collemiche, X., Bastard-Rosset, D., \& Da Fonséca, D. (2008). Attention to low- and high-spatial frequencies in categorizing facial identities, emotions and gender in children with autism. Brain and Cognition, 66(2), 115-123. http://doi.org/10.1016/j.bandc.2007.06.001

Elsabbagh, M., \& Johnson, M. H. (2007). Infancy and autism: progress, prospects, and challenges. Progress in Brain Research, 164, 355-383. http://doi.org/10.1016/S00796123(07)64020-5 
Estes, A., Zwaigenbaum, L., Gu, H., John, T. S., Paterson, S., Elison, J. T., ... \& IBIS network. (2015). Behavioral, cognitive, and adaptive development in infants with autism spectrum disorder in the first 2 years of life. Journal of neurodevelopmental disorders, 7(1), 24. http://doi.org/10.1186/s11689-015-9117-6

Goffaux, V., \& Rossion, B. (2006). Faces are "spatial" - holistic face perception is supported by low spatial frequencies. Journal of Experimental Psychology. Human Perception and Performance, 32(4), 1023-1039. http://doi.org/10.1037/0096-1523.32.4.1023

Guy, M. W., Richards, J. E., Tonnsen, B. L., \& Roberts, J. E. (2018). Neural correlates of face processing in etiologically-distinct 12-month-old infants at high-risk of autism spectrum disorder. Developmental cognitive neuroscience, 29, 61-71. https://doi.org/10.1016/j.dcn.2017.03.002

Happé, F., \& Frith, U. (2006). The weak coherence account: detail-focused cognitive style in autism spectrum disorders. Journal of autism and developmental disorders, 36(1), 5-25. http://doi.org/10.1007/s10803-005-0039-0

Jessen, S., \& Grossmann, T. (2017). Exploring the role of spatial frequency information during neural emotion processing in human infants. Frontiers in human neuroscience, 11, 486. http://doi.org/10.3389/fnhum.2017.00486

Johnson, M. H. (2005). Subcortical face processing. Nature Reviews Neuroscience, 6(10), 766774. http://doi.org/10.1038/nrn1766

Johnson, M. H., Senju, A., \& Tomalski, P. (2015). The two-process theory of face processing: modifications based on two decades of data from infants and adults. Neuroscience \& Biobehavioral Reviews, 50, 169-179. https://doi.org/10.1016/j.neubiorev.2014.10.009

Jones, E. J., Venema, K., Earl, R., Lowy, R., Barnes, K., Estes, A., ... \& Webb, S. J. (2016). Reduced engagement with social stimuli in 6-month-old infants with later autism spectrum disorder: a longitudinal prospective study of infants at high familial risk. Journal of neurodevelopmental disorders, 8(1), 7. http://doi.org/10.1186/s11689016-9139-8

Kéita, L., Guy, J., Berthiaume, C., Mottron, L., \& Bertone, A. (2014). An early origin for detailed perception in Autism Spectrum Disorder: biased sensitivity for high-spatial frequency information. Scientific reports, 4, 5475. https://doi.org/10.1038/srep05475

Key, A. P., \& Stone, W. L. (2012). Same but different: 9-month-old infants at average and high risk for autism look at the same facial features but process them using different brain mechanisms. Autism Research, 5(4), 253-266. https://doi.org/10.1002/aur.1231

Key, A. P., Ibanez, L. V., Henderson, H. A., Warren, Z., Messinger, D. S., \& Stone, W. L. (2015). Positive affect processing and joint attention in infants at high risk for autism: an exploratory study. Journal of autism and developmental disorders, 45(12), 4051-4062. https://doi.org/0.1007/s10803-014-2191-x 
Libertus, K., Sheperd, K. A., Ross, S. W., \& Landa, R. J. (2014). Limited fine motor and grasping skills in 6-month-old infants at high risk for autism. Child development, 85(6), 2218-2231. https://doi.org/10.1111/cdev.12262

Loth, E., Charman, T., Mason, L., Tillmann, J., Jones, E. J., Wooldridge, C., ... \& Banaschewski, T. (2017). The EU-AIMS Longitudinal European Autism Project (LEAP): design and methodologies to identify and validate stratification biomarkers for autism spectrum disorders. Molecular Autism, 8(1), 24. doi: 10.1186/s13229-017-0146-8

Morrison, D. J., \& Schyns, P. G. (2001). Usage of spatial scales for the categorization of faces, objects, and scenes. Psychonomic Bulletin \& Review, 8(3), 454-469. http://doi.org/10.3758/BF03196180

Mullen, E.M. (1995). Mullen scales of early learning. Circle Pines, MN: American Guidance Service, Inc.

Munsters, N.M., van Ravenswaaij, H., van den Boomen, C., \& Kemner, C. (2019). Test- retest reliability of infant event related potentials evoked by faces. Neuropsychologia, 126, 2026. http://doi.org/ 10.1016/j.neuropsychologia.2017.03.030

Ozonoff, S., Young, G. S., Carter, A., Messinger, D., Yirmiya, N., Zwaigenbaum, L., ... \& Hutman, T. (2011). Recurrence risk for autism spectrum disorders: a Baby Siblings Research Consortium study. Pediatrics, 128(3), e488-e495. https://doi.org/10.1542/peds.2010-2825

Schultz, R. T. (2005). Developmental deficits in social perception in autism: the role of the amygdala and fusiform face area. International Journal of Developmental Neuroscience, 23(2-3), 125-141. https://doi.org/10.1016/j.ijdevneu.2004.12.012

van den Boomen, C., Jonkman, L. M., Jaspers-Vlamings, P. H., Cousijn, J., \& Kemner, C. (2015). Developmental changes in ERP responses to spatial frequencies. PloS one, 10(3), e0122507. https://doi.org/10.1371/journal.pone.0122507

van den Boomen, C., Munsters, N. M., \& Kemner, C. (2019). Emotion processing in the infant brain: The importance of local information. Neuropsychologia, 126, 62-68. http://doi.org/10.1016/j.neuropsychologia.2017.09.006

van den Boomen, C., van der Smagt, M. J., \& Kemner, C. (2012). Keep your eyes on development: the behavioral and neurophysiological development of visual mechanisms underlying form processing. Frontiers in Psychiatry, 3, 16. http://doi.org/10.3389/fpsyt.2012.00016

Vlamings, P. H. J. M., Goffaux, V., \& Kemner, C. (2009). Is the early modulation of brain activity by fearful facial expressions primarily mediated by coarse low spatial frequency information? Journal of Vision, 9(5), 12.1-13. http://doi.org/10.1167/9.5.12 
Vlamings, P. H. J. M., Jonkman, L. M., van Daalen, E., van der Gaag, R. J., \& Kemner, C.

(2010). Basic abnormalities in visual processing affect face processing at an early age in autism spectrum disorder. Biological Psychiatry, 68(12), 1107-1113.

http://doi.org/10.1016/j.biopsych.2010.06.024

Webb, S. J., Jones, E. J., Merkle, K., Venema, K., Greenson, J., Murias, M., \& Dawson, G. (2011). Developmental change in the ERP responses to familiar faces in toddlers with autism spectrum disorders versus typical development. Child development, 82(6), 18681886. http://doi.org/10.1111/j.1467-8624.2011.01656.x 


\section{Supplementary Materials IV}

In this supplementary file we report analyses on the possible effects of facial expression, spatial frequencies (SF), and genetic likelihood of ASD for the N290 and P400 amplitudes. We provide these additional analyses as supplementary material as these still might be of interest for developmental researchers.

Specifically, for each visual component we performed a repeated measures ANOVA with SF (HSF vs. LSF) and Expression (neutral vs. fearful) as within-subject factors, and Group (HR vs. LR) as a between-subjects factor. Significant three-ways interactions are followed-up with simpler two-ways ANOVA split by Group. Note that here we report only main or interaction effects with Expressions, as all other effects are reported in the main text.

\section{N290}

There was no main effect or interaction with Expression, all $p$ s $>.14$.

\section{P400}

There was a significant interaction between Expression, SF and Group $(F(1,36)=4.23$, $\left.p=.047, \eta_{\mathrm{p}}^{2}=.10\right)$. We followed the three-way analyses with two-way ANOVAs testing per group a possible interaction between SF and Expression. This interaction was not significant in either the HL or LL group (HL: $F(1,19)=2.44 ; p=.135 ; \mathrm{LL}: F(1,17)=1.91$; $p=.185)$. Therefore, we also investigated main effects of Expression per group, which were not significant either (HL: $F(1,19)=2.27 ; p=.15 ; \mathrm{LL}: F(1,17)=0.27 ; p=.61)$. Thus, there was no Group x Expression effect, and no main effect of Expression at the P400 component.

Together these results support previous findings with typically developing 10-montholds (van den Boomen et al., 2019), by showing for both likelihood groups no difference in the neural responses to fearful and neutral expressions in any SF condition for either visual component, i.e. N290 and P400. 


Chapter 6

Summary and General Discussion 
Being able to discriminate faces from other objects or to tell apart emotional facial expressions are crucial skills for effective social communication at any stage of life, but especially for young infants whose communication is essentially non-verbal. While previous research with adults identified the neural networks underlying face and facial expression processing, the developmental literature still struggles in providing a clear picture of how these networks unfold in infancy. The overarching aim of my thesis is to contribute to our understanding of how the infant brain develops face and facial expression processing skills between the first and second semester of life. My research focused on this period as behavioural evidence suggests it to be pivotal for the development of these abilities (Figure 6.1).

To date, fNIRS and ERPs are the preferred techniques to infer infant brain responses to faces. Previous fNIRS and ERPs studies provide some indication of how face and facial expression processing develop in the first year of life. Specifically, face processing appears to be already available in the first semester of life and it possibly recruits parts of the same neural network involved in adult face processing (e.g., Nakato et al., 2009). Facial expression processing, on the other hand, seems to emerge between the first and second semester of life (Leppänen \& Nelson, 2009). By seven months after birth, the encoding of emotional facial expressions involves temporal and frontal regions, which are also part of the adult emotion processing network (Fox, Wagner, Shrock, Flusberg, \& Nelson, 2010; Nakato, Otsuka, Kanazawa, Yamaguchi, \& Kakigi, 2011). Whereas these studies suggest that face and facial expression processing become functional in the first year of life, the literature is not consistent in reporting when and how these processing skills develop in infancy. Also, we know very little about which factors contribute to individual differences in face and facial expression processing. It is fundamental to identify such factors if we want to define the range of typical development; in turn such knowledge will aid the early detection of infants deviating from this range.

Therefore, one aim of my thesis is to contribute in defining how face and facial expression processing develop in the first year of life, using fNIRS and ERPs (Figure 6.1 
provides a summary of the research questions relative to this aim). In particular, Chapter 3 and 4 seek to answer questions on the development of face processing: (i) Which cortical area is involved in face processing at five months of age (Chapter 4)?; (ii) Does face processing further develop between five and 10 months of age (Chapter 3)? Whereas Chapter 4 and 5 answer questions relative to facial expression processing: (i) Can the infant brain discriminate between facial expressions already at five months of age? If so, which cortical area responds to emotional expressions (Chapter 4)? (ii) Are the genetic likelihood for autism spectrum disorder (ASD) and the visual processing of specific visual information (i.e. facial details and global information) factors contributing to individual differences in facial expression processing at 10 months of age (Chapter 5)?

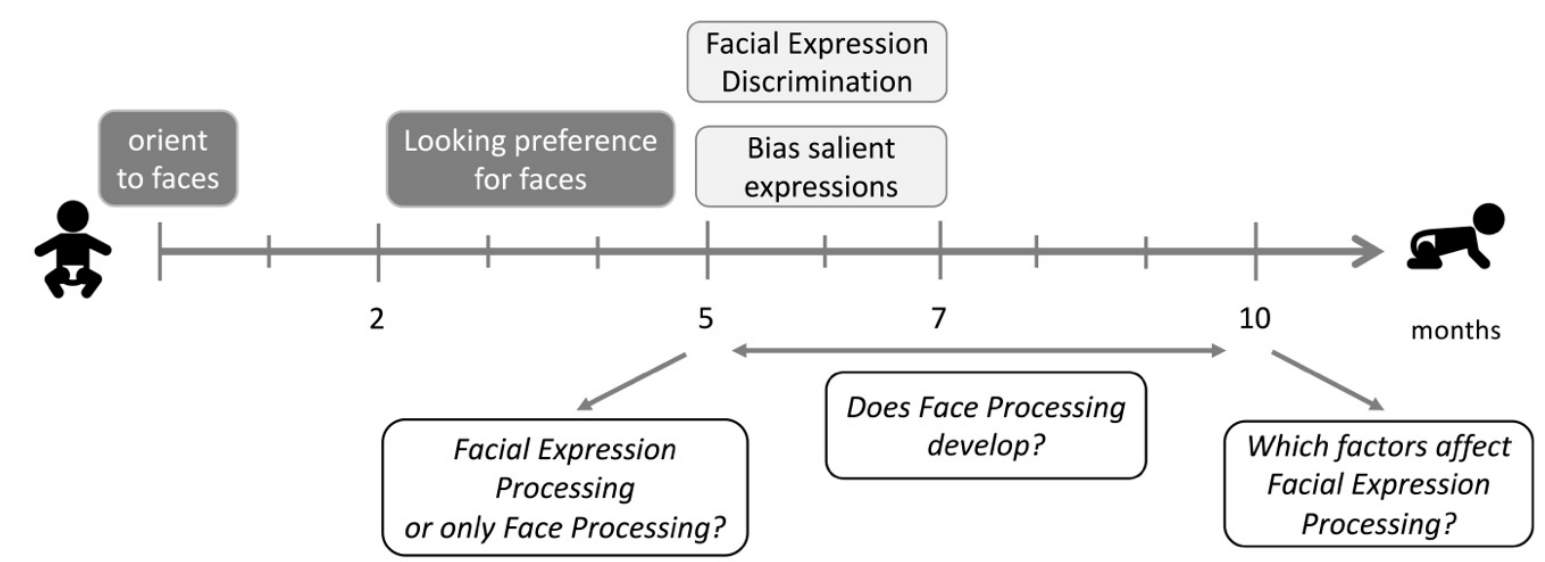

Figure 6.1 Scheme of previous behavioral findings for face and facial expression processing within the first year of life (boxes above the time line). The white boxes below the time line represent the questions of this thesis.

As already mentioned in this thesis' introduction, although fNIRS and ERPs have been used for several decades in infant research, the field lacks consensus on how to measure robust neural markers of face processing in infancy. Yet, identifying and agreeing on such robust neural markers are essential steps to make valuable neuroscientific interpretations of research findings as well as to draw direct comparisons between different studies. Thus, another aim of my research is to contribute in reaching such consensus among labs by addressing two pending questions in this field of 
research: (i) What is the optimal approach to correct for motion artifacts in infant fNIRS data (Chapter 2)?; (ii) Which of the ERP components previously defined as face-related are robust markers of face processing across infancy (Chapter 3)?

In the following paragraphs I will summarize and discuss the experimental findings of this thesis by first focusing on the methodological aims, and then turning to the more developmentally-oriented aims. I will also acknowledge potential limitations and suggest future directions for each aim.

\section{Part 1 Methods:}

\section{How do we obtain robust measures of infant face processing?}

\section{fNIRS: What is the optimal approach to correct for motion artifacts in infant data?}

\section{Summary of results \& discussion}

Chapter 2 investigated which approach is optimal for the correction of motion artifacts in infant fNIRS data. To this end, we first compared the independent performance of most of the currently available motion correction techniques and of a novel approach (Spline + Wavelet combined) on a semi-simulated infant dataset (i.e. containing the true/simulated HRF). Next, we tested the performance of Spline, Wavelet, and their combination on three different infant task-based datasets. Given that infant data are quite noisy, we hypothesized that the stacking of two different correction techniques (i.e. Spline + Wavelet) would have a better performance in motion artifact correction compared to their individual use or to other single techniques. The selection of Spline and Wavelet was based on previous recommendations for motion artifact correction in adult data (Brigadoi et al., 2014; Cooper et al., 2012), which is typically less noisy than infant data. The analyses of Chapter 2 revealed two main findings.

First, the approaches with the worst performance were those with either full rejection of trials affected by motion artifacts (Rejection) or those with inclusion of all trials regardless of the amount of motion artifacts (No Correction). Specifically, Rejection and No Correction performed poorly in recovering the true HRF in semisimulated data (RMSE metric) as well as in reducing the variability of the HRFs within 
and between subjects in the three task-based datasets (within- and between-subjects standard deviation metrics). Also, Rejection recovered less than $50 \%$ of the presented trials in the three task-based datasets. It is essential to recover an adequate number of trials to obtain reliable measures of brain activation. These findings align with previous studies on adult and infant data, which also discourage Rejection (Behrendt et al., 2018; Brigadoi et al., 2014). Possibly, Rejection can be efficient when recordings are contaminated with only a few isolated artifacts and include a high number of trials, but this is not a common situation in infant studies. This first set of results is critical since Rejection is currently widely used as main correction approach in infant research. Thus, fNIRS infant researchers should always correct for motion artifacts rather than reject trials in infant data.

A second key finding of this work highlights that the proposed combined approach Spline + Wavelet was the most effective in (i) recovering the true HRF in infant semisimulated data when data contained low or high percentages of artifacts; (ii) reducing the variability of the responses within- and between-subjects; (iii) recovering almost all trials affected by artifacts in all the three task-based datasets. Therefore, for infant data containing a large number of motion artifacts we suggest the combined use of Spline and Wavelet. In addition, we tested both possible orders of Spline and Wavelet and suggest to use Spline followed by Wavelet as this order reported a slightly better recovery of the true HRF in the semi-simulated dataset (i.e., lowest RMSE median). Together these results indicate Spline + Wavelet as optimal for effective motion artifact correction in noisy infant data. Given that the efficacy of Spline + Wavelet has been proven on four infant datasets that differed on a number of levels (i.e. data acquired from different sites, using different paradigms, age groups, NIRS systems, with levels of noise) our recommendation can be safely generalized to any infant dataset or to any dataset containing a large amount of noise (e.g. clinical data).

While we suggest the use of Spline + Wavelet, we advise infant researchers to critically evaluate the parameters set to detect motion artifacts in the recordings, i.e. parameters in the Homer2 function hmrMotionArtifactByChannel. This step is 
required before Spline can be applied, and critically influences the efficacy of Spline artifact correction. Indeed, the selection of inappropriate parameters to detect motion artifacts might compromise the efficacy of data cleaning, and consequently impact the shape of the haemodynamic response. In particular, we suggest careful tuning of the STDEVthresh parameter. This parameter allows the detection of the signal exceeding a threshold in change of standard deviation. In Chapter 2, we set this threshold by visually evaluating the efficacy of the function in identifying the major motion artifacts present in the data. As one can note, this procedure is time consuming and highly subjective. Thus, we encourage future research to implement a more automatic and objective procedure for motion detection in noisy (infant) data.

An alternative option to avoid such subjective decision, is to use Wavelet alone. This technique proved to be quite efficient in correcting moderately noisy recordings, and to perform better than other single techniques (e.g. Spline). The main benefit of Wavelet is that it does not require a prior motion detection step; consequently, it might be more suitable for researchers novel to infant NIRS data and for the processing of large datasets. It is noteworthy that also Wavelet requires the tuning of a parameter, i.e. the interquartile range (iqr). The choice of iqr value depends on the timing of the task design (i.e. length of stimuli presentation) and therefore on the characteristics of the HRF. For example, choosing a too low iqr might reduce or filter out the HRF in event-related designs. To aid researchers in the choice of such parameter we also compared the effect of a selection of $i q r$ values (i.e. $0.1,0.5,0.8,1.0$ and 1.5) on three types of HRF, each simulating the HRF resulting from different task designs. The results suggest to use an $i q r=0.5$ when analysing data collected with stimuli of $10 \mathrm{~s}$ or longer, and an $i q r=0.8$ for data collected with stimuli shorter than $10 \mathrm{~s}$.

In sum, the findings of Chapter 2 recommend developmental researchers to first evaluate the amount of noise contained in the data and use (a) Spline + Wavelet combined for very noisy data or (b) Wavelet for data with a moderate percentage of artifacts. Results of this work will improve the standardization of preprocessing pipeline 
in infant fNIRS studies, and hopefully make results more reliable and comparable among different research groups.

\section{Future directions and further considerations}

Chapter 2 focused on improving the step of motion artifact correction, but there are other steps in the preprocessing stream that warrant further analysis. For instance, the band-pass filter is commonly used to remove irrelevant frequencies associated with physiological oscillations (e.g. slow systemic changes, heartbeat, breathing, sucking on a pacifier). Yet, the incorrect tuning of the band-pass thresholds might undermine or filter out the HRF. Indeed, the frequency of the HRF (determined by the stimulus presentation) might be close to the frequency of unwanted oscillations. Furthermore, some of these oscillations (e.g., heart rate) change with development, so longitudinal studies might require different filtering per age point or a less stringent filter. Hence, care should be taken when choosing the filter values to avoid removing relevant neural signal. Future studies should investigate which is the most appropriate, and systematic, way to choose such filtering thresholds (for further discussion see Pinti, Scholkmann, Hamilton, Burgess, \& Tachtsidis, 2018) as well as which combination of motion correction technique and band-pass filter best suits infant fNIRS data.

Another important challenge in infant fNIRS research is the understanding of unclear patterns of infant haemodynamic responses. Indeed, several infant studies report cases of inverted $\mathrm{HRF}$ (i.e. a decrease of $\mathrm{HbO}$ coupled with an increase in $\mathrm{HbR}$ ) or simultaneous change in $\mathrm{HbO}$ and $\mathrm{HbR}$ (e.g. see Figure 4.4B, brain responses to happy faces, which depicts atypical HRF). The physiology or the cognitive basis of these patterns is still unclear. It has been suggested that these non-canonical patterns might be dependent on specifics of the experimental design adopted as well as on the developmental stage of the cortical areas of interest (for a review see Issard \& Gervain, 2018). It is essential to understand what triggers such non-canonical shapes before we can fully interpret such effects within developmental context. Another choice to consider refers to the design of the experiment. A recurrent advice in the developmental fNIRS community consists in the careful selection of the baseline stimuli. Indeed, ideally 
Chapter 6

baseline stimuli should elicit no or a less strong activation in the area of interest compared to the experimental stimuli. In the opposite case, a local decrease in oxygenation during the experimental trial might occur. Thus, while infant fNIRS researchers should carefully design their task and preferably pilot novel paradigms, further research should investigate how changes in the brain maturation contribute to the variability in infant haemodynamic responses.

In Table 6.1 I summarized the main recommendations for infant fNIRS study design and data preprocessing. I based such recommendations on previous literature and on my personal lab experience. 
Table 6.1 Recommendations for fNIRS study design and data preprocessing

\begin{tabular}{|c|c|c|}
\hline & & Recommendations \\
\hline \multirow{6}{*}{$\begin{array}{l}\text { Task } \\
\text { Design }\end{array}$} & $\begin{array}{l}\text { Type of } \\
\text { Design }\end{array}$ & $\begin{array}{l}\text { Block design is more common than event-related design because it } \\
\text { allows for HRFs with greater amplitudes (i.e. easier to pick-up effects). } \\
\text { It is important to make tasks infant friendly! }\end{array}$ \\
\hline & $\begin{array}{l}\text { Length of } \\
\text { trials }\end{array}$ & $\begin{array}{l}\text { Experimental trial: long enough to elicit a response, min. 2s. Baseline } \\
\text { trials: (a) In block designs: long enough to allow HRF to return to a } \\
\text { baseline level. Jittering the experimental trial onset by varying the } \\
\text { duration of the baseline trials avoids physiological and anticipatory } \\
\text { effects. (b) In event-related designs the response to a stimulus overlaps } \\
\text { with the response to the next stimulus (this is resolved using GLM). } \\
\text { Note: observed HRF is dependent on the length of both experimental } \\
\text { and baseline trials. Pilot new paradigms or use previously tested } \\
\text { designs. (see Gervain et al., 2011; Lloyd-Fox et al., 2010). }\end{array}$ \\
\hline & $\begin{array}{l}\text { Baseline } \\
\text { stimulus }\end{array}$ & $\begin{array}{l}\text { Should allow the ROI to 'rest', i.e. stimuli trigger no activation, or at } \\
\text { least lower than the exp. trials, from the ROI. In block designs baseline } \\
\text { trials should precede and follow the exp. trials. }\end{array}$ \\
\hline & $\begin{array}{l}\text { Number } \\
\text { of trials }\end{array}$ & $\begin{array}{l}\text { Should be a compromise between the SNR, habituation effects, and } \\
\text { subject comfort. } 3 \text { trials per condition is the minimum used for } \\
\text { statistical analyses (e.g., Lloyd-Fox et al., 2013). }\end{array}$ \\
\hline & $\begin{array}{l}\text { Position } \\
\text { Channels }\end{array}$ & $\begin{array}{l}\text { Channel position should refer to external landmarks on the scalp or a } \\
\text { co-ordinate system (e.g. 10-20 system). If participants' anatomical MRI } \\
\text { are available, measurement points can be mapped to underlying cortical } \\
\text { regions; otherwise reference to an age-appropriate brain template is } \\
\text { preferable (Lloyd-Fox et al., 2014). }\end{array}$ \\
\hline & $\begin{array}{l}\text { Selection } \\
\text { of ROIs }\end{array}$ & $\begin{array}{l}\text { Should be based on previous literature and include a brain area that does } \\
\text { not respond to the experimental manipulation as control. }\end{array}$ \\
\hline \multirow{5}{*}{$\begin{array}{l}\text { Pre- } \\
\text { processing } \\
\text { (based on } \\
\text { Chapter 2) }\end{array}$} & $\begin{array}{l}\text { Discard } \\
\text { invalid } \\
\text { trials }\end{array}$ & $\begin{array}{l}\text { For instance, non-looking trials. Usually these trials are the most } \\
\text { affected by motion artifacts. }\end{array}$ \\
\hline & $\begin{array}{l}\text { Pruning } \\
\text { channels }\end{array}$ & $\begin{array}{l}\text { Discard channels with very low or high light intensity levels. Intensity } \\
\text { ranges are dependent on the system used. }\end{array}$ \\
\hline & $\begin{array}{l}\text { Motion } \\
\text { artifact } \\
\text { detection }\end{array}$ & $\begin{array}{l}\text { As initial parameters use, in Homer2, tMotion }=1 \text {; tMask }=1 \text {; } \\
\text { STDEV thresh }=13-15 ; \text { AMPthresh }=0.4 \text { ). Although it is important to } \\
\text { fine-tune these parameters based on the noise in each sample. }\end{array}$ \\
\hline & $\begin{array}{l}\text { Motion } \\
\text { artifact } \\
\text { correction }\end{array}$ & $\begin{array}{l}\text { Spline }+ \text { Wavelet for very noisy infant data. Wavelet for moderately } \\
\text { noisy data, use an iqr }=0.5 \text { for trials of } 10 \mathrm{~s} \text { or longer; iqr }=0.8 \text { for data } \\
\text { collected with stimuli shorter than } 10 \mathrm{~s} \text {. }\end{array}$ \\
\hline & $\begin{array}{l}\text { Band-pass } \\
\text { filter }\end{array}$ & $\begin{array}{l}\text { The low cut-off should be set considering the stimulus presentation } \\
\text { timing, to avoid removing the HRF. The high cut-off should reduce low } \\
\text { frequency noise; if finely tuned signal-detrending is not required. }\end{array}$ \\
\hline
\end{tabular}


ERPs: Which of the ERP components previously defined as face-related are robust markers of face processing across the first and second semester of life?

\section{Summary of results \& discussion}

In Chapter 3, I used a large longitudinal dataset to test which of the ERP components often reported as face-related (i.e. N290, P400, Nc) are robust markers of face processing across infancy. Face processing here is intended as differential brain activity between face and other stimuli (i.e. houses). A robust ERP marker of face processing should show evidence of face processing in a high proportion of 5-month-olds, coupled with a high proportion of those infants still showing this pattern at 10 months, and few showing alternative trajectories. These criteria were based on previous behavioral evidence which consistently reports face discrimination in infants older than three months of age (cf. Libertus, Landa, \& Haworth, 2017); trajectories alternative to that of the dominant group are interpreted mainly as atypical trajectories, possibly as the result of measurement error.

The group level analyses (ANOVAs) revealed that all ERP components (i.e. N290, $\mathrm{P} 400, \mathrm{Nc}$ ) detect face-house differential cortical activity at five and at 10 months of age. More specifically, the amplitudes of the N290 and the P400 components were more negative in response to faces than houses, whereas the opposite pattern was found for the Nc. Note that I will refer to these patterns as dominant when describing the results of the individual level analyses. The group results could suggest that all three components are robust markers of infant face processing. Yet, the analyses at the individual level (Markov model) depict a different scenario. Indeed, using Markov models, the N290/P400 components revealed more robust activations across infancy than the Nc. Specifically, compared to the Nc, the N290/P400 models displayed: (1) a higher proportion of infants showing the dominant face processing pattern at five months (N290/P400 > 83\%; $\mathrm{Nc}=60 \%$ ); (2) a higher proportion of these 5-month-olds still presenting the same dominant response at 10 months $(\mathrm{N} 290 / \mathrm{P} 400>85 \%$; $\mathrm{Nc}=$ 67\%); (3) a higher proportion of infants moving from a non-dominant pattern of face processing at five months to the dominant pattern at 10 months (N290/P400 $=67-93 \%$; 
$\mathrm{Nc}=53 \%)$. The longitudinal pattern described at point 3 might be explained by measurement error or by developmental delay, i.e. infants who develop face processing skills later than at five months of age (for more discussion on this point see the following section on face processing development). Therefore, the results of Chapter 3 suggest that the N290/P400 components more consistently measure face processing across infancy compared to the Nc.

Why do we observe such differences in robustness between the N290/P400 and the Nc components? A possible explanation lies in the different mechanisms that these components are thought to reflect. The N290/P400 components have been often linked to the early stages of visual processing of faces (for reviews see de Haan, Johnson, \& Halit, 2003; Nelson \& McCleery, 2008). It is likely that the extensive exposure to faces that infants experience from early on contributes in making these visual components sensitive to faces from few months after birth and in retaining such sensitivity throughout the first year of life. This line of thought is in accordance with an influential developmental model of face processing, which posits that cortical specialization emerges as the result of infants' increased experience with faces, while it is also influenced by intrinsic biases to orient to faces and mechanisms of inter-regional connectivity (Johnson, Senju, \& Tomalski, 2015). The Nc, on the other hand, has been associated with a number of higher-level mechanisms; for instance, it is thought to reflect allocation of attention, preference, or familiarity (for a review see de Haan et al., 2003). It is possible that these mechanisms competed with each other over the course of our experiment and across the two visits. In other words, the Nc might not only pick up on the general face-house contrast, but might also fluctuate as it is sensitive to familiarity or novelty of the stimuli. Therefore, the underlying mechanisms of the Nc may be less steady over time or across individuals compared to the visual processing indexed by N290/P400.

It is worth noting that the Markov model is a descriptive approach that depends on the a-priori defined thresholds used to calculate group-membership (i.e. an absolute face-house amplitude difference above $1.5 \mu \mathrm{V}$ was classified as a significant difference). 
While these thresholds were defined by a researcher expert in infant face processing who was blind to the study results, we cannot exclude that a different choice of thresholds would have presented a lower variability within and between visits for the Nc. Yet, visual inspection of individual grand-averaged responses confirmed the difference observed between the Markov models of the N290/P400 and the Nc. Further, our results are in line with a recent infant ERP study reporting a substantial test-retest reliability for the N290, while only a moderate reliability for the Nc component (Munsters, van Ravenswaaij, van den Boomen, \& Kemner, 2019). Thus, our findings strongly suggest that the N290/P400 patterns differ from the Nc in terms of robustness.

Another important point of discussion concerns our choice of dependent variable: the mean amplitude. It could be argued that the latency rather than amplitude could possibly be a better marker of face processing. Indeed, previous studies showed that the P400 latency shares some characteristics with the adult face-sensitive N170. For instance, similarly to the adult N170, the infant P400 latency has been found faster for faces compared to other objects (e.g., de Haan \& Nelson, 1999; Jones et al., 2016). However, we decided not to test the robustness of the latency of the P400, nor of the other components, as the components in our study were rather broad waves instead of clear peaks. Therefore, it was not possible to reliably determine the peak latency for all conditions in most infants (but see in the Supplementary Materials II tests on the N290 latency in response to faces, which is a measure that could be clearly identified).

To summarize, Chapter 3 indicates that, compared to the Nc, the N290/P400 components are more robust markers of face processing between five and 10 months of age. This finding suggests a difference in the underlying mechanisms. The results of Chapter 3 contribute to the current understanding of the role of such components in face processing, which is essential to make reliable inferences about the normative development of such processing.

\section{Future directions and further considerations}

Whereas the results of this study suggest the perceptual N290/P400 components are robust markers of face processing, these two components showed some small 
differences. Specifically, the P400 Markov model charted more alternative trajectories (as in different from the dominant trajectory from five to 10 months) than the N290 model. Whereas the N290 model displayed only alternative trajectories moving to the dominant face-house difference (negative difference) at 10 months, in the P400 model there were some infants who also moved from a no face-house difference at five months to a positive face-house difference at 10 months. Given that these components are thought to reflect the same processing (i.e. visual processing), are recorded from the same sets of (occipital) electrodes, but are sampled from subsequent time-windows, the difference in alternative trajectories between the components might be explained by differences in their underlying neural generators. Indeed, a previous cross-sectional study with 4.5- to 7.5-month-olds indicated that while the generators of N290 mainly encompass temporal regions (e.g., parts of the fusiform gyrus, FG), the generators of P400 are similar to those of the Nc and include temporal as well as frontal regions (e.g. orbitofrontal gyrus; Guy, Zieber, \& Richards, 2016). Because the N290 shares the polarity (negative), the direction of sensitivity to faces (more negative amplitude for faces vs. objects), and partially the generators (e.g. FG) of the adult face-sensitive N170, Guy and colleagues suggested the N290 as only precursor of the N170. Note that to perform source-localization with EEG a minimum of 64 electrodes is desirable (DeBoer, Scott, \& Nelson, 2007), whereas studies in this thesis sampled brain activity from 32channel systems; hence we did not to include such analyses in Chapter 3. While our results corroborate the hypothesis of Guy and colleagues, further longitudinal research with high-density EEG systems encompassing a wider age-range is needed to fully understand how (or which of) these components develop into the adult N170 and whether the components show differences in their underlying generators across development.

Another cue for future research would be testing the robustness of the N290/P400 components at an earlier age. According to an accepted developmental account, face processing is initially mediated by a subcortical pathway and later also by a cortical pathway (Johnson, 2005, 2015). Recall that ERPs mainly pick up on cortical processing. 
Yet signs of cortical specialization have been reported by a PET study in infants as young as with two months (Tzourio-Mazoyer et al., 2002) and by an fNIRS study with newborns (Farroni et al., 2013). Such findings suggest that the cortical pathway already starts to be active at an earlier age than five months after birth. Thus, it is possible that the N290 and the P400 might consistently pick up face processing before five months; alternatively, these components might show a larger variability between infants possibly signalling differences in the degree of cortical specialization. Therefore, longitudinal studies should test if the N290 and the P400 prove to be robust measures of face processing before five months of age.

\section{Part2 Development:}

\section{How does the infant brain process faces and facial expressions between five and 10 months of age?}

In this section I will discuss and integrate the findings relative to infant face and facial expression processing reported in Chapter 3, 4, and 5. Specifically, I will first describe the results relative to face processing (Chapter 3 and 4) and next move to the results linked to facial expression processing (Chapter 4 and 5).

\section{Face Processing}

\section{Summary of results \& discussion}

In Chapter 4, I investigated which cortical area is involved in face processing at five months of age, by means of fNIRS. Specifically, I recorded haemodynamic activity elicited by pictures of faces, and houses as baseline stimuli, from three cortical regions of the right hemisphere: occipital (close to the OFA), temporal (close to the STS), and frontal areas (close to the OFC). Face-specific activity was expected at temporal and/or occipital areas based on previous fNIRS studies that tested a similar age group (Blasi et al., 2007; Nakato et al., 2009) as well as on previous adult models of face processing (e.g., Haxby et al., 2002). The results showed that faces elicited a significant increase in $\mathrm{HbO}$ 
from baseline only at occipital sites. Overall this finding suggests that the (cortical) face processing network is partially active in 5-month-olds.

The activity observed at occipital regions in this study is in line with a previous fNIRS study reporting face specific activity at occipital regions in 4-months-olds (Blasi et al., 2007). Likewise, another study with 5-month-olds showed that neutral faces evoke $\mathrm{HbO}$ increases in channels placed between T6 and O2 (Nakato et al., 2009), note that this scalp-location overlaps with that of our occipital channels in Chapter 4. Whereas Nakato and colleagues (2009) attribute such activation to the nearby region STS, we believe that our occipital channels are sampling activity from the OFA. Indeed, channel scalp-location in our study was based for the occipital regions on an fMRI atlas of 3- to 17-week-old infants (selection of channels close to OFA Kabdebon et al., 2014), while for the temporal region on anatomical maps of 4- to 6-month-olds derived from a NIRSMRI co-registration study (selection of channels close to STS, Lloyd-Fox et al., 2014). The increased activation for faces found at OFA in 5-month-olds is consistent with the activation found from the same area in adults (Haxby et al., 2002). Indeed, in adults this region shows increased activation for faces vs. other stimuli and is thought to be involved in the early perception of facial features (for a review on fMRI adult studies see Haxby et al., 2002). Overall, Chapter 4 replicates previous findings confirming the involvement of occipital cortical areas in face processing at five months of age. In turn, the face-specific activation at occipital channels suggests that the cortical network involved in adult face processing is partially active at this age.

Evidence corroborating the presence of neural specialization for face processing in 5-month-olds comes from the ERP study described in Chapter 3. More specifically, at this age, the amplitude of the face-related ERP components (i.e. N290, P400, Nc) discriminated faces from houses. Chapter 3 sought to investigate whether face processing further develops between the first and second semester of life, by testing the same infant sample at five and 10 months of age with the same experimental paradigm. Because previous behavioural studies indicate the second semester of life as pivotal for face processing, e.g. infants can now discriminate faces based on emotion or ethnicity 
(Anzures, Quinn, Pascalis, Slater, \& Lee, 2010; Leppänen \& Nelson, 2009), we hypothesized that also basic face processing abilities (i.e. discrimination of faces vs. houses) might further develop between the first and second semester of life. The group level analyses (ANOVAs) showed that the ERP amplitudes of all components tested discriminated between faces and houses equally at five and 10 months of age.

While this ERP result suggests a lack of change in face processing across infancy, we do not know whether there is a change in the brain areas recruited by such processing. Indeed, due to the low spatial resolution of the EEG method, it is difficult to infer the source of scalp-recorded electrical potentials (Luck, 2014). Therefore, it is possible that within this age range there is a change in the brain regions recruited in face processing or in the strength of their response to faces. For instance, Chapter 4 suggests that at five months of age the OFA is involved in face processing, while other fNIRS studies with older infants suggest face-specific activity from the temporal region STS as well (for a review see Otsuka, 2014). According to a current developmental view, experience with faces substantially contributes to the gradual cortical specialization for face processing (Johnson et al., 2015). Possibly, increased experience with faces after five months of age prompts also temporal areas to emerge as face-specific. Therefore, while the ERP evidence shown in Chapter 3 indicates no change in brain responses to faces between five and 10 months of age, we cannot exclude that the exposure to faces during this period did not influence the specialization of the cortical network involved in face processing. Future longitudinal studies combining ERPs with fNIRS should test whether the lack of change in the ERPs sensitivity to faces observed in Chapter 3 also reflects the stability of the cortical network recruited for face processing in infancy. Combining different measures of brain activation is a powerful tool to elucidate what typical development of face processing entails, which in turn provides a baseline to understand atypical development.

Another interesting result of Chapter 3 comes from the Markov Model, i.e. the descriptive approach tracing face processing from five to 10 months for each child. While in the previous paragraph on methods I discussed how this model aids the 
definition of robustness of the ERP components as markers of infant face processing, here I focus on the longitudinal trajectories that deviated from the trajectory showed by the dominant group (i.e. the majority of the sample). Recall that, for each ERP component, the dominant group showed the same face-house response pattern at five as well as 10 months: for the $\mathrm{N} 290$ and P400 components faces elicited a more negative amplitude than houses, while for the Nc the opposite pattern was described. Trajectories deviating from this longitudinal dominant pattern (e.g. a negative face-house difference at five turns into no face-house difference at 10 months) might be well explained by measurement error. However, it is possible that a portion of these alternative trajectories mirrors individual differences in face processing development. For instance, the Markov model relative to the P400 component showed that some infants do not discriminate faces from houses at five months but they do at 10 months (i.e. at 10 months they show the dominant face processing pattern). This trajectory might indicate that some infants simply require more experience with faces to develop such neural discrimination. If this is true, this result might indicate a delay in the neural specialization for face processing in some infants.

What could a delay in the neural specialization for face processing entail? According to a current developmental account, the so-called neuroconstructivism, even small differences early in life may lead to cascading effects in several domains over development (Karmiloff-Smith, 2015). Hence a delay in the development of face processing at five months might cause difficulties in other domains later in development, such as in social communication. Delays in face processing have been observed in autism spectrum disorder (ASD), a developmental disorder characterized by impairments in social communication, repetitive behaviours and restricted interests (American Psychiatric Association, 2013). Notably, it has been recently proposed that early delays in basic face processing contribute to delineate the atypical trajectories of social-communication abilities in young children with ASD (Webb, Neuhaus, \& Faja, 2017). Delays in certain aspects of face processing (i.e. familiar vs. unfamiliar faces) have been reported for the N290 latency and the Nc amplitude of 18- to 30-month-olds with 
ASD, who showed comparable responses to typically developing 12- to 17-month-olds (Webb et al., 2011). Furthermore, another study with younger infants showed that 6month-olds who later developed ASD had faster P400 latency to faces, as well as smaller and shorter Nc response to faces (vs. objects) compared to infants with a higher genetic likelihood for ASD who did not develop ASD (Jones et al., 2016); crucially these differences were only apparent at six but not at 12 months of age. Taken together these findings suggest that the alternative trajectories described in Chapter 3 might reflect developmental delays in face processing, which might be linked to later differences in social behaviour. As the data used in Chapter 3 come from a dataset which also includes behavioural measures of social competence, future research should test whether the infants showing a delayed face processing also display behavioural differences at a later age.

To summarize, the results of Chapter 3 and 4 show that face processing recruits (occipital) cortical regions at five months of age and possibly remains equally functional between the first and second semester of life, at a group level. Interestingly, descriptive analyses at the individual level suggest that there might be differences between infants in the development of face processing across the first and second semester of life; such hypothesis is supported by previous studies with infants at high likelihood for ASD (e.g. Jones et al., 2016; Webb et al., 2011).

\section{Future directions and further considerations}

While Chapter 4 tested cortical activation specific to (static) faces in 5-month-olds, a developmental model on face processing suggested that cortical specialization to faces emerges around two months of age, prior to then face processing is mainly mediated by subcortical mechanisms (Johnson, 2001, 2005; Johnson, Dziurawiec, Ellis, \& Morton, 1991). Interestingly, a recent fNIRS study with newborns indicates cortical activation specific to (dynamic) faces already shortly after birth (1- to 5-day-olds; Farroni et al., 2013), suggesting an earlier involvement of cortical areas in face processing. It is important to note, however, that such activation was elicited in only one temporal channel by dynamic videos of faces involving also social movements (i.e. eye gaze to 
right or left; mouth motion; hand motion) which might contribute to stimulate temporal regions; indeed near STS regions in adults are activated by static faces as well as by eye, mouth, hand movements (e.g. Allison, Puce, \& McCarthy, 2000). Hence it is unclear if the cortical activation described by Farroni and colleagues (2013) is face-specific or is modulated by face-motion. Therefore, it would be interesting for future research to investigate with static faces when the cortical network devoted for face processing comes online as well as whether and how cortical activation changes over the first semester of life.

This suggestion brings me to a second consideration on the results of Chapter 3, i.e. the longitudinal ERP study. The group analyses revealed no change in face processing between five and 10 months of age. Based on what discussed in the previous paragraph, it is possible that we would observe changes in ERPs from an earlier age than five months. The earliest evidence of ERP differences between faces and other objects comes from a study with 3-month-olds (Halit, Csibra, Volein, \& Johnson, 2004). It would be interesting to see whether and how the N290, the P400 and the Nc components report changes in face processing between three and five months of age, possibly in the direction of an increased specialization for faces. For now, we can conclude that there is no development in face-sensitive infant components from the first to second semester of the first year of life.

\section{Facial expression processing}

\section{Summary of results \& discussion}

Chapter 4 tested whether the infant brain shows evidence of cortical specialization for facial expression processing at five months and which cortical area is involved in such processing. We hypothesized that, if the emotion processing network is active already at five months of age, we would observe emotion-specific activity from temporal and/or frontal sites, as suggested by fNIRS studies with older infants (Fox et al., 2013; Nakato et al., 2011). The results of Chapter 4 indicate that the network is still immature. Specifically, signs of discrimination between emotional facial expressions have been 
reported at temporal regions, while no emotion effect was found at occipital or frontal regions. Further analyses revealed that happy facial expressions elicited a significant decrease in $\mathrm{HbO}$ at temporal sites, while $\mathrm{HbO}$ concentrations followed the opposite direction in the fearful condition (although not significantly above zero).

While the haemodynamic patterns observed at temporal regions do not reflect the typical responses commonly interpreted as brain activation (i.e. significant increase in $\mathrm{HbO}$ ), we report differential activity in response to fearful and happy faces. Hence, although we cannot draw any strong conclusions based on this result, we speculate that the 5-month-old brain might just have started to acquire emotion processing skills. Nevertheless, the facial emotion processing network shows immaturity. This suggestion is in line with findings of some ERPs studies (Hoehl \& Striano, 2010; Peltola, Leppänen, Mäki, \& Hietanen, 2009) and with a current developmental model on facial expression processing which suggests that the emotion processing network emerges between five and seven months of age (Leppänen \& Nelson; 2009). The immaturity of neural discrimination between facial expressions described in Chapter 4 might indicate that the infant brain requires more experience with different facial expressions before it specializes in their processing. While our study hints in the direction of a beginning of specialization, the field requires longitudinal studies including older children to elucidate when the network becomes fully functional.

Facial expression discrimination has been consistently reported by ERP studies with infant older than seven months of age (for a review see van den Boomen, Munsters, \& Kemner, 2019). This evidence suggests that at this age the infant brain holds a certain degree of specialization for facial expression processing (Leppänen \& Nelson, 2009). Hence, in Chapter 5, I tested individual differences in facial expression processing of 10month-olds, age at which neurotypical children should be able to discriminate between different emotional facial expressions. In particular, I evaluated whether infants with a higher likelihood of developing ASD (HL) differed from low-likelihood infants (LL) when processing facial expressions (fearful, neutral) filtered to contain either visual details (higher-spatial frequencies; HSF) or global information (i.e. lower-spatial 
frequencies; LSF). These analyses focused on the early perceptual P100 component as a previous study observed atypical P100 amplitudes to filtered facial expressions in toddlers with ASD (Vlamings, Jonkman, van Daalen, van der Gaag, \& Kemner, 2010). Given that a previous ERP study with neurotypical 10-month-olds indicated that facial expression discrimination at this age relies on HSF (van den Boomen et al., 2019), we hypothesized that LL infants would show discrimination between emotional expressions when faces contained only HSF, while the HL group would show atypical ERP patterns. The results support our hypotheses: (1) P100 amplitude of LL infants differed between facial expressions when only HSF were displayed; (2) P100 amplitude of HL infants showed no difference between facial expressions in either spatial frequency condition (HSF nor LSF).

The first result suggests that neurotypical 10-month-olds base facial expression discrimination mainly on the HSF contained in faces, but not on the LSF. This finding is consistent with previous work on later processing stages (i.e. N290; P400) of facial expression discrimination in typically-developing infants (Jessen \& Grossman, 2017; van den Boomen et al., 2019). Note that previous infant studies did not test SF effects on the P100 component. Therefore, we add to the current infant literature that the spatial frequency content of faces might influence infants' facial expression discrimination at even an earlier stage of visual processing.

Crucially, HL infants did not show facial expression discrimination in either spatial frequency conditions. These results indicate that HL and LL infants differ in the processing of facial expressions when only LSF or only HSF information is available. Interestingly, a previous study showed that the P100 amplitude of 3-4-year-olds with ASD differed between facial expressions when HSF faces were displayed (Vlamings et al., 2010), a pattern similar to that of the LL group of Chapter 5. At that age, toddlers without ASD showed P100 amplitude differences in response to facial expressions when only LSF faces were displayed (Vlamings et al., 2010). Therefore, while for controls facial expression discrimination seems to be based on HSF information at 10 months, and on LSF information at three years, facial expression discrimination is not present in HR 
infants at 10 months and is based on HSF in 3-year-olds with ASD. As such, these developmental patterns might signal a delay in the development of facial expression processing in ASD.

Developmental delays in ASD have been documented for face processing as well as in other domains. For instance, as previously mentioned, a previous study found that the ERPs evoked by faces in 18- to 30-month-olds with ASD resembled ERPs observed in typically-developing 12- to 17-month-olds, but differed from age-matched controls (Webb et al., 2011). Currently we do not know whether these developmental delays eventually resolve, such that face and facial expression processing become similar to that of typically-developing individuals later on in development. Indeed, while some studies with HL infants suggests that delayed development is resolved in some domains in ASD (e.g., grasping and reaching, Libertus, Sherperd, Ross \& Landa, 2014; face processing, Jones et al., 2016), others indicate that group differences fluctuate across development (e.g., work on sensorimotor delays by Estes et al., 2015). Therefore, based on the results of Chapter 5 and of the work done by Vlamings and colleagues (2010) we cannot predict whether a delayed development in facial expression discrimination will turn into a typical use of LSF information to process facial expressions later on in individuals with ASD.

It is likely that an early delay in the use of specific SF to discriminate facial expressions affects the efficiency of expression processing. This could be due to the neural pathways via which HSF and LSF are processed in the brain (Johnson 2005; 2015): HSF are carried by the slow-processing, cortical parvocellular pathway; while LSF are conveyed by the fast-processing, subcortical and cortical magnocellular pathways. Therefore, relying on HSF information might lead to slower facial expression discrimination than if one were to rely on LSF information. Consequently, HL infants not only seem to be delayed in expression discrimination, but those who later develop ASD also are delayed in the use of information that aids fast discrimination in childhood (LSF). Possibly such delays signal an early disruption in the pathways subserving LSF processing (Johnson, 2005). These early delays could also impact other aspects relevant 
for social development. As argued by the neuroconstructivism account, seemingly small differences early in life could accumulate into cascading effects in several domains over development (D'Souza \& Karmiloff-Smith, 2016). As such, the later onset of the ability to process facial expressions might lead to difficulties in interpreting the social environment, which could contribute to differences in social behaviour. This in turn might also affect the behaviour of parents and peers, who might provide a different social input to the child. Ultimately, this cascade of alterations could contribute to difficulties in effective social communication, which is a core sign of ASD (American Psychiatric Association, 2013).

It is worth mentioning that no information on diagnostic outcome was available for the infant sample tested in Chapter 5. Consequently, one should not interpret the results on HL infants as early signs of ASD since only a part of the HL group are expected to develop ASD (approximately 20\%, cf. Ozonoff et al., 2011). Rather, the results of Chapter 5 suggest that differences in the processing of facial expressions when only LSF or HSF information is available could be an endophenotype of ASD, i.e. a subclinical trait of ASD observed at a neurocognitive level. It is important for future studies to test whether the differences reported in Chapter 5 are evident in those HL infants who later receive a diagnosis of ASD. Future studies should also test whether these neurophysiological markers of atypical visual processing in infancy correlate with the severity of behavioural ASD-symptoms later in life.

In sum, Chapter 4 and 5 provide useful evidence about how the infant brain develops the ability to process facial expressions. Specifically, while Chapter 4 indicates that the neural network supporting facial expression processing is still immature at five months, Chapter 5 confirms its functionality at 10 months of age. At this age, infants seem to rely on the visual details within faces to discriminate between facial expressions. Notably, the genetic likelihood of developing ASD seem to influence such ability in 10month-olds. 


\section{Future directions and further considerations}

While Chapter 4 (fNIRS study) suggests that 5-month-olds might not be able yet to discriminate between emotional facial expressions (fearful vs. happy), previous behavioural research provided contrasting evidence. For instance, at this age infants show categorial perception of (levels of) happy faces (e.g., Bornstein \& Arterberry, 2003), and show categorical boundary between emotional expressions within the same valence (e.g. positive: happiness vs. surprise; negative: sadness vs. anger; White et al., 2019). How can we explain such discrepancy between neuroimaging and behavioural results? One possibility is that 5 -month-olds can discriminate between facial expressions, but recruit brain structures that cannot be assessed with fNIRS. Indeed, in adults, facial expression processing involves areas such as the fusiform gyrus and the amygdala, which lie too deep into the brain to be tested with fNIRS; recall that fNIRS can only record haemodynamic activity few centimetres into the cortex (Lloyd-Fox et al., 2010). Another possibility could be that the infant brain cannot discriminate between happy and fearful faces yet, but can discriminate between other emotion contrasts. While we want to generalize when the infant brain can discriminate between facial expressions, expression-discrimination effects could also be dependent on the type of emotions displayed. Hence, it might be too simplistic to assume that infant brain is suddenly capable of achieving all possible discriminations. To disentangle when and how infants become able to process facial expressions, future research should follow-up infants within the first year of life, combining behavioural and brain measures, and using different emotion contrasts (e.g. within valence and between valence).

Chapter 5 dived into individual differences in facial expression processing and suggested that infants at high and low likelihood for ASD differ in the processing of facial expressions filtered to contain only high or low spatial frequencies. However, the filtering of spatial frequencies produced changes in luminance contrast of the stimuli as well. It has been shown that such change in contrast does not influence the effect of spatial frequencies on facial expression processing in adults (Vlamings, Goffaux, \& Kemner, 2009). While this evidence suggests that changes in contrast might not have 
affected the results of Chapter 5, we cannot exclude such influence since contrast sensitivity as well as spatial frequency processing change across development (see van den Boomen, van der Smagt, \& Kemner, 2012). Therefore, it would be interesting for future studies to replicate the findings of Chapter 5 while controlling for changes in luminance contrast.

In addition, as mentioned in the Discussion section above, the lack of facial expression processing in HL infants might result into cascading effects in other domains. Hence, future perspective studies should test whether this difference in neural processing is linked to certain behavioural differences at 10 months or is predictive of later core symptoms of ASD.

\section{Final considerations on face and facial expression processing development}

In conclusion, what did this series of studies reveal about the general development of face and facial expression processing? We now know that at five months of age infants show cortical specialization for face (Chapter 3 and 4) but not for facial expression processing (Chapter 4). At 10 months of age the infant brain shows a similar sensitivity to faces as at five months (Chapter 3), and discriminates between emotional facial expressions based on the facial details (Chapter 5). These results are in line with previous developmental models on face and facial expression processing. It has been proposed that faces elicit specific activity in cortical regions from around two months after birth; before then, face processing is mainly subcortically-mediated (Johnson, 2001, 2005; Johnson et al., 1991; but see also Farroni et al., 2013). Facial expression processing abilities, on the other hand, are thought to emerge between five and seven months of age, when infants gain more experience with emotional facial expressions (Leppänen \& Nelson, 2009). Thus, the results of Chapter 3-5 nicely fit with these predictions, highlighting the differential developmental time course of face and facial expression processing.

The findings of the current thesis, however, do not inform about when and how the neural networks underlying face and facial expression processing emerge. Recall that Chapter 3 and 5 used ERPs, which do not allow for exact localization of brain activation. 
To fully understand the development of face and facial emotion processing networks the field needs to move to longitudinal designs that include measures of local brain activation (e.g. fNIRS). I believe that to capture brain specialization to faces, longitudinal studies should follow-up infants from shortly after birth until around 10 months of age. Starting to measure shortly after birth will also help disentangle when face processing switches from sub-cortical to cortical mechanisms. To track the emergence of facial expression processing abilities, longitudinal (fNIRS) studies between five and seven months would be appropriate according to relevant models (Leppänen \& Nelson, 2009).

\section{Conclusions}

I began this thesis with a brief glimpse of Laura, the prototypical 7-month-old. The overarching aim of this thesis was to aid the current understanding of how face and facial expression processing develop between the first and second semester of her first year of life. To this end, the first part of this dissertation focused on improving the definition of robust neural measures of infant face processing. In particular, Chapter 2 provides guidelines for developmental fNIRS researchers on how to process infant data and best deal with motion artifacts, which are a major source of noise in infant fNIRS studies. Chapter 3 identified the N290 and the P400 as robust measures of infant face processing between five and 10 months of age. The second part of this thesis investigated the development of face and facial expression processing between five and 10 months of age. My research suggests that face processing (i) recruits parts of the adult face-cortical network already at five months of age (Chapter 4), (ii) remains equally active between the first and second semester of life (Chapter 3). Turning to facial expression processing, this thesis further indicates that (iii) at five months infant brain responses do not discriminate between emotional facial expressions (Chapter 4); (iv) at 10 months infants mainly rely on facial details (HSF) to discriminate between emotional facial expressions; and (v) at this age infants with a higher likelihood to develop ASD do not discriminate between emotional facial expressions when either detailed (HSF) or global information (LSF) is displayed (Chapter 5). Combining the latter results with findings from previous 
research we suggest a developmental delay in the use of specific visual information to process facial expressions in ASD. Whereas this thesis contributes to the understanding of face and facial expression processing in five to 10-month-olds, the development of such skills across infancy is still not fully clear. To chart development we need large longitudinal studies combining different measures. 


\section{References}

Allison, T., Puce, A., \& McCarthy, G. (2000). Social perception from visual cues: role of the STS region. Trends in cognitive sciences, 4(7), 267-278. https://doi.org/10.1016/S13646613(00)01501-1

American Psychiatric Association. (2013). Diagnostic and statistical manual of mental disorders (5th ed.). Washington, D.C.: Author.

Behrendt, H. F., Firk, C., Nelson, C. A., \& Perdue, K. L. (2018). Motion correction for infant functional near-infrared spectroscopy with an application to live interaction data. Neurophotonics, 5(01), 1. https://doi.org/10.1117/1.NPh.5.1.015004

Blasi, A., Fox, S., Everdell, N., Volein, A., Tucker, L., Csibra, G., ... \& Elwell, C. (2007). Investigation of depth dependent changes in cerebral haemodynamics during face perception in infants. Physics in medicine and biology, 52(23), 6849. http://doi.org/10.1088/0031-9155/52/23/005

Bornstein, M. H., \& Arterberry, M. E. (2003). Recognition, discrimination and categorization of smiling by 5-month-old infants. Developmental Science, 6(5), 585-599. https://doi.org/10.1111/1467-7687.00314

Brigadoi, S., Ceccherini, L., Cutini, S., Scarpa, F., Scatturin, P., Selb, J., ... Cooper, R. J. (2014). Motion artifacts in functional near-infrared spectroscopy: A comparison of motion correction techniques applied to real cognitive data. NeuroImage, 85, 181-191. https://doi.org/10.1016/j.neuroimage.2013.04.082

Cooper, R., Selb, J., Gagnon, L., Phillip, D., Schytz, H. W., Iversen, H. K., ... \& Boas, D. A. (2012). A systematic comparison of motion artifact correction techniques for functional near-infrared spectroscopy. Frontiers in Neuroscience, 6, 147. https://doi.org/10.3389/fnins.2012.00147

D'Souza, H., \& Karmiloff-Smith, A. (2016). Neurodevelopmental disorders. Wiley Interdisciplinary Reviews: Cognitive Science, 8(1-2), e1398. http://doi.org/10.1002/wcs.1398

DeBoer, T., Scott, L. S., \& Nelson, C. A. (2007). Methods for acquiring and analyzing infant event-related potentials. In de Haan M. (Ed.), Infant EEG and event-related potentials, (pp. 5-37). New York, NY, US: Psychology Press.

de Haan, M, \& Nelson, C. A. (1999). Brain activity differentiates faces and object processing in 6-month-old infants. Developmental Psychology, 35(4), 1113-1121.

de Haan, M., Johnson, M. H., \& Halit, H. (2003). Development of face-sensitive event-related potentials during infancy: a review. International Journal of Psychophysiology, 51(1), 4558 . 
Estes, A., Zwaigenbaum, L., Gu, H., John, T. S., Paterson, S., Elison, J. T., ... \& IBIS network. (2015). Behavioral, cognitive, and adaptive development in infants with autism spectrum disorder in the first 2 years of life. Journal of neurodevelopmental disorders, 7(1), 24. http://doi.org/10.1186/s11689-015-9117-6

Farroni, T., Chiarelli, A. M., Lloyd-Fox, S., Massaccesi, S., Merla, A., Di Gangi, V., ... \& Johnson, M. H. (2013). Infant cortex responds to other humans from shortly after birth. Scientific reports, 3, 2851. https://doi.org/10.1038/srep02851

Fox, S. E., Wagner, J., Shrock, C. L., Flusberg, H. T., \& Nelson, C. A. (2013). Neural processing of facial identity and emotion in infants at high-risk for autism spectrum disorders. Frontiers in Human Neuroscience, 7, 89. https://doi.org/10.3389/fnhum.2013.00089

Gervain, J., Mehler, J., Werker, J. F., Nelson, C. A., Csibra, G., Lloyd-Fox, S., ... \& Aslin, R. N. (2011). Near-infrared spectroscopy: a report from the McDonnell infant methodology consortium. Developmental cognitive neuroscience, 1(1), 22-46. https://doi.org/10.1016/j.dcn.2010.07.004

Guy, M. W., Zieber, N., \& Richards, J. E. (2016). The Cortical Development of Specialized Face Processing in Infancy. Child Development, 87(5), 1581-1600. https://doi.org/10.1111/cdev.12543

Halit, H., Csibra, G., Volein, A., \& Johnson, M. H. (2004). Face-sensitive cortical processing in early infancy. Journal of Child Psychology and Psychiatry, 45(7), 1228-1234. http://doi.org/10.1111/j.1469-7610.2004.00321.x

Haxby, J., Hoffman, E., \& Gobbini, M. (2002). Human neural systems for face recognition and social communication. Biological psychiatry, 51(1), 59-67. http://doi.org/10.1016/S00063223(01)01330-0

Hoehl, S., Striano, T., 2010b. The development of emotional face and eye gaze processing. Developmental Sciences 13 (6), 813-825. http://doi.org/10.1111/j.14677687.2009.00944.x.

Issard, C., \& Gervain, J. (2018). Variability of the hemodynamic response in infants: Influence of experimental design and stimulus complexity. Developmental cognitive neuroscience, 33, 182-193. https://doi.org/10.1016/j.dcn.2018.01.009

Jessen, S., \& Grossmann, T. (2017). Exploring the role of spatial frequency information during neural emotion processing in human infants. Frontiers in human neuroscience, 11, 486. http://doi.org/10.3389/fnhum.2017.00486

Johnson, M. H. (2001). Functional brain development in humans. Nature Reviews Neuroscience, 2(7), 475. https://doi.org/10.1038/35081509

Johnson, M. H. (2005). Subcortical face processing. Nature Reviews Neuroscience, 6(10), 766774. https://doi.org/10.1038/nrn1766 
Johnson, M. H., Dziurawiec, S., Ellis, H., \& Morton, J. (1991). Newborns' preferential tracking of face-like stimuli and its subsequent decline. Cognition, 40(1-2), 1-19.

Johnson, M. H., Senju, A., \& Tomalski, P. (2015). The two-process theory of face processing: modifications based on two decades of data from infants and adults. Neuroscience \& Biobehavioral Reviews, 50, 169-179. https://doi.org/10.1016/j.neubiorev.2014.10.009

Jones, E. J., Venema, K., Earl, R., Lowy, R., Barnes, K., Estes, A., ... \& Webb, S. J. (2016). Reduced engagement with social stimuli in 6-month-old infants with later autism spectrum disorder: a longitudinal prospective study of infants at high familial risk. Journal of neurodevelopmental disorders, 8(1), 7. http://doi.org/10.1186/s11689016-9139-8

Kabdebon, C., Leroy, F., Simmonet, H., Perrot, M., Dubois, J., \& Dehaene-Lambertz, G. (2014). Anatomical correlations of the international 10-20 sensor placement system in infants. NeuroImage, 99, 342-356. https://doi.org/10.1016/j.neuroimage.2014.05.046

Karmiloff-Smith, A. (2015). An alternative to domain-general or domain-specific frameworks for theorizing about human evolution and ontogenesis. AIMS Neuroscience, 2(2), 91104. https://doi.org/10.3934/Neuroscience.2015.2.91

Leppänen, J. M., \& Nelson, C. A. (2009). Tuning the developing brain to social signals of emotions. Nature Reviews Neuroscience, 10(1), 37-47. https://doi.org/10.1038/nrn2554

Libertus, K., Landa, R. J., \& Haworth, J. L. (2017). Development of Attention to Faces during the First 3 Years: Influences of Stimulus Type. Frontiers in Psychology, 8, 1976.

Libertus, K., Sheperd, K. A., Ross, S. W., \& Landa, R. J. (2014). Limited fine motor and grasping skills in 6-month-old infants at high risk for autism. Child development, 85(6), 2218-2231. https://doi.org/10.1111/cdev.12262

Lloyd-Fox, S., Blasi, A., \& Elwell, C. E. (2010). Illuminating the developing brain: The past, present and future of functional near infrared spectroscopy. Neuroscience and Biobehavioral Reviews, 34(3), 269-284. http://doi.org/10.1016/j.neubiorev.2009.07.008

Lloyd-Fox, S., Blasi, A., Elwell, C., Charman, T., Murphy, D., \& Johnson, M. (2013). Reduced neural sensitivity to social stimuli in infants at risk for autism. Proceedings of the Royal Society B: Biological Sciences, 280(1758), 20123026-20123026. http://doi.org/10.1098/rspb.2012.3026

Lloyd-Fox, S., Richards, J. E., Blasi, A., Murphy, D. G. M., Elwell, C. E., \& Johnson, M. H. (2014). Coregistering functional near-infrared spectroscopy with underlying cortical areas in infants. Neurophotonics, 1(2), 025006. https://doi.org/10.1117/1.NPh.1.2.025006

Luck, S. J. (2014). An Introduction to the Event-Related Potential Technique. MIT Press.

Munsters, N.M., van Ravenswaaij, H., van den Boomen, C., \& Kemner, C. (2019). Test- retest reliability of infant event related potentials evoked by faces. Neuropsychologia, 126, 20 26. http://doi.org/ 10.1016/j.neuropsychologia.2017.03.030 
Nakato, E., Otsuka, Y., Kanazawa, S., Yamaguchi, M., \& Kakigi, R. (2011). Distinct differences in the pattern of hemodynamic response to happy and angry facial expressions in infants-A near-infrared spectroscopic study. NeuroImage, 54(2), 1600-1606. https://doi.org/10.1016/j.neuroimage.2010.09.021

Nakato, E., Otsuka, Y., Kanazawa, S., Yamaguchi, M., Watanabe, S., \& Kakigi, R. (2009). When do infants differentiate profile face from frontal face? A near-infrared spectroscopic study. Human Brain Mapping, 30(2), 462-472. http://doi.org/10.1002/hbm.20516

Nelson, C. A., \& McCleery, J. P. (2008). Use of event-related potentials in the study of typical and atypical development. Journal of the American Academy of Child \& Adolescent Psychiatry, 47(11), 1252-1261.

Otsuka, Y., Nakato, E., Kanazawa, S., Yamaguchi, M., Watanabe, S., \& Kakigi, R. (2007). Neural activation to upright and inverted faces in infants measured by near infrared spectroscopy. NeuroImage, 34(1), 399-406. http://doi.org/10.1016/j.neuroimage.2006.08.013

Ozonoff, S., Young, G. S., Carter, A., Messinger, D., Yirmiya, N., Zwaigenbaum, L., ... \& Hutman, T. (2011). Recurrence risk for autism spectrum disorders: a Baby Siblings Research Consortium study. Pediatrics, 128(3), e488-e495.

Peltola, M.J., Leppänen, J.M., Mäki, S., Hietanen, J.K., 2009. Emergence of enhanced attention to fearful faces between 5 and 7 months of age. Social Cognitive Affective Neuroscience, 4 (2), 134-142. http://dx.doi.org/10.1093/scan/nsn046.

Pinti, P., Scholkmann, F., Hamilton, A., Burgess, P., \& Tachtsidis, I. (2018). Current status and issues regarding pre-processing of fNIRS neuroimaging data: An investigation of diverse signal filtering methods within a General Linear Model framework. Frontiers in human neuroscience, 12. http://doi.org/10.3389/fnhum.2018.00505

Tzourio-Mazoyer, N., De Schonen, S., Crivello, F., Reutter, B., Aujard, Y., \& Mazoyer, B. (2002). Neural correlates of woman face processing by 2-month-old infants. NeuroImage, 15, 454-461. http://dx.doi.org/ 10.1006/nimg.2001.0979

van den Boomen, C., Munsters, N., \& Kemner, C. (2019). Emotion processing in the infant brain: The importance of local information. Neuropsychologia, 126, 62-68. https://doi.org/10.1016/j.neuropsychologia.2017.09.006

van den Boomen, C., van der Smagt, M. J., \& Kemner, C. (2012). Keep your eyes on development: the behavioral and neurophysiological development of visual mechanisms underlying form processing. Frontiers in Psychiatry, 3, 16.

Vlamings, P. H. J. M., Goffaux, V., \& Kemner, C. (2009). Is the early modulation of brain activity by fearful facial expressions primarily mediated by coarse low spatial frequency information? Journal of Vision, 9(5), 12.1-13. http://doi.org/10.1167/9.5.12 
Chapter 6

Vlamings, P. H. J. M., Jonkman, L. M., van Daalen, E., van der Gaag, R. J., \& Kemner, C. (2010). Basic abnormalities in visual processing affect face processing at an early age in autism spectrum disorder. Biological Psychiatry, 68(12), 1107-1113.

http://doi.org/10.1016/j.biopsych.2010.06.024

Webb, S. J., Jones, E. J., Merkle, K., Venema, K., Greenson, J., Murias, M., \& Dawson, G. (2011). Developmental change in the ERP responses to familiar faces in toddlers with autism spectrum disorders versus typical development. Child development, 82(6), 18681886. http://doi.org/10.1111/j.1467-8624.2011.01656.x

Webb, S. J., Neuhaus, E., \& Faja, S. (2017). Face perception and learning in autism spectrum disorders. The Quarterly Journal of Experimental Psychology, 70(5), 970-986. http://dx.doi.org/10.1080/17470218.2016.1151059

White, H., Chroust, A., Heck, A., Jubran, R., Galati, A., \& Bhatt, R. S. (2019). Categorical Perception of Facial Emotions in Infancy. Infancy, 24(2), 139-161. https://doi.org/10.1111/infa.12275 


Appendix I

Nederlandse Samenvatting 
Kinderen zijn net kleine onderzoekers, die de wereld ontdekken door alles wat hun aandacht trekt te bekijken en aan te raken. Neem Laura, een meisje van zeven maanden oud, dat op de grond met haar speelgoed aan het spelen is. Opeens wordt haar aandacht getrokken door het stopcontact aan de muur. Ze laat meteen haar speelgoed vallen en kruipt richting het stopcontact. Als er niks gebeurt zal Laura het stopcontact aanraken om het te onderzoeken. Echter, als haar moeder haar met een angstige blik aankijkt is de kans groot dat Laura stopt en het stopcontact niet aan zal raken. Dit voorbeeld illustreert dat het vermogen om gezichten te onderscheiden van andere objecten en vervolgens het herkennen en begrijpen van gezichtsuitdrukkingen cruciaal is voor succesvolle sociale communicatie tussen mensen, in het bijzonder voor pre-verbale kinderen zoals Laura.

Gedurende het eerste levensjaar vinden er belangrijke veranderingen plaats in de verwerking van gezichten en gezichtsuitdrukkingen. Kinderen tussen de zes en negen maanden oud kunnen bijvoorbeeld gezichten al categoriseren op kenmerken zoals geslacht, ras en aantrekkelijkheid. Vele studies richten zich op het blootleggen van de hersenactiviteit die onderliggend is aan het verwerken van gezichten en emotionele uitdrukkingen. Het is daarbij belangrijk om te ontdekken welke factoren van invloed kunnen zijn op de ontwikkeling van deze verwerkingsprocessen. Kennis over hoe normaal ontwikkelende baby's gezichten verwerken helpt ons om te begrijpen hoe de waarneming van gezichten en gezichtsuitdrukkingen verandert gedurende het eerste levensjaar en geeft meer inzicht in de processen die onderliggend zijn aan de sociale ontwikkeling van kinderen. Daarnaast kan deze kennis uiteindelijk leiden tot het beter begrijpen van stoornissen waarbij er problemen zijn in de sociale ontwikkeling, zoals bij een autisme spectrum stoornis (ASS).

Eerdere studies met volwassen proefpersonen laten zien dat er een aantal specifieke neurale netwerken betrokken zijn bij het verwerken van gezichten en gezichtsuitdrukkingen. Gezichten worden via twee paden in de hersenen verwerkt: een subcorticaal pad wat zorgt voor een snelle verwerking en een corticaal pad wat langzamer is, maar zorgt voor een meer gedetailleerde verwerking van gezichten. Delen 
van deze netwerken voor het verwerken van gezichten (e.g., de amygdala en de superior temporale sulcus) zijn ook betrokken bij het verwerken van gezichtsuitdrukkingen, naast andere corticale gebieden zoals de orbito-frontale cortex. Hoewel er veel bekend is over deze neurale netwerken in het volwassen brein, is er nog minder bekend over hoe deze netwerken zich ontwikkelen in het babybrein. Het doel van mijn proefschrift is dan ook om meer te weten te komen over de ontwikkeling van de verwerking van gezichten en emotionele gezichtsuitdrukking gedurende het eerste levensjaar.

Er zijn tot op heden twee verschillende meettechnieken die veelvuldig ingezet worden voor het meten van hersenactiviteit bij baby's; 'functional near-infrared spectroscopy' (fNIRS) en 'event-related potentials' (ERPs). Eerdere fNIRS en ERP studies geven al een indicatie van de ontwikkeling van de verwerking van gezichten en gezichtsuitdrukkingen gedurende het eerste levensjaar. Specifieke gezichtsverwerking lijkt in het eerste half jaar al aanwezig te zijn, waarbij deels dezelfde hersengebieden betrokken zijn als in het volwassen brein (e.g., Nakato et al., 2009). Het verwerken van gezichtsuitdrukkingen lijkt daarentegen wat later te ontwikkelen, zo rond de leeftijd van zes maanden (Leppänen \& Nelson, 2009). Op een leeftijd van zeven maanden gebruiken baby's hersengebieden voor het verwerken van gezichtsuitdrukkingen die ook onderdeel zijn van het emotionele verwerkingssysteem van volwassenen (Fox, Wagner, Shrock, Flusberg, \& Nelson, 2013; Nakato, Otsuka, Kanazawa, Yamaguchi, \& Kakigi, 2011). Hoewel deze studies suggereren dat de netwerken onderliggend aan het verwerken van gezichten en gezichtsuitdrukkingen functioneel zijn in het eerste levensjaar, is er nog wel inconsistentie in de literatuur over wanneer en hoe deze verwerkingsprocessen zich ontwikkelen in de babytijd. Ook weten we weinig over mogelijke individuele verschillen tussen kinderen in gezichts- en emotieverwerking. Het is belangrijk om deze verschillen te identificeren om een goed beeld de krijgen van de spreiding in typische ontwikkeling en de grens met een atypische ontwikkeling van deze verwerkingsprocessen.

In dit proefschrift zullen verschillende vragen met betrekking tot de ontwikkeling van het verwerken van gezichten en gezichtsuitdrukkingen beantwoord worden met behulp van fNIRS en ERPs: (i) Welke corticale gebieden zijn betrokken bij 
gezichtsverwerking in 5 maanden oude baby's (Hoofdstuk 4)?; (ii) Vindt er ontwikkeling in gezichtsverwerking plaats tussen de leeftijd van 5 en 10 maanden (Hoofdstuk 3)?; (iii) Maakt het babybrein onderscheid tussen verschillende gezichtsuitdrukkingen op een leeftijd van 5 maanden en welke corticale gebieden zijn daarbij betrokken (Hoofdstuk 4); en (iv) zijn een verhoogde kans op het ontwikkelen van ASS en de manier waarop visuele informatie verwerkt wordt (i.e., details vs. globale informatie) factoren die invloed kunnen hebben op individuele verschillen in de verwerking van gezichtsuitdrukkingen bij 10 maanden oude baby's (Hoofdstuk 5)?

Daarnaast is een tweede doel van dit proefschrift om de gebruikte onderzoeksmethodes te verbeteren. Hoewel fNIRS en ERPs veelvuldig gebruikt worden in babyonderzoek, is er nog geen consensus over wat de meest robuuste maten zijn om gezichtsverwerking bij baby's te onderzoeken. Het identificeren van de meest robuuste maten is essentieel voor het interpreteren van data en het maken van vergelijkingen tussen studies. Daarom worden in dit proefschrift nog twee methodologische vragen beantwoord: (i) Wat is de optimale aanpak voor de correctie van bewegingsartefacten in baby fNIRS data (Hoofdstuk 2)?; en (ii) welke van de in de literatuur gerapporteerde ERP componenten zijn robuuste maten voor gezichtsverwerking in baby's (Hoofdstuk 3)?

In het volgende deel van deze samenvatting zal ik de genoemde vragen bespreken, resultaten van de studies uiteenzetten en de bevindingen bediscussiëren. In het eerste deel zal ik focussen op de methodologische vragen, waarna in deel twee de vragen wat betreft de ontwikkeling van het verwerken van gezichten en gezichtsuitdrukkingen besproken worden. Ook zal ik aandacht geven aan potentiële limitaties van mijn studies en geef ik suggesties voor toekomstig onderzoek. 


\section{Deel 1 - Methode}

\section{Hoe komen we tot robuuste maten voor gezichtsverwerking in baby's?}

\section{fNIRS: Wat is de optimale aanpak voor de correctie van bewegingsartefacten in baby}

\section{fNIRS data?}

fNIRS is een neuro-imagingtechniek die hersenactivatie afleidt door het kwantificeren van veranderingen in de hoeveelheid oxy-hemoglobine $(\mathrm{HbO})$ en deoxy-hemoglobine $(\mathrm{HbR})$ in het bloed. Deze concentraties $\mathrm{HbO}$ en $\mathrm{HbR}$ worden bepaald op basis van de verandering in de intensiteit van bijna-infrarood licht wat uitgestuurd wordt door een lichtbron en weer opgevangen wordt door een detector, welke op de hoofdhuid geplaatst worden (Obrig \& Villringer, 2003). Wanneer een hersengebied geactiveerd wordt door een stimulus zal dit gebied een toename van $\mathrm{HbO}$ en een afname van $\mathrm{HbR}$ laten zien, wat ook wel de hemodynamische respons functie (HRF) genoemd wordt. fNIRS wordt veel gebruikt in babyonderzoek omdat het een non-invasieve techniek is die makkelijk gebruikt kan worden bij niet-slapende baby's. Er zijn echter ook enkele nadelen verbonden aan deze techniek. Het grootste struikelpunt is hoe om te gaan met bewegingsartefacten in de data. Als hier niet goed mee omgegaan wordt kan dit gevolgen hebben voor de gemiddelde HRF en kan men hersenactivatie waarnemen in gebieden die eigenlijk niet actief waren of juist echte hersenactivatie maskeren. Tot op heden is er nog maar één studie die de effecten van een veelgebruikte correctie voor bewegingsartefacten in baby fNIRS data onderzocht heeft (Behrendt, Firk, Nelson, \& Perdue, 2018). Hoofdstuk 2 uit dit proefschrift is een toevoeging op dit artikel door het maken van een vergelijking tussen verschillende veelgebruikte correctiemethodes en het combineren van verschillende methodes. De effecten van de correctiemethodes Spline, Wavelet en Spline + Wavelet (Brigadoi et al., 2014; Cooper et al., 2012) zijn uitgetest op gesimuleerde data en drie verschillende datasets met baby fNIRS data. Omdat babydata veel ruis bevat was de verwachting dat de combinatie van twee verschillende correctiemethodes zou leiden tot betere resultaten.

Als eerste laten de resultaten van Hoofdstuk 2 zien dat het verwijderen van alle trials waarin een bewegingsartefact aanwezig is het meest negatieve resultaat oplevert in 
het zowel het herstellen van de HRF in de gesimuleerde data als in het reduceren van de variabiliteit in HRFs binnen en tussen proefpersonen voor de datasets met echte data (waarbij de assumptie is dat hogere variabiliteit vooral toegeschreven kan worden aan de aanwezigheid van bewegingsartefacten). Door het gebruik van deze methode bleef ook minder dan 50\% van alle trials beschikbaar voor verdere analyses. Voor het verkrijgen van betrouwbare maten voor hersenactivatie is het essentieel dat zoveel mogelijk trials meegenomen kunnen worden in de uiteindelijke analyses. Hoewel het verwijderen van trials met een bewegingsartefact nog vaak gebeurt in baby fNIRS onderzoek, laat de studie in Hoofdstuk 2 dus zien dat het gunstiger is voor de datakwaliteit om een correctie uit te voeren dan om trials met een bewegingsgartefact simpelweg te verwijderen.

Ten tweede is in Hoofdstuk 2 gevonden dat het combineren van twee correctiemethodes (Spline + Wavelet) het meest effectief is in (i) het regenereren van de HRF in data met lage en hoge percentages bewegingsartefacten; (ii) het reduceren van de variabiliteit in HRFs binnen en tussen proefpersonen; en (iii) het behouden van bijna alle trials met een bewegingsartefact voor verdere analyses. We adviseren voor toekomstige studies dan ook om deze twee correctiemethodes te combineren, zeker als er veel bewegingsartefacten aanwezig zijn in de data. Zijn er weinig bewegingsartefacten aanwezig, dan volstaat alleen het toepassen van Wavelet ook voor het corrigeren van de data. Deze resultaten dragen bij aan de standaardisatie van de verwerking van baby fNIRS data en hebben hopelijk als resultaat dat de uitkomsten van studies betrouwbaarder en beter vergelijkbaar worden. 
ERPs: Welke van de in de literatuur gerapporteerde ERP-componenten zijn robuuste maten voor gezichtsverwerking gedurende het eerste levensjaar?

ERPs zijn veranderingen in elektrische activiteit na het tonen van een stimulus, gemeten met elektroden op de hoofdhuid. ERPs worden veelvuldig gebruikt in onderzoek naar gezichtsverwerking, zowel bij volwassenen als bij baby's. Hierbij wordt gekeken naar het verschil in elektrische hersenactiviteit voor stimuli van gezichten en andere objecten. Eerder onderzoek heeft drie ERP-componenten geïdentificeerd die betrokken zijn bij gezichtsverwerking bij baby's: de N290, de P400 en de 'Negative central' (Nc). Er wordt gedacht dat deze drie componenten verschillende stadia van gezichtsverwerking reflecteren. De N290 en de P400 zijn gelinkt aan de vroege stadia van gezichtsverwerking en zijn mogelijk ook de voorlopers van de N170 component, welke zeer belangrijk is in de gezichtsverwerking bij volwassen (de Haan, Johnson, \& Halit, 2003). De Nc is niet specifiek gerelateerd aan het verwerken van gezichten, maar lijkt meer indicatief te zijn voor de mate van aandacht en mogelijke voorkeuren in kijkgedrag (e.g., Courchesne, Ganz, \& Norcia, 1981; de Haan \& Nelson, 1997; de Haan et al., 2003). Hoewel vele studies onderzoek gedaan hebben naar de ERPs die betrokken lijken te zijn bij gezichtsverwerking, is er nog steeds verdeeldheid over welke component het proces van gezichtsverwerking gedurende de babytijd het beste weergeeft. Eerder babyonderzoek laat inderdaad tegenstrijdige resultaten zien als het gaat om hoe goed de eerdergenoemde componenten een onderscheid maken tussen stimuli van gezichten en andere objecten (cf. Table 3.1). Er zijn verschillende verklaringen voor deze tegenstrijdige resultaten. Ten eerste hebben eerdere onderzoeken maar kleine aantallen proefpersonen, waardoor effectgroottes uitvergroot worden en het lastig is om resultaten te repliceren (Frank et al., 2017). Ten tweede wordt de gezichtsverwerking vaak maar op één bepaalde leeftijd gemeten, of wordt er gebruik gemaakt van een crosssectioneel design in plaats van een longitudinaal design, wat het effect van ontwikkeling kan overschaduwen (maar zie Webb, Long, \& Nelson, 2005). Ten derde rapporteren studies vaak maar een subset van mogelijke ERP-componenten betrokken bij gezichtsherkenning, wat vergelijkingen tussen studies onmogelijk maakt. Om te 
voorkomen dat deze punten invloed hebben op de resultaten, is in Hoofdstuk 3 een grote longitudinale dataset $(\mathrm{N}=80)$ gebruikt te testen welke van de in de literatuur gerapporteerde ERP-componenten (i.e., N290, P400, Nc) robuuste maten zijn om te kijken naar gezichtsverwerking in de babytijd. Een robuuste maat zou in een grote groep van de 5 maanden oude baby's betrokkenheid bij de verwerking van gezichten moeten laten zien, en ook 10 maanden oude baby's zouden dit patroon moeten laten zien.

De analyses van Hoofdstuk 3 laten zien dat alle genoemde ERP-componenten (i.e., N290, P400, Nc) een verschil in activatie laten zien voor stimuli van gezichten vergeleken met stimuli van huizen in zowel 5 maanden als 10 maanden oude baby's. De amplitudes voor de N290 en de P400 waren negatiever voor gezichten dan voor huizen, terwijl het tegenovergestelde patroon gevonden is voor de $\mathrm{Nc}$ (= dominante verwerkingspatroon). Gebaseerd op deze analyses op groepsniveau leidt tot de conclusie dat alle drie de ERP-componenten robuuste maten zijn voor gezichtsverwerking gedurende het eerste levensjaar. Als we naar individuele proefpersonen kijken zien we echter een ander patroon. Markov-modellen tonen aan dat de N290 en de P400 robuustere maten zijn dan de $\mathrm{Nc}$, omdat voor deze componenten (i) een grotere proportie van de 5 maanden oude baby's het dominante verwerkingspatroon laten zien $(\mathrm{N} 290 / \mathrm{P} 400>83 \%, \mathrm{Nc}=60 \%)$; (ii) een grotere proportie van deze baby's ook met 10 maanden dit dominante verwerkingspatroon nog laat zien $(\mathrm{N} 290 / \mathrm{P} 400>85 \%$, Nc $=$ $67 \%$ ); en (iii) de meeste kinderen met een niet-dominant verwerkingspatroon op een leeftijd van 5 maanden alsnog switchen naar het dominante verwerkingspatroon met 10 maanden $(\mathrm{N} 290 / \mathrm{P} 400=67-93 \%, \mathrm{Nc}=53 \%)$. Het patroon beschreven onder punt (iii) kan het gevolg zijn van een meetfout, of van een ontwikkelingsachterstand waarbij baby's pas na de leeftijd van 5 maanden in staat zijn om gezichten goed te verwerken. Concluderend laat Hoofdstuk 3 zien dat de N290 en de P400 de meest robuuste maten zijn voor het meten van gezichtsverwerking gedurende het eerste levensjaar. Deze resultaten dragen bij aan de kennis over de rol van de genoemde ERPs in het proces van gezichtsverwerking, wat belangrijk is voor het onderzoek naar de typische en atypische ontwikkeling van dit proces. 


\section{Deel 2 - Ontwikkeling}

\section{Hoe verandert de verwerking van gezichten en gezichtsuitdrukkingen tussen de leeftijd van 5 en 10 maanden oud?}

In dit deel van de samenvatting bespreek ik de resultaten wat betreft de ontwikkeling van de verwerking van gezichten en gezichtsuitdrukkingen gedurende het eerste levensjaar, zoals deze besproken zijn in Hoofdstuk 3, 4, en 5. Ik zal eerste focussen op het verwerken van gezichten in het algemeen (Hoofdstuk 3 en 4). Daarna zullen de resultaten wat betreft de verwerking van gezichtsuitdrukkingen (Hoofdstuk 4 en 5) besproken worden.

\section{Het verwerken van gezichten}

In hoofdstuk 4 heb ik met behulp van fNIRS onderzocht welke corticale hersengebieden betrokken zijn bij het verwerken van gezichten in 5 maanden oude baby's. Ik heb specifiek gekeken naar de HRF in reactie op foto's van gezichten, foto's van huizen en een baseline stimulus in drie verschillende corticale gebieden in de rechterhemisfeer: occipitale, temporale en frontale gebieden. Gebaseerd op eerder fNIRS onderzoek met dezelfde leeftijdsgroep (Blasi et al., 2007; Nakato et al., 2009) was de verwachting dat er verhoogde hersenactivatie in reactie op gezichten te zien zou zijn in temporale en/of occipitale gebieden.

De resultaten van Hoofdstuk 4 laten inderdaad een toename van $\mathrm{HbO}$ zien in occipitale gebieden in reactie op foto's van gezichten. Deze toename was niet aanwezig in de temporale gebieden. Deze resultaten suggereren dat het proces van gezichtsherkenning, in ieder geval gedeeltelijk, aanwezig en functioneel is in het brein van 5 maanden oude baby's. Het vinden van verhoogde hersenactivatie in reactie op gezichten in occipitale gebieden komt overeen met eerdere fNIRS studies die een soortgelijke activatie vonden in 4 maanden oude (Blasi et al., 2007) en 5 maanden oude baby's (Nakato et al., 2009). We verwachten dat deze activatie afkomstig is van de 'occipital face area' (OFA) welke ook onderdeel is van het netwerk voor 
gezichtsverwerking in volwassenen (Haxby et al., 2002). Het volwassen netwerk is dus al gedeeltelijk actief op een leeftijd van 5 maanden.

De resultaten van Hoofdstuk 3 wijzen er ook op dat 5 maanden oude baby's al een apart neuraal systeem hebben voor het verwerken van gezichten. Deze ERP-studie laat zijn dat de amplitudes voor de ERP-componenten gerelateerd aan gezichtsverwerking (i.e., N290, P400 en Nc) verschillend waren voor stimuli van huizen en stimuli van gezichten. In deze studie is ook getest of er verschillen zijn in het verwerken van gezichten tussen 5 en 10 maanden oude baby's. De resultaten laten zien dat deze twee leeftijdsgroepen soortgelijke amplitudes laten zien voor de eerdergenoemde ERPcomponenten in reactie op gezichten en huizen. Dit suggereert dat er geen verdere ontwikkeling plaatsvindt in het verwerken van gezichten in de tussenliggende periode.

Het is echter van belang om te vermelden dat soortgelijke ERPs in 5 en 10 maanden oude baby's niet per sé betekent dat ze ook dezelfde neurale netwerken gebruiken om gezichten te verwerken. Omdat ERPs een lage spatiale resolutie hebben, is het moeilijk om te bepalen waar de opgevangen activatie precies vandaan komt (Luck, 2014). In vergelijking met ERPs heeft fNIRS een hogere spatiale resolutie. Toekomstig onderzoek zou daarom beide technieken moeten combineren in longitudinale studies, zodat te controleren is of de gemeten ERPs een betrouwbare reflectie zijn voor de conclusie dat de onderliggende neurale netwerken zich ook niet verder ontwikkelen van een leeftijd van 5 naar 10 maanden oud. De combinatie van verschillende technieken zorgt voor sterkere en beter geïnformeerde conclusies, wat leidt tot meer inzicht in de normale ontwikkeling van gezichtsverwerking.

\section{Toekomstig onderzoek-Het verwerken van gezichten}

In Hoofdstuk 3 en 4 is gezichtsverwerking pas getest vanaf een leeftijd van 5 maanden. Er zijn echter theoretische modellen die suggereren dat er vanaf een leeftijd van 2 maanden al speciale corticale neurale netwerken voor het verwerken van gezichten actief zijn in het brein (Johnson, 2001; 2005; Johnson, Dziurawiec, Ellis, \& Morton, 1991). Tot op heden zijn er geen fNIRS studies uitgevoerd met baby's jonger dan 4 maanden oud (maar zie Farroni et al., 2013 voor een fNIRS studie met pasgeborenen). Ook ERP- 
studies hebben niet gekeken naar gezichtsverwerking in kinderen jonger dan 3 maanden oud (Halit, Csibra, Volein, \& Johnson, 2004). Het zou interessant zijn om te bekijken, met zowel fNIRS als ERPs, op welke leeftijd het corticale neurale netwerk onderliggend aan gezichtsverwerking actief wordt en op welk moment in de ontwikkeling er in de ERP-componenten een onderscheid te zien is in het verwerken van gezichten en andere objecten.

Daarnaast is het interessant om te kijken wat individuele verschillen in ontwikkeling veroorzaakt. De resultaten van Hoofdstuk 3 toonden aan dat de meeste kinderen op een leeftijd van zowel 5 als 10 maanden een verschil in amplitude lieten zien tussen gezichten en huizen op alle drie de onderzochte ERP-componenten. Er zijn echter ook kinderen die pas bij 10 maanden een verschil in amplitudes laten zien. Dit zou kunnen betekenen dat sommige kinderen meer ervaring nodig hebben in het zien van gezichten voordat de processen onderliggend aan het verwerken van gezichten optimaal ontwikkeld zijn. Deze kinderen hebben dan een ontwikkelingsachterstand ten opzichte van hun leeftijdsgenoten. Deze kleine verschillen in ontwikkeling op jonge leeftijd kunnen grotere gevolgen hebben als kinderen opgroeien (Karmiloff-Smith, 2015). Een achterstand in gezichtsverwerking op een leeftijd van 5 maanden kan bijvoorbeeld merkbare gevolgen hebben voor de verdere sociale ontwikkeling en communicatievaardigheden op latere leeftijd. Toekomstig onderzoek zou deze vaardigheden op latere leeftijd kunnen testen om zo te kijken of een vroege achterstand in gezichtsverwerking inderdaad gevolgen heeft voor de verdere sociale ontwikkeling.

\section{Het verwerken van gezichtsuitdrukkingen}

In Hoofdstuk 4 heb ik getest of er op een leeftijd van 5 maanden al een speciaal corticaal netwerk aanwezig is in het babybrein voor het verwerken van gezichtsuitdrukkingen. We verwachtten activiteit te zien in temporale en frontale hersengebieden, omdat eerder fNIRS onderzoek betrokkenheid van deze gebieden aangetoond heeft in oudere baby's (e.g. Fox et al., 2013; Nakato et al., 2009). Onze resultaten toonden echter aan dat het netwerk betrokken bij het verwerken van gezichtsuitdrukkingen nog niet volledig ontwikkeld is op een leeftijd van 5 maanden. Activiteit in occipitale en frontale 
hersengebieden maakten] geen onderscheid tussen verschillende emoties. In de temporale gebieden is wel een klein verschil in activiteit waarneembaar. Deze gebieden lieten een significante afname in $\mathrm{HbO}$ zien bij het tonen van blije gezichten, terwijl er juist een toename in $\mathrm{HbO}$ te zien was voor bange gezichten, hoewel dit laatste effect net niet significant was.

De effecten die te zien zijn in de temporale gebieden zijn niet geheel zoals verwacht. Normaal zou activatie samengaan met een toename in $\mathrm{HbO}$, niet in een afname zoals te zien in reactie op de blije gezichten. Wat echter het meest interessant is, is dat we een merkbaar verschil zien in deze gebieden in het verwerken van blijde en bange gezichten. Er kan dus voorzichtig geconcludeerd worden dat 5 maanden oude baby's net beginnen met het maken van onderscheid tussen verschillende gezichtsuitdrukkingen. Dit is in overeenkomst met eerder onderzoek dat laat zien dat het netwerk wat emoties verwerkt ontwikkelt tussen de 5 en 7 maanden (Hoehl \& Striano, 2010; Leppänen \& Nelson, 2009; Peltola, Leppänen, Mäki, \& Hietanen, 2009). Het is mogelijk dat dit netwerk eerste een bepaalde ervaring nodig heeft met het waarnemen van verschillende gezichtsuitdrukkingen voordat het ook echt actief wordt en zich gaat specialiseren. Onze studie lijk het begin van deze specialisatie aan te tonen, maar meer longitudinale studies met oudere kinderen zijn nodig om de complete ontwikkeling van dit netwerk bloot te leggen.

Het onderscheiden van emoties is veelvuldig aangetoond in ERP-studies met kinderen ouder dan 7 maanden (voor een review, zie van den Boomen, Munsters, \& Kemner, 2019). Dit suggereert dat rond deze leeftijd het neurale netwerk onderliggend aan het verwerken van gezichtsuitdrukkingen in een vergevorderd stadium van ontwikkeling is (Leppänen \& Nelson, 2009). Om te kijken naar mogelijke afwijkingen in de ontwikkeling van dit netwerk in kinderen met een verhoogd risico op het ontwikkelen van ASS (i.e., deze kinderen hebben een oudere broer of zus met ASS) heb ik daarom gekeken naar kinderen van 10 maanden oud, die normaal gesproken al goed in staat moeten zijn om verschillende emoties te onderscheiden. Ik heb specifiek 
gekeken of de kinderen met een verhoogd risico andere visuele informatie gebruiken (i.e., globaal vs. gedetailleerd) om gezichtsuitdrukkingen te verwerken.

De resultaten laten zien dat 10 maanden oude baby's met een laag risico op ASS een verschil in amplitude op de P100, een ERP-component gerelateerd aan emotieverwerking, laten zien voor verschillende emoties, maar alleen als er gedetailleerde visuele informatie getoond wordt. Hieruit kan geconcludeerd worden dat normaal ontwikkelende 10 maanden oude baby's in staat zijn om onderscheid te maken tussen verschillende gezichtsuitdrukkingen, en dat ze hiervoor gebruik maken van details in gezichten en minder van de globale visuele informatie. Dit komt overeen met eerder onderzoek dat soortgelijke effecten vond in latere ERP-componenten zoals de N290 en de P400 (Jessen \& Grossman, 2017; van den Boomen et al., 2019). Mijn onderzoek voegt hieraan toe dat het effect van het type visuele informatie op het verwerken van emoties al aanwezig is in een nog eerder stadium van het visuele verwerkingsproces.

In tegenstelling tot de kinderen met een laag risico, laten kinderen met een verhoogd risico op ASS geen verschil in ERPs zien voor verschillende gezichtsuitdrukkingen, onafhankelijk van het type visuele informatie die getoond wordt. Deze kinderen lopen mogelijk achter in de ontwikkeling van de emotionele verwerkingsprocessen. De laag-risico kinderen in ons onderzoek gebruiken gedetailleerde visuele informatie voor het verwerken van emotionele informatie, kinderen met een verhoogd risico op autisme lijken deze informatie pas rond een leeftijd van 3 jaar te kunnen gebruiken (Vlamings et al., 2010). Dit is mogelijk een consequentie van het gegeven dat kinderen met een verhoogd risico op ASS ook achterlopen in het verwerken van gezichten op zich (Jones, 2016; Webb et al., 2011). Deze achterstanden kunnen vervolgens leiden tot moeilijkheden met het interpreteren van sociale informatie, wat kan leiden tot de symptomen kenmerkend voor mensen met ASS.

\section{Toekomstig onderzoek- Het verwerken van gezichtsuitdrukkingen}

In Hoofdstuk 4 is aangetoond dat 5 maanden oude kinderen geen verschil in hersenactivatie laten zien voor verschillende emoties. Gedragsonderzoek met deze 
leeftijdsgroep laat echter zien dat deze kinderen blije gezichten kunnen categoriseren (Bornstein en Arterberry, 2003) en dat ze onderscheid kunne maken tussen positieve en negatieve emoties (White et al., 2019). Hoe kan het dat gedragsonderzoek en neuroimaging onderzoek tot zulke verschillende conclusies leidt? Het is bijvoorbeeld mogelijk dat 5 maanden oude baby's onderscheid kunnen maken tussen verschillende emoties, maar dat dit ondersteund wordt door lagergelegen hersengebieden (e.g., fusiform gyrus en de amygdala) die niet meetbaar zijn met fNIRS. Een andere mogelijkheid is dat het babybrein nog niet in staat is om het onderscheid te maken tussen blije en bange gezichten, het contrast wat gebruikt is in de huidige studie, maar wel tussen andere emoties. Het is waarschijnlijk te simpel om te verwachten dat het babybrein opeens in staat is om alle verschillende emoties van elkaar te onderscheiden. Het is aannemelijker dat deze vaardigheid zich geleidelijk ontwikkelt. Om de exacte ontwikkeling van het verwerken van gezichtsuitdrukkingen te volgen zijn longitudinale studies nodig gedurende de tweede helft van het eerste levensjaar, waarbij emotieverwerking met zowel neuro-imaging als gedragsmaten getest wordt, waarbij meerdere emoties getoond worden.

Als we kijken naar Hoofdstuk 5 is het belangrijk om te vermelden dat dit onderzoek is uitgevoerd met kinderen met een verhoogd risico op ASS, maar er zijn geen officiële diagnoses beschikbaar voor deze kinderen. We kunnen dus ook niet stellen dat de gevonden resultaten gezien moeten worden als een vroege marker voor het ontwikkelen van ASS, omdat waarschijnlijk maar ongeveer $20 \%$ van de kinderen in de hoog-risico groep ook daadwerkelijk ASS zal ontwikkelen (cf., Ozonoff et al., 2011). Toekomstig onderzoek zal uit moeten wijzen of vooral de kinderen die ook daadwerkelijk gediagnosticeerd worden met ASS het meest afwijkende verwerkingspatroon lieten zien op een leeftijd van 10 maanden.

Daarnaast is het belangrijk om in gedachten te houden dat het manipuleren van afbeeldingen, zodat ze alleen globale of gedetailleerde informatie bevatten, ook gevolgen heeft voor de helderheid van de afbeelding. Deze veranderingen in helderheid lijken geen invloed te hebben op het verwerken van gezichtsuitdrukkingen in volwassenen 
(Vlamings, Goffaux, \& Kemner, 2009). Het is echter niet bekend of de verwerking van emoties in baby's wel beïnvloedt kan worden door deze veranderingen in helderheid. De gevoeligheid voor contrasten en het type visuele informatie verandert namelijk wel tijdens de ontwikkeling (van den Boomen et al., 2012). Het zou daarom interessant zijn om te zien of de resultaten van Hoofdstuk 5 nog steeds gevonden worden als de stimuli gecontroleerd worden op helderheid en andere visuele eigenschappen die mogelijk effect kunnen hebben op de visuele verwerking van de stimuli.

\section{Algemene conclusie}

Het overkoepelende doel van dit proefschrift was om meer kennis te vergaren over de ontwikkeling van het verwerken van gezichten en gezichtsuitdrukkingen gedurende het eerste levensjaar. In het eerste deel van dit proefschrift is gefocust op het verbeteren van de methodes gebruikt in babyonderzoek, om uit te zoeken wat de meest robuuste maten zijn voor hersenactivatie tijdens het verwerken van gezichten. Hoofdstuk 2 geeft advies over hoe de datakwaliteit van baby fNIRS data geoptimaliseerd kan worden en wat de juiste manier is om om te gaan met bewegingsartefacten in de data. Hoofdstuk 3 heeft laten zien dat de ERP-componenten N290 en P400 de meest robuuste maten zijn voor gezichtsverwerking in zowel 5 als 10 maanden oude baby's. In het tweede deel van dit proefschrift is gekeken naar de ontwikkeling van het verwerken van gezichten en gezichtsuitdrukkingen tussen een leeftijd van 5 en 10 maanden. Mijn onderzoek laat zijn dat gezichtsverwerking (i) al op 5 maanden oude leeftijd hersengebieden activeert die ook onderdeel zijn van het neurale netwerk voor gezichtsverwerking in volwassenen (Hoofdstuk 4); en (ii) dat er geen ontwikkeling in gezichtsverwerking plaatsvindt tussen een leeftijd van 5 en 10 maanden (Hoofdstuk 3). Als we kijken naar het verwerken van gezichtsuitdrukkingen, dan heb ik aangetoond dat (iii) op een leeftijd van 5 maanden het babybrein nog geen onderscheid maakt tussen verschillende gezichtsuitdrukkingen (Hoofdstuk 4); dat (iv) 10 maanden oude baby's details in gezichten gebruiken om emoties te herkennen; en (v) dat 10 maanden oude baby's met een verhoogd risico op autisme op deze leeftijd nog geen onderscheid maken tussen emoties, onafhankelijk van 


\section{Appendix I}

de visuele informatie die getoond wordt. Dit suggereert dat kinderen met een verhoogd risico op autisme achterlopen in hun ontwikkeling wat betreft het gebruiken van bepaalde visuele informatie voor het verwerken van gezichten. De resultaten van dit proefschrift geven al een goede inkijk in de ontwikkeling van de verwerking van gezichten en gezichtsuitdrukkingen. Echter, om echt een goed idee te krijgen van de veranderingen die plaatsvinden zijn longitudinale studies nodig met een nog jongere leeftijd als startpunt en meerdere meetmomenten gedurende het eerste levensjaar waarbij verwerkingsprocessen met meerdere methodes onderzocht worden. 


\section{Referenties}

Blasi, A., Fox, S., Everdell, N., Volein, A., Tucker, L., Csibra, G., ... \& Elwell, C. (2007). Investigation of depth dependent changes in cerebral haemodynamics during face perception in infants. Physics in medicine and biology, 52(23), 6849. http://doi.org/10.1088/0031-9155/52/23/005

Bornstein, M. H., \& Arterberry, M. E. (2003). Recognition, discrimination and categorization of smiling by 5-month-old infants. Developmental Science, 6(5), 585-599. https://doi.org/10.1111/1467-7687.00314

Brigadoi, S., Ceccherini, L., Cutini, S., Scarpa, F., Scatturin, P., Selb, J., ... Cooper, R. J. (2014). Motion artifacts in functional near-infrared spectroscopy: A comparison of motion correction techniques applied to real cognitive data. NeuroImage, 85, 181-191. https://doi.org/10.1016/j.neuroimage.2013.04.082

Cooper, R., Selb, J., Gagnon, L., Phillip, D., Schytz, H. W., Iversen, H. K., ... \& Boas, D. A. (2012). A systematic comparison of motion artifact correction techniques for functional near-infrared spectroscopy. Frontiers in Neuroscience, 6, 147. https://doi.org/10.3389/fnins.2012.00147

Courchesne, E., Ganz, L., \& Norcia, A. M. (1981). Event-related brain potentials to human faces in infants. Child development, 804-811. https://doi.org/10.2307/1129080

de Haan, M, \& Nelson, C. A. (1999). Brain activity differentiates faces and object processing in 6-month-old infants. Developmental Psychology, 35(4), 1113-1121.

de Haan, M., Johnson, M. H., \& Halit, H. (2003). Development of face-sensitive event-related potentials during infancy: a review. International Journal of Psychophysiology, 51(1), 4558.

Farroni, T., Chiarelli, A. M., Lloyd-Fox, S., Massaccesi, S., Merla, A., Di Gangi, V., ... \& Johnson, M. H. (2013). Infant cortex responds to other humans from shortly after birth. Scientific reports, 3, 2851. https://doi.org/10.1038/srep02851

Fox, S. E., Wagner, J., Shrock, C. L., Flusberg, H. T., \& Nelson, C. A. (2013). Neural processing of facial identity and emotion in infants at high-risk for autism spectrum disorders. Frontiers in Human Neuroscience, 7, 89. https://doi.org/10.3389/fnhum.2013.00089

Frank, M. C., Bergelson, E., Bergmann, C., Cristia, A., Floccia, C., Gervain, J., ... \& LewWilliams, C. (2017). A collaborative approach to infant research: Promoting reproducibility, best practices, and theory-building. Infancy, 22(4), 421-435. https://doi.org/10.1111/infa.12182

Halit, H., Csibra, G., Volein, A., \& Johnson, M. H. (2004). Face-sensitive cortical processing in early infancy. Journal of Child Psychology and Psychiatry, 45(7), 1228-1234.

http://doi.org/10.1111/j.1469-7610.2004.00321.x 
Haxby, J., Hoffman, E., \& Gobbini, M. (2002). Human neural systems for face recognition and social communication. Biological psychiatry, 51(1), 59-67. http://doi.org/10.1016/S00063223(01)01330-0

Hoehl, S., Striano, T., 2010. The development of emotional face and eye gaze processing. Dev. Sci. 13 (6), 813-825. http://doi.org/10.1111/j.1467-7687.2009.00944.x.

Jessen, S., \& Grossmann, T. (2017). Exploring the role of spatial frequency information during neural emotion processing in human infants. Frontiers in human neuroscience, 11, 486. http://doi.org/10.3389/fnhum.2017.00486

Johnson, M. H. (2005). Subcortical face processing. Nature Reviews Neuroscience, 6(10), 766774. https://doi.org/10.1038/nrn1766

Johnson, M. H., Dziurawiec, S., Ellis, H., \& Morton, J. (1991). Newborns' preferential tracking of face-like stimuli and its subsequent decline. Cognition, 40(1-2), 1-19.

Jones, E. J., Venema, K., Earl, R., Lowy, R., Barnes, K., Estes, A., ... \& Webb, S. J. (2016). Reduced engagement with social stimuli in 6-month-old infants with later autism spectrum disorder: a longitudinal prospective study of infants at high familial risk. Journal of neurodevelopmental disorders, 8(1), 7. http://doi.org/ 10.1186/s11689016-9139-8

Karmiloff-Smith, A. (2015). An alternative to domain-general or domain-specific frameworks for theorizing about human evolution and ontogenesis. AIMS Neuroscience, 2(2), 91104. https://doi.org/10.3934/Neuroscience.2015.2.91

Leppänen, J. M., \& Nelson, C. A. (2009). Tuning the developing brain to social signals of emotions. Nature Reviews Neuroscience, 10(1), 37-47. https://doi.org/10.1038/nrn2554

Luck, S. J. (2014). An Introduction to the Event-Related Potential Technique. MIT Press.

Nakato, E., Otsuka, Y., Kanazawa, S., Yamaguchi, M., \& Kakigi, R. (2011). Distinct differences in the pattern of hemodynamic response to happy and angry facial expressions in infants-A near-infrared spectroscopic study. NeuroImage, 54(2), 1600-1606. https://doi.org/10.1016/j.neuroimage.2010.09.021

Nakato, E., Otsuka, Y., Kanazawa, S., Yamaguchi, M., Watanabe, S., \& Kakigi, R. (2009). When do infants differentiate profile face from frontal face? A near-infrared spectroscopic study. Human Brain Mapping, 30(2), 462-472. http://doi.org/10.1002/hbm.20516

Ozonoff, S., Young, G. S., Carter, A., Messinger, D., Yirmiya, N., Zwaigenbaum, L., ... \& Hutman, T. (2011). Recurrence risk for autism spectrum disorders: a Baby Siblings Research Consortium study. Pediatrics, 128(3), e488-e495.

Peltola, M.J., Leppänen, J.M., Mäki, S., Hietanen, J.K., 2009. Emergence of enhanced attention to fearful faces between 5 and 7 months of age. Social. Cogn. Affect. Neurosci. 4 (2), 134-142. http://dx.doi.org/10.1093/scan/nsn046. 
van den Boomen, C., Munsters, N., \& Kemner, C. (2019). Emotion processing in the infant brain: The importance of local information. Neuropsychologia, 126, 62-68. https://doi.org/10.1016/j.neuropsychologia.2017.09.006

van Den Boomen, C., van der Smagt, M. J., \& Kemner, C. (2012). Keep your eyes on development: the behavioral and neurophysiological development of visual mechanisms underlying form processing. Frontiers in Psychiatry, 3, 16.

Vlamings, P. H. J. M., Goffaux, V., \& Kemner, C. (2009). Is the early modulation of brain activity by fearful facial expressions primarily mediated by coarse low spatial frequency information? Journal of Vision, 9(5), 12.1-13. http://doi.org/10.1167/9.5.12

Vlamings, P. H. J. M., Jonkman, L. M., van Daalen, E., van der Gaag, R. J., \& Kemner, C. (2010). Basic abnormalities in visual processing affect face processing at an early age in autism spectrum disorder. Biological Psychiatry, 68(12), 1107-1113. http://doi.org/10.1016/j.biopsych.2010.06.024

Webb, S. J., Jones, E. J., Merkle, K., Venema, K., Greenson, J., Murias, M., \& Dawson, G. (2011). Developmental change in the ERP responses to familiar faces in toddlers with autism spectrum disorders versus typical development. Child development, 82(6), 18681886. http://doi.org/10.1111/j.1467-8624.2011.01656.x

Webb, S. J., Long, J. D., \& Nelson, C. A. (2005). A longitudinal investigation of visual eventrelated potentials in the first year of life. Developmental Science, 8(6), 605-616. https://doi.org/10.1111/j.1467-7687.2005.00452.x

White, H., Chroust, A., Heck, A., Jubran, R., Galati, A., \& Bhatt, R. S. (2019). Categorical Perception of Facial Emotions in Infancy. Infancy, 24(2), 139-161. https://doi.org/10.1111/infa.12275 

Appendix II

Acknowledgements 

I would like to gratefully acknowledge several people who were part of the four-year journey during which I worked on my thesis.

First, I would like to thank my supervisors for giving me the opportunity to undertake this research and to be part of a great European cross-disciplinary network as it is BRAINVIEW. Thanks to Chantal for providing me structure in the research process and for challenging my critical thinking. Thanks to Caroline for always being available with valuable advices and refreshing laughs, for passionate discussions, and for having faith in me. Thanks for welcoming into your home; you and your family are truly special people. Thanks to Carlijn for taking the time to have critical discussions despite being busy with other responsibilities, and for calming me during the last bits of my thesis.

Next, I want to thank all the babies and families who took part in the experiments that made this thesis possible. I believe that together we added a little piece into the 'infant face-processing' puzzle. A huge thank you to all the interns, students, and research assistants who helped me with data collection and analyses, as well as to all the colleagues, secretaries and support staff of Experimental Psychology, Kinder Kennis Centrum, and Zebra Project.

Thanks to my kamer buddies Isabell, Remo, and Febe; there is no office like ours, together we laughed, cried, screamed and jumped! Isa you are a true friend and with you nobody can get bored! Thanks for always being honest with me, for broadening my point of view, and for our dreaming chats. Remo thank you for thinking along with me about EEG and for sharing with me your Dutchness. Remo please remember that you are also a little Italian now, capito?! Febe thank you for welcoming me in the office with the kindness that characterizes you. You are one of the sweetest and most determined persons I know. Thank you for reminding me that we are in charge of our present and future. Kayla you have been a part of our office as well, thank you for all your support, countless chats and very nice dinners. Talking to you helped me a lot in these years, sometimes I felt as we run on very similar paths. Your advices have been always very useful to me. I love you guys! 
Thanks to all the PhDs and researchers of UU and of other universities for the constructive and fun moments. In particular, I would like to thank Stefanos for his friendship and support during these years. I am very glad we met at the beginning of our adventure in the Netherlands; thank you for our evening discussions on life and work. A great thank you to Chiara and Antonella, my crazy Italian buddies. Having you at UU was fantastic...una boccata di aria fresca, Frect! Thank you for all the fun and encouraging moments. Thank you to Laura, our collaboration turned into a beautiful friendship. Thanks for being always available for a chat and for all the good advices. I feel very lucky that our paths crossed in London. A huge thank you to two of my former supervisors, Prof. dr. Paola Sessa and dr. Federica Meconi. Thank you for listening to my concerns and for your support. You helped me regaining focus and purpose when I most needed it, grazie infinite! I would also like to thank Anna and Sabrina for patiently answering all my questions about NIRS and for your precious collaboration.

Thanks to all the past and present members of NeuDe lab. Thank you all for the interesting meetings and your feedbacks on my work. Thanks to all the members of BRAINVIEW as well as the funders, the European Commission's 2020 Program. Being part of such amazing group of people has been a pleasure and honour. I learned a lot from all the ESRs and PIs, and enjoyed very much our meetings across Europe. Thank you all for the wonderful experience and for being a very supportive group. I would also like to thank my committee, Prof. dr. Aoju Chen, Prof. dr. Sabine Hunnius, Prof. dr. Emily Jones, Prof. dr. Leon Kenemans, Prof. dr. Stefan van der Stigchel for taking the time to read and critically evaluate my dissertation.

Thanks to my paranymphs, Rianne and Atanaska. Rianne thanks for your tremendous support and true friendship. I feel very lucky that I met you, I could not do this without you. Thanks for sharing with me the ups and downs of our PhDs, for our great time in UK, and for our cheerful time with meneer Konijn. You had my back, always! Thanks to Atanaska, the housemate everyone would want! Assya thank you for being a good friend, for your help with Dutch stuff, and for our evening chats. Thanks to you, our house feels like home to me. 
Thanks to all my international friends for being so supportive and for all our fun memories (the old ones and the ones that still need to come!). Sonia, you have been a true friend since the first year of our bachelor program in Padova. Thank you for our long catch-up chats and for our countless laughs! Thank you to my ERASMUS family, Francesco, Iro, Maria C., Maria O., Magi, George, Carlos, Nikolas, Tim, Tom, for our reunions and chats, you are all beautiful souls! Thanks to Stefania, for being a good friend in our tiny town, and to Claudia as well for our catch-up drinks! I always look forward to these moments when I come back to Tione. Thanks to Diana for our overseas Skype sessions and great laughs; thank you for all the support and for your friendship. Thanks to my Dutch dancers friends, Eline and Wieke. Our dinners and lindy hop adventures have been refreshing in these years. Thank you for all the good moments. Thanks to Anna and Madeleine for listening to my frustrations and for celebrating with me my successes.

A special thank you to my family who tried to keep me healthy and sane in these years, despite the distance. Thanks to Mamma, Papà and my brother Leo for supporting me all these years and believing in me. Thanks to my Nonna, saggia e amorevole, grazie per avermi rassicurato in questi anni e per volermi un sacco di bene; ti voglio un mondo di bene. Thanks to all my zii, in particular to zia Mara, zio Angelo, and zio Nello, even if you do not realize it you were of great support in these years. Thank you, my dear family!

Finally, I want to thank Stefano, my love, my best friend. Thank you for being always on my side, for being patient and for believing in me even when I didn't. Your support has been vital in these years. Thanks for finding a way to be in the Netherlands and for our great trips around the world; these moments gave me the energy to complete this thesis.

Thank you all! 

Appendix III

List of Publications 



\section{Publications in this dissertation}

Di Lorenzo, R., Blasi, A., Junge, C., van den Boomen, C., van Rooijen, R., \& Kemner, C. (2019). Brain responses to faces and facial expressions in 5-month-olds: an fNIRS study. Frontiers in psychology, 10, 1240. https://doi.org/10.3389/fpsyg.2019.01240

Di Lorenzo, R., Munsters, N., Ward., E. K., de Jonge, M., Kemner, C., \& van den Boomen, C. (Under revision). Is it fear? Similar brain responses to fearful and neutral faces in infants with a heightened likelihood for Autism Spectrum Disorder

Di Lorenzo, R., Pirazzoli, L., Blasi, A., Bulgarelli, C., Hakuno, Y., Minagawa, Y., \& Brigadoi, S. (2019). Recommendations for motion correction of infant fNIRS data applicable to multiple data sets and acquisition systems. NeuroImage, 200, 511 527. http://doi.org/10.1016/j.neuroimage.2019.06.056

Di Lorenzo, R., van den Boomen, C., Kemner, C., \& Junge, C. (Under revision). Searching for robust infant electrophysiological markers of face-sensitivity: results from a large longitudinal sample of infants.

\section{Publications not in this dissertation}

Cribb, S. J., Olaithe, M., Di Lorenzo, R., Dunlop, P. D., \& Maybery, M. T. (2016). Embedded figures test performance in the broader autism phenotype: a metaanalysis. Journal of Autism and Developmental Disorders, 46(9), 2924-2939. https://doi.org/10.1007/s10803-016-2832-3

Tan, D. W., Maybery, M. T., Clarke, M. W., Di Lorenzo, R., Evans, M. O., Mancinone, M., ... \& Whitehouse, A. J. (2018). No relationship between autistic traits and salivary testosterone concentrations in men from the general population. PloS one, 13(6), e0198779. https://doi.org/10.1371/journal.pone.0198779

Tan, D. W., Maybery, M. T., Evans, M. O., Di Lorenzo, R., \& Whitehouse, A. J. (2017). Sex-specific variations in perceived facial masculinity/femininity across levels of autistic-like traits in the general population. The face predicts the brain: An investigation of facial morphology associated with autism, 63. 

Appendix IV

About the Author 



\begin{abstract}
About the Author

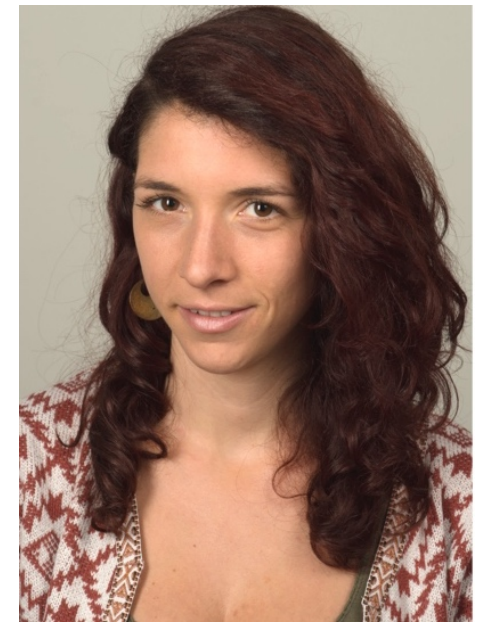

Renata Di Lorenzo was born in Tione di Trento, Italy, on the $6^{\text {th }}$ of January 1988. In 2007 she graduated from high school and decided to start a bachelor in Psychological Cognitive and Psychobiological Sciences at the University of Padua, Italy. She graduated in 2010. Next, she decided to continue her studies in Padua and in 2011 she enrolled in the master of Neuroscience and Neuropsychological Rehabilitation. In 2012, Renata won the Erasmus scholarship which allowed her to spend seven fulfilling months at Linkoping University, Sweden. After this period, she went back to Padua and completed her master degree in 2013 cum laude. In the following year, she explored different post-graduate carriers. First, she spent six months at the University Hospital of Padua as intern of the Paediatric Department of Neuropsychology, where she learned how to conduct clinical/cognitive assessments with children affected by epilepsy. Next, she moved to Perth, Australia, to complete a six-months internship at the University of Western Australia. In Perth, she was involved in research that focused on the study of Autism Spectrum Disorder. After this period, she decided to continue research in this field and applied for a relevant $\mathrm{PhD}$ position at Utrecht University. In 2015, she moved to the Netherlands and started her $\mathrm{PhD}$ as part of the Marie-Sklodowska-Curie International Training Network, Brainview. As PhD candidate, she investigated the typical and atypical development of face processing in infancy. Currently, she is evaluating different career options.
\end{abstract}


